\title{
3-D Axisymmetric Transonic Shock Solutions of the Full Euler System in Divergent Nozzles
}

\author{
YONG PARK(1)
}

Communicated by N. MASMOUDI

\begin{abstract}
We establish the stability of 3-D axisymmetric transonic shock solutions of the steady full Euler system in divergent nozzles under small perturbations of an incoming radial supersonic flow and a constant pressure at the exit of the nozzles. To study 3-D axisymmetric transonic shock solutions of the full Euler system, we use a stream function formulation of the full Euler system for a 3-D axisymmetric flow. We resolve the singularity issue arising in stream function formulations of the full Euler system for a 3-D axisymmetric flow. We develop a new scheme to determine a shock location of a transonic shock solution of the steady full Euler system based on the stream function formulation.
\end{abstract}

\section{Introduction}

In [14, Chapter 147], the authors, using an approximate model, described a transonic shock phenomenon for a compressible invicid flow of an ideal polytropic gas in a convergent-divergent type nozzle called de Laval nozzle: if a subsonic flow accelerating as it passes through the convergent part of the nozzle reaches the sonic speed at the throat of the nozzle, then it becomes a supersonic flow right after the throat of the nozzle. It further accelerates as it passes through the divergent part of the nozzle. If an appropriately large exit pressure $p_{e}$ is imposed at the exit of the nozzle, then at a certain place of the divergent part of the nozzle, a shock front intervenes, the flow is compressed and slowed down to subsonic speed. The position and strength of the shock front are automatically adjusted so that the end pressure at the exit becomes $p_{e}$. This phenomenon was rigorously studied using radial solutions of the full Euler system in [31] (it was shown that in a divergent nozzle, for a given constant supersonic data on the entrance of the nozzle and appropriately large constant pressure on the exit of the nozzle, there exists a unique radial transonic shock solution satisfying these conditions). Motivated by this phenomenon, there 
were many studies on the stability of transonic shock solutions in divergent nozzles (structural stability of radial transonic shock solutions in divergent nozzles under multi-dimensional perturbations of an entrance supersonic data and exit pressure) and related problems.

The stability of one-dimensional transonic shock solutions in flat nozzles was studied first. This subject was studied using the potential flow model in [7-9,26,27] and further studied using the full Euler system in [5,6,11-13,25,29,30]. These results showed that one-dimensional transonic shock solutions in flat nozzles are not stable under a perturbation of a physical boundary condition (supersonic data on the entrance or density, pressure or normal velocity on the exit) and, even if onedimensional transonic shock solutions in flat nozzles are stable, their shock locations are not uniquely determined unless there exists the assumption that a shock location passes through some point on the wall of the nozzle, as it can be expected from the behavior of one-dimensional transonic shock solutions in flat nozzles (as a shock location changes, the value of the subsonic part of an one-dimensional transonic shock solution in a flat nozzle does not change). After that, the stability of radial transonic shock solutions in divergent nozzles was studied. This subject was first studied using the full Euler system in $[19,28]$. In these results, the authors, by considering a perturbation of radial transonic shock solutions in divergent nozzles, could show that a shock location is uniquely determined for a given exit pressure without the assumption that a shock location passes through some point of the nozzle but they only had the result under the assumption that the tip angle of the nozzle is sufficiently small. The stability of radial transonic shock solutions in divergent nozzle without restriction on the tip angle of the nozzle was studied after that. In [3], the authors studied this subject using the non-isentropic potential model introduced in [3], and they obtained the stability result for radial transonic shock solutions in divergent nozzles. This subject was also studied using the full Euler system. For the 2-D case this was done in $[18,21,24]$. In these papers, the authors had the stability result for radial transonic shock solutions in divergent nozzles. In particular, the authors in [21] had the result for flows having $C^{1, \alpha}$ interior and $C^{\alpha}$ up to boundary regularity, so that they could consider a general perturbation of a nozzle. This for the 3-D case for axisymmetric flows with zero angular momentum components was done in [20]. The authors in this paper also had the same result. This, for the general 3-D case, was done in [10,23]. The authors in [10,23] also had the same result but under S-condition introduced in [10,23]. Recently, this subject for the general 3-D case for flows having some friction term was studied in [32].

In this paper, we study the stability of radial transonic shock solutions in divergent nozzles under small perturbations of an incoming radial supersonic solution and a constant exit pressure using the full Euler system for the 3-D case for axisymmetric flows. We consider axisymmetric flows with non-zero angular momentum components. (This is a difference from [20].) We consider a divergent nozzle having no restriction on the tip angle of the nozzle and do not have any assumption on an incoming supersonic solution.

The main new feature in this paper is to develop a new iteration scheme to determine a shock location for a transonic shock solution of the steady full Euler 
system in a divergent nozzle and resolve the singularity issue arising in the stream function formulations of the full Euler system using an elliptic system approach.

To deal with the stability of 3-D axisymmetric transonic shock solutions of the full Euler system, we use a stream function formulation for the full Euler system for an axisymmetric flow. This formulation shows the fact that an initial shock position and a shape of a shock location (see the definitions below the proof of Theorem 2) are determined in different mechanisms clearly. Based on this formulation and using the fact that the entropy of the downstream subsonic solution of a radial transonic shock solution on a shock location monotonically increases as a shock location moves toward the exit of the nozzle (see Lemma 2), we develop a new scheme to determine a shock location of a transonic shock solution of the full Euler system in a divergent nozzle: 1. Pseudo Free Boundary Problem 2. Determination of a shape of a shock location (see below the proof of Theorem 2).

In technical part, we resolve the singularity issue arising in stream function formulations of the full Euler system. A stream function formulation when it is formulated by using the Stokes' stream function (see (2.23)) has a singularity issue at the axis of symmetry. We resolve this singularity issue by formulating a stream function formulation using the vector potential form of the stream function (see $\S 2.4$ ) and solving a singular elliptic equation appearing in this stream function formulation as an elliptic system (see $\S 3.2$ ). The stream function formulation formulated by using the vector potential form of the stream function still contains a singular elliptic equation. We transform this singular elliptic equation into a form of an elliptic system, and solve the elliptic system form as an elliptic system to solve the singular elliptic equation. (We also use this approach to prove the orthogonal completeness of eigenfunction of an associated Legendre problem of type $m=1$ with a general domain (see Lemma 18).) Using the stream function formulation formulated by using the vector potential form of the stream function, we obtain the stability result for flows having $C^{1, \alpha}$ interior and $C^{\alpha}$ up to boundary regularity.

This paper is organized as follows: in Section 2, we present definitions and a basic lemma used throughout this paper and introduce our problem and result. In this section, we introduce the stream function formulation used in this paper. In Section 3, we solve the Pseudo Free Boundary Problem. In this section, we study a linear boundary value problem for a singular elliptic equation and an initial value problem of a transport equation appearing in the Pseudo Free Boundary Problem, and prove the unique existence of solutions of the Pseudo Free Boundary Problem. In Section 4, we show the existence and uniqueness of transonic shock solutions. In Section 5, we present some computations done by using the tensor notation given in $\S 3.2$, and make up some fact needed for the well-definedness of the stream function defined in (2.23). 


\section{Problem and Theorem}

\subsection{Preliminaries}

In this paper, we consider a 3-D steady compressible invicid flow of an ideal polytropic gas. The motion of this flow is governed by the full Euler system

$$
\left\{\begin{array}{l}
\operatorname{div}(\rho \mathbf{u})=0, \\
\operatorname{div}(\rho \mathbf{u} \otimes \mathbf{u}+p \mathbb{I})=0, \\
\operatorname{div}(\rho \mathbf{u} B)=0,
\end{array}\right.
$$

where $\rho, \mathbf{u}$ and $p$ are the density, velocity and pressure of the flow, $\mathbb{I}$ is the $3 \times 3$ identity matrix and $B$ is the Bernoulli invariant of the flow given by

$$
B=\frac{|\mathbf{u}|^{2}}{2}+\frac{\gamma p}{(\gamma-1) \rho}
$$

for a constant $\gamma>1$. Types of the flow are classified by the quantity $M:=\frac{|\mathbf{u}|}{c}$ called Mach number where $c$ is the sound speed of the flow given by

$$
c:=\sqrt{\frac{\gamma p}{\rho}}
$$

for an ideal polytropic gas; if $M>1$, then a flow is called supersonic, if $M=1$, then it is called sonic and if $M<1$, then it is called subsonic. It is generally known that types of the system vary depending on the value of $M$. If $M>1$, then the system is a hyperbolic system and if $M<1$, then the system is an elliptic-hyperbolic coupled system.

When the flow passes through a domain having a certain geometric structure or satisfies a certain boundary condition, it may have a discontinuity across a surface in the domain in the direction of the flow. In this case, the surface is called a shock.

A shock solution of (2.1) is a solution of (2.1) having a shock in a domain, and is defined as follows:

Definition 1. (Shock solution) Let $\Omega$ be an open connected set in $\mathbb{R}^{3}$. Assume that a $C^{1}$ surface $\Gamma$ in $\Omega$ divides $\Omega$ into two nonempty disjoint subset $\Omega^{ \pm}$such that $\Omega=\Omega^{+} \cup \Gamma \cup \Omega^{-}$. Then a solution $(\rho, \mathbf{u}, p)$ of $(2.1)$ is called a shock solution of (2.1) with a shock $\Gamma$ if

(i) $(\rho, \mathbf{u}, p)$ is in $\left(C^{0}\left(\overline{\Omega^{ \pm}}\right) \cap C^{1}\left(\Omega^{ \pm}\right)\right)^{3}$,

(ii) $\left.(\rho, \mathbf{u}, p)\right|_{\overline{\Omega^{-}} \cap \Gamma} \neq\left.(\rho, \mathbf{u}, p)\right|_{\overline{\Omega^{+}} \cap \Gamma}$,

(iii) $\left.\rho \mathbf{u}\right|_{\overline{\Omega^{-}}} \cdot \boldsymbol{v} \neq 0$ on $\Gamma$,

(iv) and $(\rho, \mathbf{u}, p)$ satisfies (2.1) pointwisely in $\Omega^{ \pm}$and the Rankine-Hugoniot conditions

$$
[\rho \mathbf{u} \cdot \boldsymbol{v}]_{\Gamma}=\left[\mathbf{u} \cdot \boldsymbol{\tau}_{1}\right]_{\Gamma}=\left[\mathbf{u} \cdot \boldsymbol{\tau}_{2}\right]_{\Gamma}=\left[\rho(\mathbf{u} \cdot \boldsymbol{v})^{2}+p\right]_{\Gamma}=[B]_{\Gamma}=0,
$$

where $\boldsymbol{v}$ is the unit normal vector field on $\Gamma$ pointing toward $\Omega^{+}$and $\boldsymbol{\tau}_{i}$ for $i=1,2$ are unit tangent vector fields on $\Gamma$ perpendicular to each other at each point on $\Gamma$ and

$$
[F]_{\Gamma}:=\left.F\right|_{\Omega^{-}}(x)-\left.F\right|_{\overline{\Omega^{+}}}(x) \text { for } x \in \Gamma .
$$


It is said that a shock solution is physically admissible if it satisfies the following entropy condition:

Definition 2. (Entropy condition) Let $(\rho, \mathbf{u}, p)$ be a shock solution of (2.1) defined in Definition 1. Without loss of generality, assume that $\mathbf{u} \mid \overline{\Omega^{-}} \cdot \boldsymbol{v}>0$ on $\Gamma$. Then $\left.S\right|_{\overline{\Omega^{+}} \cap \Gamma}>\left.S\right|_{\overline{\Omega^{-}} \cap \Gamma}$ where $S:=\frac{p}{\rho^{\gamma}}$ is called the entropy condition.

Definition 3. In this paper, we call $S$ the entropy of $(\rho, \mathbf{u}, p)$.

Using the definition of a shock solution, a transonic shock solution of (2.1) is defined as follows:

Definition 4. A shock solution of (2.1) in Definition 1 is called a transonic shock solution if it satisfies $M>1$ in $\overline{\Omega^{-}}$and $M<1$ in $\overline{\Omega^{+}}$or the otherway around.

Remark 1. If a transonic shock solution of (2.1) satisfies $\mathbf{u} \mid \overline{\Omega^{-}} \cdot \boldsymbol{v}>0$ on $\Gamma, M>1$ in $\overline{\Omega^{-}}$and $M<1$ in $\overline{\Omega^{+}}$, then it satisfies the entropy condition.

In this paper, we deal with a 3-D axisymmetric transonic shock solution of (2.1). For precise statement, we define an axisymmetric domain and axisymmetric functions used in this paper. For later use, we present a lemma that can be used to deal with regularities of axisymmetric functions.

In this paper, we use the spherical coordinate system $(r, \theta, \varphi)$ given by the relation

$$
(x, y, z)=(r \sin \theta \cos \varphi, r \sin \theta \sin \varphi, r \cos \theta),
$$

where $(x, y, z)$ is the Cartesian coordinate system in $\mathbb{R}^{3}$. The unit vectors in this coordinate system are given by

$$
\begin{aligned}
& \mathbf{e}_{r}=\sin \theta \cos \varphi \mathbf{e}_{1}+\sin \theta \sin \varphi \mathbf{e}_{2}+\cos \theta \mathbf{e}_{3}, \\
& \mathbf{e}_{\theta}=\cos \theta \cos \varphi \mathbf{e}_{1}+\cos \theta \sin \varphi \mathbf{e}_{2}-\sin \theta \mathbf{e}_{3}, \\
& \mathbf{e}_{\varphi}=-\sin \varphi \mathbf{e}_{1}+\cos \varphi \mathbf{e}_{2},
\end{aligned}
$$

where $\mathbf{e}_{i}$ for $i=1,2,3$ are the unit vector in the $x, y$ and $z$ direction, respectively.

Using this spherical coordinate system, an axisymmetric domain and axisymmetric functions are defined as follows:

Definition 5. Let $\Omega \subset \mathbb{R}^{3}$. $\Omega$ is called axisymmetric if $(x, y, z) \in \Omega$, then

$$
\left(\sqrt{x^{2}+y^{2}} \cos \varphi, \sqrt{x^{2}+y^{2}} \sin \varphi, z\right) \in \Omega
$$

for $\varphi \in[0,2 \pi)$. A function $f: \Omega \rightarrow \mathbb{R}$ is called axisymmetric if $f$ is independent of $\varphi$ as a function of the spherical coordinate system. A vector valued function $\mathbf{u}: \Omega \rightarrow \mathbb{R}^{3}$ is called axisymmetric if $u_{r}=\mathbf{u} \cdot \mathbf{e}_{r}, u_{\theta}=\mathbf{u} \cdot \mathbf{e}_{\theta}$ and $u_{\varphi}=\mathbf{u} \cdot \mathbf{e}_{\varphi}$ are axisymmetric.

Definition 6. In this paper, when a velocity field $\mathbf{u}$ is represented as $\mathbf{u}=u_{r} \mathbf{e}_{r}+$ $u_{\theta} \mathbf{e}_{\theta}+u_{\varphi} \mathbf{e}_{\varphi}, u_{\varphi} \mathbf{e}_{\varphi}$ is called the angular momentum component of $\mathbf{u}$. 
For later use, we present the following lemma that shows when an axisymmetric function in $C^{k}$ as a function of the spherical coordinate system is in $C^{k}$ as a function of the Cartesian coordinate system (this lemma is obtained from [22, Corollary 1]):

Lemma 1. Let $\Omega$ be an axisymmetric connected open set in $\mathbb{R}^{3}$ that does not contain the origin. Suppose that a function $f: \Omega \rightarrow \mathbb{R}$ is axisymmetric. Then

(i) $f$ and $f \mathbf{e}_{r}$ are in $C^{k}(\Omega)$ for $k \in 0,1,2, \ldots$ if and only if $f$ is in $C^{k}$ as a function of spherical coordinate system in $\Omega$ and $\partial_{\theta}^{2 m+1} f=0$ for all $0 \leq m \leq\left\lfloor\frac{k-1}{2}\right\rfloor$.

(ii) $f \mathbf{e}_{\theta}$ and $f \mathbf{e}_{\varphi}$ are in $C^{k}(\Omega)$ for $k \in 0,1,2, \ldots$ if and only if $f$ is in $C^{k}$ as a function of spherical coordinate system in $\Omega$ and $\partial_{\theta}^{2 m} f=0$ for all $0 \leq m \leq\left\lfloor\frac{k}{2}\right\rfloor$.

In this paper, we use the same function notation when we represent an axisymmetric function as a function on the Cartesian coordinate system or spherical coordinate system.

\subsection{Radial transonic shock solution}

Let $r_{0}, r_{1}$ and $\theta_{1}$ be constants such that $0<r_{0}<r_{1}$ and $0 \leq \theta_{1}<\pi$. Define a divergent nozzle by

$$
\mathcal{N}:=\left\{(x, y, z) \in \mathbb{R}^{3} \mid r_{0}<r<r_{1}, 0 \leq \theta<\theta_{1}\right\} .
$$

To introduce our problem and for our later analysis, we study a radial transonic shock solution of (2.1) in $\mathcal{N}$.

Fix positive constants $\left(\rho_{i n}, u_{i n}, p_{i n}\right)$ satisfying $M_{i n}\left(:=u_{i n} / \sqrt{\frac{\gamma p_{i n}}{\rho_{i n}}}\right)>1$. Let $\left(\bar{\rho}, \bar{u} \mathbf{e}_{r}, \bar{p}\right)$ be a radial shock solution of (2.1) in $\mathcal{N}$ with a shock $\Gamma_{t}:=\{r=t\} \cap \mathcal{N}$ for some $t \in\left[r_{0}, r_{1}\right]$ satisfying

$$
\left(\bar{\rho}, \bar{u} \mathbf{e}_{r}, \bar{p}\right)=\left(\rho_{i n}, u_{i n} \mathbf{e}_{r}, p_{i n}\right) \quad \text { on } \quad \Gamma_{e n}:=\partial \mathcal{N} \cap\left\{r=r_{0}, 0 \leq \theta<\theta_{1}\right\} .
$$

Then, by (2.1) and (2.3), ( $\bar{\rho}, \bar{u}, \bar{p})$ is a solution of

$$
\left\{\begin{array}{l}
\left(r^{2} \bar{\rho} \bar{u}\right)^{\prime}=0 \\
\bar{\rho} \bar{u} \bar{u}^{\prime}+\bar{p}^{\prime}=0 \\
\bar{\rho} \bar{u} \bar{B}^{\prime}=0
\end{array}\right.
$$

with

$$
(\bar{\rho}, \bar{u}, \bar{p})\left(r_{0}\right)=\left(\rho_{i n}, u_{i n}, p_{i n}\right)
$$

in $\overline{D_{t}^{-}}$, where $D_{t}^{-}:=\left\{r_{0}<r<t\right\}, \bar{B}:=\frac{\bar{u}^{2}}{2}+\frac{\gamma \bar{p}}{(\gamma-1) \bar{\rho}}$ and 'is the derivative with respect to $r$, and is a solution of (2.6) with

$$
\left\{\begin{array}{l}
\bar{\rho} \bar{u}(t)=\left.\bar{\rho} \bar{u}\right|_{\overline{D_{t}^{-}}}(t), \\
\left(\bar{\rho} \bar{u}^{2}+\bar{p}\right)(t)=\left.\left(\bar{\rho} \bar{u}^{2}+\bar{p}\right)\right|_{\overline{D_{t}^{-}}}(t), \\
\bar{B}(t)=\left.\bar{B}\right|_{\overline{D_{t}^{-}}}(t)
\end{array}\right.
$$


in $\overline{D_{t}^{+}}$where $D_{t}^{+}:=\left\{t<r<r_{1}\right\}$.

From (2.6), one can obtain

$$
\bar{S}^{\prime}=0,
$$

where $\bar{S}:=\frac{\bar{p}}{\bar{\rho}^{\gamma}}$. Using this equation, one can see that a solution $(\bar{\rho}, \bar{u}, \bar{p})$ of (2.6) with (2.7) satisfies

$$
\left\{\begin{array}{l}
r^{2} \bar{\rho} \bar{u}=m_{0}, \\
\bar{S}=S_{i n}, \\
\bar{B}=B_{0},
\end{array}\right.
$$

where $m_{0}:=r_{0}^{2} \rho_{i n} u_{i n}, S_{i n}:=\frac{p_{i n}}{\rho_{i n}^{\gamma}}$ and $B_{0}:=\frac{u_{i n}^{2}}{2}+\frac{\gamma p_{i n}}{(\gamma-1) \rho_{i n}}$ on the domain where (2.6) with (2.7) has a unique solution $(\bar{\rho}, \bar{u}, \bar{p})$. Using this fact, the local unique existence theorem for ODE and the condition that $M_{i n}>1$, it can be shown that (2.6) with (2.7) has a unique solution $(\bar{\rho}, \bar{u}, \bar{p})$ satisfying $\bar{M}>1$ and $\bar{M}^{\prime}>0$ in $\left[r_{0}, r_{1}\right]$ where $\bar{M}:=\bar{u} / \sqrt{\frac{\gamma \bar{p}}{\bar{\rho}}}$. From this, we have that (2.8) is well-defined for any $t \in\left[r_{0}, r_{1}\right]$.

From (2.8), we can obtain

$$
\bar{u}(t)=\left.\frac{\bar{K}}{\bar{u}}\right|_{D_{t}^{-}}(t),
$$

where $\bar{K}:=\frac{2(\gamma-1)}{\gamma+1} \bar{B}$, and

$$
\bar{S}(t)=\left(g\left(\bar{M}^{2}\right) \bar{S}\right) \mid \overline{D_{t}^{-}}(t),
$$

where

$$
g(x):=\frac{1}{\gamma+1}(2 \gamma x-(\gamma-1))\left(\frac{\gamma-1}{\gamma+1}+\frac{2}{\gamma+1} \frac{1}{x}\right)^{\gamma} .
$$

Using (2.11), the third equations of (2.8) and (2.10) and $\bar{M} \mid \overline{D_{t}^{-}}(t)>1$, it can be checked that $(\bar{\rho}, \bar{u}, \bar{p})$ satisfying (2.8) satisfies $\bar{M}(t)<1$. By (2.9), (2.10) and (2.12), a solution $(\bar{\rho}, \bar{u}, \bar{p})$ of (2.6) with (2.8) satisfies

$$
\left\{\begin{array}{l}
r^{2} \bar{\rho} \bar{u}=m_{0}, \\
\bar{S}=g\left(\bar{M} \mid \frac{2}{D_{t}^{-}}(t)\right) S_{i n} \\
\bar{B}=B_{0}
\end{array}\right.
$$

on the domain where (2.6) with (2.8) has a unique solution $(\bar{\rho}, \bar{u}, \bar{p})$. Using these two facts and the local unique existence theorem for ODE, it can be shown that (2.6) with (2.8) has a solution $(\bar{\rho}, \bar{u}, \bar{p})$ satisfying $\bar{M}<1$ and $\bar{M}^{\prime}<0$ in $\overline{D_{t}^{+}}$. Combining this fact with the fact that (2.6) with (2.7) has a unique solution $(\bar{\rho}, \bar{u}, \bar{p})$ satisfying $\bar{M}>1$ in $\left[r_{0}, r_{1}\right]$, we have that a radial shock solution $\left(\bar{\rho}, \bar{u} \mathbf{e}_{r}, \bar{p}\right)$ uniquely exists in $\mathcal{N}$ for each $t \in\left[r_{0}, r_{1}\right]$ and is a radial transonic shock solution. 
Remark 2. By (2.6) and the fact that a solution $(\bar{\rho}, \bar{u}, \bar{p})$ of (2.6) with (2.8) uniquely exists in $\overline{D_{t}^{+}}$satisfying (2.14), we have that a solution $(\bar{\rho}, \bar{u}, \bar{p})$ of (2.6) with (2.8) satisfies

$$
\bar{\rho}^{\prime}=\frac{2 \bar{\rho}}{r} \frac{\bar{M}^{2}}{1-\bar{M}^{2}} \text { in } D_{t}^{+}
$$

From this fact and the fact that $\bar{M} \mid \overline{D_{t}^{+}}<1$ in $\overline{D_{t}^{+}}$, we obtain $\bar{\rho} \mid \frac{1}{D_{t}^{+}}>0$ in $\overline{D_{t}^{+}}$.

One can see that the values of $\left.(\bar{\rho}, \bar{u}, \bar{p})\right|_{D_{t}^{+}}$at a fixed location $r$ in $\overline{D_{t}^{+}}$are determined by the three conserved quantities on the right hand sides of the equations in (2.14). This combined with the fact that the conserved quantity for $\bar{S}$ in $\overline{D_{t}^{+}}$given in (2.14) varies depending on $t$ (obtained from $(2.14)$ by using $\left(\bar{M} \mid \overline{D_{t}^{-}}\right)^{\prime}>0$ in $\overline{D_{t}^{-}}$for any $\left.t \in\left[r_{0}, r_{1}\right]\right)$ implies that the values of $\left.(\bar{\rho}, \bar{u}, \bar{p})\right|_{\overline{D_{t}^{+}}}$at a fixed location $r$ in $\overline{D_{t}^{+}}$ vary depending on $t$. To represent this dependence, we write $\left.(\bar{\rho}, \bar{u}, \bar{p})\right|_{\overline{D_{t}^{+}}}(r)$ and $\bar{S} \mid \frac{}{D_{t}^{+}}(r)$ as $\left.(\bar{\rho}, \bar{u}, \bar{p})\right|_{D_{t}^{+}}(r ; t)$ and $\bar{S} \mid \frac{}{D_{t}^{+}}(r ; t)$, respectively.

By direct computation, one can see that the conserved quantity for $\bar{S}$ in $\overline{D_{t}^{+}}$ satisfies some monotonicity with respect to $t$. This is given in the following lemma:

Lemma 2. Let $r_{0}, r_{1}, t$ be positive constants such that $r_{0} \leq t \leq r_{1}$. Suppose that $(\bar{\rho}, \bar{u}, \bar{p})$ is as above. Then it holds that

$$
\frac{d \bar{S} \mid \frac{}{D_{t}^{+}}(t ; t)}{d t}>0 \text { for any } t \in\left[r_{0}, r_{1}\right]
$$

Proof. Differentiate $\left.\bar{S}\right|_{D_{t}^{+}}(t ; t)$ with respect to $t$. Then we have

$$
\frac{\left.d \bar{S}\right|_{\overline{D_{t}^{+}}}(t ; t)}{\mathrm{d} t}=\left.\frac{d g(x)}{d x}\right|_{x=\left(\bar{M} \mid \overline{D_{t}^{-}}\right)^{2}(t)} \frac{d\left(\bar{M} \mid \overline{D_{t}^{-}}\right)^{2}(t)}{\mathrm{d} t} S_{i n} .
$$

One can easily check that $g(1)=1$ and $g^{\prime}(x)>0$ for all $x>1$. By this fact, $\bar{M} \mid \overline{D_{t}^{-}}(t)>1$ and $\left(\bar{M} \mid \overline{D_{t}^{-}}\right)^{\prime}(t)>0$ for any $t \in\left[r_{0}, r_{1}\right]$, we obtain from (2.15) the desired result.

From Lemma 2, we can obtain

Proposition 1. Suppose that $r_{0}, r_{1}, t$ and $(\bar{\rho}, \bar{u}, \bar{p})$ are as in Lemma 2. Then, for any $t \in\left[r_{0}, r_{1}\right]$,

$$
\frac{d \bar{p} \mid \overline{D_{t}^{+}}}{d t}\left(r_{1} ; t\right)<0 .
$$


Proof. By the definitions of $\bar{B}$ and $\bar{S}$ and the first and third equation of (2.14),

$$
B_{0}=\left.\left(\frac{1}{2}\left(\frac{m_{0}}{r_{1}^{2}}\right)^{2}\left(\frac{\bar{S}}{\bar{p}}\right)^{\frac{2}{\gamma}}+\frac{\gamma}{\gamma-1} \bar{p}^{1-\frac{1}{\gamma}} \bar{S}^{\frac{1}{\gamma}}\right)\right|_{\overline{D_{t}^{+}}}\left(r_{1} ; t\right) .
$$

Differentiate this with respect to $t$. Then we get

$$
\frac{d \bar{p} \mid \frac{}{D_{t}^{+}}\left(r_{1} ; t\right)}{\mathrm{d} t}=-\left.\left(\frac{\left(\frac{m_{0}}{r_{1}^{2}}\right)^{2}\left(\frac{\bar{S}}{\bar{p}}\right)^{\frac{2}{\gamma}-1}+\frac{\gamma}{\gamma-1} \bar{p}^{2-\frac{1}{\gamma}} \bar{S}^{\frac{1}{\gamma}-1}}{-\left(\frac{m_{0}}{r_{1}^{2}}\right)^{2}\left(\frac{\bar{S}}{\bar{p}}\right)^{\frac{2}{\gamma}}+\gamma \bar{p}^{1-\frac{1}{\gamma}} \bar{S}^{\frac{1}{\gamma}}}\right)\right|_{\frac{d \bar{S} \mid}{D_{t}^{+}}}\left(r_{1} ; t\right) \frac{\mathrm{d} t}{\mathrm{~d} t}\left(r_{1} ; t\right)
$$

By $\left.\bar{M}\right|_{D_{t}^{+}}<1$ in $\overline{D_{t}^{+}}$, Lemma 2 and the second equation of (2.14), we obtain from (2.16) the desired result.

The above proposition implies that for any given $p_{c} \in\left[p_{1}, p_{2}\right]$ where $p_{1}:=$ $p_{0} \mid \overline{D_{r_{1}}^{+}}\left(r_{1} ; r_{1}\right)$ and $p_{2}:=p_{0} \mid \overline{D_{r_{0}}^{+}}\left(r_{1} ; r_{0}\right)$, there is a unique shock location $\Gamma_{t}$ in $\overline{\mathcal{N}}$ such that $\left(\bar{\rho}, \bar{u} \mathbf{e}_{r}, \bar{p}\right)$ satisfies $\left.\bar{p}\right|_{D_{t}^{+}}\left(r_{1} ; t\right)=p_{c}$. Hereafter, we fix a constant $p_{c} \in\left(p_{1}, p_{2}\right)$ and denote $t \in\left(r_{0}, r_{1}\right)$ such that a radial transonic shock solution of (2.1) satisfying (2.5) and having a shock location $\Gamma_{t}$ satisfies $p\left(r_{1}\right)=p_{c}$ by $r_{s}$. Also, we denote a solution $(\rho, u, p)$ of (2.6) with (2.7) and a solution $(\rho, u, p)$ of (2.6) with (2.8) for $t=r_{s}$ by $\left(\rho_{0}^{-}, u_{0}^{-}, p_{0}^{-}\right)$and $\left(\rho_{0}^{+}, u_{0}^{+}, p_{0}^{+}\right)$, respectively, and denote $\frac{p_{0}^{+}}{\rho_{0}^{+\gamma}}$ by $S_{0}^{+}$. By the local unique existence theorem for ODE, there exists a positive constant $\delta_{1}$ such that $\left(\rho_{0}^{+}, u_{0}^{+}, p_{0}^{+}\right)$uniquely exists in $\left[r_{s}-\delta_{1}, r_{s}\right]$ satisfying $M_{0}^{+}\left(=u_{0}^{+} / \sqrt{\frac{\gamma p_{0}^{+}}{\rho_{0}^{+}}}\right)<1$. Let $\delta_{1}$ be one such constant.

\subsection{Problem}

Using the radial transonic shock solution given in the previous subsection, we present our problem.

In this paper, we use the following weighted Hölder norm. For a bounded connected open set $\Omega \subset \mathbb{R}^{n}$, let $\Gamma$ be a closed portion of $\partial \Omega$. For x, y $\in \Omega$, set

$$
\delta_{\mathrm{x}}:=\operatorname{dist}(\mathrm{x}, \Gamma) \text { and } \delta_{\mathrm{x}, \mathrm{y}}:=\min \left(\delta_{\mathrm{x}}, \delta_{\mathrm{y}}\right) .
$$

For $k \in \mathbb{R}, \alpha \in(0,1)$ and $m \in \mathbb{Z}^{+}$, we define

$$
\begin{aligned}
\|u\|_{m, 0, \Omega}^{(k, \Gamma)} & :=\sum_{0 \leq|\beta| \leq m} \sup _{\mathrm{x} \in \Omega} \delta_{\mathrm{x}}^{\max (|\beta|+k, 0)}\left|D^{\beta} u(\mathrm{x})\right| \\
{[u]_{m, \alpha, \Omega}^{(k, \Gamma)} } & :=\sum_{|\beta|=m} \sup _{\mathrm{x}, \mathrm{y} \in \Omega, \mathrm{x} \neq \mathrm{y}} \delta_{\mathrm{x}, \mathrm{y}}^{\max (m+\alpha+k, 0)} \frac{\left|D^{\beta} u(\mathrm{x})-D^{\beta} u(\mathrm{y})\right|}{|\mathrm{x}-\mathrm{y}|^{\alpha}} \\
\|u\|_{m, \alpha, \Omega}^{(k, \Gamma)} & :=\|u\|_{m, 0, \Omega}^{(k, \Gamma)}+[u]_{m, \alpha, \Omega}^{(k, \Gamma)},
\end{aligned}
$$


where $D^{\beta}:=\partial_{x_{1}}^{\beta_{1}} \ldots \partial_{x_{n}}^{\beta_{n}}$ for a multi-index $\beta=\left(\beta_{1}, \ldots, \beta_{n}\right)$ with $\beta_{i} \in \mathbb{Z}^{+}$for $i=1, \ldots, n$ and $|\beta|=\sum_{i=1}^{n} \beta_{i}$. We denote the completion of a set of smooth functions under $\|\cdot\|_{m, \alpha, \Omega}^{(k, \Gamma)}$ norm by $C_{(k, \Gamma)}^{m, \alpha}(\Omega)$.

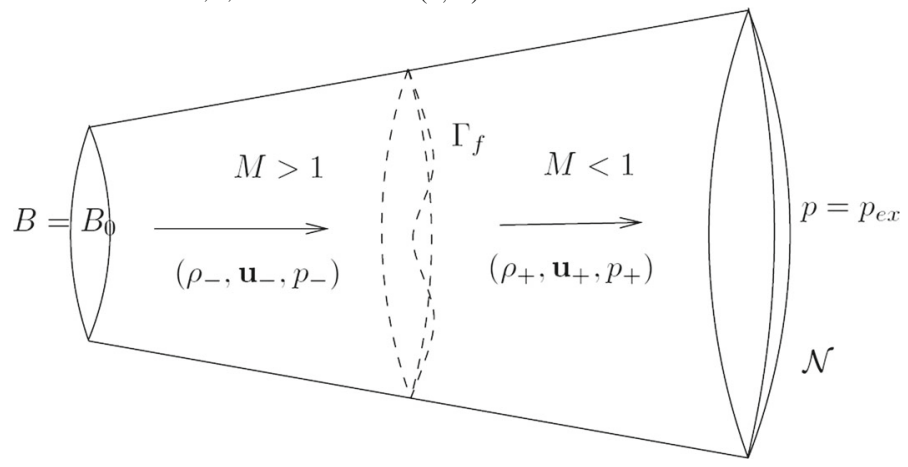

Our problem is given as follows:

Problem 1. (Transonic shock problem) Given an axisymmetric supersonic solution $\left(\rho_{-}, \mathbf{u}_{-}, p_{-}\right)$of $(2.1)$ in $\mathcal{N}$ satisfying the slip boundary condition

$$
\mathbf{u}_{-} \cdot \boldsymbol{n}_{w}=0 \quad \text { on } \quad \Gamma_{w}:=\partial \mathcal{N} \cap\left\{r_{0}<r<r_{1}, \theta=\theta_{1}\right\}
$$

where $\boldsymbol{n}_{w}$ is the unit normal vector on $\Gamma_{w}$,

$$
B=B_{0} \quad \text { on } \quad \Gamma_{e n}
$$

and

$$
\left\|\left(\rho_{-}, \mathbf{u}_{-}, p_{-}\right)-\left(\rho_{0}^{-}, u_{0}^{-} \mathbf{e}_{r}, p_{0}^{-}\right)\right\|_{2, \alpha, \mathcal{N}} \leq \sigma
$$

and an axisymmetric exit pressure $p_{e x}$ on $\Gamma_{e x}:=\partial \mathcal{N} \cap\left\{r=r_{1}, 0 \leq \theta<\theta_{1}\right\}$ satisfying

$$
\left\|p_{e x}-p_{c}\right\|_{1, \alpha, \Gamma_{e x}}^{\left(-\alpha, \partial \Gamma_{e x}\right)} \leq \sigma
$$

for a positive constant $\sigma$, find a shock location $\Gamma_{f}:=\mathcal{N} \cap\{r=f(\theta)\}$ and a corresponding subsonic solution $\left(\rho_{+}, \mathbf{u}_{+}, p_{+}\right) \in\left(C^{0}\left(\overline{\mathcal{N}_{f}^{+}}\right) \cap C^{1}\left(\mathcal{N}_{f}^{+}\right)\right)^{3}$ of $(2.1)$ satisfying

(i) the system (2.1) in $\mathcal{N}_{f}^{+}:=\mathcal{N} \cap\{r>f(\theta)\}$,

(ii) R-H conditions (2.3) on $\Gamma_{f}$,

(iii) the slip boundary condition

$$
\mathbf{u}_{+} \cdot \boldsymbol{n}_{w}=0 \quad \text { on } \quad \Gamma_{w}^{+}:=\Gamma_{w} \cap\left\{r>f\left(\theta_{1}\right)\right\},
$$

(iv) and the exit pressure condition

$$
p_{+}=p_{e x} \quad \text { on } \quad \Gamma_{e x} .
$$


Remark 3. It is generally known that a supersonic solution of (2.1) is governed by a hyperbolic system. We assume that $\left(\rho_{-}, \mathbf{u}_{-}, p_{-}\right)$in Problem 1 exists.

Remark 4. To simplify our argument, we assumed in Problem 1 that $\left(\rho_{-}, \mathbf{u}_{-}, p_{-}\right)$ satisfies (2.18). This assumption will be used to reduce (2.1) and (2.3) (see $\S 2.5$ ). The result for Problem 1 (Theorem 2) does not change if we consider a general perturbation of $\left(\rho_{0}^{-}, u_{0}^{-} \mathbf{e}_{r}, p_{0}^{-}\right)$in Problem 1.

We study Problem 1 using a stream function formulation of the full Euler system for an axisymmetric flow. We introduce a stream function formulation used in this paper in the next subsection.

\subsection{Stream function formulation}

Let $\Omega$ be an open simply connected axisymmetric set in $\mathbb{R}^{3}$. Let $(\rho, \mathbf{u})$ be axisymmetric $C^{1}$ functions in $\Omega$ satisfying the first equation of (2.1) and $|\rho \mathbf{u}|>0$. For such $(\rho, \mathbf{u})$, the Stokes' stream function for an axisymmetric flow of the full Euler system is defined by

$$
V(\mathrm{x})=\int_{\mathrm{S}_{\mathrm{x}}} \rho \mathbf{u} \cdot \boldsymbol{v} \mathrm{d} S \quad \text { for } \mathrm{x} \in \Omega,
$$

where $S_{\mathrm{x}}$ is a simply connected $C^{1}$ surface in $\Omega$ whose boundary is a circle centered at $z$-axis, parallel to $x y$-plane and passing through $\mathrm{x}$, and $\boldsymbol{v}$ is the unit normal vector on $S_{\mathrm{x}}$ pointing outward direction with respect to the cone-like domain made by connecting $\partial S_{\mathrm{x}}$ and the origin by straight lines. By the first equation of (2.1), the value of this function at $\mathrm{x}$ is independent of the choice of $S_{\mathrm{x}}$. Since $\partial S_{\mathrm{x}}$ is axisymmetric, $V$ is axisymmetric in $\Omega$.

By the first equation of (2.1), this function is a constant on each stream surface of $\rho \mathbf{u}$ in $\Omega$. Here, the stream surfaces of a vector field $\rho \mathbf{u}$ in $\Omega$ is a set of surfaces made by collecting all the streamlines of $\rho \mathbf{u}$ initiating from a point on a circle in $\Omega$ centered at $z$-axis and parallel to $x y$-plane. By $|\rho \mathbf{u}|>0$ in $\Omega, V$ on each different stream surface of vector field $\rho \mathbf{u}$ in $\Omega$ is different from each other.

Using these facts, a vector field in $\Omega$ tangential to the stream surfaces in $\Omega$ and having no $\mathbf{e}_{\varphi}$ component can be obtained from $V$. Let $\nabla^{\perp}=\frac{1}{2 \pi r \sin \theta}\left(\mathbf{e}_{r} \frac{\partial_{\theta}}{r}-\right.$ $\left.\mathbf{e}_{\theta} \partial_{r}\right)$. Then $\nabla^{\perp}$ satisfies $\nabla^{\perp} h \cdot \nabla h=0$ and $\nabla^{\perp} h \cdot \mathbf{e}_{\varphi}=0$ for a scalar function $h$. Using these properties of $\nabla^{\perp}$, we have that if we apply $\nabla^{\perp}$ to $V$, then we have a vector field in $\Omega$ tangent to the stream surfaces in $\Omega$ and having no $\mathbf{e}_{\varphi}$ component. Apply $\nabla^{\perp}$ to $V$. Then we have

$$
\nabla^{\perp} V=\rho u_{r} \mathbf{e}_{r}+\rho u_{\theta} \mathbf{e}_{\theta},
$$

where $u_{r}=\mathbf{u} \cdot \mathbf{e}_{r}$ and $u_{\theta}=\mathbf{u} \cdot \mathbf{e}_{\theta}$.

Using (2.24), we can reformulate the full Euler system for an axisymmetric flow. But if we do this, then there is a singularity issue that can be seen in the relation $\left\|\rho u_{r} \mathbf{e}_{r}+\rho u_{\theta} \mathbf{e}_{\theta}\right\|_{\alpha, \mathcal{N}}=\left\|\nabla^{\perp} V\right\|_{\alpha, \mathcal{N}} \nless \subset C\|V\|_{1, \alpha, \mathcal{N}}$ for any constant $C$. To avoid this issue, we use the following form of the stream function. 
Let $\Phi \mathbf{e}_{\varphi}$ be an axisymmetric vector field in $\Omega$ satisfying

$$
\oint_{\partial S_{\mathrm{x}}} \Phi \mathbf{e}_{\varphi} \cdot d \boldsymbol{r}=\int_{S_{\mathrm{x}}} \rho \mathbf{u} \cdot \boldsymbol{v} d S
$$

where $\boldsymbol{r}$ is a parametrization of $\partial S_{\mathrm{x}}$ in a counter clockwise direction. Then by the definitions of $\Phi \mathbf{e}_{\varphi}$ and $V$,

$$
\Phi=\frac{V}{2 \pi r \sin \theta}
$$

It is easily checked that

$$
\nabla \times\left(\Phi \mathbf{e}_{\varphi}\right)=\nabla^{\perp} V
$$

From this relation and (2.24) or (2.25) directly, we have

$$
\nabla \times\left(\Phi \mathbf{e}_{\varphi}\right)=\rho u_{r} \mathbf{e}_{r}+\rho u_{\theta} \mathbf{e}_{\theta} .
$$

We call $\Phi \mathbf{e}_{\varphi}$ the vector potential form of the stream function.

We reformulate the full Euler system for an axisymmetric flow using (2.28). For our later analysis, when we reformulate the full Euler system using (2.28), we use the following form of the full Euler system representing the relation between $\nabla S$ and $\nabla \times \mathbf{u}$ clearly

$$
\left\{\begin{array}{l}
\operatorname{div}(\rho \mathbf{u})=0, \\
(\nabla \times \mathbf{u}) \times \mathbf{u}=\frac{\rho^{\gamma-1}}{\gamma-1} \nabla S-\nabla B, \\
\rho \mathbf{u} \cdot \nabla B=0
\end{array}\right.
$$

which is obtained under the assumption that $(\rho, \mathbf{u}, p) \in C^{1}$ and $\rho>0$. We assume that $(\rho, \mathbf{u}, p)$ in (2.29) is axisymmetric and reformulate (2.29) using (2.28).

Rewrite (2.28) as $\mathbf{u}=\frac{1}{\rho} \nabla \times\left(\Phi \mathbf{e}_{\varphi}\right)+u_{\varphi} \mathbf{e}_{\varphi}$. Substitute this into the second equation of (2.29). Then we obtain

$$
\begin{aligned}
& \left(\nabla \times\left(\frac{1}{\rho} \nabla \times\left(\Phi \mathbf{e}_{\varphi}\right)+u_{\varphi} \mathbf{e}_{\varphi}\right)\right) \times\left(\frac{1}{\rho} \nabla \times\left(\Phi \mathbf{e}_{\varphi}\right)+u_{\varphi} \mathbf{e}_{\varphi}\right) \\
& =\frac{\rho^{\gamma-1}}{\gamma-1} \nabla S-\nabla B .
\end{aligned}
$$

From this equation, three equations can be obtained. From $\mathbf{e}_{\varphi}$-components of (2.30), we get

$$
\left(\nabla \times\left(u_{\varphi} \mathbf{e}_{\varphi}\right)\right) \times\left(\frac{1}{\rho} \nabla \times\left(\Phi \mathbf{e}_{\varphi}\right)\right)=0 .
$$

Define $L:=2 \pi r \sin \theta u_{\varphi}$ so that $\nabla \times\left(u_{\varphi} \mathbf{e}_{\varphi}\right)=\nabla^{\perp} L$ (see (2.26) and (2.27)). Then, using this relation, (2.31) can be written as

$$
\nabla^{\perp} L \times\left(\frac{1}{\rho} \nabla \times\left(\Phi \mathbf{e}_{\varphi}\right)\right)=0 .
$$


From this equation, we obtain

$$
\nabla \times\left(\Phi \mathbf{e}_{\varphi}\right) \cdot \nabla L=0 .
$$

From $\mathbf{e}_{\theta}$-components of (2.30), we get

$$
\begin{aligned}
& \left(\nabla \times\left(\frac{1}{\rho} \nabla \times\left(\Phi \mathbf{e}_{\varphi}\right)\right)\right) \times\left(\frac{1}{\rho} \nabla \times\left(\Phi \mathbf{e}_{\varphi}\right) \cdot \mathbf{e}_{r}\right) \mathbf{e}_{r}+\left(\nabla \times\left(u_{\varphi} \mathbf{e}_{\varphi}\right) \cdot \mathbf{e}_{r}\right) \mathbf{e}_{r} \times u_{\varphi} \mathbf{e}_{\varphi} \\
& =\left(\frac{\rho^{\gamma-1}}{\gamma-1} \frac{\partial_{\theta} S}{r}-\frac{\partial_{\theta} B}{r}\right) \mathbf{e}_{\theta} .
\end{aligned}
$$

Using the definition of $L$, rewrite the above equation. And then move the rewritten term into the right hand side of the equation and multiply $\mathbf{e}_{r} \times$ to the resultant equation. Then we obtain

$$
\begin{aligned}
& \left(\frac{1}{\rho} \nabla \times\left(\Phi \mathbf{e}_{\varphi}\right) \cdot \mathbf{e}_{r}\right) \nabla \times\left(\frac{1}{\rho} \nabla \times\left(\Phi \mathbf{e}_{\varphi}\right)\right) \\
& \quad=\left(\frac{L}{2 \pi r \sin \theta} \nabla \times\left(\frac{L}{2 \pi r \sin \theta} \mathbf{e}_{\varphi}\right) \cdot \mathbf{e}_{r}+\frac{\rho^{\gamma-1}}{\gamma-1} \frac{\partial_{\theta} S}{r}-\frac{\partial_{\theta} B}{r}\right) \mathbf{e}_{\varphi} .
\end{aligned}
$$

Using the third equation of (2.29), we obtain from $\mathbf{u}$-components of (2.30)

$$
\rho \mathbf{u} \cdot \nabla S=0 .
$$

Using the assumption that $(\rho, \mathbf{u}, p)$ are axisymmetric and (2.28), this equation can be written as

$$
\nabla \times\left(\Phi \mathbf{e}_{\varphi}\right) \cdot \nabla S=0
$$

Hence, we obtain from (2.30) three equations: (2.32), (2.33) and (2.35). Finally, in the same way that we obtained (2.35) from (2.34), we obtain from the third equation of (2.29)

$$
\nabla \times\left(\Phi \mathbf{e}_{\varphi}\right) \cdot \nabla B=0 .
$$

Combining (2.32), (2.33), (2.35) and (2.36), we have the following stream function formulation of the full Euler system for an axisymmetric flow

$$
\left\{\begin{array}{l}
\left(\frac{1}{\rho} \nabla \times\left(\Phi \mathbf{e}_{\varphi}\right) \cdot \mathbf{e}_{r}\right) \nabla \times\left(\frac{1}{\rho} \nabla \times\left(\Phi \mathbf{e}_{\varphi}\right)\right) \\
\quad=\left(\frac{L}{2 \pi r \sin \theta} \nabla \times\left(\frac{L}{2 \pi r \sin \theta} \mathbf{e}_{\varphi}\right) \cdot \mathbf{e}_{r}+\frac{\rho^{\gamma-1}}{\gamma-1} \frac{\partial_{\theta} S}{r}-\frac{\partial_{\theta} B}{r}\right) \mathbf{e}_{\varphi}, \\
\nabla \times\left(\Phi \mathbf{e}_{\varphi}\right) \cdot \nabla L=0 \\
\nabla \times\left(\Phi \mathbf{e}_{\varphi}\right) \cdot \nabla S=0 \\
\nabla \times\left(\Phi \mathbf{e}_{\varphi}\right) \cdot \nabla B=0
\end{array}\right.
$$

Note that the first equation of (2.1) is omitted in (2.37) because for a 3-D axisymmetric flow, the first equation of (2.1) is reduced to $\operatorname{div}\left(\rho u_{r} \mathbf{e}_{r}+\rho u_{\theta} \mathbf{e}_{\theta}\right)=0$ and this equation is directly satisfied by $\operatorname{div}\left(\nabla \times\left(\Phi \mathbf{e}_{\varphi}\right)\right)=0$ if $\Phi \in C^{2}(\Omega)$. Also, note that when $(\rho, \mathbf{u}, p)$ in (2.1) is in a sufficiently small perturbation of $\left(\rho_{0}^{+}, u_{0}^{+} \mathbf{e}_{r}, p_{0}^{+}\right)$ or $\left(\rho_{0}^{-}, u_{0}^{-} \mathbf{e}_{r}, p_{0}^{-}\right)$, then unknowns of (2.37) can be $\left(\Phi \mathbf{e}_{\varphi}, L, S, B\right)$. This fact (for the first case) is checked via the following lemma: 
Lemma 3. Let $\Omega$ be an axisymmetric connected open subset of $\mathcal{N}_{r_{s}-\delta_{1}}^{+}$. There exist positive constants $\delta_{2, \Omega}$ and $\delta_{3, \Omega}$ and a function $\varrho: B_{\delta_{2, \Omega}, \Omega}^{(1)} \rightarrow B_{\delta_{3, \Omega}, \Omega}^{(2)}$, where

$$
\begin{aligned}
B_{\delta, \Omega}^{(1)}:= & \left\{\left(\rho u_{r} \mathbf{e}_{r}+\rho u_{\theta} \mathbf{e}_{\theta}, u_{\varphi} \mathbf{e}_{\varphi}, S, B\right)\left|\in\left(C^{0}(\bar{\Omega})\right)^{4}\right|\right. \\
& \left.\sup _{\Omega}\left\{\left|\rho u_{r} \mathbf{e}_{r}+\rho u_{\theta} \mathbf{e}_{\theta}-\rho_{0}^{+} u_{0}^{+} \mathbf{e}_{r}\right|+\left|u_{\varphi} \mathbf{e}_{\varphi}\right|+\left|S-S_{0}^{+}\right|+\left|B-B_{0}\right|\right\} \leq \delta\right\},
\end{aligned}
$$

and

$$
B_{\delta, \Omega}^{(2)}:=\left\{\rho \in C^{0}(\bar{\Omega})\left|\sup _{\Omega}\right| \rho-\rho_{0}^{+} \mid \leq \delta\right\}
$$

such that

$$
\rho^{2} B-\frac{\left|\rho u_{r} \mathbf{e}_{r}+\rho u_{\theta} \mathbf{e}_{\theta}\right|^{2}}{2}-\frac{\rho^{2}}{2}\left|u_{\varphi} \mathbf{e}_{\varphi}\right|^{2}-\frac{\gamma S \rho^{\gamma+1}}{\gamma-1}=0
$$

if and only if

$$
\rho=\varrho\left(\rho u_{r} \mathbf{e}_{r}+\rho u_{\theta} \mathbf{e}_{\theta}, u_{\varphi} \mathbf{e}_{\varphi}, S, B\right),
$$

for all $\left(\rho u_{r} \mathbf{e}_{r}+\rho u_{\theta} \mathbf{e}_{\theta}, u_{\varphi} \mathbf{e}_{\varphi}, S, B\right) \in B_{\delta_{2, \Omega}, \Omega}^{(1)}$ and $\rho \in B_{\delta_{3, \Omega}, \Omega}^{(2)}$

Proof. Using the definition of $B$ given in (2.2), we define

$$
\begin{aligned}
& b\left(\rho, \rho u_{r} \mathbf{e}_{r}+\rho u_{\theta} \mathbf{e}_{\theta}, u_{\varphi} \mathbf{e}_{\varphi}, S, B\right) \\
& \quad=\rho^{2} B-\frac{\left|\rho u_{r} \mathbf{e}_{r}+\rho u_{\theta} \mathbf{e}_{\theta}\right|^{2}}{2}-\frac{\rho^{2}}{2}\left|u_{\varphi} \mathbf{e}_{\varphi}\right|^{2}-\frac{\gamma S \rho^{\gamma+1}}{\gamma-1} .
\end{aligned}
$$

Then $b \in C^{\infty}$ with respect to $\left(\rho, \rho u_{r} \mathbf{e}_{r}+\rho u_{\theta} \mathbf{e}_{\theta}, u_{\varphi} \mathbf{e}_{\varphi}, S, B\right)$,

$$
\partial_{\rho} b\left(\rho_{0}^{+}, \rho_{0}^{+} u_{0}^{+} \mathbf{e}_{r}, 0, S_{0}^{+}, B_{0}\right)=\rho_{0}^{+}\left(u_{0}^{+2}-\gamma S_{0}^{+} \rho_{0}^{+\gamma-1}\right)<0,
$$

and by the third equation of (2.14),

$$
b\left(\rho_{0}^{+}, \rho_{0}^{+} u_{0}^{+} \mathbf{e}_{r}, 0, S_{0}^{+}, B_{0}\right)=0 .
$$

With these facts, we apply the implicit function theorem to $b\left(\rho, \rho u_{r} \mathbf{e}_{r}+\rho u_{\theta} \mathbf{e}_{\theta}\right.$, $\left.u_{\varphi} \mathbf{e}_{\varphi}, S, B\right)$. Then we obtain the desired result.

Remark 5. Hereafter, $\delta_{2}$ and $\delta_{3}$ denote some constants $\delta_{2, \Omega}$ and $\delta_{3, \Omega}$ in Lemma 3 for $\Omega=\mathcal{N}_{r_{s}-\delta_{1}}^{+}$. Hereafter, $\varrho$ denotes $\varrho$ in Lemma 3 for $\Omega=\mathcal{N}_{r_{s}-\delta_{1}}^{+}$. One can see that if $\Omega \subset \mathcal{N}_{r_{s}-\delta_{1}}^{+}$, then for all $\left(\nabla \times\left(\Phi \mathbf{e}_{\varphi}\right), \frac{L}{2 \pi r \sin \theta} \mathbf{e}_{\varphi}, S, B\right) \in B_{\delta_{2}, \Omega}^{(1)}$ and $\rho \in B_{\delta_{3}, \Omega}^{(2)}$, (2.38) holds if and only if (2.39) holds. 
In order to study 3-D axisymmetric transonic shock solution of the full Euler system using the stream function formulation of the full Euler system for an axisymmetric flow above, we reformulate (2.3) with respect to the variables in (2.37).

Assume that $\Gamma$ in (2.3) is an axisymmetric $C^{1}$ surface, and let $\tau_{2}$ and $\tau_{1}$ in (2.3) be $\mathbf{e}_{\varphi}$ and the unit tangent vector field on $\Gamma$ perpendicular to $\mathbf{e}_{\varphi}$ and satisfying $\boldsymbol{v} \cdot\left(\boldsymbol{\tau}_{1} \times \mathbf{e}_{\varphi}\right)>0$, respectively, where $\boldsymbol{v}$ is the unit normal vector field on $\Gamma$ pointing toward $\Omega^{+}$. We reformulate this (2.3) with respect to the variables in (2.37).

By the definition of $V$ given in (2.23), the first equation of (2.3) can be written as $[V]_{\Gamma}=0$. Rewrite this equation using (2.26). Then we have

$$
\left[\Phi \mathbf{e}_{\varphi}\right]_{\Gamma}=0 .
$$

Using (2.28), rewrite the second equation of (2.3). Then we get

$$
\frac{1}{\rho} \nabla \times\left(\Phi \mathbf{e}_{\varphi}\right) \cdot \boldsymbol{\tau}_{1}=\mathbf{u}_{-} \cdot \boldsymbol{\tau}_{1} \text { on } \Gamma .
$$

By the definition of $L$, the third equation of (2.3) can be written as

$$
[L]_{\Gamma}=0 .
$$

From the second, third and fifth equation of (2.3), we can obtain

$$
\left[B_{S}\right]_{\Gamma}=0
$$

where $B_{s}:=\frac{(\mathbf{u} \cdot v)^{2}}{2}+\frac{\gamma p}{(\gamma-1) \rho}$. In the same way that (2.12) is obtained from (2.8), we obtain from the first and fourth equation of (2.3) and (2.41)

$$
S_{+}=g\left(\left(\frac{\mathbf{u}_{-} \cdot \boldsymbol{v}}{c_{-}}\right)^{2}\right) S_{-} \text {on } \Gamma,
$$

where $g(x)$ is a function defined in (2.13) and variables with lower indices \pm denote variables in $\Omega^{ \pm}$, respectively. Combining these reformulated equations of (2.3) and the fifth equation of (2.3), we have the following stream function formulation of (2.3):

$$
\left\{\begin{array}{l}
{\left[\Phi \mathbf{e}_{\varphi}\right]_{\Gamma}=0,} \\
\frac{1}{\rho} \nabla \times\left(\Phi \mathbf{e}_{\varphi}\right) \cdot \boldsymbol{\tau}_{1}=\mathbf{u}_{-} \cdot \boldsymbol{\tau}_{1} \text { on } \Gamma, \\
{[L]_{\Gamma}=0,} \\
S_{+}=g\left(\left(\frac{\mathbf{u}_{-} \cdot \boldsymbol{v}}{c_{-}}\right)^{2}\right) S_{-} \text {on } \Gamma, \\
{[B]_{\Gamma}=0 .}
\end{array}\right.
$$




\subsection{Restatement of Problem 1 using the stream function formulation and main result}

Using the stream function formulation in the previous subsection, we restate Problem 1.

We first reduce (2.1) and (2.3) in Problem 1. In Problem 1, we assumed that a supersonic solution $\left(\rho_{-}, \mathbf{u}_{-}, p_{-}\right)$of (2.1) satisfies (2.18). If this condition is satisfied, then by the third equation of (2.1) in $\mathcal{N}_{f}^{-}:=\mathcal{N} \cap\{r<f(\theta)\}$ and $\mathcal{N}_{f}^{+}$, the fifth equation of $(2.3),(2.17)$ and $(2.21),\left(\rho_{+}, \mathbf{u}_{+}, p_{+}\right)$we find in Problem 1 must satisfy $B=B_{0}$ in $\overline{\mathcal{N}_{f}^{+}}$. If the fact that the third equation of (2.1) in our case has a unique sufficiently regular solution is guaranteed, then it is not needed to solve the third equation of (2.1) to obtain $B=B_{0}$ in $\overline{\mathcal{N}_{f}^{+}}$. (The fact that the third equation of (2.1) in our case has a unique sufficiently regular solution is shown by Lemma 15.) To simplify our argument, we assume that $\left(\rho_{+}, \mathbf{u}_{+}, p_{+}\right)$in Problem 1 satisfies $B=B_{0}$ in $\overline{\mathcal{N}_{f}^{+}}$. Under this assumption, (2.1) and (2.3) that $\left(\rho_{+}, \mathbf{u}_{+}, p_{+}\right)$ in Problem 1 satisfies are reduced to the first and second equation of (2.1) and the first, second, third and fourth equation of (2.3).

Then we present the stream function formulations of (2.1) and (2.3) satisfied by $\left(\rho_{+}, \mathbf{u}_{+}, p_{+}\right)$in Problem 1. By (2.37) and (2.42), the stream function formulations of (2.1) and (2.3) satisfied by $\left(\rho_{+}, \mathbf{u}_{+}, p_{+}\right)$in Problem 1 and reduced by using the assumption that $\left(\rho_{+}, \mathbf{u}_{+}, p_{+}\right)$satisfies $B=B_{0}$ in $\overline{\mathcal{N}_{f}^{+}}$are given as

$$
\begin{aligned}
& \left(\frac{1}{\rho_{+}} \nabla \times\left(\Phi_{+} \mathbf{e}_{\varphi}\right) \cdot \mathbf{e}_{r}\right) \nabla \times\left(\frac{1}{\rho_{+}} \nabla \times\left(\Phi_{+} \mathbf{e}_{\varphi}\right)\right) \\
& \quad=\left(\frac{L_{+}}{2 \pi r \sin \theta} \nabla \times\left(\frac{L_{+}}{2 \pi r \sin \theta} \mathbf{e}_{\varphi}\right) \cdot \mathbf{e}_{r}+\frac{\rho_{+}^{\gamma-1}}{\gamma-1} \frac{\partial_{\theta} S_{+}}{r}\right) \mathbf{e}_{\varphi}, \\
& \nabla \times\left(\Phi_{+} \mathbf{e}_{\varphi}\right) \cdot \nabla L_{+}=0, \\
& \nabla \times\left(\Phi_{+} \mathbf{e}_{\varphi}\right) \cdot \nabla S_{+}=0
\end{aligned}
$$

in $\mathcal{N}_{f}^{+}$and

$$
\begin{aligned}
& \Phi_{+} \mathbf{e}_{\varphi}=\Phi_{-} \mathbf{e}_{\varphi} \text { on } \Gamma_{f}, \\
& \frac{1}{\rho_{+}} \nabla \times\left(\Phi_{+} \mathbf{e}_{\varphi}\right) \cdot \boldsymbol{\tau}_{f}=\mathbf{u}_{-} \cdot \boldsymbol{\tau}_{f} \text { on } \Gamma_{f}, \\
& L_{+}=L_{-} \text {on } \Gamma_{f}, \\
& S_{+}=g\left(\left(\frac{\mathbf{u}_{-} \cdot \boldsymbol{v}_{f}}{c_{-}}\right)^{2}\right) S_{-} \text {on } \Gamma_{f},
\end{aligned}
$$

where $\boldsymbol{v}_{f}$ is the unit normal vector on $\Gamma_{f}$ pointing toward $\mathcal{N}_{f}^{+}$and $\boldsymbol{\tau}_{f}$ is the unit tangential vector on $\Gamma_{f}$ perpendicular to $\mathbf{e}_{\varphi}$ and satisfying $\boldsymbol{v}_{f} \cdot\left(\boldsymbol{\tau}_{f} \times \mathbf{e}_{\varphi}\right)>0$. Here, $\left(\Phi_{-} \mathbf{e}_{\varphi}, L_{-}, S_{-}\right)$and $\left(\Phi_{+} \mathbf{e}_{\varphi}, L_{+}, S_{+}\right)$are $\left(\Phi \mathbf{e}_{\varphi}, L, S\right)$ given by the definitions of $\Phi \mathbf{e}_{\varphi}, L$ and $S$ for $(\rho, \mathbf{u}, p)=\left(\rho_{-}, \mathbf{u}_{-}, p_{-}\right)$and $\left(\rho_{+}, \mathbf{u}_{+}, p_{+}\right)$, respectively.

To use (2.43)-(2.49) to solve Problem 1, we determine $\rho_{+}$in (2.43) and (2.47). In Problem 1 , we will consider the case where $\left(\rho_{-}, \mathbf{u}_{-}, p_{-}\right)$and $p_{e x}$ are in sufficiently 
small perturbations of $\left(\rho_{0}^{-}, u_{0}^{-} \mathbf{e}_{r}, p_{0}^{-}\right)$and $p_{c}$ so that $\left(\rho_{+}, \mathbf{u}_{+}, p_{+}\right)$we will find in Problem 1 satisfies that $\rho_{+}$is uniquely determined by $\left(\rho_{+} u_{+, r} \mathbf{e}_{r}+\rho_{+} u_{+, \theta} \mathbf{e}_{\theta}\right.$, $\left.u_{+, \varphi} \mathbf{e}_{\varphi}, S_{+}, B_{0}\right)$. (If $\left(\rho_{-}, \mathbf{u}_{-}, p_{-}\right)$and $p_{e x}$ are in sufficiently small perturbations of $\left(\rho_{0}^{-}, u_{0}^{-} \mathbf{e}_{r}, p_{0}^{-}\right)$and $p_{c}$, then it is expected that the solution $\left(\rho_{+}, \mathbf{u}_{+}, p_{+}\right)$in Problem 1 is in a small perturbation of $\left(\rho_{0}^{+}, u_{0}^{+} \mathbf{e}_{r}, p_{0}^{+}\right)$. And if $\left(\rho_{+}, \mathbf{u}_{+}, p_{+}\right)$is in a small perturbation of $\left(\rho_{0}^{+}, u_{0}^{+} \mathbf{e}_{r}, p_{0}^{+}\right)$, then $\rho_{+}$can be uniquely determined by $\left(\rho_{+} u_{+, r} \mathbf{e}_{r}+\rho_{+} u_{+, \theta} \mathbf{e}_{\theta}, u_{+, \varphi} \mathbf{e}_{\varphi}, S_{+}, B_{0}\right)$ where $u_{+, r}:=\mathbf{u}_{+} \cdot \mathbf{e}_{r}, u_{+, \theta}:=\mathbf{u}_{+} \cdot \mathbf{e}_{\theta}$ and $u_{+, \varphi}=\mathbf{u}_{+} \cdot \mathbf{e}_{\varphi}$ (see Lemma 3). ) To find such ( $\left.\rho_{+}, \mathbf{u}_{+}, p_{+}\right)$using (2.43)-(2.49), we set $\rho_{+}$in (2.43) and (2.47) to be

$$
\rho_{+}=\varrho\left(\nabla \times\left(\Phi_{+} \mathbf{e}_{\varphi}\right), \frac{L_{+}}{2 \pi r \sin \theta} \mathbf{e}_{\varphi}, S_{+}, B_{0}\right),
$$

where $\varrho$ is a function given in Lemma 3 . Hereafter, to simplify notation, we write $\varrho\left(\nabla \times\left(\Phi \mathbf{e}_{\varphi}\right), \frac{L}{2 \pi r \sin \theta} \mathbf{e}_{\varphi}, S, B_{0}\right)$ as $\varrho\left(\nabla \times\left(\Phi \mathbf{e}_{\varphi}\right), \frac{L}{2 \pi r \sin \theta} \mathbf{e}_{\varphi}, S\right)$.

We will find a subsonic solution of (2.1) using (2.43)-(2.45). To do this, we define a subsonic solution of (2.43)-(2.45). Using the definition of subsonic solution of (2.1), we define a subsonic solution of (2.43)-(2.45) by a solution $\left(\Phi_{+} \mathbf{e}_{\varphi}, L_{+}, S_{+}\right)$ of (2.43)-(2.45) satisfying

$$
\begin{aligned}
& \left|\frac{1}{\varrho\left(\nabla \times\left(\Phi_{+} \mathbf{e}_{\varphi}\right), \frac{L_{+}}{2 \pi r \sin \theta} \mathbf{e}_{\varphi}, S_{+}\right)} \nabla \times\left(\Phi_{+} \mathbf{e}_{\varphi}\right)+\frac{L_{+}}{2 \pi r \sin \theta} \mathbf{e}_{\varphi}\right|^{2} \\
& \quad<\gamma S_{+}\left(\varrho\left(\nabla \times\left(\Phi_{+} \mathbf{e}_{\varphi}\right), \frac{L_{+}}{2 \pi r \sin \theta} \mathbf{e}_{\varphi}, S_{+}\right)\right)^{\gamma-1} .
\end{aligned}
$$

Next, we rewrite the boundary conditions in Problem 1 with respect to the variables in (2.37). Denote $V$ given by the definition of $V$ for $(\rho, \mathbf{u}, p)=\left(\rho_{-}, \mathbf{u}_{-}, p_{-}\right)$ and $\left(\rho_{+}, \mathbf{u}_{+}, p_{+}\right)$by $V_{-}$and $V_{+}$, respectively. By (2.24), (2.17) and (2.21) can be written as $V_{-}=V_{-}\left(r_{0}, \theta_{1}\right)$ on $\Gamma_{w}$ and $V_{+}=V_{+}\left(f\left(\theta_{1}\right), \theta_{1}\right)$ on $\Gamma_{w}^{+}$. Rewrite this in the vector potential form. Then we have $\Phi_{-} \mathbf{e}_{\varphi}=\frac{r_{0} \Phi_{-}\left(r_{0}, \theta_{1}\right)}{r} \mathbf{e}_{\varphi}$ on $\Gamma_{w}$ and $\Phi_{+} \mathbf{e}_{\varphi}=\frac{f\left(\theta_{1}\right) \Phi_{+}\left(f\left(\theta_{1}\right), \theta_{1}\right)}{r} \mathbf{e}_{\varphi}$ on $\Gamma_{w}^{+}$. Combine these relations with (2.46) and the continuity condition for $\Phi \mathbf{e}_{\varphi}$ at $\overline{\Gamma_{f}} \cap \overline{\Gamma_{w}^{+}}$. Then we obtain

$$
\Phi_{+} \mathbf{e}_{\varphi}=\frac{r_{0} \Phi_{-}\left(r_{0}, \theta_{1}\right)}{r} \mathbf{e}_{\varphi} \text { on } \Gamma_{w}^{+} .
$$

Using the definition of $S$ and (2.50), rewrite (2.22). Then we have

$$
S_{+}\left(\varrho\left(\nabla \times\left(\Phi_{+} \mathbf{e}_{\varphi}\right), \frac{L_{+}}{2 \pi r \sin \theta} \mathbf{e}_{\varphi}, S_{+}\right)\right)^{\gamma}=p_{e x} \quad \text { on } \Gamma_{e x} .
$$

Finally, we add some continuity condition for $\Phi_{+} \mathbf{e}_{\varphi}$ in the restatement of Problem 1 using the stream function formulation. By (2.1) and $(2.21),\left(\rho_{+}, \mathbf{u}_{+}\right)$we find in Prolem 1 must satisfy that the total outgoing flux for $\rho_{+} \mathbf{u}_{+}$on $\Gamma_{e x}$ is equal to the total incoming flux for $\rho_{+} \mathbf{u}_{+}$on any cross section of $\mathcal{N}_{f}^{+}$whose all boundary points intersect with $\Gamma_{w}^{+}$. Using $(2.23)$, this statement can be expressed as 
$\lim _{\theta \rightarrow \theta_{1}} V_{+}\left(r_{1}, \theta\right)=\lim _{r \rightarrow r_{1}} V_{+}\left(r, \theta_{1}\right)$. In the vector potential form, this can be rewritten as

$$
\lim _{\theta \rightarrow \theta_{1}}\left(\Phi_{+} \mathbf{e}_{\varphi}\right)\left(r_{1}, \theta\right)=\lim _{r \rightarrow r_{1}}\left(\Phi_{+} \mathbf{e}_{\varphi}\right)\left(r, \theta_{1}\right) .
$$

Since this condition cannot be achieved by finding a solution satisfying (2.53) (this will be seen in $\S 3.1$ ), we include this condition in the restatement of Problem 1 using the stream function formulation.

Using the equations and boundary conditions obtained above, Problem 1 is restated as follows:

Problem 2. Given an axisymmetric supersonic solution $\left(\rho_{-}, \mathbf{u}_{-}, p_{-}\right)$of (2.1) and an axisymmetric exit pressure $p_{e x}$ as in Problem 1, find a shock location $\Gamma_{f}=$ $\mathcal{N} \cap\{r=f(\theta)\}$ and a corresponding subsonic solution $\left(\Phi_{+} \mathbf{e}_{\varphi}, L_{+}, S_{+}\right)$of (2.43)(2.45) satisfying

(i) the system (2.43)-(2.45) in $\mathcal{N}_{f}^{+}$,

(ii) the R-H conditions (2.46)-(2.49),

(iii) the slip boundary condition (2.52),

(iv) the exit pressure condition (2.53),

(v) the compatibility condition (2.54).

Let $S^{2, \theta_{1}}:=\left\{(x, y, z) \in \mathbb{R}^{3} \mid r=1,0 \leq \theta<\theta_{1}\right\}$. Then a function $f$ representing a shock location $\Gamma_{f}$ can be considered as a function on $S^{2, \theta_{1}}$. Using this fact and the stereographic projection from $(0,0,-1)$ onto the plane $z=1$ passing through $S^{2, \theta_{1}}$, it is seen that $f$ can be regarded as a function on $\Lambda$ where $\Lambda:=\left\{(x, y) \in \mathbb{R}^{2} \mid \sqrt{x^{2}+y^{2}}<2 \tan \frac{\theta_{1}}{2}\right\}$. Thus, $f$ can be regarded as a function on $\Lambda$ or $\left(0, \theta_{1}\right)$. In this paper, we regard $f$ in both ways. To simplify our notation, we use the same function notation when we represent $f$ as a function on $\Lambda$ or $\left(0, \theta_{1}\right)$.

Hereafter, we denote $\Phi \mathbf{e}_{\varphi}, L$ and $V$ given by the definitions of $\Phi \mathbf{e}_{\varphi}, L$ and $V$ for $(\rho, \mathbf{u}, p)=\left(\rho_{0}^{ \pm}, u_{0}^{ \pm} \mathbf{e}_{r}, p_{0}^{ \pm}\right)$by $\Phi_{0}^{ \pm} \mathbf{e}_{\varphi}, L_{0}^{ \pm}$and $V_{0}^{ \pm}$, respectively. To simplify our notation, hereafter, we denote $\left(\Phi_{+} \mathbf{e}_{\varphi}, L_{+}, S_{+}\right)$by $\left(\Phi \mathbf{e}_{\varphi}, L, S\right)$.

Our result of Problem 2, the main result in this paper, is given as follows.

Theorem 1. Let $\alpha \in\left(\frac{2}{3}, 1\right)$. There exists a positive constant $\sigma_{1}$ depending on $\left(\rho_{i n}, u_{i n}, p_{i n}\right), p_{c}, \gamma, r_{0}, r_{1}, \theta_{1}$ and $\alpha$ such that if $\sigma \in\left(0, \sigma_{1}\right]$, then Problem 2 has a solution $\left(f, \Phi \mathbf{e}_{\varphi}, L, S\right)$ satisfying the estimate

$$
\begin{aligned}
& \left\|f-r_{s}\right\|_{2, \alpha, \Lambda}^{(-1-\alpha, \partial \Lambda)}+\left\|\nabla \times\left(\left(\Phi-\Phi_{0}^{+}\right) \mathbf{e}_{\varphi}\right)\right\|_{1, \alpha, \mathcal{N}_{f}^{+}}^{\left(-\alpha, \Gamma_{w}^{+}\right)} \\
& +\left\|\frac{L}{2 \pi r \sin \theta} \mathbf{e}_{\varphi}\right\|_{1, \alpha, \mathcal{N}_{f}^{+}}^{\left(-\alpha, \Gamma_{+}^{+}\right)}+\left\|S-S_{0}^{+}\right\|_{1, \alpha, \mathcal{N}_{f}^{+}}^{\left(-\alpha, \Gamma_{w}^{+}\right)} \leq C \sigma
\end{aligned}
$$

where $C$ is a positive constant depending on $\left(\rho_{i n}, u_{i n}, p_{i n}\right), p_{c}, \gamma, r_{0}, r_{1}, \theta_{1}$ and $\alpha$. Furthermore, this solution is unique in the class of functions satisfying (2.55).

Remark 6. The condition $\alpha \in\left(\frac{2}{3}, 1\right)$ is a sufficient condition for obtaining $C^{1, \alpha}$ up to boundary estimate of solutions of the elliptic part of the linearized equations of (2.43)-(2.49), (2.52)-(2.54) (see the proof of Lemma 11). We do not know that this is an optimal condition. 
Hereafter, we say that a constant depends on the data if a constant depends on $\left(\rho_{i n}, u_{i n}, p_{i n}\right), p_{c}, \gamma, r_{0}, r_{1}, \theta_{1}$ and $\alpha$.

The following result of Problem 1 is obtained from Theorem 1.

Theorem 2. Let $\alpha \in\left(\frac{2}{3}, 1\right)$. There exists a positive constant $\sigma_{2}$ depending on the data such that if $\sigma \in\left(0, \sigma_{2}\right]$, then Problem 1 has a solution $\left(f, \rho_{+}, \mathbf{u}_{+}, p_{+}\right)$ satisfying the estimate

$$
\begin{aligned}
& \left\|f-r_{s}\right\|_{2, \alpha, \Lambda}^{(-1-\alpha, \partial \Lambda)} \\
& \quad+\left\|\rho_{+}-\rho_{0}^{+}\right\|_{1, \alpha, \mathcal{N}_{f}^{+}}^{\left(-\alpha, \Gamma_{w}^{+}\right)}+\left\|\mathbf{u}_{+}-u_{0}^{+} \mathbf{e}_{r}\right\|_{1, \alpha, \mathcal{N}_{f}^{+}}^{\left(-\alpha, \Gamma_{w}^{+}\right)}+\| p_{+} \\
& \quad-p_{0}^{+} \|_{1, \alpha, \mathcal{N}_{f}^{+}}^{\left(-\alpha, \Gamma_{w}^{+}\right)} \leq C \sigma
\end{aligned}
$$

where $C$ is a positive constant depending on the data. Furthermore, this solution is unique in the class of functions satisfying (2.56).

Proof. 1. Let $\left(f, \Phi \mathbf{e}_{\varphi}, L, S\right)$ be a solution of Problem 2 given in Theorem 1 for $\sigma \in\left(0, \bar{\sigma}_{2}\right]$ where $\bar{\sigma}_{2}$ is a positive constant less than or equal to $\sigma_{1}$ and to be determined later. Then since $\left(f, \Phi \mathbf{e}_{\varphi}, L, S\right)$ is a solution of Problem 2, $\varrho\left(\nabla \times\left(\Phi \mathbf{e}_{\varphi}\right), \frac{L}{2 \pi r \sin \theta} \mathbf{e}_{\varphi}, S\right)$ is well-defined in $\overline{\mathcal{N}_{f}^{+}}$. Define $\rho_{+}:=\varrho\left(\nabla \times\left(\Phi \mathbf{e}_{\varphi}\right)\right.$, $\left.\frac{L}{2 \pi r \sin \theta} \mathbf{e}_{\varphi}, S\right), \mathbf{u}_{+}:=\frac{\nabla \times\left(\Phi \mathbf{e}_{\varphi}\right)}{\varrho\left(\nabla \times\left(\Phi \mathbf{e}_{\varphi}\right), \frac{L}{2 \pi r \sin \theta} \mathbf{e}_{\varphi}, S\right)}$ and $p_{+}:=S\left(\varrho\left(\nabla \times\left(\Phi \mathbf{e}_{\varphi}\right)\right.\right.$, $\left.\left.\frac{L}{2 \pi r \sin \theta} \mathbf{e}_{\varphi}, S\right)\right)^{\gamma}$. Then since $\left(\Phi \mathbf{e}_{\varphi}, L, S\right)$ satisfies (2.43)-(2.45) in $\mathcal{N}_{f}^{+},(2.46)-$ (2.49), (2.52), (2.53), and

$b\left(\varrho\left(\nabla \times\left(\Phi \mathbf{e}_{\varphi}\right), \frac{L}{2 \pi r \sin \theta} \mathbf{e}_{\varphi}, S\right), \nabla \times\left(\Phi \mathbf{e}_{\varphi}\right), \frac{L}{2 \pi r \sin \theta} \mathbf{e}_{\varphi}, S, B_{0}\right)=0$ in $\overline{\mathcal{N}_{f}^{+}}$,

where $b$ is a funtion defined in (2.40), $\left(\rho_{+}, \mathbf{u}_{+}, p_{+}\right)$satisfies the second and third equation of (2.1) in $\mathcal{N}_{f}^{+},(2.3)$ on $\Gamma_{f},(2.21)$ and (2.22). Furthermore, since $\nabla \cdot\left(\nabla \times\left(\Phi \mathbf{e}_{\varphi}\right)\right)=0$ and $\nabla \cdot\left(\varrho\left(\nabla \times\left(\Phi \mathbf{e}_{\varphi}\right), \frac{L}{2 \pi r \sin \theta} \mathbf{e}_{\varphi}, S\right) \frac{L}{2 \pi r \sin \theta} \mathbf{e}_{\varphi}\right)=0$ in $\mathcal{N}_{f}^{+}$, $\left(\rho_{+}, \mathbf{u}_{+}, p_{+}\right)$satisfies the first equation of (2.1) in $\mathcal{N}_{f}^{+}$. Since $\left(\Phi \mathbf{e}_{\varphi}, L, S\right)$ is a subsonic solution of (2.43)-(2.45), $\left(\rho_{+}, \mathbf{u}_{+}, p_{+}\right)$is a subsonic solution of (2.1). Since $\left(\nabla \times\left(\Phi \mathbf{e}_{\varphi}\right), \frac{L}{2 \pi r \sin \theta} \mathbf{e}_{\varphi}, S\right) \in C_{\left(-\alpha, \Gamma_{w}^{+}\right)}^{1, \alpha}\left(\mathcal{N}_{f}^{+}\right),\left(\rho_{+}, \mathbf{u}_{+}, p_{+}\right) \in C_{\left(-\alpha, \Gamma_{w}^{+}\right)}^{1, \alpha}\left(\mathcal{N}_{f}^{+}\right)$. From these facts, we have that $\left(f, \rho_{+}, \mathbf{u}_{+}, p_{+}\right)$is a solution of Problem 1.

Obtain (2.56). By Lemma $3, \rho_{0}^{+}$can be written as $\rho_{0}^{+}=\varrho\left(\nabla \times\left(\Phi_{0}^{+} \mathbf{e}_{\varphi}\right)\right.$, $\left.\frac{L_{0}^{+}}{2 \pi r \sin \theta} \mathbf{e}_{\varphi}(=0), S_{0}^{+}\right)$. Using this expression, we write $\rho_{+}-\rho_{0}^{+}$as

$$
\begin{gathered}
\int_{0}^{1} \nabla \varrho\left(t\left(\nabla \times\left(\Phi \mathbf{e}_{\varphi}\right), \frac{L}{2 \pi r \sin \theta} \mathbf{e}_{\varphi}, S\right)+(1-t)\left(\nabla \times\left(\Phi_{0}^{+} \mathbf{e}_{\varphi}\right), 0, S_{0}^{+}\right)\right) \mathrm{d} t \\
\left(\nabla \times\left(\left(\Phi-\Phi_{0}^{+}\right) \mathbf{e}_{\varphi}\right), \frac{L}{2 \pi r \sin \theta} \mathbf{e}_{\varphi}, S-S_{0}^{+}\right) .
\end{gathered}
$$

Since $b$ is a $C^{\infty}$ function of $\left(\rho, \nabla \times\left(\Phi \mathbf{e}_{\varphi}\right), \frac{L}{2 \pi r \sin \theta} \mathbf{e}_{\varphi}, S, B\right), \varrho$ is a $C^{\infty}$ function of $\left(\nabla \times\left(\Phi \mathbf{e}_{\varphi}\right), \frac{L}{2 \pi r \sin \theta} \mathbf{e}_{\varphi}, S, B\right)$. With this fact, the fact that $\left(\rho_{0}^{+}, u_{0}^{+} \mathbf{e}_{r}, p_{0}^{+}\right) \in$ 
$\left(C^{\infty}\left(\overline{\mathcal{N}_{f}^{+}}\right)\right)^{3}$ and (2.55) satisfied by $\left(f, \Phi \mathbf{e}_{\varphi}, L, S\right)$ for $\sigma \in\left(0, \bar{\sigma}_{2}\right]$ for $\bar{\sigma}_{2} \leq \sigma_{1}$, we estimate (2.57) in $C_{\left(-\alpha, \Gamma_{w}^{+}\right)}^{1, \alpha}\left(\mathcal{N}_{f}^{+}\right)$. Then we obtain

$$
\left\|\rho_{+}-\rho_{0}^{+}\right\|_{1, \alpha, \mathcal{N}_{f}^{+}}^{\left(-\alpha, \Gamma^{+}\right)} \leq C \sigma
$$

where $C$ is a positive constant depending on the data. By this estimate, there exists a positive constant $\bar{\sigma}_{2}^{(1)}$ depending on the data such that if $\sigma \in\left(0, \bar{\sigma}_{2}^{(1)}\right]$, then

$$
\sup _{\mathcal{N}_{f}^{+}}\left|\frac{1}{\rho_{+}}\right| \leq C,
$$

where $C$ is a positive constant depending on the data. Take $\bar{\sigma}_{2}=\min \left(\sigma_{1}, \bar{\sigma}_{2}^{(1)}\right)$ so that (2.59) holds. With (2.55) satisfied by $\left(f, \Phi \mathbf{e}_{\varphi}, L, S\right),(2.58)$ and (2.59), we estimate $\mathbf{u}_{+}-u_{0}^{+} \mathbf{e}_{r}$ and $p_{+}-p_{0}^{+}$in $C_{\left(-\alpha, \Gamma_{w}^{+}\right)}^{1, \alpha}\left(\mathcal{N}_{f}^{+}\right)$. Then we obtain

$$
\left\|\mathbf{u}_{+}-u_{0}^{+} \mathbf{e}_{r}\right\|_{1, \alpha, \mathcal{N}_{f}^{+}}^{\left(-\alpha, \Gamma^{+}\right)}+\left\|p_{+}-p_{0}^{+}\right\|_{1, \alpha, \mathcal{N}_{f}^{+}}^{\left(-\alpha, \Gamma_{w}^{+}\right)} \leq C \sigma,
$$

where $C$ is a positive constant depending on the data. Combining this estimate, (2.55) and (2.58), we obtain (2.56).

2. Assume that for $\sigma \in\left(0, \underline{\sigma}_{2}\right]$ where $\underline{\sigma}_{2}$ is a positive constant to be determined later, there exist two solutions $\left(f^{i}, \rho_{+}^{i}, \mathbf{u}_{+}^{i}, p_{+}^{i}\right)$ for $i=1,2$ of Problem 1 satisfying the estimate (2.56). There exists a positive constant $\underline{\sigma}_{2}^{(1)}$ depending on the data such that if $\sigma \in\left(0, \underline{\sigma}_{2}^{(1)}\right]$, then 1$)$

$$
\sup _{\mathcal{N}_{f_{i}}^{+}}\left|\frac{1}{\rho_{+}^{i}}\right| \leq C
$$

for $i=1,2$ where $C$ is a positive constant depending on the data and 2) $\rho_{+}^{i}$ for $i=1,2$ are uniquely determined by $\left(\rho_{+}^{i}\left(u_{+, r}^{i} \mathbf{e}_{r}+u_{+, \theta}^{i} \mathbf{e}_{\theta}\right), u_{+, \varphi}^{i} \mathbf{e}_{\varphi}, S_{+}^{i}, B_{0}\right)$ where $u_{+, r}^{i}:=\mathbf{u}_{+}^{i} \cdot \mathbf{e}_{r}, u_{+, \theta}^{i}:=\mathbf{u}_{+}^{i} \cdot \mathbf{e}_{\theta}, u_{+, \varphi}^{i}:=\mathbf{u}_{+}^{i} \cdot \mathbf{e}_{\varphi}$ and $S_{+}^{i}:=\frac{p_{+}^{i}}{\left(\rho_{+}^{i}\right)^{\gamma}}$ for $i=1,2$ (here we used Lemma 3 and the fact that if $\left(\rho_{+}^{i}, \mathbf{u}_{+}^{i}, p_{+}^{i}\right)$ are $C^{0}\left(\overline{\mathcal{N}_{f}^{+}}\right) \cap C^{1}\left(\mathcal{N}_{f}^{+}\right)$ solutions of Problem 1, then $(\rho, \mathbf{u}, p)=\left(\rho_{+}^{i}, \mathbf{u}_{+}^{i}, p_{+}^{i}\right)$ satisfy $B=B_{0}$ in $\left.\overline{\mathcal{N}_{f^{i}}^{+}}\right)$.

Take $\underline{\sigma}_{2}=\underline{\sigma}_{2}^{(1)}$ so that 1) and 2) hold. Let $\left(\Phi^{i} \mathbf{e}_{\varphi}, L^{i}, S^{i}\right)$ for $i=1,2$ be $\left(\Phi \mathbf{e}_{\varphi}, L, S\right)$ given by the definitions of $\Phi, L$ and $S$ for $(\rho, \mathbf{u}, p)=\left(\rho_{+}^{i}, \mathbf{u}_{+}^{i}, p_{+}^{i}\right)$ for $i=1,2$. Then since $\left(f^{i}, \rho_{+}^{i}, \mathbf{u}_{+}^{i}, p_{+}^{i}\right)$ for $i=1,2$ are solutions of Problem 1 , $\left(f^{i}, \Phi^{i} \mathbf{e}_{\varphi}, L^{i}, S^{i}\right)$ for $i=1,2$ are solutions of Problem 2. Furthermore, since $\left(f^{i}, \rho_{+}^{i}, \mathbf{u}_{+}^{i}, p_{+}^{i}\right)$ for $i=1,2$ satisfy (2.56), $\left(f^{i}, \Phi^{i} \mathbf{e}_{\varphi}, L^{i}, S^{i}\right)$ for $i=1,2$ satisfy (2.55) with $C$ replaced by $C_{1}$ for some positive constant $C_{1}$ depending on the data (here we used (2.60)). Take $\underline{\sigma}_{2}=\min \left(\underline{\sigma}_{2}^{(1)}, \frac{C \sigma_{1}}{C_{1}}, \sigma_{1}\right)$ where $C$ is $C$ in (2.55) so that $\left(\rho_{-}, \mathbf{u}_{-}, p_{-}\right)$and $p_{e x}$ satisfy (2.19) and (2.20), respectively, for $\sigma \in\left(0, \sigma_{1}\right]$ and 
( $f^{i}, \Phi^{i} \mathbf{e}_{\varphi}, L^{i}, S^{i}$ ) for $i=1,2$ satisfy (2.55) for $\sigma \in\left(0, \sigma_{1}\right]$. Then by Theorem 1 , $\left(f^{1}, \Phi^{1} \mathbf{e}_{\varphi}, L^{1}, S^{1}\right)=\left(f^{2}, \Phi^{2} \mathbf{e}_{\varphi}, L^{2}, S^{2}\right)$. This implies

$$
\rho_{+}^{1}\left(u_{+, r}^{1} \mathbf{e}_{r}+u_{+, \theta}^{1} \mathbf{e}_{\theta}\right)=\rho_{+}^{2}\left(u_{+, r}^{2} \mathbf{e}_{r}+u_{+, \theta}^{2} \mathbf{e}_{\theta}\right) \text { and } u_{+, \varphi}^{1} \mathbf{e}_{\varphi}=u_{+, \varphi}^{2} \mathbf{e}_{\varphi} .
$$

By these relations, $S^{1}=S^{2}$ and 2), we have that $\rho_{+}^{1}=\rho_{+}^{2}$. With this relation, we can conclude that $\left(\rho_{+}^{1}, \mathbf{u}_{+}^{1}, p_{+}^{1}\right)=\left(\rho_{+}^{2}, \mathbf{u}_{+}^{2}, p_{+}^{2}\right)$. Let $\sigma_{2}=\min \left(\bar{\sigma}_{2}, \underline{\sigma}_{2}\right)$. This finishes the proof.

The rest of this paper is devoted to proving Theorem 1. For convenience, we describe our main process of proving Theorem 1 below.

To describe our process of proving Theorem 1, we define some terminologies. Let $f:\left[0, \theta_{1}\right] \rightarrow \mathbb{R}$ be a function representing an axisymmetric shock location $\Gamma_{f}$. Decompose $f$ into $f(\theta)=f(0)+f_{s}(\theta)$. Then by $f_{s}(0)=0, f_{s}$ is uniquely determined by $f_{s}^{\prime}$. We call $f(0)$ and $f_{s}^{\prime}$ the initial shock position and the shape of a shock location, respectively.

Using these terminologies, our process of proving Theorem 1 is described as follows:

1. For given an incoming supersonic solution, an exit pressure and a shape of a shock location $\left(\rho_{-}, \mathbf{u}_{-}, p_{-}, p_{e x}, f_{s}^{\prime}\right)$ in a small perturbation of $\left(\rho_{0}^{-}, u_{0}^{-} \mathbf{e}_{r}\right.$, $\left.p_{0}^{-}, p_{c}, 0\right)$, show that there exists a pair of an initial shock position $f(0)$ and a subsonic solution ( $\left.\Phi \mathbf{e}_{\varphi}, L, S\right)$ of (2.43)-(2.45) satisfying all the conditions in Problem 2 except (2.47), and that this solution is unique in the class of functions in a small perturbation of $\left(r_{s}, \Phi_{0}^{+} \mathbf{e}_{\varphi}, L_{0}^{+}, S_{0}^{+}\right)$.

2. For given an incoming supersonic solution and an exit pressure as in Step 1 or in a much small perturbation of $\left(\rho_{0}^{-}, u_{0}^{-} \mathbf{e}_{r}, p_{0}^{-}, p_{c}\right)$ if necessary, show that there exists $f_{S}^{\prime}$ in a small perturbation of 0 as in Step 1 such that $\left(f(0), \Phi \mathbf{e}_{\varphi}, L, S\right)$ determined by $\left(\rho_{-}, \mathbf{u}_{-}, p_{-}, p_{e x}, f_{s}^{\prime}\right)$ in Step 1 satisfies (2.47), and that for given $\left(\rho_{-}, \mathbf{u}_{-}, p_{-}, p_{e x}\right)$ in a small perturbation of $\left(\rho_{0}^{-}, u_{0}^{-} \mathbf{e}_{r}, p_{0}^{-}, p_{c}\right)$, a solution $\left(f, \Phi \mathbf{e}_{\varphi}, L, S\right)$ of Problem 2 is unique in the class of functions in a small perturbation of $\left(r_{s}, \Phi_{0}^{+} \mathbf{e}_{\varphi}, L_{0}^{+}, S_{0}^{+}\right)$.

Once Step 1 and Step 2 are done, then $f=f(0)+f_{s}$ and $\left(\Phi \mathbf{e}_{\varphi}, L, S\right)$ obtained through Step 1 and Step 2 satisfies all the conditions in Problem 2. Thus, Theorem 1 is proved if Step 1 and Step 2 are done. We will deal with Step 1 and Step 2 in Sections 3 and 4, respectively.

Note that the fact that for transonic shock solutions of the full Euler system, an initial shock position and a shape of a shock location are determined in different mechanisms (an initial shock position is determined by the solvability condition related to the mass conservation law and a shape of a shock location is determined by the R-H conditions) was pointed out in [24] and the authors in [10,18,20,21,23,24] proved the stability of transonic shock solutions of the full Euler system using iteration schemes based on this fact. In this paper, we also prove the stability of transonic shock solutions of the full Euler system using a scheme based on this fact, but we do this using a different scheme. In our scheme, a non-local elliptic equation appearing in $[10,18,20,21,23,24]$ does not appear. 


\section{Pseudo Free Boundary Problem}

As a first step to prove Theorem 1, we will solve the Pseudo Free Boundary Problem below. This problem naturally arises from the requirement that a subsonic solution in Problem 2 must satisfy (2.54). From the linearized equation of (2.53), it is seen that an iteration scheme for a fixed boundary problem does not give a subsonic solution satisfying (2.54) in general. From this, we have that an iteration scheme for a fixed boundary problem is not a proper scheme to find a subsonic solution in Problem 2. To find a subsonic solution satisfying (2.54), an iteration scheme that gives a proper $(\rho, \mathbf{u}, p)$ on $\Gamma_{e x}$ is necessary. To find a subsonic solution satisfying (2.54), we let $f(0)$ be an unknown to be determined simultaneously with a subsonic solution. Using this variable, we adjust the value of a subsonic solution so that this solution can satisfy (2.54). The main ingredient for this argument to hold is the monotonicity of the entropy of the downstream subsonic solution of a radial transonic shock solution on a shock location with respect to the shock location. This will be seen in the proof of Proposition 2.

Problem 3. (Pseudo Free Boundary Problem) Given an axisymmetric supersonic solution $\left(\rho_{-}, \mathbf{u}_{-}, p_{-}\right)$of (2.1) and exit pressure $p_{\text {ex }}$ as in Problem 1 and a shape of a shock location $f_{s}^{\prime} \in C_{\left(-\alpha,\left\{\theta=\theta_{1}\right\}\right)}^{1, \alpha}\left(\left(0, \theta_{1}\right)\right)$ satisfying $f_{s}^{\prime}(\theta)=0$ at $\theta=0, \theta_{1}$ and

$$
\left\|f_{S}^{\prime}\right\|_{1, \alpha,\left(0, \theta_{1}\right)}^{\left(-\alpha,\left\{\theta=\theta_{1}\right\}\right)} \leq \sigma
$$

for a sufficiently small $\sigma>0$, find an initial shock position $f(0)$ and a corresponding subsonic solution $\left(\Phi \mathbf{e}_{\varphi}, L, S\right)$ of (2.43)-(2.45) satisfying

$$
\begin{aligned}
& \left(\frac{1}{\rho} \nabla \times\left(\Phi \mathbf{e}_{\varphi}\right) \cdot \mathbf{e}_{r}\right) \nabla \times\left(\frac{1}{\rho} \nabla \times\left(\Phi \mathbf{e}_{\varphi}\right)\right) \\
& \quad=\left(\frac{L}{2 \pi r \sin \theta} \nabla \times\left(\frac{L}{2 \pi r \sin \theta} \mathbf{e}_{\varphi}\right) \cdot \mathbf{e}_{r}+\frac{\rho^{\gamma-1}}{\gamma-1} \frac{\partial_{\theta} S}{r}\right) \mathbf{e}_{\varphi} \text { in } \mathcal{N}_{f(0)+f_{s}}^{+}, \\
& \Phi \mathbf{e}_{\varphi}=\Phi_{-} \mathbf{e}_{\varphi} \text { on } \Gamma_{f(0)+f_{s}}, \\
& \Phi \mathbf{e}_{\varphi}=\frac{r_{0} \Phi_{-}\left(r_{0}, \theta_{1}\right)}{r} \mathbf{e}_{\varphi} \text { on } \Gamma_{w, f(0)+f_{s}}^{+}, \\
& S \rho^{\gamma}=p_{e x} \text { on } \Gamma_{e x}, \\
& \lim _{\theta \rightarrow \theta_{1}}\left(\Phi \mathbf{e}_{\varphi}\right)\left(r_{1}, \theta\right)=\lim _{r \rightarrow r_{1}}\left(\Phi \mathbf{e}_{\varphi}\right)\left(r, \theta_{1}\right),
\end{aligned}
$$

where $\rho=\varrho\left(\nabla \times\left(\Phi \mathbf{e}_{\varphi}\right), \frac{L}{2 \pi r \sin \theta} \mathbf{e}_{\varphi}, S\right)$, and

$$
\begin{aligned}
& \nabla \times\left(\Phi \mathbf{e}_{\varphi}\right) \cdot \nabla L=0 \text { in } \mathcal{N}_{f(0)+f_{s}}^{+}, \\
& L=L_{-} \text {on } \Gamma_{f(0)+f_{s}}, \\
& \nabla \times\left(\Phi \mathbf{e}_{\varphi}\right) \cdot \nabla S=0 \text { in } \mathcal{N}_{f(0)+f_{s}}^{+}, \\
& S=g\left(\left(\frac{\mathbf{u}_{-} \cdot \boldsymbol{v}_{f}}{c_{-}}\right)^{2}\right) S_{-} \text {on } \Gamma_{f(0)+f_{s}}
\end{aligned}
$$


where $f_{s}$ denotes $\int_{0}^{\theta} f_{s}^{\prime}$.

Hereafter, we denote a set of functions in $C_{\left(-\alpha,\left\{\theta=\theta_{1}\right\}\right)}^{1, \alpha}\left(\left(0, \theta_{1}\right)\right)$ having 0 value at $\theta=0, \theta_{1}$ by $C_{\left(-\alpha,\left\{\theta=\theta_{1}\right\}\right), 0}^{1, \alpha}\left(\left(0, \theta_{1}\right)\right)$. Hereafter, $f_{s}$ denotes $\int_{0}^{\theta} f_{s}^{\prime}$.

Our result of Problem 3 is given as follows:

Proposition 2. Let $\alpha \in\left(\frac{2}{3}, 1\right)$. There exists a positive constant $\sigma_{3}$ depending on the data such that if $\sigma \in\left(0, \sigma_{3}\right]$, then Problem 3 has a solution $\left(f(0), \Phi \mathbf{e}_{\varphi}, L, S\right)$ satisfying

$$
\begin{aligned}
& \left|f(0)-r_{s}\right|+\left\|\nabla \times\left(\left(\Phi-\Phi_{0}^{+}\right) \mathbf{e}_{\varphi}\right)\right\|_{1, \alpha, \mathcal{N}_{f(0)+f_{s}}^{+}}^{\left(-\alpha, \Gamma_{w}^{+}\right)} \\
& \quad+\left\|\frac{L}{2 \pi r \sin \theta} \mathbf{e}_{\varphi}\right\|_{1, \alpha, \mathcal{N}_{f(0)+f_{s}}^{+}}^{\left(-\alpha, \Gamma_{+}^{+}\right)}+\left\|S-S_{0}^{+}\right\|_{1, \alpha, \mathcal{N}_{f(0)+f_{s}}^{+}}^{\left(-\alpha, \Gamma_{+}^{+}\right)} \leq C \sigma
\end{aligned}
$$

where $C$ is a positive constant depending on the data. Furthermore, this solution is unique in the class of functions satisfying (3.2).

We will prove Proposition 2 using a fixed point argument. To do this, we linearize (A) with respect to (A) satisfied by $\left(\Phi \mathbf{e}_{\varphi}, L, S\right)=\left(\Phi_{0}^{+} \mathbf{e}_{\varphi}, L_{0}^{+}, S_{0}^{+}\right)$and reformulate (B) in terms of $\left(\Psi \mathbf{e}_{\varphi}, A, T\right)$ where $(\Psi, A, T):=\left(\Phi-\Phi_{0}^{+}, L, S-S_{0}^{+}\right)$.

\subsection{Linearization and reformulation of $(A)$ and $(B)$}

We linearize (A) with respect to (A) satisfied by $\left(\Phi_{0}^{+} \mathbf{e}_{\varphi}, L_{0}^{+}, S_{0}^{+}\right)$. Since $\rho$ in the first and fourth equation of $(\mathrm{A})$ is given using an implicit relation, to obtain the linearized equations of (A), we first linearize $\rho$ with respect to $\rho_{0}^{+}$.

Lemma 4. Suppose that $f: \Lambda \rightarrow \mathbb{R}$ is an axisymmetric function in $C_{(-1-\alpha, \partial \Lambda)}^{2, \alpha}$ ( $\Lambda$ ) satisfying

$$
\left\|f-r_{s}\right\|_{2, \alpha, \Lambda}^{(-1-\alpha, \partial \Lambda)} \leq \delta_{1} .
$$

Also, suppose that $\Psi \mathbf{e}_{\varphi}: \mathcal{N}_{f}^{+} \rightarrow \mathbb{R}^{3}, A: \mathcal{N}_{f}^{+} \rightarrow \mathbb{R}$ and $T: \mathcal{N}_{f}^{+} \rightarrow \mathbb{R}$ are axisymmetric functions in $C_{\left(-1-\alpha, \Gamma_{w}^{+}\right)}^{2, \alpha}\left(\mathcal{N}_{f}^{+}\right), C_{\left(-\alpha, \Gamma_{w}^{+}\right)}^{1, \alpha}\left(\mathcal{N}_{f}^{+}\right)$and $C_{\left(-\alpha, \Gamma_{w}^{+}\right)}^{1, \alpha}\left(\mathcal{N}_{f}^{+}\right)$, respectively, and satisfy

$$
\left\|\nabla \times\left(\Psi \mathbf{e}_{\varphi}\right)\right\|_{1, \alpha, \mathcal{N}_{f}^{+}}^{\left(-\alpha, \Gamma_{w}^{+}\right)}+\left\|\frac{A}{2 \pi r \sin \theta} \mathbf{e}_{\varphi}\right\|_{1, \alpha, \mathcal{N}_{f}^{+}}^{\left(-\alpha, \Gamma_{+}^{+}\right)}+\|T\|_{1, \alpha, \mathcal{N}_{f}^{+}}^{\left(-\alpha, \Gamma^{+}\right)} \leq \delta_{2} .
$$

Let $\rho=\varrho\left(\nabla \times\left(\Phi \mathbf{e}_{\varphi}\right), \frac{L}{2 \pi r \sin \theta} \mathbf{e}_{\varphi}, S\right)$. There holds

$$
\begin{aligned}
\rho & -\rho_{0}^{+} \\
& =\frac{\nabla \times\left(\Phi_{0}^{+} \mathbf{e}_{\varphi}\right)}{\rho_{0}^{+}\left(u_{0}^{+2}-c_{0}^{+2}\right)} \cdot \nabla \times\left(\Psi \mathbf{e}_{\varphi}\right)+\frac{\gamma \rho_{0}^{+\gamma}}{(\gamma-1)\left(u_{0}^{+2}-c_{0}^{+2}\right)} T \\
& +g_{1}\left(\Psi \mathbf{e}_{\varphi}, A, T\right),
\end{aligned}
$$


where

$$
\begin{aligned}
g_{1}( & \left.\Psi \mathbf{e}_{\varphi}, A, T\right) \\
= & \frac{1}{\rho_{0}^{+}\left(u_{0}^{+2}-c_{0}^{+2}\right)}\left(\int _ { 0 } ^ { 1 } \left(\left(2 \rho_{0}^{+} B_{0}-\frac{\gamma(\gamma+1)}{\gamma-1} S_{0}^{+} \rho_{0}^{+\gamma}\right)\right.\right. \\
& \left.-\left(2\left(t \rho+(1-t) \rho_{0}^{+}\right) B_{0}-\frac{\gamma(\gamma+1)}{\gamma-1}\left(S_{0}^{+}+T\right)\left(t \rho+(1-t) \rho_{0}^{+}\right)^{\gamma}\right)\right) d t\left(\rho-\rho_{0}^{+}\right) \\
& +\int_{0}^{1}\left(-\nabla \times\left(\Phi_{0}^{+} \mathbf{e}_{\varphi}\right)+\left(t \nabla \times\left(\left(\Phi_{0}^{+}+\Psi\right) \mathbf{e}_{\varphi}\right)+(1-t) \nabla \times\left(\Phi_{0}^{+} \mathbf{e}_{\varphi}\right)\right)\right) d t \\
& \left.\cdot \nabla \times\left(\Psi \mathbf{e}_{\varphi}\right)+\int_{0}^{1} \frac{\rho_{0}^{+2} t A}{2 \pi r \sin \theta} \mathbf{e}_{\varphi} d t \cdot \frac{A}{2 \pi r \sin \theta} \mathbf{e}_{\varphi}\right) .
\end{aligned}
$$

$g_{1}\left(\Psi \mathbf{e}_{\varphi}, A, T\right)$ satisfies

$$
\begin{aligned}
& \left\|g_{1}\left(\Psi \mathbf{e}_{\varphi}, A, T\right)\right\|_{1, \alpha, \mathcal{N}_{f}^{+}}^{\left(-\alpha, \Gamma_{w}^{+}\right)} \\
& \leq C\left(\left\|\nabla \times\left(\Psi \mathbf{e}_{\varphi}\right)\right\|_{1, \alpha, \mathcal{N}_{f}^{+}}^{\left(-\alpha, \Gamma_{w}^{+}\right)}+\left\|\frac{A}{2 \pi r \sin \theta} \mathbf{e}_{\varphi}\right\|_{1, \alpha, \mathcal{N}_{f}^{+}}^{\left(-\alpha, \Gamma_{w}^{+}\right)}\right. \\
& \left.\quad+\|T\|_{1, \alpha, \mathcal{N}_{f}^{+}}^{\left(-\alpha, \Gamma_{w}^{+}\right)}\right)^{2}
\end{aligned}
$$

where $C$ is a positive constant depending on $\left(\rho_{0}^{+}, u_{0}^{+}, p_{0}^{+}\right), \gamma, r_{s}, r_{1}, \alpha, \delta_{1}$ and $\delta_{2}$.

Proof. 1. By the definition of $\varrho, \rho=\varrho\left(\nabla \times\left(\Phi \mathbf{e}_{\varphi}\right), \frac{L}{2 \pi r \sin \theta} \mathbf{e}_{\varphi}, S\right)$ satisfies

$$
b\left(\rho, \nabla \times\left(\Phi \mathbf{e}_{\varphi}\right), \frac{L}{2 \pi r \sin \theta} \mathbf{e}_{\varphi}, S, B_{0}\right)=0,
$$

where $b$ is a function defined in (2.40). Subtract this equation from $b\left(\rho_{0}^{+}, \nabla \times\right.$ $\left.\left(\Phi_{0}^{+} \mathbf{e}_{\varphi}\right), 0, S_{0}^{+}, B_{0}\right)=0$ obtained from the third equation of (2.14). And then linearize the resultant equation. Then we obtain (3.5).

2. With the fact that $\left(\rho_{0}^{+}, u_{0}^{+} \mathbf{e}_{r}, p_{0}^{+}\right) \in\left(C^{\infty}\left(\overline{\mathcal{N}_{f}^{+}}\right)\right)^{3}$ and (3.4), estimate $\rho-\rho_{0}^{+}$ in $C_{\left(-\alpha, \Gamma_{w}^{+}\right)}^{1, \alpha}\left(\mathcal{N}_{f}^{+}\right)$in the way that we estimated $\rho_{+}-\rho_{0}^{+}$in the proof of Theorem 2. Then we have

$$
\begin{aligned}
\| \rho & -\rho_{0}^{+} \|_{1, \alpha, \mathcal{N}_{f}^{+}}^{\left(-\alpha, \Gamma_{w}^{+}\right)} \\
\leq & C\left(\left\|\nabla \times\left(\Psi \mathbf{e}_{\varphi}\right)\right\|_{1, \alpha, \mathcal{N}_{f}^{+}}^{\left(-\alpha, \Gamma_{w}^{+}\right)}+\left\|\frac{A}{2 \pi r \sin \theta} \mathbf{e}_{\varphi}\right\|_{1, \alpha, \mathcal{N}_{f}^{+}}^{\left(-\alpha, \Gamma^{+}\right)}\right. \\
& \left.+\|T\|_{1, \alpha, \mathcal{N}_{f}^{+}}^{\left(-\alpha, \Gamma_{+}^{+}\right)}\right)
\end{aligned}
$$

where $C$ is a positive constant depending on $\left(\rho_{0}^{+}, u_{0}^{+}, p_{0}^{+}\right), \gamma, r_{s}, r_{1}, \alpha, \delta_{1}$ and $\delta_{2}$. With this estimate, the fact that $\left(\rho_{0}^{+}, u_{0}^{+} \mathbf{e}_{r}, p_{0}^{+}\right) \in\left(C^{\infty}\left(\overline{\mathcal{N}_{f}^{+}}\right)\right)^{3}$ and (3.4), estimate (3.6) in $C_{\left(-\alpha, \Gamma_{w}^{+}\right)}^{1, \alpha}\left(\mathcal{N}_{f}^{+}\right)$. Then we obtain the desired result. 
Then we linearize $(\mathrm{A})$.

Subtract (2.43) satisfied by $\left(\Phi \mathbf{e}_{\varphi}, L, S\right)=\left(\Phi_{0}^{+} \mathbf{e}_{\varphi}, L_{0}^{+}, S_{0}^{+}\right)$in $\mathcal{N}_{f}^{+}$from (2.43) in $\mathcal{N}_{f}^{+}$and then linearize the resultant equation (in this process, we express $\rho-\rho_{0}^{+}$ using (3.5)). Then we obtain

$$
\begin{aligned}
\nabla & \times\left(\frac{1}{\rho_{0}^{+}}\left(1+\frac{u_{0}^{+} \mathbf{e}_{r} \otimes u_{0}^{+} \mathbf{e}_{r}}{c_{0}^{+2}-u_{0}^{+2}}\right) \nabla \times\left(\Psi \mathbf{e}_{\varphi}\right)\right) \\
& =\frac{\rho_{0}^{+\gamma-1}}{(\gamma-1) u_{0}^{+}}\left(1+\frac{\gamma u_{0}^{+2}}{c_{0}^{+2}-u_{0}^{+2}}\right) \frac{\partial_{\theta} T}{r} \mathbf{e}_{\varphi}+\boldsymbol{F}_{1}\left(\Psi \mathbf{e}_{\varphi}, A, T\right) \text { in } \mathcal{N}_{f}^{+} \\
& \left(=: \boldsymbol{F}_{2}\left(\Psi \mathbf{e}_{\varphi}, A, T\right)\right)
\end{aligned}
$$

where

$$
\begin{aligned}
\boldsymbol{F}_{1}\left(\Psi \mathbf{e}_{\varphi}, A, T\right) \\
=\nabla \times\left(\frac{1}{\rho_{0}^{+}} g_{1} \nabla \times\left(\Phi_{0}^{+} \mathbf{e}_{\varphi}\right)-\frac{1}{\rho \rho_{0}^{+2}} g_{2}^{2} \nabla \times\left(\left(\Phi_{0}^{+}+\Psi\right) \mathbf{e}_{\varphi}\right)+\frac{1}{\rho_{0}^{+2}} g_{2} \nabla \times\left(\Psi \mathbf{e}_{\varphi}\right)\right) \\
\quad-\frac{1}{u_{0}^{+}}\left(-\frac{1}{\rho \rho_{0}^{+}} g_{2} \nabla \times\left(\left(\Phi_{0}^{+}+\Psi\right) \mathbf{e}_{\varphi}\right) \cdot \mathbf{e}_{r}+\frac{1}{\rho_{0}^{+}} \nabla \times\left(\Psi \mathbf{e}_{\varphi}\right) \cdot \mathbf{e}_{r}\right) \\
\quad \nabla \times\left(-\frac{1}{\rho \rho_{0}^{+}} g_{2} \nabla \times\left(\left(\Phi_{0}^{+}+\Psi\right) \mathbf{e}_{\varphi}\right)+\frac{1}{\rho_{0}^{+}} \nabla \times\left(\Psi \mathbf{e}_{\varphi}\right)\right) \\
+\frac{1}{u_{0}^{+}}\left(\int_{0}^{1}\left(t \rho+(1-t) \rho_{0}^{+}\right)^{\gamma-2} \mathrm{~d} t g_{2} \frac{\partial_{\theta} T}{r}\right) \mathbf{e}_{\varphi} \\
+\frac{A}{u_{0}^{+} 2 \pi r \sin \theta}\left(\nabla \times\left(\frac{A}{2 \pi r \sin \theta} \mathbf{e}_{\varphi}\right) \cdot \mathbf{e}_{r}\right) \mathbf{e}_{\varphi},
\end{aligned}
$$

where $g_{1}$ and $g_{2}$ are $g_{1}\left(\Psi \mathbf{e}_{\varphi}, A, T\right)$ given in (3.6) and the right hand side of (3.5), respectively.

By the definition of $V_{0}^{ \pm}$and the first equations of (2.10) and (2.14), $V_{0}^{+}=V_{0}^{-}$on $\Gamma_{f}$. In the vector potential form, this is written as $\Phi_{0}^{+} \mathbf{e}_{\varphi}$ $=\Phi_{0}^{-} \mathbf{e}_{\varphi}$ on $\Gamma_{f}$. Subtract this equation from (2.46). Then we obtain

$$
\Psi \mathbf{e}_{\varphi}=\left(\Phi_{-}-\Phi_{0}^{-}\right) \mathbf{e}_{\varphi} \quad \text { on } \quad \Gamma_{f}
$$

By the definition of $V_{0}^{ \pm}$and the first equations of (2.10) and (2.14), $V_{0}^{+}=$ $V_{0}^{-}\left(r_{0}, \theta_{1}\right)$ on $\Gamma_{w}^{+}$. In the vector potential form, this is written as $\Phi_{0}^{+} \mathbf{e}_{\varphi}=\frac{r_{0} \Phi_{0}^{-}\left(r_{0}, \theta_{1}\right) \mathbf{e}_{\varphi}}{r}$ on $\Gamma_{w}^{+}$. By subtracting this equation from (2.52), we obtain

$$
\Psi \mathbf{e}_{\varphi}=\frac{r_{0}\left(\Phi_{-}-\Phi_{0}^{-}\right)\left(r_{0}, \theta_{1}\right)}{r} \mathbf{e}_{\varphi} \text { on } \Gamma_{w}^{+}
$$

Rewrite (2.53) as $\rho=\left(\frac{p_{e x}}{S}\right)^{\frac{1}{\gamma}}$ on $\Gamma_{e x}$. Subtract this equation from $\rho_{0}^{+}=\left(\frac{p_{c}}{S_{0}^{+}}\right)^{\frac{1}{\gamma}}$ on $\Gamma_{e x}$. And then express $\rho-\rho_{0}^{+}$using (3.5) and linearize $\left(\frac{p_{e x}}{S}\right)^{\frac{1}{\gamma}}-\left(\frac{p_{c}}{S_{0}^{+}}\right)^{\frac{1}{\gamma}}$. Multiply 
$\frac{u_{0}^{+2}-c_{0}^{+2}}{u_{0}^{+}} r \sin \theta$ and then integrate the resultant equation from 0 to $\theta$. After that, divide $\sin \theta$ and multiply $\mathbf{e}_{\varphi}$ on both-hand sides of the equation. Then we obtain

$$
\begin{aligned}
\Psi \mathbf{e}_{\varphi}=( & \frac{1}{r \sin \theta} \int_{0}^{\theta}\left(\mathfrak{f}_{0}\left(T, p_{e x}\right)-\frac{\rho_{0}^{+}\left((\gamma-1) u_{0}^{+2}+c_{0}^{+2}\right)}{\gamma(\gamma-1) u_{0}^{+} S_{0}^{+}} T\right. \\
& \left.\left.+\mathfrak{f}_{1}\left(\Psi \mathbf{e}_{\varphi}, A, T\right)\right) r^{2} \sin \xi \mathrm{d} \xi\right) \mathbf{e}_{\varphi} \text { on } \Gamma_{e x},
\end{aligned}
$$

where

$$
\mathfrak{f}_{0}\left(T, p_{e x}\right)=\frac{u_{0}^{+^{2}}-c_{0}^{+2}}{u_{0}^{+}}\left(\frac{1}{S_{0}^{+}+T}\right)^{\frac{1}{\gamma}}\left(p_{e x}^{\frac{1}{\gamma}}-p_{c}^{\frac{1}{\gamma}}\right)
$$

and

$$
\begin{aligned}
\mathfrak{f}_{1}\left(\Psi \mathbf{e}_{\varphi}, A, T\right) & =-\frac{p_{c}^{\frac{1}{\gamma}}\left(u_{0}^{+2}-c_{0}^{+2}\right)}{\gamma u_{0}^{+}} \int_{0}^{1}\left(\left(\frac{1}{t S+(1-t) S_{0}^{+}}\right)^{\frac{1}{\gamma}+1}-\left(\frac{1}{S_{0}^{+}}\right)^{\frac{1}{\gamma}+1}\right) \mathrm{d} t T \\
& -\frac{u_{0}^{+2}-c_{0}^{+2}}{u_{0}^{+}} g_{1} .
\end{aligned}
$$

By the definition of $V_{0}^{ \pm}$and the first equation of (2.14), $\lim _{\theta \rightarrow \theta_{1}} V_{0}^{+}\left(r_{1}, \theta\right)=$ $\lim _{r \rightarrow r_{1}} V_{0}^{+}\left(r, \theta_{1}\right)$. In the vector potential form, this is written as $\lim _{\theta \rightarrow \theta_{1}}\left(\Phi_{0}^{+} \mathbf{e}_{\varphi}\right)$ $\left(r\left(r_{1}, \theta\right)=\lim _{r \rightarrow r_{1}}\left(\Phi_{0}^{+} \mathbf{e}_{\varphi}\right), \theta_{1}\right)$. Subtract this equation from (2.54). Then we obtain

$$
\lim _{\theta \rightarrow \theta_{1}}\left(\Psi \mathbf{e}_{\varphi}\right)\left(r_{1}, \theta\right)=\lim _{r \rightarrow r_{1}}\left(\Psi \mathbf{e}_{\varphi}\right)\left(r, \theta_{1}\right) .
$$

Express this relation using (3.12) and (3.13). Then we have

$$
\begin{aligned}
& \frac{1}{r_{1} \sin \theta_{1}} \int_{0}^{\theta_{1}}\left(\mathfrak{f}_{0}\left(T, p_{e x}\right)-\frac{\rho_{0}^{+}\left((\gamma-1) u_{0}^{+2}+c_{0}^{+2}\right)}{\gamma(\gamma-1) u_{0}^{+} S_{0}^{+}} T\right. \\
& \left.\quad+\mathfrak{f}_{1}\left(\Psi \mathbf{e}_{\varphi}, A, T\right)\right)\left.\right|_{r=r_{1}} r_{1}^{2} \sin \xi \mathrm{d} \xi=\frac{r_{0}\left(\Phi_{-}-\Phi_{0}^{-}\right)\left(r_{0}, \theta_{1}\right)}{r_{1}} .
\end{aligned}
$$

Combining (3.9), (3.11), (3.12), (3.13) and (3.16), we have the following linearized equations of (A):

$$
\begin{aligned}
\nabla \times & \left(\frac{1}{\rho_{0}^{+}}\left(1+\frac{u_{0}^{+} \mathbf{e}_{r} \otimes u_{0}^{+} \mathbf{e}_{r}}{c_{0}^{+^{2}}-u_{0}^{+2}}\right) \nabla \times\left(\Psi \mathbf{e}_{\varphi}\right)\right) \\
& =\frac{\rho_{0}^{+\gamma-1}}{(\gamma-1) u_{0}^{+}}\left(1+\frac{\gamma u_{0}^{+2}}{c_{0}^{+2}-u_{0}^{+2}}\right) \frac{\partial_{\theta} T}{r} \mathbf{e}_{\varphi}+\boldsymbol{F}_{1}\left(\Psi \mathbf{e}_{\varphi}, A, T\right) \text { in } \mathcal{N}_{f(0)+f_{s}}^{+},
\end{aligned}
$$




$$
\begin{aligned}
& \Psi \mathbf{e}_{\varphi}=\left(\Phi_{-}-\Phi_{0}^{-}\right) \mathbf{e}_{\varphi} \text { on } \Gamma_{f(0)+f_{s}}, \\
& \Psi \mathbf{e}_{\varphi}=\frac{r_{0}\left(\Phi_{-}-\Phi_{0}^{-}\right)\left(r_{0}, \theta_{1}\right)}{r} \mathbf{e}_{\varphi} \text { on } \Gamma_{w, f(0)+f_{s}}^{+}, \\
& \Psi \mathbf{e}_{\varphi}=\left(\frac { 1 } { r \operatorname { s i n } \theta } \int _ { 0 } ^ { \theta } \left(\mathfrak{f}_{0}\left(T, p_{e x}\right)-\frac{\rho_{0}^{+}\left((\gamma-1) u_{0}^{+2}+c_{0}^{+2}\right)}{\gamma(\gamma-1) u_{0}^{+} S_{0}^{+}} T\right.\right. \\
& \left.\left.+\mathfrak{f}_{1}\left(\Psi \mathbf{e}_{\varphi}, A, T\right)\right) r^{2} \sin \xi \mathrm{d} \xi\right) \mathbf{e}_{\varphi} \text { on } \Gamma_{e x}, \\
& \frac{1}{r_{1} \sin \theta_{1}} \int_{0}^{\theta_{1}}\left(\mathfrak{f}_{0}\left(T, p_{e x}\right)-\frac{\rho_{0}^{+}\left((\gamma-1) u_{0}^{+2}+c_{0}^{+2}\right)}{\gamma(\gamma-1) u_{0}^{+} S_{0}^{+}} T\right. \\
& \left.\quad+\mathfrak{f}_{1}\left(\Psi \mathbf{e}_{\varphi}, A, T\right)\right)\left.\right|_{r=r_{1}} r_{1}^{2} \sin \xi \mathrm{d} \xi=\frac{r_{0}\left(\Phi_{-}-\Phi_{0}^{-}\right)\left(r_{0}, \theta_{1}\right)}{r_{1}} .
\end{aligned}
$$

For later use, we present the following estimates of $\boldsymbol{F}_{1}\left(\Psi \mathbf{e}_{\varphi}, A, T\right)$ and $\mathfrak{f}_{1}\left(\Psi \mathbf{e}_{\varphi}\right.$, $A, T)$.

Lemma 5. Let $\delta_{4}$ be a positive constant $\leq \delta_{2}$ such that for $f$ as in Lemma 4, if $\left(\Psi \mathbf{e}_{\varphi}, A, T\right)$ satisfies

$$
\left\|\nabla \times\left(\Psi \mathbf{e}_{\varphi}\right)\right\|_{1, \alpha, \mathcal{N}_{f}^{+}}^{\left(-\alpha, \Gamma_{w}^{+}\right)}+\left\|\frac{A}{2 \pi r \sin \theta} \mathbf{e}_{\varphi}\right\|_{1, \alpha, \mathcal{N}_{f}^{+}}^{\left(-\alpha, \Gamma_{w}^{+}\right)}+\|T\|_{1, \alpha, \mathcal{N}_{f}^{+}}^{\left(-\alpha, \Gamma_{w}^{+}\right)} \leq \delta_{4},
$$

then

$$
\sup _{\mathcal{N}_{f}^{+}}\left|\frac{1}{\varrho\left(\nabla \times\left(\Phi \mathbf{e}_{\varphi}\right), \frac{L}{2 \pi r \sin \theta} \mathbf{e}_{\varphi}, S\right)}\right| \leq C \quad \text { and } \quad \sup _{\mathcal{N}_{f}^{+}}\left|\frac{1}{S}\right| \leq C
$$

where $C$ s are positive constants depending on $\left(\rho_{0}^{+}, u_{0}^{+}, p_{0}^{+}\right), \gamma, r_{s}, r_{1}$ and $\delta_{4}$. Suppose that $f$ is as in Lemma 4 . Also, suppose that $\left(\Psi \mathbf{e}_{\varphi}, A, T\right)$ are as in Lemma 4 and satisfy (3.17). Then there hold

$$
\begin{aligned}
& \left\|\boldsymbol{F}_{1}\left(\Psi \mathbf{e}_{\varphi}, A, T\right)\right\|_{\alpha, \mathcal{N}_{f}^{+}}^{\left(1-\alpha, \Gamma_{w}^{+}\right)} \\
& \quad \leq C\left(\left\|\Psi \mathbf{e}_{\varphi}\right\|_{2, \alpha, \mathcal{N}_{f}^{+}}^{\left(-1-\alpha, \Gamma_{w}^{+}\right)}+\left\|\frac{A}{2 \pi r \sin \theta} \mathbf{e}_{\varphi}\right\|_{1, \alpha, \mathcal{N}_{f}^{+}}^{\left(-\alpha, \Gamma_{w}^{+}\right)}+\|T\|_{1, \alpha, \mathcal{N}_{f}^{+}}^{\left(-\alpha, \Gamma_{w}^{+}\right)}\right)^{2}(3.1
\end{aligned}
$$

and

$$
\begin{aligned}
& \left\|\mathfrak{f}_{1}\left(\Psi \mathbf{e}_{\varphi}, A, T\right)\right\|_{1, \alpha, \Gamma_{e x}}^{\left(-\alpha, \partial \Gamma_{e x}\right)} \\
& \leq C\left(\left\|\Psi \mathbf{e}_{\varphi}\right\|_{2, \alpha, \Gamma_{e x}}^{\left(-1-\alpha, \partial \Gamma_{e x}\right)}+\left\|\frac{A}{2 \pi r \sin \theta} \mathbf{e}_{\varphi}\right\|_{1, \alpha, \Gamma_{e x}}^{\left(-\alpha, \partial \Gamma_{e x}\right)}\right. \\
& \left.\quad+\|T\|_{1, \alpha, \Gamma_{e x}}^{\left(-\alpha, \partial \Gamma_{e x}\right)}\right)^{2}
\end{aligned}
$$

where $C s$ are positive constants depending on $\left(\rho_{0}^{+}, u_{0}^{+}, p_{0}^{+}\right), \gamma, r_{s}, r_{1}, \alpha, \delta_{1}, \delta_{2}$ and $\delta_{4}$. 
Proof. With (3.7), (3.8), (3.17) and the fact that if an axisymmetric vector field $\boldsymbol{a}$ on an axisymmetric connected open set $\Omega$ is in $C^{k}(\Omega)$, then $\nabla \times \boldsymbol{a} \in C^{k-1}(\Omega)$ and $\boldsymbol{a} \cdot \mathbf{e}_{r} \in C^{k}(\Omega)$ (the second one is obtained from Lemma 1), we estimate (3.10) and (3.15) in $C_{\left(1-\alpha, \Gamma_{w}^{+}\right)}^{\alpha}\left(\mathcal{N}_{f}^{+}\right)$, and $C_{\left(-\alpha, \partial \Gamma_{e x}\right)}^{1, \alpha}\left(\Gamma_{e x}\right)$, respectively. Then we obtain the desired result.

Next, we reformulate (B) in terms of $\left(\Psi \mathbf{e}_{\varphi}, A, T\right)$. Using the facts that $L_{0}^{+}=$ $L_{0}^{-}=0$ in $\mathcal{N}_{f}^{+}$and $S_{0}^{+}=\left(g\left(M_{0}^{-2}\right)\right)\left(r_{s}\right) S_{i n}$ in $\mathcal{N}_{f}^{+}$(see (2.14)), we reformulate (B) in terms of $\left(\Psi \mathbf{e}_{\varphi}, A, T\right)$. Then we obtain

$$
\begin{aligned}
& \nabla \times\left(\left(\Phi_{0}^{+}+\Psi\right) \mathbf{e}_{\varphi}\right) \cdot \nabla A=0 \text { in } \mathcal{N}_{f(0)+f_{s}}^{+}, \\
& A=A_{e n, f(0)+f_{s}} \text { on } \Gamma_{f(0)+f_{s}}, \\
& \nabla \times\left(\left(\Phi_{0}^{+}+\Psi\right) \mathbf{e}_{\varphi}\right) \cdot \nabla T=0 \text { in } \mathcal{N}_{f(0)+f_{s}}^{+}, \\
& T=T_{e n, f(0)+f_{s}} \text { on } \Gamma_{f(0)+f_{s}},
\end{aligned}
$$

where

$$
A_{e n, f(0)+f_{s}}:=L_{-} \quad \text { on } \quad \Gamma_{f(0)+f_{s}}
$$

and

$$
T_{e n, f(0)+f_{s}}:=g\left(\left(\frac{\mathbf{u}_{-} \cdot \mathbf{v}_{f(0)+f_{s}}}{c_{-}}\right)^{2}\right) S_{-}-\left(g\left(M_{0}^{-2}\right)\right)\left(r_{s}\right) S_{i n} \quad \text { on } \quad \Gamma_{f(0)+f_{s}} .
$$

For later use, we present the following estimate of $T_{e n, f(0)+f_{s}}$ :

Lemma 6. Let $f(0)$ and $f_{s}^{\prime}$ be a constant and a function in $C_{\left(-\alpha,\left\{\theta=\theta_{1}\right\}\right)}^{1, \alpha}\left(\left(0, \theta_{1}\right)\right)$, respectively. Let $\delta_{5}$ be a positive constant such that if $\left(\rho_{-}, \mathbf{u}_{-}, p_{-}\right)$satisfies (2.19) for $\sigma \in\left(0, \delta_{5}\right]$, then

$$
\sup _{\mathcal{N}}\left|\frac{1}{c_{-}}\right| \leq C
$$

where $C$ is a positive constant depending on $\left(\rho_{0}^{-}, u_{0}^{-}, p_{0}^{-}\right), \gamma, r_{0}, r_{1}$ and $\delta_{5}$. Suppose that $f=f(0)+f_{s}$ satisfies (3.3). Also, suppose that $\left(\rho_{-}, \mathbf{u}_{-}, p_{-}\right)$is an axisymmetric supersonic solution of (2.1) in $\mathcal{N}$ satisfying (2.19) for $\sigma \in\left(0, \delta_{5}\right]$. Then it holds that

$$
\left\|T_{e n, f}\right\|_{1, \alpha, \Gamma_{f}}^{\left(-\alpha, \partial \Gamma_{f}\right)} \leq C\left(\left|f(0)-r_{s}\right|+\left\|f_{s}^{\prime}\right\|_{1, \alpha,\left(0, \theta_{1}\right)}^{\left(-\alpha,\left\{\theta=\theta_{1}\right\}\right)}\right)+C \sigma
$$

where $C$ s are positive constants depending on $\left(\rho_{0}^{-}, u_{0}^{-}, p_{0}^{-}\right), \gamma, r_{0}, r_{s}, r_{1}, \theta_{1}, \alpha, \delta_{1}$ and $\delta_{5}$. 
Proof. In this proof, $C$ s denote positive constants depending on the whole or a part of $\left(\rho_{0}^{-}, u_{0}^{-}, p_{0}^{-}\right), \gamma, r_{0}, r_{s}, r_{1}, \theta_{1}, \alpha, \delta_{1}$ and $\delta_{5}$. Each $C$ in different situations differs from each other.

By the fact that $\left(\rho_{-}, \mathbf{u}_{-}, p_{-}\right)$and $f=f(0)+f_{s}$ are axisymmetric, $T_{e n, f}$ defined in (3.21) can be regarded as a function of $\theta$. As a function of $\theta, T_{e n, f}$ can be written as

$$
\begin{aligned}
T_{e n, f}= & \int_{0}^{1}\left(g\left(M_{0}^{-2}\right)\right)^{\prime}\left(t f(\theta)+(1-t) r_{s}\right) S_{\text {in }} \mathrm{d} t\left(f(\theta)-r_{s}\right) \\
& +\left(g\left(\left(\frac{\mathbf{u}_{-} \cdot \mathbf{v}_{f}(\theta)}{c_{-}}\right)^{2}\right) S_{-}\right)(f(\theta), \theta)-\left(g\left(M_{0}^{-2}\right)\right)(f(\theta), \theta) S_{i n} \\
= & :(a)+(b) .
\end{aligned}
$$

To estimate $\left\|T_{e n, f}\right\|_{1, \alpha, \Gamma_{f}}^{\left(-\alpha, \partial \Gamma_{f}\right)}$, we estimate $(a)$ and $(b)$ in $C_{\left(-\alpha,\left\{\theta=\theta_{1}\right\}\right)}^{1, \alpha}\left(\left(0, \theta_{1}\right)\right)$, respectively.

Since an estimate of $(a)$ in $C_{\left(-\alpha,\left\{\theta=\theta_{1}\right\}\right)}^{1, \alpha}\left(\left(0, \theta_{1}\right)\right)$ is obtained directly:

$$
\|(a)\|_{1, \alpha,\left(0, \theta_{1}\right)}^{\left(-\alpha,\left\{\theta=\theta_{1}\right\}\right)} \leq C\left(\left|f(0)-r_{s}\right|+\left\|f_{S}^{\prime}\right\|_{1, \alpha,\left(0, \theta_{1}\right)}^{\left(-\alpha,\left\{\theta=\theta_{1}\right\}\right)}\right),
$$

where we used $\left\|f_{S}\right\|_{1, \alpha,\left(0, \theta_{1}\right)}^{\left(-\alpha,\left\{\theta=\theta_{1}\right\}\right)} \leq C\left\|f_{S}^{\prime}\right\|_{1, \alpha,\left(0, \theta_{1}\right)}^{\left(-\alpha,\left\{\theta=\theta_{1}\right\}\right)}$, we only estimate $(b)$ in $C_{\left(-\alpha,\left\{\theta=\theta_{1}\right\}\right)}^{1, \alpha}\left(\left(0, \theta_{1}\right)\right)$.

Estimate of $(b)$ in $C_{\left(-\alpha,\left\{\theta=\theta_{1}\right\}\right)}^{1, \alpha}\left(\left(0, \theta_{1}\right)\right)$ :

Decompose $(b)$ into two parts:

$$
\left(g\left(\left(\frac{\mathbf{u}_{-} \cdot \mathbf{e}_{r}}{c_{-}}\right)^{2}\right) S_{-}\right)(f(\theta), \theta)-\left(g\left(M_{0}^{-2}\right)\right)(f(\theta), \theta) S_{i n}=:(b)_{1}
$$

and

$$
\left(g\left(\left(\frac{\mathbf{u}_{-} \cdot \mathbf{v}_{f}}{c_{-}}\right)^{2}\right) S_{-}\right)(f(\theta), \theta)-\left(g\left(\left(\frac{\mathbf{u}_{-} \cdot \mathbf{e}_{r}}{c_{-}}\right)^{2}\right) S_{-}\right)(f(\theta), \theta)=:(b)_{2} .
$$

With (2.19) for $\sigma \in\left(0, \delta_{5}\right]$ and (3.3), we estimate $(b)_{1}$ in $C_{\left(-\alpha,\left\{\theta=\theta_{1}\right\}\right)}^{1, \alpha}\left(\left(0, \theta_{1}\right)\right)$. Then we obtain

$$
\left\|(b)_{1}\right\|_{1, \alpha,\left(0, \theta_{1}\right)}^{\left(-\alpha,\left\{\theta=\theta_{1}\right\}\right)} \leq C \sigma .
$$

Write $(b)_{2}$ as

$$
\begin{aligned}
\int_{0}^{1} & g^{\prime}\left(\left(\frac{\mathbf{u}_{-}}{c_{-}} \cdot\left(t \boldsymbol{v}_{f}+(1-t) \mathbf{e}_{r}\right)\right)^{2}\right) 2\left(\frac{\mathbf{u}_{-}}{c_{-}} \cdot\left(t \mathbf{v}_{f}+(1-t) \mathbf{e}_{r}\right)\right) S_{-} \mathrm{d} t \\
& \frac{\mathbf{u}_{-}}{c_{-}} \cdot\left(\boldsymbol{v}_{f}-\mathbf{e}_{r}\right) .
\end{aligned}
$$


By $\boldsymbol{v}_{f}=\frac{\mathbf{e}_{r}-\frac{f^{\prime}}{f} \mathbf{e}_{\theta}}{\sqrt{1+\left(\frac{f^{\prime}}{f}\right)^{2}}}, \boldsymbol{v}_{f}-\mathbf{e}_{r}$ can be written as

$$
\boldsymbol{v}_{f}-\mathbf{e}_{r}=\left(\int_{0}^{1}-\frac{1}{2}\left(1+t\left(\frac{f^{\prime}}{f}\right)^{2}\right)^{-\frac{3}{2}} \mathrm{~d} t\left(\frac{f^{\prime}}{f}\right)^{2}\right) \mathbf{e}_{r}-\frac{\frac{f^{\prime}}{f}}{\sqrt{1+\left(\frac{f^{\prime}}{f}\right)^{2}}} \mathbf{e}_{\theta} .
$$

Substitute this expression of $\boldsymbol{v}_{f}-\mathbf{e}_{r}$ into $\boldsymbol{v}_{f}-\mathbf{e}_{r}$ in (3.25) and then estimate (3.25) in $C_{\left(-\alpha,\left\{\theta=\theta_{1}\right\}\right)}^{1, \alpha}\left(\left(0, \theta_{1}\right)\right)$ with (2.19) for $\sigma \in\left(0, \delta_{5}\right]$ and (3.3). Then we obtain

$$
\left\|(b)_{2}\right\|_{1, \alpha,\left(0, \theta_{1}\right)}^{\left(-\alpha,\left\{\theta=\theta_{1}\right\}\right)} \leq C\left(\left\|f_{s}^{\prime}\right\|_{1, \alpha,\left(0, \theta_{1}\right)}^{\left(-\alpha,\left\{\theta=\theta_{1}\right\}\right)}\right)^{2}+C \delta_{5}\left\|f_{S}^{\prime}\right\|_{1, \alpha,\left(0, \theta_{1}\right)}^{\left(-\alpha,\left\{\theta=\theta_{1}\right\}\right)} .
$$

Using the fact that $\left\|f_{s}^{\prime}\right\|_{1, \alpha,\left(0, \theta_{1}\right)}^{\left(-\alpha,\left(0, \theta_{1}\right)\right)} \leq C\left\|f-r_{s}\right\|_{2, \alpha, \Lambda}^{(-1-\alpha, \partial \Lambda)} \leq C \delta_{1}$, we get from this estimate

$$
\left\|(b)_{2}\right\|_{1, \alpha,\left(0, \theta_{1}\right)}^{\left(-\alpha,\left\{\theta=\theta_{1}\right\}\right)} \leq C\left\|f_{S}^{\prime}\right\|_{1, \alpha,\left(0, \theta_{1}\right)}^{\left(-\alpha,\left\{\theta=\theta_{1}\right\}\right)} .
$$

Combining (3.24) and (3.26), we obtain

$$
\|(b)\|_{1, \alpha,\left(0, \theta_{1}\right)}^{\left(-\alpha,\left\{\theta=\theta_{1}\right\}\right)} \leq C\left\|f_{S}^{\prime}\right\|_{1, \alpha,\left(0, \theta_{1}\right)}^{\left(-\alpha,\left(0, \theta_{1}\right)\right)}+C \sigma .
$$

From the facts that $\partial_{\theta} T_{e n, f}(0)=0$ and $(a),(b) \in C_{\left(-\alpha,\left\{\theta=\theta_{1}\right\}\right)}^{1, \alpha}\left(\left(0, \theta_{1}\right)\right)$ (obtained from (3.23) and (3.27)), we see that $T_{e n, f} \in C_{\left(-\alpha, \partial \Gamma_{f}\right)}^{1, \alpha}\left(\Gamma_{f}\right)$ (see Lemma 1). So, $\left\|T_{e n, f}\right\|_{1, \alpha, \Gamma_{f}}^{\left(-\alpha, \partial \Gamma_{f}\right)}$ can be estimated by estimating $\left\|T_{e n, f}\right\|_{1, \alpha,\left(0, \theta_{1}\right)}^{\left(-\alpha,\left\{\theta=\theta_{1}\right\}\right)}$. Using this fact, we obtain from (3.23) and (3.27) (3.22). This finishes the proof.

From $\left(\mathrm{A}^{\prime}\right),\left(\mathrm{B}^{\prime}\right)$, the Pseudo Free Boundary Problem is naturally derived. We explain this below.

For a given $\left(\rho_{-}, \mathbf{u}_{-}, p_{-}, p_{e x}\right)$, find $\left(\Psi \mathbf{e}_{\varphi}, A, T\right)$ satisfying $\left(\mathrm{A}^{\prime}\right),\left(\mathrm{B}^{\prime}\right)$ using an iteration scheme for a fixed boundary problem (for example, in a fixed domain $\mathcal{N}_{f(0)+f_{s}}^{+}$, for a given $\Psi \mathbf{e}_{\varphi}$, solve $\left(\mathrm{B}^{\prime}\right)$, substitute the resultant $A$ and $T$ and the previously given $\Psi \mathbf{e}_{\varphi}$ into the right hand sides of ( $\left.\mathrm{A}^{\prime}\right)$, obtain a new $\Psi \mathbf{e}_{\varphi}$ by solving the resultant $\left(\mathrm{A}^{\prime}\right)$ and show that a new $\Psi \mathbf{e}_{\varphi}$ is equal to the given $\Psi \mathbf{e}_{\varphi}$ using a fixed point argument). Then $\left(\Psi \mathbf{e}_{\varphi}, A, T\right)$ we find in this way does not satisfy the fifth equation of $\left(\mathrm{A}^{\prime}\right)$ in general. Thus, this kind of iteration scheme does not give a subsonic solution of (2.43)-(2.45) satisfying (2.54) in general. To find a subsonic solution satisfying (2.54), an iteration scheme that gives a proper $\left(\Psi \mathbf{e}_{\varphi}, A, T\right)$ on $\Gamma_{e x}$ is necessary. From the fifth equation of $\left(\mathrm{A}^{\prime}\right)$ and the facts that the entropy at a point on a shock location in the subsonic side is conserved along the streamline passing through that point and the entropy of the downstream subsonic solution of a radial transonic shock solution in a divergent nozzle on a shock location monotonically increases as a shock location moves toward the exit (see Lemma 2), it is seen that we can find $\left(\Psi \mathbf{e}_{\varphi}, A, T\right)$ satisfying the fifth equation of $\left(\mathrm{A}^{\prime}\right)$ by adjusting $f(0)$ to vary $S$ on $\Gamma_{e x}$. From this fact, Problem 3 is derived.

$\left(\mathrm{A}^{\prime}\right)$ and $\left(\mathrm{B}^{\prime}\right)$ are of the form of one linear boundary value problem for a singular elliptic equation (this will be seen in the next subsection) and two initial 
value problems of a transport equation whose coefficient is an axisymmetric and divergence-free vector field, respectively. We will study these problems, seperately, in $\S 3.2$ and $\S 3.3$.

\subsection{Linear boundary value problem for a singular elliptic equation}

Fix the right hand sides of the first and fourth equation in $\left(\mathrm{A}^{\prime}\right)$ with the fifth equation in $\left(\mathrm{A}^{\prime}\right)$ satisfied. Then we obtain

$$
\begin{aligned}
& \nabla \times\left(\frac{1}{\rho_{0}^{+}}\left(1+\frac{u_{0}^{+} \mathbf{e}_{r} \otimes u_{0}^{+} \mathbf{e}_{r}}{c_{0}^{+2}-u_{0}^{+2}}\right) \nabla \times\left(\Psi \mathbf{e}_{\varphi}\right)\right)=\boldsymbol{F} \text { in } \mathcal{N}_{f}^{+}, \\
& \Psi \mathbf{e}_{\varphi}=\left\{\begin{array}{l}
h_{1} \mathbf{e}_{\varphi} \text { on } \Gamma_{f}, \\
\frac{f\left(\theta_{1}\right) h_{1}\left(f\left(\theta_{1}\right), \theta_{1}\right)}{r} \mathbf{e}_{\varphi} \text { on } \Gamma_{w}^{+}, \\
h_{2} \mathbf{e}_{\varphi} \text { on } \Gamma_{e x}
\end{array}\right.
\end{aligned}
$$

where $f, \boldsymbol{F}$ and $h_{i} \mathbf{e}_{\varphi}$ for $i=1,2$ are functions given in Lemma 7. Since (3.28) is expressed as

$$
\begin{aligned}
& \left(-\frac{1}{\rho_{0}^{+}}\left(\Delta \Psi-\frac{\Psi}{r^{2} \sin ^{2} \theta}\right)+\frac{\partial_{r} \rho_{0}^{+}}{\rho_{0}^{+2} r} \partial_{r}(r \Psi)\right. \\
& \left.-\frac{u_{0}^{+2}}{\rho_{0}^{+}\left(c_{0}^{+2}-u_{0}^{+2}\right) r^{2}}\left(\frac{1}{\sin \theta} \partial_{\theta}\left(\sin \theta \partial_{\theta} \Psi\right)-\frac{\Psi}{\sin ^{2} \theta}\right)\right) \mathbf{e}_{\varphi}=\boldsymbol{F},
\end{aligned}
$$

(3.28), (3.29) is a linear boundary value problem for a singular equation as a problem for $\Psi$. Thus, the standard elliptic theorems cannot be applied to this problem as a problem for $\Psi$. We resolve this problem by dealing with (3.28), (3.29) as a boundary value problem for an elliptic system.

The following is the main result in this subsection.

Lemma 7. Let $\alpha \in\left(\frac{2}{3}, 1\right)$. Suppose that $f$ is as in Lemma 4 and satisfy $f^{\prime}\left(\theta_{1}\right)=0$. Also, suppose that $\boldsymbol{F}: \mathcal{N}_{f}^{+} \rightarrow \mathbb{R}^{3}$ is a function in $C_{\left(1-\alpha, \Gamma_{w}^{+}\right)}^{\alpha}\left(\mathcal{N}_{f}^{+}\right)$having the form

$$
\boldsymbol{F}=\sum_{i} A^{i} \partial_{r} B^{i} \mathbf{e}_{\varphi}+\sum_{i} C^{i} \partial_{\theta} D^{i} \mathbf{e}_{\varphi}+E \frac{\partial_{\theta}(F \sin \theta)}{\sin \theta} \mathbf{e}_{\varphi}
$$

where $A^{i}, B^{i}, C^{i}, D^{i}, E$ and $F$ are axisymmetric functions satisfying

$$
\begin{aligned}
& A^{i} \in C_{\left(-\alpha, \Gamma_{w}^{+}\right)}^{1, \alpha}\left(\mathcal{N}_{f}^{+}\right), B^{i} \mathbf{e}_{\theta} \in C_{\left(-\alpha, \Gamma_{w}^{+}\right)}^{1, \alpha}\left(\mathcal{N}_{f}^{+}\right), C^{i} \in C_{\left(-\alpha, \Gamma_{w}^{+}\right)}^{1, \alpha}\left(\mathcal{N}_{f}^{+}\right), \\
& D^{i} \in C_{\left(-\alpha, \Gamma_{w}^{+}\right)}^{1, \alpha}\left(\mathcal{N}_{f}^{+}\right), \quad E \mathbf{e}_{\varphi} \in C^{1, \alpha}\left(\overline{\mathcal{N}_{f}^{+}}\right) \text {and } F \mathbf{e}_{\varphi} \in C^{1, \alpha}\left(\overline{\mathcal{N}_{f}^{+}}\right) .
\end{aligned}
$$

Finally, suppose that $h_{1} \mathbf{e}_{\varphi}: \Gamma_{f} \rightarrow \mathbb{R}^{3}$ and $h_{2} \mathbf{e}_{\varphi}: \Gamma_{\text {ex }} \rightarrow \mathbb{R}^{3}$ are axisymmetric functions in $C_{\left(-1-\alpha, \partial \Gamma_{f}\right)}^{2, \alpha}\left(\Gamma_{f}\right)$ and $C_{\left(-1-\alpha, \partial \Gamma_{e x}\right)}^{2, \alpha}\left(\Gamma_{e x}\right)$, respectively, and satisfy $\frac{f\left(\theta_{1}\right) h_{1}\left(f\left(\theta_{1}\right), \theta_{1}\right)}{r_{1}}=h_{2}\left(r_{1}, \theta_{1}\right)$. Then the boundary value problem (3.28), (3.29) has 
a unique axisymmetric $C_{\left(-1-\alpha, \Gamma_{w}^{+}\right)}^{2, \alpha}\left(\mathcal{N}_{f}^{+}\right)$solution $\Psi \mathbf{e}_{\varphi}$. Furthermore, the solution $\Psi \mathbf{e}_{\varphi}$ satisfies

$$
\begin{aligned}
& \left\|\Psi \mathbf{e}_{\varphi}\right\|_{2, \alpha, \mathcal{N}_{f}^{+}}^{\left(-1-\alpha, \Gamma_{w}^{+}\right)} \\
& \leq C\left(\|\boldsymbol{F}\|_{\alpha, \mathcal{N}_{f}^{+}}^{\left(1-\alpha, \Gamma_{w}^{+}\right)}+\sum_{i=1,2,3} F_{i}+\left\|h_{1} \mathbf{e}_{\varphi}\right\|_{2, \alpha, \Gamma_{f}}^{\left(-1-\alpha, \partial \Gamma_{f}\right)}\right. \\
& \left.\quad+\left\|h_{2} \mathbf{e}_{\varphi}\right\|_{2, \alpha, \Gamma_{e x}}^{\left(-1-\alpha, \partial \Gamma_{e x}\right)}\right)
\end{aligned}
$$

where $C$ is a positive constant depending only on $\left(\rho_{0}^{+}, u_{0}^{+}, p_{0}^{+}\right), \gamma, r_{s}, r_{1}, \theta_{1}$ and $\alpha$, and

$$
\begin{aligned}
& F_{1}=\sum_{i}\left\|A^{i}\right\|_{W^{1,3}\left(\mathcal{N}_{f}^{+}\right) \cap L^{\infty}\left(\mathcal{N}_{f}^{+}\right)}\left\|B^{i} \mathbf{e}_{\theta}\right\|_{\alpha, \mathcal{N}_{f}^{+}} \\
& F_{2}=\sum_{i}\left\|C^{i}\right\|_{W^{1,3}\left(\mathcal{N}_{f}^{+}\right) \cap L^{\infty}\left(\mathcal{N}_{f}^{+}\right)}\left\|D^{i}\right\|_{\alpha, \mathcal{N}_{f}^{+}}, \\
& F_{3}=\left\|E \frac{\partial_{\theta}(F \sin \theta)}{\sin \theta} \mathbf{e}_{\varphi}\right\|_{L^{q}\left(\mathcal{N}_{f}^{+}\right)}
\end{aligned}
$$

for $q=\frac{3}{1-\alpha}$ with $\|\cdot\|_{W^{1,3}\left(\mathcal{N}_{f}^{+}\right) \cap L^{\infty}\left(\mathcal{N}_{f}^{+}\right)}:=\|\cdot\|_{W^{1,3}\left(\mathcal{N}_{f}^{+}\right)}+\|\cdot\|_{L^{\infty}\left(\mathcal{N}_{f}^{+}\right)} \cdot$

Remark 7. The form of $\boldsymbol{F}$ given in (3.31) is obtained from $\boldsymbol{F}_{2}\left(\Psi \mathbf{e}_{\varphi}, A, T\right)$ in (3.9). This form will be used in the proof of Lemma 11.

To avoid the singularity issue in (3.28), (3.29), we deal with (3.28), (3.29) as a boundary value problem for a vector equation. From $\nabla \times\left(\nabla \times\left(\Psi \mathbf{e}_{\varphi}\right)\right)=-\Delta\left(\Psi \mathbf{e}_{\varphi}\right)$, we expected that (3.28) can be transformed into a form of an elliptic system. We, motivated by the work in [4], thought that if (3.28) can be transformed into a solvable elliptic system form, then the unique existence and regularity of solutions of (3.28), (3.29) can be obtained by obtaining those of solutions of the elliptic system form of (3.28), (3.29) as a boundary value problem for an elliptic system.

For this argument to hold, it is needed to find a solvable elliptic system form of (3.28). For computational convenience to find such a form and for our later argument (reflection argument in the proof of Lemma 10 and Lemma 11), we use the following tensor notation:

\section{Tensor notation}

Let $\boldsymbol{a} \otimes \boldsymbol{b}=\boldsymbol{a} \boldsymbol{b}^{T}$ for $\boldsymbol{a}, \boldsymbol{b} \in \mathbb{R}^{3}$. Then $\boldsymbol{a} \otimes \boldsymbol{b}$ is a linear map from $\mathbb{R}^{3}$ to $\mathbb{R}^{3}$ and any linear map from $\mathbb{R}^{3}$ to $\mathbb{R}^{3}$ can be represented using this operator. This notation can be extended to represent any linear map from $\mathbb{R}^{3 \times 3}$ to $\mathbb{R}^{3 \times 3}$. For any $\boldsymbol{a}, \boldsymbol{b}, \boldsymbol{c}$, $\boldsymbol{d} \in \mathbb{R}^{3}$, let $\boldsymbol{a} \otimes \boldsymbol{b} \otimes \boldsymbol{c} \otimes \boldsymbol{d}$ be an operator satisfying

$$
(\boldsymbol{a} \otimes \boldsymbol{b} \otimes \boldsymbol{c} \otimes \boldsymbol{d})(\boldsymbol{e} \otimes \boldsymbol{f})=(\boldsymbol{d} \cdot \boldsymbol{e})(\boldsymbol{c} \cdot \boldsymbol{f}) \boldsymbol{a} \otimes \boldsymbol{b},
$$

where $\boldsymbol{e}, \boldsymbol{f} \in \mathbb{R}^{3}$. Then $\boldsymbol{a} \otimes \boldsymbol{b} \otimes \boldsymbol{c} \otimes \boldsymbol{d}$ is a linear map from $\mathbb{R}^{3 \times 3}$ to $\mathbb{R}^{3 \times 3}$ and any linear map from $\mathbb{R}^{3 \times 3}$ to $\mathbb{R}^{3 \times 3}$ can be represented using this operator. 
By direct computation done by using the above tensor notation, we found an elliptic system form of (3.28). We found the following form of (3.28)

$$
\begin{aligned}
& \operatorname{div}\left(\frac{c_{0}^{+2}}{\rho_{0}^{+}\left(c_{0}^{+2}-u_{0}^{+2}\right)}\left(\boldsymbol{I}-\frac{u_{0}^{+2}}{c_{0}^{+2}}\left(\mathcal{I} \otimes \mathbf{e}_{r} \otimes \mathbf{e}_{r} \otimes \mathcal{I}\right)\right) D\left(\Psi \mathbf{e}_{\varphi}\right)\right) \\
& -\frac{\partial_{r} \rho_{0}^{+}}{\rho_{0}^{+2} r} \Psi \mathbf{e}_{\varphi}=-\boldsymbol{F} \text { in } \mathcal{N}_{f}^{+},
\end{aligned}
$$

where $\boldsymbol{I}$ is the identity map from $\mathbb{R}^{3 \times 3}$ to $\mathbb{R}^{3 \times 3}$ and $\mathcal{I} \otimes \mathbf{e}_{r} \otimes \mathbf{e}_{r} \otimes \mathcal{I}$ is a linear map from $\mathbb{R}^{3 \times 3}$ to $\mathbb{R}^{3 \times 3}$ satisfing

$$
\left(\mathcal{I} \otimes \mathbf{e}_{r} \otimes \mathbf{e}_{r} \otimes \mathcal{I}\right)(\boldsymbol{a} \otimes \boldsymbol{b})=\left(\boldsymbol{b} \cdot \mathbf{e}_{r}\right) \boldsymbol{a} \otimes \mathbf{e}_{r}
$$

for any $\boldsymbol{a}, \boldsymbol{b} \in R^{3}$ (see the definition of $\mathcal{I} \otimes \boldsymbol{a} \otimes \boldsymbol{b} \otimes \mathcal{I}$ for any $\boldsymbol{a}, \boldsymbol{b} \in \mathbb{R}^{3}$ in (5.4)). By $M_{0}^{+}<1$ in $\mathcal{N}_{f}^{+}$and the boundedness of $\left(\rho_{0}^{+}, u_{0}^{+}, p_{0}^{+}\right)$in $\mathcal{N}_{f}^{+}$for $\mathcal{N}_{f}^{+} \subset \mathcal{N}_{r_{s}-\delta_{1}}^{+}$, there exist positive constants $\mu$ and $\mathcal{M}$ such that

$$
\begin{aligned}
\mu|\boldsymbol{\xi}|^{2} & \leq \sum_{\alpha, \beta, i, j=1}^{3}\left[\frac{c_{0}^{+^{2}}}{\rho_{0}^{+}\left(c_{0}^{+^{2}}-u_{0}^{+^{2}}\right)}\left(\boldsymbol{I}-\frac{u_{0}^{+2}}{c_{0}^{+^{2}}}\left(\mathcal{I} \otimes \mathbf{e}_{r} \otimes \mathbf{e}_{r} \otimes \mathcal{I}\right)\right)\right]_{i j}^{\alpha \beta} \xi_{\alpha}^{i} \xi_{\beta}^{j} \\
& \leq \mathcal{M}|\boldsymbol{\xi}|^{2} \text { in } \mathcal{N}_{f}^{+}
\end{aligned}
$$

for any $\boldsymbol{\xi} \in \mathbb{R}^{3 \times 3}$ where $|\boldsymbol{\xi}|=\sqrt{\sum_{i, j=1}^{3}\left|\xi_{j}^{i}\right|^{2}}$ with $\boldsymbol{\xi}=\left[\xi_{j}^{i}\right]$. And by $\partial_{r} \rho_{0}^{+}>0$ in $\mathcal{N}_{f}^{+}$for $\mathcal{N}_{f}^{+} \subset \mathcal{N}_{r_{s}-\delta_{1}}^{+}$(see Remark 2),

$$
\frac{\partial_{r} \rho_{0}^{+}}{\rho_{0}^{+2} r}>0 \text { in } \mathcal{N}_{f}^{+} \text {. }
$$

Hence, (3.35) is a form of a solvable elliptic system for a Dirichlet boundary condition.

We obtain the unique existence and regularity of solutions of (3.28), (3.29) by obtaining those of solutions of (3.35), (3.29) as a boundary value problem for an elliptic system. The result of the unique existence and regularity of solutions of (3.35), (3.29) as a boundary value problem for an elliptic system is given in the following lemma:

Lemma 8. Under the assumptions as in Proposition 7, the boundary value problem

$$
\begin{aligned}
& \operatorname{div}\left(\frac{c_{0}^{+2}}{\rho_{0}^{+}\left(c_{0}^{+2}-u_{0}^{+2}\right)}\left(\boldsymbol{I}-\frac{u_{0}^{+2}}{c_{0}^{+2}}\left(\mathcal{I} \otimes \mathbf{e}_{r} \otimes \mathbf{e}_{r} \otimes \mathcal{I}\right)\right) D \boldsymbol{U}\right)-\frac{\partial_{r} \rho_{0}^{+}}{\rho_{0}^{+2} r} \boldsymbol{U}=-\boldsymbol{F} \\
& \text { in } \mathcal{N}_{f}^{+}, \\
& \boldsymbol{U}=\left\{\begin{array}{lll}
h_{1} \mathbf{e}_{\varphi} \text { on } \Gamma_{f}, \\
\frac{f\left(\theta_{1}\right) h_{1}\left(f\left(\theta_{1}\right), \theta_{1}\right)}{r} \mathbf{e}_{\varphi} \text { on } \Gamma_{w}^{+}, \\
h_{2} \mathbf{e}_{\varphi} \text { on } \Gamma_{\text {ex }},
\end{array}\right.
\end{aligned}
$$


has a unique $C_{\left(-1-\alpha, \Gamma_{w}^{+}\right)}^{2, \alpha}\left(\mathcal{N}_{f}^{+}\right)$solution $\boldsymbol{U}$, and this solution $\boldsymbol{U}$ satisfies

$$
\begin{aligned}
& \|\boldsymbol{U}\|_{2, \alpha, \mathcal{N}_{f}^{+}}^{\left(-1-\alpha, \Gamma_{w}^{+}\right)} \\
& \leq \underbrace{C\left(\|\boldsymbol{F}\|_{\alpha, \mathcal{N}_{f}^{+}}^{\left(1-\alpha, \Gamma_{w}^{+}\right)}+\sum_{i=1,2,3} F_{i}+\left\|h_{1} \mathbf{e}_{\varphi}\right\|_{2, \alpha, \Gamma_{f}}^{\left(-1-\alpha, \partial \Gamma_{f}\right)}+\left\|h_{2} \mathbf{e}_{\varphi}\right\|_{2, \alpha, \Gamma_{e x}}^{\left(-1-\alpha, \partial \Gamma_{e x}\right)}\right)}_{=: C^{*}},
\end{aligned}
$$

where $C$ is a positive constant depending on $\left(\rho_{0}^{+}, u_{0}^{+}, p_{0}^{+}\right), \gamma, r_{s}, r_{1}, \theta_{1}$ and $\alpha$ and $F_{i}$ for $i=1,2,3$ are constants given in Lemma 11 . This solution $\boldsymbol{U}$ is of the form $\Psi(r, \theta) \mathbf{e}_{\varphi}$.

One can see that that Lemma 7 is obtained from Lemma 8. To prove Lemma 7, in the remainder of this subsection, we prove Lemma 8.

To prove Lemma 8, we transform (3.37), (3.38) into the $\mathbf{0}$ boundary problem

$$
\begin{aligned}
& \operatorname{div}\left(\boldsymbol{A} D \boldsymbol{U}^{\sharp}\right)-d \boldsymbol{U}^{\sharp}=-\boldsymbol{F}-\operatorname{div}(\boldsymbol{A D h})+d \boldsymbol{h}\left(=: \boldsymbol{F}^{\sharp}\right) \text { in } \mathcal{N}_{f}^{+}, \\
& \boldsymbol{U}^{\sharp}=0 \text { on } \partial \mathcal{N}_{f}^{+},
\end{aligned}
$$

where $\boldsymbol{U}^{\sharp}:=\boldsymbol{U}-\boldsymbol{h}, \boldsymbol{A}=\frac{c_{0}^{+2}}{\rho_{0}^{+}\left(c_{0}^{+2}-u_{0}^{+2}\right)}\left(\boldsymbol{I}-\frac{u_{0}^{+2}}{c_{0}^{+2}}\left(\mathcal{I} \otimes \mathbf{e}_{r} \otimes \mathbf{e}_{r} \otimes \mathcal{I}\right)\right), d:=\frac{\partial_{r} \rho_{0}^{+}}{\rho_{0}^{+2} r}$ and $\boldsymbol{h}:=\frac{(r-f(\theta)) \frac{r_{1}}{r} h_{2} \mathbf{e}_{\varphi}+\left(r_{1}-r\right) \frac{f(\theta)}{r} h_{1} \mathbf{e}_{\varphi}}{r_{1}-f(\theta)}$. One can see that Lemma 8 can be proved by showing (3.40), (3.41) has a unique weak solution, the weak solution of (3.40), (3.41) is in $C_{\left(-1-\alpha, \Gamma_{w}^{+}\right)}^{2, \alpha}\left(\mathcal{N}_{f}^{+}\right)$and the $C_{\left(-1-\alpha, \Gamma_{w}^{+}\right)}^{2, \alpha}\left(\mathcal{N}_{f}^{+}\right)$solution of (3.40), (3.41) is of the form $\Psi(r, \theta) \mathbf{e}_{\varphi}$. Hereafter, we prove these statements.

We first prove the unique existence of weak solution of (3.40), (3.41).

Lemma 9. Under the assumptions as in Proposition 7, the boundary value problem (3.40), (3.41) has a unique weak solution $\boldsymbol{U}^{\sharp} \in H_{0}^{1}\left(\mathcal{N}_{f}^{+}\right)$. Furthermore, $\boldsymbol{U}^{\sharp}$ satisfies

$$
\left\|\boldsymbol{U}^{\sharp}\right\|_{H^{1}\left(\mathcal{N}_{f}^{+}\right)} \leq C\left\|\boldsymbol{F}^{\sharp}\right\|_{L^{2}\left(\mathcal{N}_{f}^{+}\right)},
$$

where $C$ is a positive constant depending on $\left(\rho_{0}^{+}, u_{0}^{+}, p_{0}^{+}\right), \gamma$ and $\mathcal{N}_{f}^{+}$.

Proof. Write (3.40), (3.41) in the form

$$
\left(B\left[\boldsymbol{U}^{\sharp}, \boldsymbol{\xi}\right]:=\right) \int_{\mathcal{N}_{f}^{+}} \boldsymbol{A} D \boldsymbol{U}^{\sharp} D \boldsymbol{\xi}+d \boldsymbol{U}^{\sharp} \boldsymbol{\xi}=\int_{\mathcal{N}_{f}^{+}} \boldsymbol{F}^{\sharp} \boldsymbol{\xi}\left(=:<\boldsymbol{F}^{\sharp}, \boldsymbol{\xi}>\right)
$$

for all $\boldsymbol{\xi} \in H_{0}^{1}\left(\mathcal{N}_{f}^{+}\right)$. Then $B$ is a bilinear map satisfying $B\left[\boldsymbol{U}^{\sharp}, \boldsymbol{\xi}\right]$ $\leq C\left\|\boldsymbol{U}^{\sharp}\right\|_{H^{1}\left(\mathcal{N}_{f}^{+}\right)}\|\boldsymbol{\xi}\|_{H^{1}\left(\mathcal{N}_{f}^{+}\right)}$for a constant $C>0$ and

$$
\underline{\mu}\left\|\boldsymbol{U}^{\sharp}\right\|_{H^{1}\left(\mathcal{N}_{f}^{+}\right)}^{2} \leq B\left[\boldsymbol{U}^{\sharp}, \boldsymbol{U}^{\sharp}\right],
$$


where $\underline{\mu}=\min \left(\mu, \min _{\mathcal{N}_{f}^{+}} d\right)>0$ with $\mu$ given in (3.36). And since $h_{1} \mathbf{e}_{\varphi} \in$ $C_{\left(-1-\alpha, \partial \Gamma_{f}\right)}^{2, \alpha}\left(\Gamma_{f}\right), h_{2} \mathbf{e}_{\varphi} \in C_{\left(-1-\alpha, \partial \Gamma_{e x}\right)}^{2, \alpha}\left(\Gamma_{e x}\right)$ and $\boldsymbol{F} \in C_{\left(1-\alpha, \Gamma_{w}^{+}\right)}^{\alpha}\left(\mathcal{N}_{f}^{+}\right)$for $\alpha \in$ $\left(\frac{2}{3}, 1\right), \boldsymbol{F}^{\sharp} \in L^{2}\left(\mathcal{N}_{f}^{+}\right)$and thus $<\boldsymbol{F}^{\sharp}, \boldsymbol{\xi}>$ is a bounded linear functional on $H_{0}^{1}\left(\mathcal{N}_{f}^{+}\right)$. With these facts, we apply the Lax-Milgram Theorem to (3.42). Then we obtain that there exists a unique $\boldsymbol{U}^{\sharp} \in H_{0}^{1}\left(\mathcal{N}_{f}^{+}\right)$such that (3.42) holds for all $\xi \in H_{0}^{1}\left(\mathcal{N}_{f}^{+}\right)$. This finishes the proof.

We next prove that this weak solution is in $C_{\left(-1-\alpha, \Gamma_{w}^{+}\right)}^{2, \alpha}\left(\mathcal{N}_{f}^{+}\right)$. For this, we prove that the weak solution of (3.40), (3.41) is in $C^{\beta}\left(\overline{\mathcal{N}_{f}^{+}}\right)$for any $\beta \in(0,1)$ and $C^{1, \alpha}\left(\overline{\mathcal{N}_{f}^{+}}\right)$.

Lemma 10. Under the assumptions as in Proposition 7, let $\boldsymbol{U}^{\sharp}$ be the weak solution of the boundary value problem (3.40), (3.41). Then for any $\beta \in(0,1)$,

$$
\left\|\boldsymbol{U}^{\sharp}\right\|_{\beta, \mathcal{N}_{f}^{+}} \leq C\left(\left\|\boldsymbol{F}^{\sharp}\right\|_{L^{p}\left(\mathcal{N}_{f}^{+}\right)}+\left\|\boldsymbol{U}^{\sharp}\right\|_{H^{1}\left(\mathcal{N}_{f}^{+}\right)}\right)
$$

for $p=\frac{3}{2-\beta}$ where $C$ is a positive constant depending on $\mu, \mathcal{M}, \tau,\|d\|_{L^{3}\left(\mathcal{N}_{f}^{+}\right)}$ and $\mathcal{N}_{f}^{+}$, and $\tau$ is the modulus of continuity of $\boldsymbol{A}$ in $\mathcal{N}_{f}^{+}$given as

$$
\tau(t)=\sup _{\substack{\mathrm{x}, \mathrm{y} \in \mathcal{N}_{f}^{+},|\mathrm{x}-\mathrm{y}| \leq t}}\left(\sum\left|A_{i j}^{\alpha \beta}(\mathrm{x})-A_{i j}^{\alpha \beta}(\mathrm{y})\right|^{2}\right)^{\frac{1}{2}} .
$$

Lemma 11. Under the assumptions as in Proposition 7, let $\boldsymbol{U}^{\sharp}$ be the weak solution of (3.40), (3.41). Then

$$
\left\|\boldsymbol{U}^{\sharp}\right\|_{1, \alpha, \mathcal{N}_{f}^{+}} \leq C\left(\sum_{i=1,2,3} F_{i}+\|\boldsymbol{h}\|_{1, \alpha, \mathcal{N}_{f}^{+}}+\left\|\boldsymbol{U}^{\sharp}\right\|_{H^{1}\left(\mathcal{N}_{f}^{+}\right)}+\left\|\boldsymbol{F}^{\sharp}\right\|_{L^{3}\left(\mathcal{N}_{f}^{+}\right)}\right),
$$

where $C$ is a positive constant depending on $\mu, \mathcal{M},\|A\|_{\alpha, \mathcal{N}_{f}^{+}},\|d\|_{L^{q}\left(\mathcal{N}_{f}^{+}\right)}$with $q=\frac{3}{1-\alpha}$ and $\mathcal{N}_{f}^{+}$and $F_{i}$ for $i=1,2,3$ are constants given in Lemma 11.

We will prove Lemmas 10 and 11 using the method of freezing the coefficients (Korn's device of freezing the coefficients) (see [15, Chapter 3]). Since $\mathcal{N}_{f}^{+}$is a Lipshitz domain, $\boldsymbol{U}^{\sharp} \in C^{\beta}\left(\overline{\mathcal{N}_{f}^{+}}\right)$and $\boldsymbol{U}^{\sharp} \in C^{1, \alpha}\left(\overline{\mathcal{N}_{f}^{+}}\right)$can be proved by showing that (i) there are positive constants $C$ and $R$ such that

$$
\int_{D_{t}\left(\mathrm{x}_{0}\right)}\left|D \boldsymbol{U}^{\sharp}\right|^{2} \leq C t^{3-2+2 \beta} \text { for any } 0<t<R
$$

for all $\mathrm{x}_{0} \in \overline{\mathcal{N}_{f}^{+}}$, and (ii) there are positive constants $C$ and $R$ such that

$$
\int_{D_{t}\left(\mathrm{x}_{0}\right)}\left|D \boldsymbol{U}^{\sharp}-\left(D \boldsymbol{U}^{\sharp}\right)_{\mathrm{x}_{0}, t}\right|^{2} \leq C t^{3+2 \alpha} \text { for any } 0<t<R
$$


for all $\mathrm{x}_{0} \in \overline{\mathcal{N}_{f}^{+}}$where $D_{t}\left(\mathrm{x}_{0}\right):=B_{t}\left(\mathrm{x}_{0}\right) \cap \mathcal{N}_{f}^{+}$with $B_{t}\left(\mathrm{x}_{0}\right):=\left\{\mathrm{x} \in \mathbb{R}^{3}:\left|\mathrm{x}-\mathrm{x}_{0}\right|<\right.$ $t\}$ and $\left(D \boldsymbol{U}^{\sharp}\right)_{\mathrm{x}_{0}, t}:=\frac{1}{\left|D_{t}\left(\mathrm{x}_{0}\right)\right|} \int_{D_{t}\left(\mathrm{x}_{0}\right)} D \boldsymbol{U}^{\sharp}$. We prove (i) and (ii) by obtaining (3.44) and (3.45) at each point $\mathrm{x}_{0}$ in $\overline{\mathcal{N}}_{f}^{+}$for $C$ and $R$ independent of $\mathrm{x}_{0}$ using the method of freezing the coefficients. When we do this, there exists some difficulty. For the case of $\mathrm{x}_{0} \in \mathcal{N}_{f}^{+}$or $\Gamma_{f} \cup \Gamma_{w}^{+} \cup \Gamma_{e x}$, we can obtain the integral estimates for the fixed coefficients equation using the Cacciopolli inequality and the quotient difference method, and obtain (3.44) and (3.45) at $x_{0} \in \mathcal{N}_{f}^{+}$or $\Gamma_{f} \cup \Gamma_{w}^{+} \cup \Gamma_{e x}$ using these estimates and the method of freezing the coefficients (see [1, Chapter 6]). But for the case of $\mathrm{x}_{0} \in \overline{\Gamma_{f}} \cap \overline{\Gamma_{w}^{+}}$or $\overline{\Gamma_{w}^{+}} \cap \overline{\Gamma_{e x}}$, we cannot obtain the integral estimates for the fixed coefficients equation using the Cacciopolli inequality and the quotient difference method. Thus, we cannot obtain (3.44) and (3.45) at $\mathrm{x}_{0} \in \overline{\Gamma_{f}} \cap \overline{\Gamma_{w}^{+}}$or $\overline{\Gamma_{w}^{+}} \cap \overline{\Gamma_{e x}}$ using the standard method of freezing the coefficients. We resolve this difficulty by developing some reflection argument that holds for a linear boundary value problem for an elliptic system in the case that the domain is a Lipshitz domain whose all non-differentiable parts are perpendicular corners, and the domain part of the principal coefficients of the elliptic system is diagonal with respect to the coordinate systems representing the walls near the corners of the domain. This will be seen in the proof of Lemmas 10 and 11 .

Hereafter, we use the following notation:

$\operatorname{div}_{(r, \theta, \varphi)}:=\left(\mathbf{e}_{1} \partial_{r}+\mathbf{e}_{2} \partial_{\theta}+\mathbf{e}_{3} \partial_{\varphi}\right) \cdot, \quad D_{(r, \theta, \varphi)}:=\mathbf{e}_{1} \partial_{r}+\mathbf{e}_{2} \partial_{\theta}+\mathbf{e}_{3} \partial_{\varphi}$

$\mathbb{T}:$ a one dimensional torus with period $2 \pi$,

$\mathcal{N}_{a}^{+, *}:=\left\{(r, \theta, \varphi) \in \mathbb{R}^{3} \mid a<r<r_{1}, 0<\theta<\theta_{1}, \varphi \in \mathbb{T}\right\}$,

$\Gamma_{a}^{*}:=\left\{(r, \theta, \varphi) \in \mathbb{R}^{3} \mid r=a, 0<\theta<\theta_{1}, \varphi \in \mathbb{T}\right\}$,

$\Gamma_{w, a}^{+, *}:=\left\{(r, \theta, \varphi) \in \mathbb{R}^{3} \mid a<r<r_{1}, \theta=\theta_{1}, \varphi \in \mathbb{T}\right\}$ for $0<a<r_{1}$

$\Gamma_{w}^{+, *}:=\left\{(r, \theta, \varphi) \in \mathbb{R}^{3} \mid f\left(\theta_{1}\right)<r<r_{1}, \theta=\theta_{1}, \varphi \in \mathbb{T}\right\}$,

$\Gamma_{e x}^{*}:=\left\{(r, \theta, \varphi) \in \mathbb{R}^{3} \mid r=r_{1}, 0<\theta<\theta_{1}, \varphi \in \mathbb{T}\right\}$

$\mathfrak{r}, \mathfrak{t}$ : a radius of a ball in the spherical coordinate system,

$$
\begin{aligned}
B_{\mathfrak{r}}^{*}\left(\mathrm{x}^{*}\right):= & \left\{(r, \theta, \varphi) \in \mathbb{R}^{+} \times[0, \pi] \times \mathbb{T}:\right. \\
& \left.\left|r-r^{*}\right|^{2}+\left|\theta-\theta^{*}\right|^{2}+\left|\varphi-\varphi^{*}\right|^{2}<\mathfrak{r}^{2}\right\}, \\
D_{\mathfrak{r}}^{*}\left(\mathrm{x}^{*}\right):= & B_{\mathfrak{r}}^{*}\left(\mathrm{x}^{*}\right) \cap \mathcal{N}_{f\left(\theta_{1}\right)}^{+*} \text { for } \mathrm{x}^{*}=\left(r^{*}, \theta^{*}, \varphi^{*}\right) \in \mathbb{R}^{+} \times(0, \pi) \times \mathbb{T} .
\end{aligned}
$$

To prove Lemma 10 and Lemma 11, we prove the following:

Lemma 12. Let $\mathrm{x}_{0}^{*}=\left(f\left(\theta_{1}\right), \theta_{1}, \varphi_{0}\right)$ for some $\varphi_{0} \in \mathbb{T}$. Let $0<\mathfrak{r}<\min \left(\theta_{1}, \pi\right.$, $\left.r_{1}-f\left(\theta_{1}\right)\right)$. Suppose that $\boldsymbol{W} \in H^{1}\left(D_{\mathfrak{r}}^{*}\left(\mathrm{x}_{0}^{*}\right)\right)$ is a weak solution of

$$
\begin{aligned}
& \operatorname{div}_{(r, \theta, \varphi)}\left(\left(\left.\frac{r^{2} \sin \theta}{\rho_{0}^{+}}\right|_{r=f\left(\theta_{1}\right), \theta=\theta_{1}} \mathcal{I} \otimes \mathbf{e}_{1} \otimes \mathbf{e}_{1} \otimes \mathcal{I}\right.\right. \\
& +\left.\frac{\sin \theta}{\rho_{0}^{+}}\left(\frac{c_{0}^{+2}}{c_{0}^{+^{2}}-u_{0}^{+2}}\right)\right|_{r=f\left(\theta_{1}\right), \theta=\theta_{1}} \mathcal{I} \otimes \mathbf{e}_{2} \otimes \mathbf{e}_{2} \otimes \mathcal{I}
\end{aligned}
$$




$$
\begin{aligned}
& \left.\left.\quad+\left.\frac{1}{\sin \theta \rho_{0}^{+}}\left(\frac{c_{0}^{+2}}{c_{0}^{+^{2}}-u_{0}^{+2}}\right)\right|_{r=f\left(\theta_{1}\right), \theta=\theta_{1}} \mathcal{I} \otimes \mathbf{e}_{3} \otimes \mathbf{e}_{3} \otimes \mathcal{I}\right) D_{(r, \theta, \varphi)} \boldsymbol{W}\right)=0 \\
& \text { in } D_{\mathfrak{r}}^{*}\left(\mathrm{x}_{0}^{*}\right), \\
& \boldsymbol{W}=\mathbf{0} \text { on } \partial D_{\mathfrak{r}}^{*}\left(\mathrm{x}_{0}^{*}\right) \cap\left(\overline{\Gamma_{f\left(\theta_{1}\right)}^{*}} \cup \overline{\Gamma_{w, f\left(\theta_{1}\right)}^{+, *}}\right) .
\end{aligned}
$$

Then for any $\mathfrak{t}$ such that $0<\mathfrak{t} \leq \mathfrak{r}$, there hold

$$
\int_{D_{\mathfrak{t}}^{*}\left(\mathrm{x}_{0}^{*}\right)}\left|D_{(r, \theta, \varphi)} \boldsymbol{W}\right|^{2} \leq C\left(\frac{\mathfrak{t}}{\mathfrak{r}}\right)^{3} \int_{D_{\mathfrak{r}}^{*}\left(\mathrm{x}_{0}^{*}\right)}\left|D_{(r, \theta, \varphi)} \boldsymbol{W}\right|^{2},
$$

and

$$
\begin{aligned}
& \int_{D_{\mathfrak{t}}^{*}\left(\mathrm{x}_{0}^{*}\right)}\left|D_{(r, \theta, \varphi)} \boldsymbol{W}-\left(D_{(r, \theta, \varphi)} \boldsymbol{W}\right)_{\mathrm{x}_{0}^{*}, \mathfrak{t}}^{*}\right|^{2} \\
& \leq C\left(\frac{\mathfrak{t}}{\mathfrak{r}}\right)^{5} \int_{D_{\mathfrak{r}}^{*}\left(\mathrm{x}_{0}^{*}\right)}\left|D_{(r, \theta, \varphi)} \boldsymbol{W}-\left(D_{(r, \theta, \varphi)} \boldsymbol{W}\right)_{\mathrm{x}_{0}^{*}, \mathfrak{r}}^{*}\right|^{2},
\end{aligned}
$$

where $C$ is a positive constant depending on $\mu, \mathcal{M}, f\left(\theta_{1}\right)$ and $\theta_{1}$ and $\left(D_{(r, \theta, \varphi)}\right.$ $\boldsymbol{W})_{\mathrm{x}_{0}^{*}, \mathfrak{r}}^{*}:=\frac{1}{\left|D_{\mathfrak{r}}^{*}\left(\mathrm{x}_{0}^{*}\right)\right|} \int_{D_{\mathfrak{r}}^{*}\left(\mathrm{x}_{0}^{*}\right)} D_{(r, \theta, \varphi)} \boldsymbol{W}$.

Proof. The result is obtained by using the reflection argument.

Extend (3.46) in $B_{\mathfrak{r}}^{*}\left(\mathrm{x}_{0}^{*}\right)$ :

$$
\begin{aligned}
& \operatorname{div}_{(r, \theta, \varphi)}\left(\left(\left.\frac{r^{2} \sin \theta}{\rho_{0}^{+}}\right|_{r=f\left(\theta_{1}\right), \theta=\theta_{1}} \mathcal{I} \otimes \mathbf{e}_{1} \otimes \mathbf{e}_{1} \otimes \mathcal{I}\right.\right. \\
& \quad+\left.\frac{\sin \theta}{\rho_{0}^{+}}\left(\frac{c_{0}^{+^{2}}}{c_{0}^{+^{2}}-u_{0}^{+^{2}}}\right)\right|_{r=f\left(\theta_{1}\right), \theta=\theta_{1}} \mathcal{I} \otimes \mathbf{e}_{2} \otimes \mathbf{e}_{2} \otimes \mathcal{I} \\
& \left.\left.\quad+\left.\frac{1}{\sin \theta \rho_{0}^{+}}\left(\frac{c_{0}^{+^{2}}}{c_{0}^{+^{2}}-u_{0}^{+2}}\right)\right|_{r=f\left(\theta_{1}\right), \theta=\theta_{1}} \mathcal{I} \otimes \mathbf{e}_{3} \otimes \mathbf{e}_{3} \otimes \mathcal{I}\right) D_{(r, \theta, \varphi)} \boldsymbol{W}\right)=0 \\
& \quad \text { in } B_{\mathfrak{r}}^{*}\left(\mathrm{x}_{0}^{*}\right),
\end{aligned}
$$

and extend a weak solution $\boldsymbol{W}$ of $(3.46),(3.47)$ in $B_{\mathfrak{r}}^{*}\left(x_{0}^{*}\right)$ :

$$
\boldsymbol{W}_{\text {ext }}=\left\{\begin{array}{l}
\boldsymbol{W}(r, \theta, \varphi) \text { in } B_{\mathfrak{r}}^{*}\left(\mathrm{x}_{0}^{*}\right) \cap\left\{r \geq f\left(\theta_{1}\right), \theta \leq \theta_{1}\right\} \\
-\boldsymbol{W}\left(2 f\left(\theta_{1}\right)-r, \theta, \varphi\right) \text { in } B_{\mathfrak{r}}^{*}\left(\mathrm{x}_{0}^{*}\right) \cap\left\{r<f\left(\theta_{1}\right), \theta \leq \theta_{1}\right\} \\
-\boldsymbol{W}\left(r, 2 \theta_{1}-\theta, \varphi\right) \text { in } B_{\mathfrak{r}}^{*}\left(\mathrm{x}_{0}^{*}\right) \cap\left\{r \geq f\left(\theta_{1}\right), \theta>\theta_{1}\right\} \\
\boldsymbol{W}\left(2 f\left(\theta_{1}\right)-r, 2 \theta_{1}-\theta, \varphi\right) \text { in } B_{\mathfrak{r}}^{*}\left(\mathrm{x}_{0}^{*}\right) \cap\left\{r<f\left(\theta_{1}\right), \theta>\theta_{1}\right\} .
\end{array}\right.
$$

Then $\boldsymbol{W}_{\text {ext }} \in H^{1}\left(B_{\mathfrak{r}}^{*}\left(\mathrm{x}_{0}^{*}\right)\right)$. One can see that there exists a unique weak solution of (3.50),

$$
\boldsymbol{W}=\boldsymbol{W}_{\text {ext }} \quad \text { on } \partial B_{\mathfrak{r}}^{*}\left(\mathrm{x}_{0}^{*}\right)
$$


We denote the weak solution of (3.50), (3.51) by $\overline{\boldsymbol{W}}$.

One can check that $-\overline{\boldsymbol{W}}\left(2 f\left(\theta_{1}\right)-r, \theta, \varphi\right)$ and $-\overline{\boldsymbol{W}}\left(r, 2 \theta_{1}-\theta, \varphi\right)$ are also weak solutions of (3.50), (3.51). By this fact and the uniqueness of weak solutions of (3.50), (3.51),

$$
\overline{\boldsymbol{W}}(r, \theta, \varphi)=-\overline{\boldsymbol{W}}\left(2 f\left(\theta_{1}\right)-r, \theta, \varphi\right)=-\overline{\boldsymbol{W}}\left(r, 2 \theta_{1}-\theta, \varphi\right) \text { in } \quad B_{\mathfrak{r}}^{*}\left(\mathrm{x}_{0}^{*}\right) .
$$

From (3.52), we have $\overline{\boldsymbol{W}}=0$ on $\partial D_{\mathfrak{r}}^{*}\left(\mathrm{x}_{0}^{*}\right) \cap\left(\overline{\Gamma_{f\left(\theta_{1}\right)}^{*}} \cup \overline{\Gamma_{w, f\left(\theta_{1}\right)}^{+, *}}\right)$. By this fact and the fact that $\overline{\boldsymbol{W}}$ is a unique weak solution of (3.50), (3.51), $\overline{\boldsymbol{W}}$ is a weak solution of (3.46),

$$
\overline{\boldsymbol{W}}=\left\{\begin{array}{l}
\boldsymbol{W} \text { on } \partial D_{\mathfrak{r}}^{*}\left(\mathrm{x}_{0}^{*}\right) \backslash\left(\overline{\Gamma_{f\left(\theta_{1}\right)}^{*}} \cup \overline{\Gamma_{w, f\left(\theta_{1}\right)}^{+, *}}\right) \\
0 \text { on } \partial D_{\mathfrak{r}}^{*}\left(\mathrm{x}_{0}^{*}\right) \cap\left(\overline{\Gamma_{f\left(\theta_{1}\right)}^{*}} \cup \overline{\left.\Gamma_{w, f\left(\theta_{1}\right)}^{+, *}\right)}\right.
\end{array}\right.
$$

By this fact and the uniqueness of weak solutions of (3.46) satisfying $\boldsymbol{W}=\boldsymbol{W}_{\text {ext }}$ on $\left.\partial D_{\mathfrak{r}}^{*}\left(\mathrm{x}_{0}^{*}\right) \backslash \overline{\left(\Gamma_{f\left(\theta_{1}\right)}^{*}\right.} \cup \overline{\Gamma_{w, f\left(\theta_{1}\right)}^{+, *}}\right)$ and $\boldsymbol{W}=0$ on $\partial D_{\mathfrak{r}}^{*}\left(\mathrm{x}_{0}^{*}\right) \cap\left(\overline{\Gamma_{f\left(\theta_{1}\right)}^{*}} \cup \overline{\Gamma_{w, f\left(\theta_{1}\right)}^{+, *}}\right)$, we have

$$
\bar{W}=\boldsymbol{W} \text { in } D_{\mathfrak{r}}^{*}\left(\mathrm{x}_{0}^{*}\right) .
$$

By [15, Theorem 2.1, Chapter 3], $\bar{W}$ satisfies for any $0<\mathfrak{t} \leq \mathfrak{r}$,

$$
\int_{B_{\mathfrak{t}}^{*}\left(\mathrm{x}_{0}^{*}\right)}\left|D_{(r, \theta, \varphi)} \overline{\boldsymbol{W}}\right|^{2} \leq C\left(\frac{\mathfrak{t}}{\mathfrak{r}}\right)^{3} \int_{B_{\mathfrak{r}}^{*}\left(\mathrm{x}_{0}^{*}\right)}\left|D_{(r, \theta, \varphi)} \overline{\boldsymbol{W}}\right|^{2}
$$

and

$$
\begin{aligned}
& \int_{B_{\mathfrak{t}}^{*}\left(\mathrm{x}_{0}^{*}\right)}\left|D_{(r, \theta, \varphi)} \overline{\boldsymbol{W}}-\left(D_{(r, \theta, \varphi)} \overline{\boldsymbol{W}}\right)_{\tilde{\mathrm{x}}_{0}^{*}, \mathfrak{t}}^{* *}\right|^{2} \\
& \quad \leq C\left(\frac{\mathfrak{t}}{\mathfrak{r}}\right)^{5} \int_{B_{\mathfrak{r}}^{*}\left(\mathrm{x}_{0}^{*}\right)}\left|D_{(r, \theta, \varphi)} \overline{\boldsymbol{W}}-\left(D_{(r, \theta, \varphi)} \overline{\boldsymbol{W}}\right)_{\tilde{\mathrm{x}}_{0}^{*}, \mathfrak{r}}^{* *}\right|^{2},
\end{aligned}
$$

where $C$ is a positive constant depending on $\mu, \mathcal{M}, f\left(\theta_{1}\right)$ and $\theta_{1}$ and $\left(D_{(r, \theta, \varphi)}\right.$ $\overline{\boldsymbol{W}})_{\mathrm{x}_{0}^{*}, \mathfrak{r}}^{* *}:=\frac{1}{\left|B_{\mathfrak{r}}^{*}\left(\mathrm{x}_{0}^{*}\right)\right|} \int_{B_{\mathrm{r}}^{*}\left(\mathrm{x}_{0}^{*}\right)} D_{(r, \theta, \varphi)} \overline{\boldsymbol{W}}$. By (3.52) and (3.53), we obtain from (3.54) and (3.55) for any $0<\mathfrak{t} \leq \mathfrak{r}$,

$$
4 \int_{D_{\mathfrak{t}}^{*}\left(\mathrm{x}_{0}^{*}\right)}\left|D_{(r, \theta, \varphi)} \boldsymbol{W}\right|^{2} \leq 4 C\left(\frac{\mathfrak{t}}{\mathfrak{r}}\right)^{3} \int_{D_{\mathfrak{r}}^{*}\left(\mathrm{x}_{0}^{*}\right)}\left|D_{(r, \theta, \varphi)} \boldsymbol{W}\right|^{2}
$$

and

$$
\begin{aligned}
& 4 \int_{D_{\mathfrak{t}}^{*}\left(\mathrm{x}_{0}^{*}\right)}\left|D_{(r, \theta, \varphi)} \boldsymbol{W}-\left(D_{(r, \theta, \varphi)} \boldsymbol{W}\right)_{\mathrm{x}_{0}^{*}, \mathfrak{t}}^{*}\right|^{2} \\
& \leq 4 C\left(\frac{\mathfrak{t}}{\mathfrak{r}}\right)^{5} \int_{D_{\mathfrak{r}}^{*}\left(\mathrm{x}_{0}^{*}\right)}\left|D_{(r, \theta, \varphi)} \boldsymbol{W}-\left(D_{(r, \theta, \varphi)} \boldsymbol{W}\right)_{\mathrm{x}_{0}^{*}, \mathfrak{r}}^{*}\right|^{2},
\end{aligned}
$$

where we used

$$
\left(D_{(r, \theta, \varphi)} \overline{\boldsymbol{W}}\right)_{\mathrm{x}_{0}^{*}, \mathfrak{r}}^{* *}=\frac{1}{\left|B_{\mathfrak{r}}^{*}\left(\mathrm{x}_{0}^{*}\right)\right|} \int_{B_{\mathfrak{r}}^{*}\left(\mathrm{x}_{0}^{*}\right)} D_{(r, \theta, \varphi)} \overline{\boldsymbol{W}}=\frac{1}{\left|D_{\mathfrak{r}}^{*}\left(\mathrm{x}_{0}^{*}\right)\right|} \int_{D_{\mathfrak{r}}^{*}\left(\mathrm{x}_{0}^{*}\right)} D_{(r, \theta, \varphi)} \boldsymbol{W} .
$$

This finishes the proof. 
The next corollary is obtained from Lemma 12 in the same way that Corollary 3.11 is obtained from Lemma 3.10 in [17]. We omit the proof.

Corollary 1. Suppose that $\boldsymbol{W}$ is as in Lemma 12. Let $\tilde{\boldsymbol{U}}^{*}$ be any function in $H^{1}\left(D_{\mathfrak{r}}^{*}\left(\mathrm{x}_{0}^{*}\right)\right)$ for $0<\mathfrak{r}<\min \left(\theta_{1}, \pi, r_{1}-f\left(\theta_{1}\right)\right)$. Then for any $\mathfrak{t}$ and $\mathfrak{r}$ such that $0<\mathfrak{t} \leq \mathfrak{r}<\min \left(\theta_{1}, \pi, r_{1}-f\left(\theta_{1}\right)\right)$, it holds that

$$
\begin{aligned}
& \int_{D_{\mathfrak{t}}^{*}\left(\mathrm{x}_{0}^{*}\right)}\left|D_{(r, \theta, \varphi)} \tilde{\boldsymbol{U}}^{*}\right|^{2} \\
& \leq C\left(\left(\frac{\mathfrak{t}}{\mathfrak{r}}\right)^{3} \int_{D_{\mathfrak{r}}^{*}\left(\mathrm{x}_{0}^{*}\right)}\left|D_{(r, \theta, \varphi)} \tilde{\boldsymbol{U}}^{*}\right|^{2}+\int_{D_{\mathfrak{r}}^{*}\left(\mathrm{x}_{0}^{*}\right)}\left|D_{(r, \theta, \varphi)}\left(\tilde{\boldsymbol{U}}^{*}-\mathbf{W}\right)\right|^{2}\right)
\end{aligned}
$$

and

$$
\begin{aligned}
& \int_{D_{\mathfrak{t}}^{*}\left(\mathrm{x}_{0}^{*}\right)}\left|D_{(r, \theta, \varphi)} \tilde{\boldsymbol{U}}^{*}-\left(D_{(r, \theta, \varphi)} \tilde{\boldsymbol{U}}^{*}\right)_{\mathrm{x}_{0}^{*}, \mathfrak{t}}^{*}\right|^{2} \\
& \leq C\left(\left(\frac{\mathfrak{t}}{\mathfrak{r}}\right)^{5} \int_{D_{\mathfrak{r}}^{*}\left(\mathrm{x}_{0}^{*}\right)}\left|D_{(r, \theta, \varphi)} \tilde{\boldsymbol{U}}^{*}-\left(D_{(r, \theta, \varphi)} \tilde{\boldsymbol{U}}^{*}\right)_{\mathrm{x}_{0}^{*}, \mathfrak{r}}^{*}\right|^{2}\right. \\
& \left.\quad+\int_{D_{\mathfrak{r}}^{*}\left(\mathrm{x}_{0}^{*}\right)}\left|D_{(r, \theta, \varphi)}\left(\tilde{\boldsymbol{U}}^{*}-\mathbf{W}\right)\right|^{2}\right),
\end{aligned}
$$

where $C$ is a positive constant depending on $\mu, \mathcal{M}, f\left(\theta_{1}\right)$ and $\theta_{1}$.

We first prove Lemma 10.

Proof of Lemma 10. We prove Lemma 10 by proving that (3.44) holds for all $\mathrm{x}_{0} \in \overline{\mathcal{N}_{f}^{+}}$for some positive constants $C$ and $R$.

1. Transform (3.40), (3.41) into a problem in $\mathcal{N}_{f\left(\theta_{1}\right)}^{+}$.

Define a map

$$
\Pi_{a b}^{*}(r, \theta, \varphi):=\left(\frac{r_{1}-b}{r_{1}-a}(r-a)+b, \theta, \varphi\right)
$$

for $0<a, b<r_{1}$. Then $\Pi_{a b}^{*}(r, \theta, \varphi) \operatorname{maps}\left(a, r_{1}\right) \times\left(0, \theta_{1}\right) \times \mathbb{T}$ to $\left(b, r_{1}\right) \times$ $\left(0, \theta_{1}\right) \times \mathbb{T} . \Pi_{a b}^{*}$ naturally induces a map from $\mathcal{N}_{a}^{+}$to $\mathcal{N}_{b}^{+}$as a map between two Cartesian coordinate systems. Denote this map by $\Pi_{a b}$. Using $\Pi:=\Pi_{f(\theta) f\left(\theta_{1}\right)}$, we transform the boundary value problem (3.40), (3.41) into the boundary value problem

$$
\begin{aligned}
& \operatorname{div}_{\mathrm{y}}\left(\tilde{\boldsymbol{A}} D_{\mathrm{y}} \tilde{\boldsymbol{U}}^{\sharp}\right)-\tilde{d} \tilde{\boldsymbol{U}}^{\sharp}=\tilde{\boldsymbol{F}}^{\sharp} \text { in } \mathcal{N}_{f\left(\theta_{1}\right)}^{+}, \\
& \tilde{\boldsymbol{U}}^{\sharp}=\mathbf{0} \text { on } \partial \mathcal{N}_{f\left(\theta_{1}\right)}^{+},
\end{aligned}
$$

where $\tilde{\boldsymbol{A}}:=\frac{1}{\operatorname{det}\left(\frac{\partial \Pi}{\partial \mathrm{x}}\right)}\left(\frac{\partial \Pi}{\partial \mathrm{x}}\right)^{T}\left(\boldsymbol{A} \circ \Pi^{-1}\right)\left(\frac{\partial \Pi}{\partial \mathrm{x}}\right), \tilde{d}:=\frac{d \circ \Pi^{-1}}{\operatorname{det}\left(\frac{\partial \Pi}{\partial \mathrm{x}}\right)}, \tilde{\boldsymbol{U}}^{\sharp}:=\boldsymbol{U}^{\sharp} \circ \Pi^{-1}$, $\tilde{\boldsymbol{F}}^{\sharp}:=\frac{\boldsymbol{F}^{\sharp} \circ \Pi^{-1}}{\operatorname{det}\left(\frac{\partial \Pi}{\partial \mathrm{x}}\right)}$ and $\mathrm{x}$ and $\mathrm{y}$ are the Cartesian coordinate systems for $\mathcal{N}_{f}^{+}$and $\mathcal{N}_{f\left(\theta_{1}\right)}^{+}$, respectively. 
2. Transform the weak formulation of (3.57), (3.58) near $\mathrm{x}_{0} \in \overline{\Gamma_{f\left(\theta_{1}\right)}} \cap \overline{\Gamma_{w}^{+}}$into the weak formulation of the spherical coordinate representation of (3.57), (3.58).

Write (3.57), (3.58) in the form

$$
\int_{\mathcal{N}_{f\left(\theta_{1}\right)}^{+}} \tilde{\boldsymbol{A}} D_{\mathrm{y}} \tilde{\boldsymbol{U}}^{\sharp} D_{\mathrm{y}} \boldsymbol{\xi}+\tilde{d} \tilde{\boldsymbol{U}}^{\sharp} \boldsymbol{\xi}=-\int_{\mathcal{N}_{f\left(\theta_{1}\right)}^{+}} \tilde{\boldsymbol{F}}^{\sharp} \boldsymbol{\xi}
$$

for all $\xi \in H_{0}^{1}\left(\mathcal{N}_{f\left(\theta_{1}\right)}^{+}\right)$. Let $(\tilde{r}, \tilde{\theta}, \tilde{\varphi})$ be the spherical coordinate system for $\mathrm{y}$ and $\Xi$ be the map from y to $(\tilde{r}, \tilde{\theta}, \tilde{\varphi})$. Choose any $\mathrm{x}_{0}^{*}:=\left(f\left(\theta_{1}\right), \theta_{1}, \varphi_{0}\right)$ for some $\varphi_{0} \in \mathbb{T}$ and set $\boldsymbol{\xi}=0$ outside of $\Xi^{-1}\left(D_{\mathfrak{r}}^{*}\left(\mathrm{x}_{0}^{*}\right)\right)$ for $0<\mathfrak{r}<\min \left(\theta_{1}, \pi, r_{1}-f\left(\theta_{1}\right)\right)$ in (3.59). Then we obtain

$$
\int_{\Xi^{-1}\left(D_{\mathrm{r}}^{*}\left(\mathrm{x}_{0}^{*}\right)\right)} \tilde{\boldsymbol{A}} D_{\mathrm{y}} \tilde{\boldsymbol{U}}^{\sharp} D_{\mathrm{y}} \boldsymbol{\xi}+\tilde{d} \tilde{\boldsymbol{U}}^{\sharp} \boldsymbol{\xi}=-\int_{\Xi^{-1}\left(D_{\mathrm{r}}^{*}\left(\mathrm{x}_{0}^{*}\right)\right)} \tilde{\boldsymbol{F}}^{\sharp} \boldsymbol{\xi}
$$

for all $\xi \in H_{0}^{1}\left(\Xi^{-1}\left(D_{\mathfrak{r}}^{*}\left(\mathrm{x}_{0}^{*}\right)\right)\right)$. Using $\Xi$, transform this equation. Then we have

$$
\int_{D_{\mathfrak{r}}^{*}\left(\mathrm{x}_{0}^{*}\right)} \frac{1}{\operatorname{det} \tilde{M}} \tilde{M}^{T} \tilde{\boldsymbol{A}} \circ \Xi^{-1} \tilde{M} D_{(\tilde{r}, \tilde{\theta}, \tilde{\varphi})} \tilde{\boldsymbol{U}}^{*} D_{(\tilde{r}, \tilde{\theta}, \tilde{\varphi})} \boldsymbol{\xi}+\tilde{d}^{*} \tilde{\boldsymbol{U}}^{*} \boldsymbol{\xi}=-\int_{D_{r}^{*}\left(\mathrm{x}_{0}^{*}\right)} \tilde{\boldsymbol{F}}^{*} \boldsymbol{\xi}
$$

for all $\boldsymbol{\xi} \in H_{0}^{1}\left(D_{\mathfrak{r}}^{*}\left(\mathrm{x}_{0}^{*}\right)\right)$ where $\tilde{\boldsymbol{U}}^{*}=\tilde{\boldsymbol{U}}^{\sharp} \circ \Xi^{-1}, \tilde{d}^{*}=\frac{\tilde{d} \circ \Xi^{-1}}{\operatorname{det} \tilde{M}}, \tilde{\boldsymbol{F}}^{*}=\frac{\tilde{\boldsymbol{F}}^{\sharp} \circ \Xi^{-1}}{\operatorname{det} \tilde{M}}$ and $\tilde{M}=\frac{\partial \Xi}{\partial y}$.

3. Obtain (3.44) at $\mathrm{x}_{0} \in \overline{\Gamma_{f}} \cap \overline{\Gamma_{w}^{+}}$.

Rewrite (3.60) as

$$
\begin{aligned}
& \int_{D_{\mathfrak{r}}^{*}\left(\mathrm{x}_{0}^{*}\right)} \frac{1}{\operatorname{det} \tilde{M}} \tilde{M}^{T} \boldsymbol{A} \circ \Xi^{-1} \tilde{M} D_{(\tilde{r}, \tilde{\theta}, \tilde{\varphi})} \tilde{\boldsymbol{U}}^{*} D_{(\tilde{r}, \tilde{\theta}, \tilde{\varphi})} \boldsymbol{\xi} \\
& =\int_{D_{\mathfrak{r}}^{*}\left(\mathrm{x}_{0}^{*}\right)}\left(\frac{1}{\operatorname{det} \tilde{M}} \tilde{M}^{T} \boldsymbol{A} \circ \boldsymbol{\Xi}^{-1} \tilde{M}-\frac{1}{\operatorname{det} \tilde{M}} \tilde{M}^{T} \tilde{\boldsymbol{A}} \circ \Xi^{-1} \tilde{M}\right) D_{(\tilde{r}, \tilde{\theta}, \tilde{\varphi})} \tilde{\boldsymbol{U}}^{*} D_{(\tilde{r}, \tilde{\theta}, \tilde{\varphi})} \boldsymbol{\xi} \\
& \quad-\tilde{d}^{*} \tilde{\boldsymbol{U}}^{*} \boldsymbol{\xi}-\tilde{\boldsymbol{F}}^{*} \boldsymbol{\xi}
\end{aligned}
$$

for all $\xi \in H_{0}^{1}\left(D_{\mathfrak{r}}^{*}\left(\mathrm{x}_{0}^{*}\right)\right)$. Fix the principal coefficients of the left hand side of the resultant equation at $\mathrm{x}_{0}^{*}$. Then we obtain

$$
\begin{aligned}
\int_{D_{\mathfrak{r}}^{*}\left(\mathrm{x}_{0}^{*}\right)} & \left(\frac{1}{\operatorname{det} \tilde{M}} \tilde{M}^{T} \boldsymbol{A} \circ \Xi^{-1} \tilde{M}\right)\left(\mathrm{x}_{0}^{*}\right) D_{(\tilde{r}, \tilde{\theta}, \tilde{\varphi})} \tilde{\boldsymbol{U}}^{*} D_{(\tilde{r}, \tilde{\theta}, \tilde{\varphi})} \boldsymbol{\xi} \\
= & \int_{D_{\mathfrak{r}}^{*}\left(\mathrm{x}_{0}^{*}\right)}\left(\left(\frac{1}{\operatorname{det} \tilde{M}} \tilde{M}^{T} \boldsymbol{A} \circ \Xi^{-1} \tilde{M}\right)\left(\mathrm{x}_{0}^{*}\right)-\frac{1}{\operatorname{det} \tilde{M}} \tilde{M}^{T} \boldsymbol{A} \circ \Xi^{-1} \tilde{M}\right) \\
& D_{(\tilde{r}, \tilde{\theta}, \tilde{\varphi})} \tilde{\boldsymbol{U}}^{*} D_{(\tilde{r}, \tilde{\theta}, \tilde{\varphi})} \xi \\
& +\left(\frac{1}{\operatorname{det} \tilde{M}} \tilde{M}^{T} \boldsymbol{A} \circ \Xi^{-1} \tilde{M}-\frac{1}{\operatorname{det} \tilde{M}} \tilde{M}^{T} \tilde{\boldsymbol{A}} \circ \Xi^{-1} \tilde{M}\right) D_{(\tilde{r}, \tilde{\theta}, \tilde{\varphi})} \tilde{\boldsymbol{U}}^{*} D_{(\tilde{r}, \tilde{\theta}, \tilde{\varphi})} \boldsymbol{\xi} \\
& -\tilde{d}^{*} \tilde{\boldsymbol{U}}^{*} \boldsymbol{\xi}-\tilde{\boldsymbol{F}}^{*} \boldsymbol{\xi}
\end{aligned}
$$


for all $\xi \in H_{0}^{1}\left(D_{\mathfrak{r}}^{*}\left(\tilde{\mathrm{x}}_{0}^{*}\right)\right)$. Using the argument in Appendix, it can be checked that $\left(\frac{1}{\operatorname{det} \tilde{M}} \tilde{M}^{T} \boldsymbol{A} \circ \Xi^{-1} \tilde{M}\right)\left(\mathrm{x}_{0}^{*}\right)$ is equal to the principal coefficients of the equation in (3.46).

Let $\boldsymbol{W}$ be the weak solution of

$$
\begin{aligned}
& \operatorname{div}_{(\tilde{r}, \tilde{\theta}, \tilde{\varphi})}\left(\left(\frac{1}{\operatorname{det} \tilde{M}} \tilde{M}^{T} \boldsymbol{A} \circ \boldsymbol{\Xi}^{-1} \tilde{M}\right)\left(\mathrm{x}_{0}^{*}\right) D_{(\tilde{r}, \tilde{\theta}, \tilde{\varphi})} \boldsymbol{W}\right)=0 \text { in } D_{\mathfrak{r}}^{*}\left(\mathrm{x}_{0}^{*}\right), \\
& \boldsymbol{W}=\left\{\begin{array}{l}
\left.\tilde{\boldsymbol{U}}^{*} \text { on } \partial D_{\mathfrak{r}}^{*}\left(\mathrm{x}_{0}^{*}\right) \cap \mathcal{N}_{f\left(\theta_{1}\right)}^{+, *}, \overline{\Gamma_{w, f\left(\theta_{1}\right)}^{+, *}}\right) . \\
\mathbf{0} \text { on } \partial D_{\mathfrak{r}}^{*}\left(\mathrm{x}_{0}^{*}\right) \cap \overline{\Gamma_{f\left(\theta_{1}\right)}^{*}} \cup \overline{\Gamma^{+}}
\end{array}\right.
\end{aligned}
$$

Subtract the weak formulation of (3.62), (3.63) from (3.61) and then take $\boldsymbol{\xi}=\boldsymbol{V}$ to the resultant equation. Then we obtain

$$
\begin{aligned}
& \int_{D_{\mathfrak{r}\left(\mathrm{x}_{0}^{*}\right)}}\left(\frac{1}{\operatorname{det} \tilde{M}} \tilde{M}^{T} \boldsymbol{A} \circ \Xi^{-1} \tilde{M}\right)\left(\mathrm{x}_{0}^{*}\right) D_{(\tilde{r}, \tilde{\theta}, \tilde{\varphi})} \boldsymbol{V} D_{(\tilde{r}, \tilde{\theta}, \tilde{\varphi})} \boldsymbol{V} \\
& =\int_{D_{\mathfrak{r}}^{*}\left(\mathrm{x}_{0}^{*}\right)}\left(\left(\frac{1}{\operatorname{det} \tilde{M}} \tilde{M}^{T} \boldsymbol{A} \circ \Xi^{-1} \tilde{M}\right)\left(\mathrm{x}_{0}^{*}\right)-\frac{1}{\operatorname{det} \tilde{M}} \tilde{M}^{T} \boldsymbol{A} \circ \Xi^{-1} \tilde{M}\right) D_{(\tilde{r}, \tilde{\theta}, \tilde{\varphi})} \tilde{\boldsymbol{U}}^{*} \\
& D_{(\tilde{r}, \tilde{\theta}, \tilde{\varphi})} \boldsymbol{V}+\left(\frac{1}{\operatorname{det} \tilde{M}} \tilde{M}^{T} \boldsymbol{A} \circ \Xi^{-1} \tilde{M}-\frac{1}{\operatorname{det} \tilde{M}} \tilde{M}^{T} \tilde{\boldsymbol{A}} \circ \Xi^{-1} \tilde{M}\right) \\
& D_{(\tilde{r}, \tilde{\theta}, \tilde{\varphi})} \tilde{\boldsymbol{U}}^{*} D_{(\tilde{r}, \tilde{\theta}, \tilde{\varphi})} \boldsymbol{V}-\tilde{d}^{*} \tilde{\boldsymbol{U}}^{*} \boldsymbol{V}-\tilde{\boldsymbol{F}}^{*} \boldsymbol{V},
\end{aligned}
$$

where $\boldsymbol{V}:=\tilde{\boldsymbol{U}}^{*}-\boldsymbol{W} \in H_{0}^{1}\left(D_{\mathfrak{r}}^{*}\left(\mathrm{x}_{0}^{*}\right)\right)$. Using the Sobolev and Hölder inequality, we obtain from this equation

$$
\begin{gathered}
\int_{D_{\mathfrak{r}}^{*}\left(\mathrm{x}_{0}^{*}\right)}\left|D_{(\tilde{r}, \tilde{\theta}, \tilde{\varphi})} \boldsymbol{V}\right|^{2} \leq C\left(\left(\tau_{1}^{2}(\mathfrak{r})+\tau_{2}^{2}(\mathfrak{r})\right) \int_{D_{\mathfrak{r}}^{*}\left(\mathrm{x}_{0}^{*}\right)}\left|D_{(\tilde{r}, \tilde{\theta}, \tilde{\varphi})} \tilde{\boldsymbol{U}}^{*}\right|^{2}\right. \\
\left.+\left(\int_{D_{\mathfrak{r}}^{*}\left(\mathrm{x}_{0}^{*}\right)}\left|\tilde{d}^{*}\right|^{3}\right)^{\frac{2}{3}} \int_{D_{r}^{*}\left(\mathrm{x}_{0}^{*}\right)}\left|\tilde{\boldsymbol{U}}^{*}\right|^{2}+\left(\int_{D_{\mathfrak{r}}^{*}\left(\mathrm{x}_{0}^{*}\right)}\left|\tilde{\boldsymbol{F}}^{*}\right|^{\frac{6}{5}}\right)^{\frac{5}{3}}\right),
\end{gathered}
$$

where

$$
\begin{aligned}
\tau_{1}(\mathfrak{r})= & \sup _{\substack{\mathrm{x}^{*}, \mathrm{y}^{*} \in \mathcal{N}_{f}^{+, *} \\
\left|\mathrm{x}^{*}-\mathrm{y}^{*}\right| \leq \mathfrak{r}}} \mid\left(\frac{1}{\operatorname{det} \tilde{M}} \tilde{M}^{T} \boldsymbol{A} \circ \Xi^{-1} \tilde{M}\right)\left(\mathrm{x}^{*}\right) \\
& \quad-\left(\frac{1}{\operatorname{det} \tilde{M}} \tilde{M}^{T} \boldsymbol{A} \circ \Xi^{-1} \tilde{M}\right)\left(\mathrm{y}^{*}\right) \mid
\end{aligned}
$$

and

$$
\tau_{2}(\mathfrak{r}):=\sup _{\left|\theta-\theta_{1}\right| \leq \mathfrak{r}}\left\{\left|f(\theta)-f\left(\theta_{1}\right)\right|+\left|f^{\prime}(\theta)\right|\right\}
$$

(note that $f^{\prime}\left(\theta_{1}\right)=0$ ). By Corollary 1 and (3.64), we have for any $0<\mathfrak{t} \leq \mathfrak{r}$,

$$
\int_{D_{\mathfrak{t}}^{*}\left(\mathrm{x}_{0}^{*}\right)}\left|D_{(\tilde{r}, \tilde{\theta}, \tilde{\varphi})} \tilde{\boldsymbol{U}}^{*}\right|^{2} \leq C\left(\left(\left(\frac{\mathfrak{t}}{\mathfrak{r}}\right)^{3}+\tau_{1}^{2}(\mathfrak{r})+\tau_{2}^{2}(\mathfrak{r})\right) \int_{D_{\mathfrak{r}}^{*}\left(\mathrm{x}_{0}^{*}\right)}\left|D_{(\tilde{r}, \tilde{\theta}, \tilde{\varphi})} \tilde{\boldsymbol{U}}^{*}\right|^{2}\right.
$$




$$
\left.+\left(\int_{D_{\mathfrak{r}}^{*}\left(\mathrm{x}_{0}^{*}\right)}\left|\tilde{d}^{*}\right|^{3}\right)^{\frac{2}{3}} \int_{D_{\mathfrak{r}}^{*}\left(\mathrm{x}_{0}^{*}\right)}\left|\tilde{\boldsymbol{U}}^{*}\right|^{2}+\left(\int_{D_{\mathbf{r}}^{*}\left(\mathrm{x}_{0}^{*}\right)}\left|\tilde{\boldsymbol{F}}^{*}\right|^{\frac{6}{5}}\right)^{\frac{5}{3}}\right) .
$$

Using the Hölder and Poincaré inequality, we get from this inequality

$$
\begin{gathered}
\int_{D_{\mathfrak{t}}^{*}\left(\mathrm{x}_{0}^{*}\right)}\left|D_{(\tilde{r}, \tilde{\theta}, \tilde{\varphi})} \tilde{\boldsymbol{U}}^{*}\right|^{2} \leq C\left(\left(\left(\frac{\mathfrak{t}}{\mathfrak{r}}\right)^{3}+\tau_{1}^{2}(\mathfrak{r})+\tau_{2}^{2}(\mathfrak{r})\right) \int_{D_{\mathfrak{r}}^{*}\left(\mathrm{x}_{0}^{*}\right)}\left|D_{(\tilde{r}, \tilde{\theta}, \tilde{\varphi})} \tilde{\boldsymbol{U}}^{*}\right|^{2}\right. \\
\left.+\left(\int_{D_{\mathfrak{r}}^{*}\left(\mathrm{x}_{0}^{*}\right)}\left|\tilde{d}^{*}\right|^{3}\right)^{\frac{2}{3}} \mathfrak{r}^{2} \int_{D_{\mathfrak{r}}^{*}\left(\mathrm{x}_{0}^{*}\right)}\left|D_{(\tilde{r}, \tilde{\theta}, \tilde{\varphi})} \tilde{\boldsymbol{U}}^{*}\right|^{2}+\left(\int_{D_{\mathfrak{r}}^{*}\left(\mathrm{x}_{0}^{*}\right)}\left|\tilde{\boldsymbol{F}}^{*}\right|^{p}\right)^{\frac{2}{p}} \mathfrak{r}^{3-2+2 \beta}\right),
\end{gathered}
$$

where $\beta=2-\frac{3}{p} \in(0,1)$ if $p \in\left(\frac{3}{2}, 3\right)$.

Depending on the value of $\beta$, we consider two cases.

Case 1: $3-2+2 \beta \leq 2$.

Apply Lemma 2.1 in [15, Chapter 3] to (3.65). Then we obtain that there exists $R_{1} \in\left(0, \min \left(\theta_{1}, \pi, r_{1}-f\left(\theta_{1}\right)\right)\right)$ such that for any $0<\mathfrak{r} \leq R_{1}$,

$$
\begin{aligned}
& \int_{D_{\mathfrak{r}}^{*}\left(\mathrm{x}_{0}^{*}\right)}\left|D_{(\tilde{r}, \tilde{\theta}, \tilde{\varphi})} \tilde{\boldsymbol{U}}^{*}\right|^{2} \\
& \quad \leq C \mathfrak{r}^{3-2+2 \beta}\left(\int_{D_{R_{1}}^{*}\left(\mathrm{x}_{0}^{*}\right)}\left|D_{(\tilde{r}, \tilde{\theta}, \tilde{\varphi})} \tilde{\boldsymbol{U}}^{*}\right|^{2}+\left(\int_{D_{R_{1}}^{*}\left(\mathrm{x}_{0}^{*}\right)}\left|\tilde{\boldsymbol{F}}^{*}\right|^{p}\right)^{\frac{2}{p}}\right) .
\end{aligned}
$$

Case $2: 3-2+2 \beta>2$.

As in the Case 1, we apply Lemma 2.1 in [15, Chapter 3] to (3.65). Then we obtain that there exists $R_{2} \in\left(0, \min \left(\theta_{1}, \pi, r_{1}-f\left(\theta_{1}\right)\right)\right)$ such that for any $0<\mathfrak{r} \leq R_{2}$,

$$
\int_{D_{\mathfrak{r}\left(x_{0}^{*}\right)}^{*}}\left|D_{(\tilde{r}, \tilde{\theta}, \tilde{\varphi})} \tilde{\boldsymbol{U}}^{*}\right|^{2} \leq C \mathfrak{r}^{2}\left(\int_{D_{R_{2}}^{*}\left(\mathrm{x}_{0}^{*}\right)}\left|D_{(\tilde{r}, \tilde{\theta}, \tilde{\varphi})} \tilde{\boldsymbol{U}}^{*}\right|^{2}+\left(\int_{D_{R_{2}}^{*}\left(\mathrm{x}_{0}^{*}\right)}\left|\tilde{\boldsymbol{F}}^{*}\right|^{p}\right)^{\frac{2}{p}}\right) .
$$

Substitute this into (3.65). After that, apply Lemma 2.1 in [15, Chapter 3] again. Then we obtain that there exists $R_{3} \leq R_{2}$ such that for any $0<\mathfrak{r} \leq R_{3}$, (3.66) holds with $R_{1}$ replaced by $R_{2}$.

From this result, we obtain that for any $\beta \in(0,1)$, there exists $R>0$ such that

$$
\begin{aligned}
\int_{D_{t}\left(\mathrm{x}_{0}\right)}\left|D \boldsymbol{U}^{\sharp}\right|^{2} & \leq C t^{3-2+2 \beta}\left(\left\|\boldsymbol{U}^{\sharp}\right\|_{H^{1}\left(\mathcal{N}_{f}^{+}\right)}^{2}+\left\|\boldsymbol{F}^{\sharp}\right\|_{L^{p}\left(\mathcal{N}_{f}^{+}\right)}^{2}\right) \\
& \text { for any } 0<t<R
\end{aligned}
$$

for $\mathrm{x}_{0} \in \overline{\Gamma_{f}} \cap \overline{\Gamma_{w}^{+}}$. Since $\boldsymbol{F}^{\sharp} \in C_{\left(1-\alpha, \Gamma_{w}^{+}\right)}^{\alpha}\left(\mathcal{N}_{f}^{+}\right)$for $\alpha \in\left(\frac{2}{3}, 1\right), \boldsymbol{F}^{\sharp} \in L^{3}\left(\mathcal{N}_{f}^{+}\right)$. This implies that $\boldsymbol{F}^{\sharp} \in L^{p}\left(\mathcal{N}_{f}^{+}\right)$for any $\beta \in(0,1)$ where $p=\frac{3}{2-\beta}$. Hence, we obtain (3.44) with $C$ replaced by $C_{1}=C\left(\left\|\boldsymbol{U}^{\sharp}\right\|_{H^{1}\left(\mathcal{N}_{f}^{+}\right)}^{2}+\left\|\boldsymbol{F}^{\sharp}\right\|_{L^{p}\left(\mathcal{N}_{f}^{+}\right)}^{2}\right)$ for $\mathrm{x}_{0} \in \overline{\Gamma_{f}} \cap \overline{\Gamma_{w}^{+}}$. 
4. When $\mathrm{x}_{0}$ is in $\overline{\Gamma_{w}^{+}} \cap \overline{\Gamma_{e x}}$, we obtain (3.44) with $C$ replaced by $C_{1}$ using similar arguments without the process of transforming $\mathcal{N}_{f}^{+}$into $\mathcal{N}_{f\left(\theta_{1}\right)}^{+}$. When $\mathrm{x}_{0}$ is in $\mathcal{N}_{f}^{+}$or $\Gamma_{f} \cup \Gamma_{w}^{+} \cup \Gamma_{e x}$ and far away from the corners $\overline{\Gamma_{f}} \cap \overline{\Gamma_{w}^{+}}$and $\overline{\Gamma_{w}^{+}} \cap \overline{\Gamma_{e x}}$, we obtain (3.44) with $C$ replaced by $C_{1}$ using the standard method of freezing the coefficients. When $\mathrm{x}_{0}$ is in $\mathcal{N}_{f}^{+}$or $\Gamma_{f} \cup \Gamma_{w}^{+} \cup \Gamma_{e x}$ and near $\overline{\Gamma_{f}} \cap \overline{\Gamma_{w}^{+}}$or $\overline{\Gamma_{w}^{+}} \cap \overline{\Gamma_{e x}}$, we obtain (3.44) with $C$ replaced by $C_{1}$ using the arguments in the proof of [16, Theorem 5.21] and arguments similar to the ones in Step 1-Step 3 above. Combining these results, we obtain that there exist a positive constant $R$ such that $\boldsymbol{U}^{\sharp}$ satisfies (3.44) for all $\mathrm{x}_{0} \in \overline{\mathcal{N}_{f}^{+}}$for $C=C_{1}$. This finishes the proof.

Next, we prove Lemma 11 . We prove Lemma 11 using the method of freezing the coefficients and the reflection arguments as we did in the proof of Lemma 10. When we do this, there exists some problem: since $\boldsymbol{F}^{\sharp}$ is not in $L^{p}\left(\mathcal{N}_{f}^{+}\right)$for $q=\frac{3}{1-\alpha}$ nor has the form $\operatorname{div} \boldsymbol{G}$ with $\boldsymbol{G} \in C^{\alpha}\left(\overline{\mathcal{N}_{f}^{+}}\right)$, we cannot get the required power of $t$ for the $C^{1, \alpha}$ estimate (see (3.45)) from the integral estimate of $\boldsymbol{F}^{\sharp}$ directly. We obtain this power by delivering $\theta$-derivatives imposed on some functions in $\boldsymbol{F}^{\sharp}$ to the functions multiplied to those functions in the integral form of $\boldsymbol{F}^{\sharp}$ using integration by parts and estimating the resultant integral form of $\boldsymbol{F}^{\sharp}$. To make our argument clear, we present the detailed proof.

Proof of Lemma 11. Using (3.31), write (3.40), (3.41) in the form

$$
\begin{aligned}
& \int_{\mathcal{N}_{f}^{+}} \boldsymbol{A} D \boldsymbol{U}^{\sharp} D \boldsymbol{\xi}+d \boldsymbol{U}^{\sharp} \boldsymbol{\xi} \\
& =\int_{\mathcal{N}_{f}^{+}} \sum_{i} A^{i} \partial_{r}\left(B^{i}-B^{i}\left(\mathrm{x}_{0}\right)\right) \xi_{\varphi}+\sum_{i} C^{i} \partial_{\theta}\left(D^{i}-D^{i}\left(\mathrm{x}_{0}\right)\right) \xi_{\varphi} \\
& \quad+E \frac{\partial_{\theta}(F \sin \theta)}{\sin \theta} \xi_{\varphi}+\operatorname{div}(\boldsymbol{A} D \boldsymbol{h}) \boldsymbol{\xi}-d \boldsymbol{h} \boldsymbol{\xi}
\end{aligned}
$$

for all $\boldsymbol{\xi} \in H_{0}^{1}\left(\mathcal{N}_{f}^{+}\right)$where $\xi_{\varphi}=\boldsymbol{\xi} \cdot \mathbf{e}_{\varphi}$. Using integration by parts, we change this equation into

$$
\begin{aligned}
& \int_{\mathcal{N}_{f}^{+}} \boldsymbol{A} D \boldsymbol{U}^{\sharp} D \boldsymbol{\xi}+d \boldsymbol{U}^{\sharp} \boldsymbol{\xi} \\
& =\int_{\mathcal{N}_{f}^{+}}-\sum_{i}\left(\partial_{r} A^{i}\left(B^{i}-B^{i}\left(\mathrm{x}_{0}\right)\right) \xi_{\varphi}+A^{i}\left(B^{i}-B^{i}\left(\mathrm{x}_{0}\right)\right) \frac{1}{r^{2}} \partial_{r}\left(r^{2} \xi_{\varphi}\right)\right) \\
& \quad-\sum_{i}\left(\partial_{\theta} C^{i}\left(D^{i}-D^{i}\left(\mathrm{x}_{0}\right)\right) \xi_{\varphi}+C^{i}\left(D^{i}-D^{i}\left(\mathrm{x}_{0}\right)\right) \frac{1}{\sin \theta} \partial_{\theta}\left(\xi_{\varphi} \sin \theta\right)\right) \\
& \quad+E \frac{\partial_{\theta}(F \sin \theta)}{\sin \theta} \xi_{\varphi}-\boldsymbol{A} D \boldsymbol{h} D \xi-d \boldsymbol{h} \boldsymbol{\xi}
\end{aligned}
$$

for all $\xi \in H_{0}^{1}\left(\mathcal{N}_{f}^{+}\right)$. Using $\Pi$ defined in Step 1 in the proof of Lemma 10, transform this equation. Then we obtain

$$
\int_{\mathcal{N}_{f\left(\theta_{1}\right)}^{+}} \tilde{\boldsymbol{A}} D \tilde{\boldsymbol{U}}^{\sharp} D \boldsymbol{\xi}+\tilde{d} \tilde{\boldsymbol{U}}^{\sharp} \boldsymbol{\xi}=\int_{\mathcal{N}_{f\left(\theta_{1}\right)}^{+}}(a) \frac{1}{\operatorname{det}\left(\frac{d \mathrm{y}}{d \mathrm{x}}\right)}-\tilde{\boldsymbol{A}} D \tilde{\boldsymbol{h}} D \boldsymbol{\xi}-\tilde{d} \tilde{\boldsymbol{h}} \boldsymbol{\xi}
$$


for all $\xi \in H_{0}^{1}\left(\mathcal{N}_{f\left(\theta_{1}\right)}^{+}\right)$where

$$
\begin{aligned}
(a)= & -\sum_{i}\left(\frac{\partial \tilde{r}}{\partial r} \partial_{\tilde{r}} \tilde{A}^{i}\left(\tilde{B}^{i}-B^{i}\left(\mathrm{x}_{0}\right)\right) \xi_{\varphi}+\tilde{A}^{i}\left(\tilde{B}^{i}-B^{i}\left(\mathrm{x}_{0}\right)\right) \frac{1}{r^{2}} \frac{\partial \tilde{r}}{\partial r} \partial_{\tilde{r}}\left(r^{2} \xi_{\varphi}\right)\right) \\
& -\sum_{i}\left(\left(\frac{\partial \tilde{r}}{\partial \theta} \partial_{\tilde{r}}+\partial_{\tilde{\theta}}\right) \tilde{C}^{i}\left(\tilde{D}^{i}-D^{i}\left(\mathrm{x}_{0}\right)\right) \xi_{\varphi}+\tilde{C}^{i}\left(\tilde{D}^{i}-D^{i}\left(\mathrm{x}_{0}\right)\right)\right. \\
& \left.\frac{1}{\sin \tilde{\theta}}\left(\frac{\partial \tilde{r}}{\partial \theta} \partial_{\tilde{r}}+\partial_{\tilde{\theta}}\right)\left(\xi_{\varphi} \sin \tilde{\theta}\right)\right)+\tilde{E} \frac{1}{\sin \tilde{\theta}}\left(\frac{\partial \tilde{r}}{\partial \theta} \partial_{\tilde{r}}+\partial_{\tilde{\theta}}\right)(\tilde{F} \sin \tilde{\theta}) \xi_{\varphi},
\end{aligned}
$$

$\tilde{\boldsymbol{A}}, \tilde{d}$ and $\tilde{\boldsymbol{U}}^{\sharp}$ are functions given below (3.58),

$$
\begin{aligned}
& \tilde{A}^{i}:=A^{i} \circ \Pi^{-1}, \tilde{B}^{i}:=B^{i} \circ \Pi^{-1}, \tilde{C}^{i}:=C^{i} \circ \Pi^{-1}, \\
& \tilde{D}^{i}=D^{i} \circ \Pi^{-1}, \tilde{E}:=E \circ \Pi^{-1}, \tilde{F}:=F \circ \Pi^{-1}, \tilde{\boldsymbol{h}}=\boldsymbol{h} \circ \Pi^{-1},
\end{aligned}
$$

$\mathrm{x}$ is the Cartesian coordinate representing $\mathcal{N}_{f}^{+}, \mathrm{y}=\Pi(\mathrm{x})$ and $(r, \theta, \varphi)$ and $(\tilde{r}, \tilde{\theta}, \tilde{\varphi})$ are the spherical coordinate systems for $\mathrm{x}$ and $\mathrm{y}$, respectively. As we did in Step 2 in the proof of Lemma 10 , set $\boldsymbol{\xi}=0$ outside of $\Xi^{-1}\left(D_{\mathfrak{r}}^{*}\left(\mathrm{x}_{0}^{*}\right)\right)$ for $0<\mathfrak{r}<$ $\min \left(\theta_{1}, \pi, r_{1}-f\left(\theta_{1}\right)\right)$ where $\mathrm{x}_{0}^{*}=\left(f\left(\theta_{1}\right), \theta_{1}, \varphi_{0}\right)$ for some $\varphi_{0} \in \mathbb{T}$ and then transform this equation using $\Xi$. Then we obtain

$$
\begin{aligned}
\int_{D_{\mathfrak{r}}^{*}\left(\mathrm{x}_{0}^{*}\right)} & \frac{1}{\operatorname{det} \tilde{M}} \tilde{M}^{T} \tilde{\boldsymbol{A}} \circ \Xi^{-1} \tilde{M} D_{(\tilde{r}, \tilde{\theta}, \tilde{\varphi})} \tilde{\boldsymbol{U}}^{*} D_{(\tilde{r}, \tilde{\theta}, \tilde{\varphi})} \boldsymbol{\xi}+\tilde{d}^{*} \tilde{\boldsymbol{U}}^{*} \boldsymbol{\xi} \\
= & \int_{D_{\mathfrak{r}}^{*}\left(\mathrm{x}_{0}^{*}\right)}(a) \frac{\tilde{r}^{2} \sin \tilde{\theta}}{\operatorname{det}\left(\frac{d \mathrm{y}}{d \mathrm{x}}\right)}-\left(\frac{1}{\operatorname{det} \tilde{M}} \tilde{M}^{T} \tilde{\boldsymbol{A}} \circ \Xi^{-1} \tilde{M} D_{(\tilde{r}, \tilde{\theta}, \tilde{\varphi})} \tilde{\boldsymbol{h}}^{*}\right. \\
& \left.-\left(\frac{1}{\operatorname{det} \tilde{M}} \tilde{M}^{T} \tilde{\boldsymbol{A}} \circ \Xi^{-1} \tilde{M} D_{(\tilde{r}, \tilde{\theta}, \tilde{\varphi})} \tilde{\boldsymbol{h}}^{*}\right)\left(\mathrm{x}_{0}^{*}\right)\right) D_{(\tilde{r}, \tilde{\theta}, \tilde{\varphi})} \boldsymbol{\xi}-\tilde{d}^{*} \tilde{\boldsymbol{h}}^{*} \boldsymbol{\xi}
\end{aligned}
$$

for all $\boldsymbol{\xi} \in H_{0}^{1}\left(D_{\mathfrak{r}}^{*}\left(\mathrm{x}_{0}^{*}\right)\right)$, where $\tilde{\boldsymbol{U}}^{*}, \tilde{d}^{*}$, and $\tilde{M}$ are functions defined below (3.60) and we used $\operatorname{div}_{(\tilde{r}, \tilde{\theta}, \tilde{\varphi})}\left(\left(\frac{1}{\operatorname{det} \tilde{M}} \tilde{M}^{T} \tilde{\boldsymbol{A}} \circ \boldsymbol{\Xi}^{-1} \tilde{M} D_{(\tilde{r}, \tilde{\theta}, \tilde{\varphi})} \tilde{\boldsymbol{h}}^{*}\right)\left(\mathrm{x}_{0}^{*}\right)\right)=0$. Fix the principal coefficients of the left hand side of the above equation. Then we get

$$
\begin{aligned}
& \int_{D_{\mathrm{r}}^{*}\left(\mathrm{x}_{0}^{*}\right)}\left(\frac{1}{\operatorname{det} \tilde{M}} \tilde{M}^{T} \boldsymbol{A} \circ \Xi^{-1} \tilde{M}\right)\left(\mathrm{x}_{0}^{*}\right) D_{(\tilde{r}, \tilde{\theta}, \tilde{\varphi})} \tilde{\boldsymbol{U}}^{*} D_{(\tilde{r}, \tilde{\theta}, \tilde{\varphi})} \xi \\
& =\int_{D_{\mathfrak{r}}^{*}\left(\mathrm{x}_{0}^{*}\right)}\left(\left(\frac{1}{\operatorname{det} \tilde{M}} \tilde{M}^{T} \boldsymbol{A} \circ \Xi^{-1} \tilde{M}\right)\left(\mathrm{x}_{0}^{*}\right)-\frac{1}{\operatorname{det} \tilde{M}} \tilde{M}^{T} \boldsymbol{A} \circ \Xi^{-1} \tilde{M}\right) D_{(\tilde{r}, \tilde{\theta}, \tilde{\varphi})} \tilde{\boldsymbol{U}}^{*} \\
& D_{(\tilde{r}, \tilde{\theta}, \tilde{\varphi})} \xi+\left(\frac{1}{\operatorname{det} \tilde{M}} \tilde{M}^{T} \boldsymbol{A} \circ \Xi^{-1} \tilde{M}-\frac{1}{\operatorname{det} \tilde{M}} \tilde{M}^{T} \tilde{\boldsymbol{A}} \circ \Xi^{-1} \tilde{M}\right) D_{(\tilde{r}, \tilde{\theta}, \tilde{\varphi})} \tilde{\boldsymbol{U}}^{*} \\
& D_{(\tilde{r}, \tilde{\theta}, \tilde{\varphi})} \xi-\tilde{d}^{*} \tilde{\boldsymbol{U}}^{*} \xi+(a) \frac{\tilde{r}^{2} \sin \tilde{\theta}}{\operatorname{det}\left(\frac{d \mathrm{y}}{d \mathrm{x}}\right)}-\left(\frac{1}{\operatorname{det} \tilde{M}} \tilde{M}^{T} \tilde{\boldsymbol{A}} \circ \Xi^{-1} \tilde{M} D_{(\tilde{r}, \tilde{\theta}, \tilde{\varphi})} \tilde{\boldsymbol{h}}^{*}\right. \\
& \left.\quad-\left(\frac{1}{\operatorname{det} \tilde{M}} \tilde{M}^{T} \tilde{\boldsymbol{A}} \circ \Xi^{-1} \tilde{M} D_{(\tilde{r}, \tilde{\theta}, \tilde{\varphi})} \tilde{\boldsymbol{h}}^{*}\right)\left(\mathrm{x}_{0}^{*}\right)\right) D_{(\tilde{r}, \tilde{\theta}, \tilde{\varphi})} \boldsymbol{\xi}-\tilde{d}^{*} \tilde{\boldsymbol{h}}^{*} \boldsymbol{\xi}
\end{aligned}
$$

for all $\xi \in H_{0}^{1}\left(D_{\mathfrak{r}}^{*}\left(\mathrm{x}_{0}^{*}\right)\right)$. 
Let $\boldsymbol{W}$ be the weak solution of (3.62), (3.63). As we did in Step 3 in the proof of Lemma 10, subtracting the weak formulation of (3.62), (3.63) from the above equation. And then take $\boldsymbol{\xi}=\boldsymbol{V}$ where $\boldsymbol{V}=\tilde{\boldsymbol{U}}^{*}-\boldsymbol{W}$ to the resultant equation. Then we have

$$
\begin{aligned}
& \int_{D_{\mathfrak{r}}^{*}\left(\mathrm{x}_{0}^{*}\right)}\left(\frac{1}{\operatorname{det} \tilde{M}} \tilde{M}^{T} \boldsymbol{A} \circ \Xi^{-1} \tilde{M}\right)\left(\mathrm{x}_{0}^{*}\right) D_{(\tilde{r}, \tilde{\theta}, \tilde{\varphi})} \boldsymbol{V} D_{(\tilde{r}, \tilde{\theta}, \tilde{\varphi})} \boldsymbol{V} \\
& =\int_{D_{\mathfrak{r}}^{*}\left(\mathrm{x}_{0}^{*}\right)}\left(\left(\frac{1}{\operatorname{det} \tilde{M}} \tilde{M}^{T} \boldsymbol{A} \circ \Xi^{-1} \tilde{M}\right)\left(\mathrm{x}_{0}^{*}\right)-\frac{1}{\operatorname{det} \tilde{M}} \tilde{M}^{T} \boldsymbol{A} \circ \Xi^{-1} \tilde{M}\right) D_{(\tilde{r}, \tilde{\theta}, \tilde{\varphi})} \tilde{\boldsymbol{U}}^{*} \\
& D_{(\tilde{r}, \tilde{\theta}, \tilde{\varphi})} \boldsymbol{V}+\left(\frac{1}{\operatorname{det} \tilde{M}} \tilde{M}^{T} \boldsymbol{A} \circ \Xi^{-1} \tilde{M}-\frac{1}{\operatorname{det} \tilde{M}} \tilde{M}^{T} \tilde{\boldsymbol{A}} \circ \Xi^{-1} \tilde{M}\right) D_{(\tilde{r}, \tilde{\theta}, \tilde{\varphi})} \tilde{\boldsymbol{U}}^{*} \\
& D_{(\tilde{r}, \tilde{\theta}, \tilde{\varphi})} \boldsymbol{V}-\tilde{d}^{*} \tilde{\boldsymbol{U}}^{*} \boldsymbol{V}+\left(a^{\prime}\right) \frac{\tilde{r}^{2} \sin \tilde{\theta}}{\operatorname{det}\left(\frac{d \mathrm{y}}{d \mathrm{x}}\right)}-\left(\frac{1}{\operatorname{det} \tilde{M}} \tilde{M}^{T} \tilde{\boldsymbol{A}} \circ \Xi^{-1} \tilde{M} D_{(\tilde{r}, \tilde{\theta}, \tilde{\varphi})} \tilde{\boldsymbol{h}}^{*}\right. \\
& \left.\quad-\left(\frac{1}{\operatorname{det} \tilde{M}} \tilde{M}^{T} \tilde{\boldsymbol{A}} \circ \Xi^{-1} \tilde{M} D_{(\tilde{r}, \tilde{\theta}, \tilde{\varphi})} \tilde{\boldsymbol{h}}^{*}\right)\left(\mathrm{x}_{0}^{*}\right)\right) D_{(\tilde{r}, \tilde{\theta}, \tilde{\varphi})} \boldsymbol{V}-\tilde{d}^{*} \tilde{\boldsymbol{h}}^{*} \boldsymbol{V},
\end{aligned}
$$

where $V_{\varphi}=\boldsymbol{V} \cdot \mathbf{e}_{\varphi}$ and $\left(a^{\prime}\right)$ is $(a)$ with $\xi_{\varphi}$ replaced by $V_{\varphi}$. Using the Sobolev and Hölder inequality and the facts that $\boldsymbol{A} \in C^{\alpha}\left(\overline{\mathcal{N}_{f}^{+}}\right), f \in C^{1, \alpha}(\bar{\Lambda}), \boldsymbol{h} \in C^{1, \alpha}\left(\overline{\mathcal{N}_{f}^{+}}\right)$ and

$$
\begin{aligned}
& \int_{D_{\mathfrak{r}}^{*}\left(\mathrm{x}_{0}^{*}\right)}\left|\frac{1}{r^{2}} \partial_{\tilde{r}}\left(r^{2} V_{\varphi}\right)\right|^{2}, \int_{D_{\mathfrak{r}}^{*}\left(\mathrm{x}_{0}^{*}\right)}\left|\frac{1}{\sin \tilde{\theta}} \partial_{\tilde{\theta}}\left(V_{\varphi} \sin \tilde{\theta}\right)\right|^{2} \\
& \leq C \int_{D_{\mathfrak{r}}^{*}\left(\mathrm{x}_{0}^{*}\right)}\left|D\left(V_{\varphi} \mathbf{e}_{\varphi}\right)\right|^{2}(\operatorname{See}(5.7)) \\
& \leq C \int_{D_{\mathfrak{r}}^{*}\left(\mathrm{x}_{0}^{*}\right)}\left|D_{(\tilde{r}, \tilde{\theta}, \tilde{\varphi})} \boldsymbol{V}\right|^{2}
\end{aligned}
$$

we obtain from the above equation

$$
\begin{gathered}
\int_{D_{\mathfrak{r}}^{*}\left(\mathrm{x}_{0}^{*}\right)}\left|D_{(r, \theta, \varphi)} \boldsymbol{V}\right|^{2} \leq C\left(\mathfrak{r}^{2 \alpha} \int_{D_{r}^{*}\left(\mathrm{x}_{0}^{*}\right)}\left|D_{(r, \theta, \varphi)} \tilde{\boldsymbol{U}}^{*}\right|^{2}\right. \\
\left.+\left(\int_{D_{\mathfrak{r}}^{*}\left(\mathrm{x}_{0}^{*}\right)}\left|\tilde{d}^{*}\right|^{q}\right)^{\frac{2}{q}} \mathfrak{r}^{2 \alpha} \int_{D_{\mathfrak{r}}^{*}\left(\mathrm{x}_{0}^{*}\right)}\left|\tilde{\boldsymbol{U}}^{*}\right|^{2}+\mathfrak{r}^{3+2 \alpha} F^{b}\right)
\end{gathered}
$$

for $q=\frac{3}{1-\alpha}$, where

$$
\begin{aligned}
F^{b}= & \sum_{i}\left\|B^{i} \mathbf{e}_{\theta}\right\|_{\alpha, \mathcal{N}_{f}^{+}}^{2}\left\|A^{i}\right\|_{W^{1,3}\left(\mathcal{N}_{f}^{+}\right) \cap L^{\infty}\left(\mathcal{N}_{f}^{+}\right)}^{2} \\
& +\sum_{i}\left\|D^{i}\right\|_{\alpha, \mathcal{N}_{f}^{+}}^{2}\left\|C^{i}\right\|_{W^{1,3}\left(\mathcal{N}_{f}^{+}\right) \cap L^{\infty}\left(\mathcal{N}_{f}^{+}\right)}^{2} \\
& +\left\|E \frac{\partial_{\theta}(F \sin \theta)}{\sin \theta} \mathbf{e}_{\varphi}\right\|_{L^{q}\left(\mathcal{N}_{f}^{+}\right)}^{2}+\|\boldsymbol{h}\|_{1, \alpha, \mathcal{N}_{f}^{+}}^{2}
\end{aligned}
$$


with $\|\cdot\|_{W^{1,3}(\Omega) \cap L^{\infty}(\Omega)}:=\|\cdot\|_{W^{1,3}(\Omega)}+\|\cdot\|_{L^{\infty}(\Omega)}$. By Corollory 1 and (3.67), we have, for any $0<\mathfrak{t} \leq \mathfrak{r}$,

$$
\begin{aligned}
& \int_{D_{\mathfrak{t}}^{*}\left(\mathrm{x}_{0}^{*}\right)}\left|D_{(\tilde{r}, \tilde{\theta}, \tilde{\varphi})} \tilde{\boldsymbol{U}}^{*}-\left(D_{(\tilde{r}, \tilde{\theta}, \tilde{\varphi})} \tilde{\boldsymbol{U}}^{*}\right)_{\mathrm{x}_{0}^{*}, \mathrm{t}}^{*}\right|^{2} \\
& \leq C\left(\left(\begin{array}{c}
\mathfrak{t} \\
\mathfrak{r}
\end{array}\right)^{5} \int_{D_{\mathfrak{r}}^{*}\left(\tilde{x}_{0}\right)}\left|D_{(\tilde{r}, \tilde{\theta}, \tilde{\varphi})} \tilde{\boldsymbol{U}}^{*}-\left(D_{(\tilde{r}, \tilde{\theta}, \tilde{\varphi})} \tilde{\boldsymbol{U}}^{*}\right)_{\mathrm{x}_{0}^{*}, \mathfrak{r}}^{*}\right|^{2}\right. \\
& \left.+\mathfrak{r}^{2 \alpha} \int_{D_{\mathfrak{r}}^{*}\left(\mathrm{x}_{0}^{*}\right)}\left|D_{(\tilde{r}, \tilde{\theta}, \tilde{\varphi})} \tilde{\boldsymbol{U}}^{*}\right|^{2}+\mathfrak{r}^{2 \alpha} \int_{D_{\mathfrak{r}}^{*}\left(\mathrm{x}_{0}^{*}\right)}\left|\tilde{\boldsymbol{U}}^{*}\right|^{2}+\mathfrak{r}^{3+2 \alpha} F^{b}\right) .
\end{aligned}
$$

In the proof of Lemma 10 , we showed that for any $\varepsilon \in(0,1)$, there exists $R_{4}>0$ such that

$$
\begin{aligned}
& \int_{D_{\mathfrak{r}}^{*}\left(\mathrm{x}_{0}^{*}\right)}\left|D_{(\tilde{r}, \tilde{\theta}, \tilde{\varphi})} \tilde{\boldsymbol{U}}^{*}\right|^{2} \leq C \mathfrak{r}^{3-2 \varepsilon}\left(\left\|\boldsymbol{U}^{\sharp}\right\|_{H^{1}\left(\mathcal{N}_{f}^{+}\right)}^{2}+\left\|\boldsymbol{F}^{\sharp}\right\|_{L^{3}\left(\mathcal{N}_{f}^{+}\right)}^{2}\right) \\
& \quad \text { for any } 0<\mathfrak{r} \leq R_{4} .
\end{aligned}
$$

Using this inequality and $\boldsymbol{U}^{\sharp} \in C^{0}\left(\overline{\mathcal{N}_{f}^{+}}\right)$obtained from Lemma 10, we apply Lemma 2.1 in [15, Chapter 3] to (3.68). Then we obtain

$$
\begin{aligned}
& \int_{D_{\mathfrak{r}}^{*}\left(\mathrm{x}_{0}^{*}\right)}\left|D_{(\tilde{r}, \tilde{\theta}, \tilde{\varphi})} \tilde{\boldsymbol{U}}^{*}-\left(D_{(\tilde{r}, \tilde{\theta}, \tilde{\varphi})} \tilde{\boldsymbol{U}}^{*}\right)_{\mathrm{x}_{0}^{*}, \mathfrak{r}}^{*}\right|^{2} \\
& \leq C\left(\left\|\boldsymbol{U}^{\sharp}\right\|_{H^{1}\left(\mathcal{N}_{f}^{+}\right)}^{2}+\left\|\boldsymbol{F}^{\sharp}\right\|_{L^{3}\left(\mathcal{N}_{f}^{+}\right)}^{2}+F^{b}\right) \mathfrak{r}^{3+2 \alpha-2 \varepsilon} \\
& \quad \text { for any } 0<\mathfrak{r}<R_{5}
\end{aligned}
$$

for a constant $R_{5}>0$.

When $\mathrm{x}_{0}^{*}$ is in $\overline{\Gamma_{w}^{+* *}} \cap \overline{\Gamma_{e x}^{*}}$, we obtain (3.69) using similar argument without the process of transforming $\mathcal{N}_{f}^{+}$to $\mathcal{N}_{f\left(\theta_{1}\right)}^{+}$. When $x_{0}^{*}$ is in $\mathcal{N}_{f}^{+, *} \cap\left\{\theta \geq \frac{\theta_{1}}{3}\right\}$ or $\left(\Gamma_{f}^{*} \cup \Gamma_{w}^{+, *} \cup \Gamma_{e x}^{*}\right) \cap\left\{\theta \geq \frac{\theta_{1}}{3}\right\}$ and far away from the corners $\overline{\Gamma_{f}^{*}} \cap \overline{\Gamma_{w}^{+, *}}$ and $\overline{\Gamma_{w}^{+, *}} \cap \overline{\Gamma_{e x}^{*}}$, we obtain (3.69) using the standard method of freezing coefficients to the spherical coordinate representation of (3.40), (3.41) with integration by parts argument above. When $\mathrm{x}_{0}^{*}$ is in $\mathcal{N}_{f}^{+, *} \cap\left\{\theta \geq \frac{\theta_{1}}{3}\right\}$ or $\Gamma_{f}^{*} \cup \Gamma_{w}^{+, *} \cup \Gamma_{e x}^{*} \cap\{\theta \geq$ $\left.\frac{\theta_{1}}{3}\right\}$ and near $\overline{\Gamma_{f}^{*}} \cap \overline{\Gamma_{w}^{+*}}$ or $\overline{\Gamma_{w}^{+, *}} \cap \overline{\Gamma_{e x}^{*}}$, we obtain (3.69) using the arguments in [16, Theorem 5.21] and arguments similar to the ones above. Then we obtain $D \boldsymbol{U}^{\sharp} \in C^{\alpha-\varepsilon}\left(\left[f(\theta), r_{1}\right] \times\left[\frac{\theta_{1}}{3}, \theta_{1}\right] \times \mathbb{T}\right)$. When $\mathrm{x}_{0}$ is in $\mathcal{N}_{f}^{+} \cap\left\{\theta \leq \frac{2 \theta_{1}}{3}\right\}$ or $\left(\Gamma_{f} \cup \Gamma_{w}^{+} \cup \Gamma_{e x}\right) \cap\left\{\theta \leq \frac{2 \theta_{1}}{3}\right\}$, we obtain (3.45) with $\alpha$ and $C$ replaced by $\alpha-\varepsilon$ for any $\varepsilon \in(0,1)$ and $C\left(\left\|\boldsymbol{U}^{\sharp}\right\|_{H^{1}\left(\mathcal{N}_{f}^{+}\right)}^{2}+\left\|\boldsymbol{F}^{\sharp}\right\|_{L^{3}\left(\mathcal{N}_{f}^{+}\right)}^{2}+F^{b}\right)$ using the standard method of freezing coefficients to (3.40), (3.41) with integration by parts argument above. Note that if we estimate the integral form of $\boldsymbol{F}^{\sharp}$ using integration by parts argument above, then there is no singularity issue. From this result, we obtain $D \boldsymbol{U}^{\sharp} \in C^{\alpha-\varepsilon}\left(\overline{\mathcal{N}_{f}^{+}} \cap\left\{\theta \leq \frac{2 \theta_{1}}{3}\right\}\right)$. Combining these two regularity results for $D \boldsymbol{U}^{\sharp}$, we obtain $D \boldsymbol{U}^{\sharp} \in C^{\alpha-\varepsilon}\left(\overline{\mathcal{N}_{f}^{+}}\right)$. 
Using the regularity result for $D \boldsymbol{U}^{\sharp}$ and $\boldsymbol{U}^{\sharp} \in C^{0}\left(\overline{\mathcal{N}_{f}^{+}}\right)$, we obtain from (3.68)

$$
\begin{aligned}
& \int_{D_{\mathfrak{t}}^{*}\left(\mathrm{x}_{0}^{*}\right)}\left|D_{(\tilde{r}, \tilde{\theta}, \tilde{\varphi})} \tilde{\boldsymbol{U}}^{*}-\left(D_{(\tilde{r}, \tilde{\theta}, \tilde{\varphi})} \tilde{\boldsymbol{U}}^{*}\right)_{\mathrm{x}_{0}^{*}, \mathfrak{t}}^{*}\right|^{2} \\
& \leq C\left(\left(\frac{\mathfrak{t}}{\mathfrak{r}}\right)^{5} \int_{D_{\mathfrak{r}\left(\mathrm{x}_{0}^{*}\right)}}\left|D_{(\tilde{r}, \tilde{\theta}, \tilde{\varphi})} \tilde{\boldsymbol{U}}^{*}-\left(D_{(\tilde{r}, \tilde{\theta}, \tilde{\varphi})} \tilde{\boldsymbol{U}}^{*}\right)_{\mathrm{x}_{0}^{*}, \mathfrak{r}}^{*}\right|^{2}\right. \\
& \left.\quad+\mathfrak{r}^{3+2 \alpha}\left(\left\|\boldsymbol{U}^{\sharp}\right\|_{H^{1}\left(\mathcal{N}_{f}^{+}\right)}^{2}+\left\|\boldsymbol{F}^{\sharp}\right\|_{L^{3}\left(\mathcal{N}_{f}^{+}\right)}^{2}+F^{b}\right)\right)
\end{aligned}
$$

(here $\mathrm{x}_{0}^{*} \in \overline{\Gamma_{f}^{*}} \cap \overline{\Gamma_{w}^{+, *}}$ ). Apply Lemma 2.1 in [15, Chapter 3] to (3.70). Then we have

$$
\begin{aligned}
& \int_{D_{\mathfrak{r}}^{*}\left(\mathrm{x}_{0}^{*}\right)}\left|D_{(\tilde{r}, \tilde{\theta}, \tilde{\varphi})} \tilde{\boldsymbol{U}}^{*}-\left(D_{(\tilde{r}, \tilde{\theta}, \tilde{\varphi})} \tilde{\boldsymbol{U}}^{*}\right)_{\mathrm{x}_{0}^{*}, \mathfrak{r}}^{*}\right|^{2} \\
& \leq C\left(\left\|\boldsymbol{U}^{\sharp}\right\|_{H^{1}\left(\mathcal{N}_{f}^{+}\right)}^{2}+\left\|\boldsymbol{F}^{\sharp}\right\|_{L^{3}\left(\mathcal{N}_{f}^{+}\right)}^{2}+F^{b}\right) \mathfrak{r}^{3+2 \alpha} \\
& \quad \text { for any } 0<\mathfrak{r}<R_{6}
\end{aligned}
$$

for a constant $R_{6}>0$. Using the arguments used when we obtained $D \boldsymbol{U} \in$ $C^{\alpha-\varepsilon}\left(\overline{\mathcal{N}_{f}^{+}}\right)$, we obtain (3.71) at $\mathrm{x}_{0}^{*} \in \overline{\mathcal{N}_{f}^{+, *}} \cap\left\{\theta \geq \frac{\theta_{1}}{3}\right\}$ and (3.45) with $C$ replaced by $C\left(\left\|\boldsymbol{U}^{\sharp}\right\|_{H^{1}\left(\mathcal{N}_{f}^{+}\right)}^{2}+\left\|\boldsymbol{F}^{\sharp}\right\|_{L^{3}\left(\mathcal{N}_{f}^{+}\right)}^{2}+F^{b}\right)$ at $\mathrm{x}_{0} \in \overline{\mathcal{N}_{f}^{+}} \cap\left\{\theta \leq \frac{2 \theta_{1}}{3}\right\}$. From this result, we obtain the desired result. This finishes the proof.

Using the scailing argument given in the proof of Proposition 3.1 in [2] with the results in Theorem 5.21 in [16] and Lemma 11, we can obtain that the weak solution of (3.40), (3.41) is in $C_{\left(-1-\alpha, \Gamma_{w}^{+}\right)}^{2, \alpha}\left(\mathcal{N}_{f}^{+}\right)$and satisfies the estimate in the following lemma (we omit the proof):

Lemma 13. Under the assumption as in Lemma 7, let $\boldsymbol{U}^{\sharp}$ be the weak solution of (3.40), (3.41). Then $\boldsymbol{U}^{\sharp} \in C_{\left(-1-\alpha, \Gamma_{w}^{+}\right)}^{2, \alpha}\left(\mathcal{N}_{f}^{+}\right)$. Furthermore, $\boldsymbol{U}^{\sharp}$ satisfies

$$
\left\|\boldsymbol{U}^{\sharp}\right\|_{2, \alpha, \mathcal{N}_{f}^{+}}^{\left(-1-\alpha, \Gamma_{w}^{+}\right)} \leq C\left(\left\|\boldsymbol{F}^{\sharp}\right\|_{\alpha, \mathcal{N}_{f}^{+}}^{\left(1-\alpha, \Gamma_{w}^{+}\right)}+\left\|\boldsymbol{U}^{\sharp}\right\|_{1, \alpha, \mathcal{N}_{f}^{+}}\right),
$$

where $C$ is a positive constant depending on $\left(\rho_{0}^{+}, u_{0}^{+}, p_{0}^{+}\right), \gamma, \mathcal{N}_{f}^{+}$and $\alpha$.

Finally, we prove that the $C_{\left(-1-\alpha, \Gamma_{w}^{+}\right)}^{2, \alpha}\left(\mathcal{N}_{f}^{+}\right)$solution of (3.40), (3.41) is of the form $\Psi(r, \theta) \mathbf{e}_{\varphi}$. This statement is proved using the argument as in the proof of Proposition 3.3 in [4] (Method II). Although the arguments to prove this statement are almost the same with those in the proof of Proposition 3.3 in [4] (Method II), since (3.40), (3.41) is different from the problem in Proposition 3.3 in [4] and similar arguments will be used later in the proof of Lemma 18, we present the detailed proof.

Lemma 14. Under the assumption as in Lemma 7 , the $C_{\left(-1-\alpha, \Gamma_{w}^{+}\right)}^{2, \alpha}\left(\mathcal{N}_{f}^{+}\right)$solution of (3.40), (3.41) is of the form $\Psi(r, \theta) \mathbf{e}_{\varphi}$. 
Proof. Let $\boldsymbol{U}=U_{r} \mathbf{e}_{r}+U_{\theta} \mathbf{e}_{\theta}+U_{\varphi} \mathbf{e}_{\varphi}$ be the $C_{\left(-1-\alpha, \Gamma_{w}^{+}\right)}^{2, \alpha}\left(\mathcal{N}_{f}^{+}\right)$solution of (3.40), (3.41). Then, $\left(U_{r}, U_{\theta}, U_{\varphi}\right)$ is in $\left(C_{\left(-1-\alpha,\left\{\theta=\theta_{1}\right\}\right)}^{2, \alpha}\left(\mathcal{N}_{f}^{+, *}\right)\right)^{3}$ satisfying

$$
\begin{aligned}
& \left\|U_{r}\right\|_{2, \alpha, \mathcal{N}_{f}^{+, *}}^{\left(-1-\alpha,\left\{\theta=\theta_{1}\right\}\right)},\left\|U_{\theta}\right\|_{2, \alpha, \mathcal{N}_{f}^{+, *}}^{\left(-1-\alpha,\left\{\theta=\theta_{1}\right\}\right)},\left\|U_{\varphi}\right\|_{2, \alpha, \mathcal{N}_{f}^{+, *}}^{\left(-1-\alpha,\left\{\theta=\theta_{1}\right\}\right)} \\
& \leq C\|\boldsymbol{U}\|_{2, \alpha, \mathcal{N}_{f}^{+}}^{\left(-1-\alpha, \Gamma_{w}^{+}\right)} \leq C C^{*}
\end{aligned}
$$

where $C$ and $C^{*}$ are positive constants depending on $\mathcal{N}_{f}^{+}$and $\alpha$ and given in (3.39), respectively, and satisfies the following spherical coordinate representation of (3.40), (3.41)

$$
\begin{aligned}
& \left\{\begin{array}{c}
\left(\frac{c_{0}^{+2}}{\rho_{0}^{+}\left(c_{0}^{+2}-u_{0}^{+2}\right)}\left(\Delta U_{r}-\frac{2 U_{r}}{r^{2}}-\frac{2}{r^{2} \sin \theta} \partial_{\theta}\left(U_{\theta} \sin \theta\right)-\frac{2}{r^{2} \sin \theta} \partial_{\varphi} U_{\varphi}\right)\right. \\
\left.-\frac{u_{0}^{+2}}{\rho_{0}^{+}\left(c_{0}^{+2}-u_{0}^{+2}\right)} \frac{1}{r^{2}} \partial_{r}\left(r^{2} \partial_{r} U_{r}\right)-\frac{\partial_{r} \rho_{0}^{+}}{\rho_{0}^{+2}} \frac{\partial_{r}\left(r U_{r}\right)}{r}\right)=0 \\
\left(\frac{c_{0}^{+2}}{\rho_{0}^{+}\left(c_{0}^{+2}-u_{0}^{+2}\right)}\left(\Delta U_{\theta}-\frac{U_{\theta}}{r^{2} \sin ^{2} \theta}+\frac{2}{r^{2}} \partial_{\theta} U_{r}-\frac{2 \cos \theta}{r^{2} \sin ^{2} \theta} \partial_{\varphi} U_{\varphi}\right)\right. \\
\left.-\frac{u_{0}^{+2}}{\rho_{0}^{+}\left(c_{0}^{+2}-u_{0}^{+2}\right)} \frac{1}{r^{2}} \partial_{r}\left(r^{2} \partial_{r} U_{\theta}\right)-\frac{\partial_{r} \rho_{0}^{+}}{\rho_{0}^{+2}} \frac{\partial_{r}\left(r U_{\theta}\right)}{r}\right)=0, \\
\left(\frac{c_{0}^{+2}}{\rho_{0}^{+}\left(c_{0}^{+2}-u_{0}^{+2}\right)}\left(\Delta U_{\varphi}-\frac{U_{\varphi}}{r^{2} \sin ^{2} \theta}+\frac{2}{r^{2} \sin \theta} \partial_{\varphi} U_{r}+\frac{2 \cos \theta}{r^{2} \sin { }^{2} \theta} \partial_{\varphi} U_{\theta}\right)\right. \\
\left.-\frac{u_{0}^{+2}}{\rho_{0}^{+}\left(c_{0}^{+2}-u_{0}^{+2}\right)} \frac{1}{r^{2}} \partial_{r}\left(r^{2} \partial_{r} U_{\varphi}\right)-\frac{\partial_{r} \rho_{0}^{+}}{\rho_{0}^{+2}} \partial_{r}\left(r U_{\varphi}\right)\right)=-\mathcal{F}(r, \theta) \\
\text { in } \mathcal{N}_{f}^{+, *}, \quad \text { on } \Gamma_{f}^{*}, \Gamma_{w}^{+, *}, \Gamma_{e x}^{*},
\end{array}\right. \\
& \left(U_{r}, U_{\theta}, U_{\varphi}\right)=(0,0,0)
\end{aligned}
$$

where $\mathcal{F}=\boldsymbol{F}^{\sharp} \cdot \mathbf{e}_{\varphi}$ and $\Delta U_{k}=\frac{1}{r^{2}} \partial_{r}\left(r^{2} \partial_{r} U_{k}\right)+\frac{1}{r^{2} \sin \theta} \partial_{\theta}\left(\sin \theta \partial_{\theta} U_{k}\right)+\frac{1}{r^{2} \sin ^{2} \theta}$ $\partial_{\varphi}^{2} U_{k}$ for $k=r, \theta, \varphi$.

Define

$$
U_{k}^{n}:=\frac{1}{2^{n}} \sum_{k=0}^{2^{n}-1} U_{k}\left(r, \theta, \varphi+\frac{2 \pi k}{2^{n}}\right)
$$

for $k=r, \theta, \varphi$. Then by the definition of $U_{k}^{n}$ for $k=r, \theta, \varphi$ and (3.72),

$$
\left\|U_{r}^{n}\right\|_{2, \alpha, \mathcal{N}_{f}^{+, *}}^{\left(-1-\alpha,\left\{\theta=\theta_{1}\right\}\right)},\left\|U_{\theta}^{n}\right\|_{2, \alpha, \mathcal{N}_{f}^{+, *}}^{\left(-1-\alpha,\left\{\theta=\theta_{1}\right\}\right)},\left\|U_{\varphi}^{n}\right\|_{2, \alpha, \mathcal{N}_{f}^{+, *}}^{\left(-1-\alpha,\left\{\theta=\theta_{1}\right\}\right)} \leq C C^{*} .
$$

By (3.75) and $C_{\left(-1-\alpha,\left\{\theta=\theta_{1}\right\}\right)}^{2, \alpha}\left(\mathcal{N}_{f}^{+, *}\right) \Subset C_{\left(-1-\frac{\alpha}{2},\left\{\theta=\theta_{1}\right\}\right)}^{2, \alpha}\left(\mathcal{N}_{f}^{+, *}\right)$, there exists a subsequence $\left(U_{r}^{n_{k}}, U_{\theta}^{n_{k}}, U_{\varphi}^{n_{k}}\right)$ of $\left(U_{r}^{n}, U_{\theta}^{n}, U_{\varphi}^{n}\right)$ such that $\left(U_{r}^{n_{k}}, U_{\theta}^{n_{k}}, U_{\varphi}^{n_{k}}\right)$ converges in $C_{\left(-1-\frac{\alpha}{2},\left\{\theta=\theta_{1}\right\}\right)}^{2, \alpha}\left(\mathcal{N}_{f}^{+, *}\right)$ as $n_{k} \rightarrow \infty$. Denote its limit by $\left(U_{r}^{*}, U_{\theta}^{*}, U_{\varphi}^{*}\right)$. Then $\left(U_{r}^{*}, U_{\theta}^{*}, U_{\varphi}^{*}\right)$ is independent of $\varphi$ and $\left(U_{r}^{*}, U_{\theta}^{*}, U_{\varphi}^{*}\right) \in\left(C_{\left(-1-\alpha,\left\{\theta=\theta_{1}\right\}\right)}^{2, \alpha}\left(\mathcal{N}_{f}^{+, *}\right)\right)^{3}$.

Since the coefficients of (3.73), $\mathcal{F}$ and the boundary conditions in (3.74) are independent of $\varphi,\left(U_{r}^{n}, U_{\theta}^{n}, U_{\varphi}^{n}\right)$ satisfies (3.73), (3.74) for all $n \in \mathbb{N} \cup\{0\}$. By this fact and the definition of $\left(U_{r}^{*}, U_{\theta}^{*}, U_{\varphi}^{*}\right),\left(U_{r}^{*}, U_{\theta}^{*}, U_{\varphi}^{*}\right)$ satisfies (3.73), (3.74). 
Since $\left(U_{r}^{*}, U_{\theta}^{*}, U_{\varphi}^{*}\right)$ is independent of $\varphi,(3.73)$ satisfied by $\left(U_{r}^{*}, U_{\theta}^{*}, U_{\varphi}^{*}\right)$ is given as

$$
\left\{\begin{array}{c}
\left(\frac { c _ { 0 } ^ { + 2 } } { \rho _ { 0 } ^ { + } ( c _ { 0 } ^ { + 2 } - u _ { 0 } ^ { + 2 } ) } \left(\frac{1}{r^{2}} \partial_{r}\left(r^{2} \partial_{r} U_{r}^{*}\right)+\frac{1}{r^{2} \sin \theta} \partial_{\theta}\left(\sin \theta \partial_{\theta} U_{r}^{*}\right)-\frac{2 U_{r}^{*}}{r^{2}}\right.\right. \\
\left.\left.-\frac{2}{r^{2} \sin \theta} \partial_{\theta}\left(U_{\theta}^{*} \sin \theta\right)\right)-\frac{u_{0}^{+2}}{\rho_{0}^{+}\left(c_{0}^{+2}-u_{0}^{+2}\right)} \frac{1}{r^{2}} \partial_{r}\left(r^{2} \partial_{r} U_{r}^{*}\right)-\frac{\partial_{r} \rho_{0}^{+}}{\rho_{0}^{+2}} \frac{\partial_{r}\left(r U_{r}^{*}\right)}{r}\right)=0 \\
\left(\frac{c_{0}^{+2}}{\rho_{0}^{+}\left(c_{0}^{+2}-u_{0}^{+2}\right)}\left(\frac{1}{r^{2}} \partial_{r}\left(r^{2} \partial_{r} U_{\theta}^{*}\right)+\frac{1}{r^{2} \sin \theta} \partial_{\theta}\left(\sin \theta \partial_{\theta} U_{\theta}^{*}\right)-\frac{U_{\theta}^{*}}{r^{2} \sin ^{2} \theta}+\frac{2}{r^{2}} \partial_{\theta} U_{r}^{*}\right)\right. \\
\left.-\frac{u_{0}^{+2}}{\rho_{0}^{+}\left(c_{0}^{+2}-u_{0}^{+2}\right)} \frac{1}{r^{2}} \partial_{r}\left(r^{2} \partial_{r} U_{\theta}^{*}\right)-\frac{\partial_{r} \rho_{0}^{+}}{\rho_{0}^{+2}} \frac{\partial_{r}\left(r U_{\theta}^{*}\right)}{r}\right)=0, \\
\left(\frac{c_{0}^{+2}}{\rho_{0}^{+}\left(c_{0}^{+2}-u_{0}^{+2}\right)}\left(\frac{1}{r^{2}} \partial_{r}\left(r^{2} \partial_{r} U_{\varphi}^{*}\right)+\frac{1}{r^{2} \sin \theta} \partial_{\theta}\left(\sin \theta \partial_{\theta} U_{\varphi}^{*}\right)-\frac{U_{\varphi}^{*}}{r^{2} \sin ^{2} \theta}\right)\right. \\
\left.-\frac{u_{0}^{+2}}{\rho_{0}^{+}\left(c_{0}^{+2}-u_{0}^{+2}\right)} \frac{1}{r^{2}} \partial_{r}\left(r^{2} \partial_{r} U_{\varphi}^{*}\right)-\frac{\partial_{r} \rho_{0}^{+}}{\rho_{0}^{+2}} \partial_{r}\left(r U_{\varphi}^{*}\right)\right)=-\mathcal{F}(r, \theta)
\end{array}\right.
$$

in $\mathcal{N}_{f}^{+, *}$.

Note that the first and second equation of (3.76) is not coupled with the third equation of (3.76).

Let $\Psi=U_{\varphi}^{*}$. Using the third equation of (3.76) and the facts that $U_{\varphi}^{*} \in$ $C_{\left(-1-\alpha,\left\{\theta=\theta_{1}\right\}\right)}^{2, \alpha}\left(\mathcal{N}_{f}^{+, *}\right)$ and $\boldsymbol{F}^{\sharp}$ is an axisymmetric function in $C_{\left(1-\alpha, \Gamma_{w}^{+}\right)}^{\alpha}\left(\mathcal{N}_{f}^{+}\right)$, it can be checked that $\Psi=\partial_{\theta \theta} \Psi=0$ on $\theta=0$. By this fact and $U_{\varphi}^{*} \in$ $C_{\left(-1-\alpha,\left\{\theta=\theta_{1}\right\}\right)}^{2, \alpha}\left(\mathcal{N}_{f}^{+, *}\right)$, we have that $\Psi \mathbf{e}_{\varphi} \in C_{\left(-1-\alpha, \Gamma_{w}^{+}\right)}^{2, \alpha}\left(\mathcal{N}_{f}^{+}\right)$. From (3.76) and (3.74), one can see that $\left(0,0, U_{\varphi}^{*}\right)$ is a solution of (3.73), (3.74). Combining this fact with the fact that $\Psi \mathbf{e}_{\varphi} \in C_{\left(-1-\alpha, \Gamma_{w}^{+}\right)}^{2, \alpha}\left(\mathcal{N}_{f}^{+}\right)$, we have that $\Psi \mathbf{e}_{\varphi}$ is a $C_{\left(-1-\alpha, \Gamma_{w}^{+}\right)}^{2, \alpha}\left(\mathcal{N}_{f}^{+}\right)$ solution of (3.40), (3.41). By Lemma 9, a $C_{\left(-1-\alpha, \Gamma_{w}^{+}\right)}^{2, \alpha}\left(\mathcal{N}_{f}^{+}\right)$solution of (3.40), (3.41) is unique. Therefore, $\boldsymbol{U}=\Psi \mathbf{e}_{\varphi}$. This finishes the proof.

\subsection{Initial value problem of a transport equation with an axisymmetric divergence-free coefficient}

The initial value problems of a transport equation in $\left(\mathrm{B}^{\prime}\right)$ are of the form

$$
\begin{aligned}
& \nabla \times\left(\left(\Phi_{0}^{+}+\Psi\right) \mathbf{e}_{\varphi}\right) \cdot \nabla Q=0 \text { in } \mathcal{N}_{f}^{+}, \\
& Q=Q_{e n} \text { on } \Gamma_{f},
\end{aligned}
$$

where $\Psi \mathbf{e}_{\varphi}: \mathcal{N}_{f}^{+} \rightarrow \mathbb{R}^{3}$ and $Q_{\text {en }}: \Gamma_{f} \rightarrow \mathbb{R}$ are axisymmetric functions. Here, (3.77) is a transport equation whose coefficient is an axisymmetric and divergencefree vector field. Thus, the stream function of the coefficient vector field of (3.77) can be defined as in (2.23). We will find a solution of (3.77), (3.78) and obtain the regularity and uniqueness of solutions of (3.77), (3.78) using the stream function of the coefficient vector field of (3.77) and the solution expression given by using the stream function. This will be done in the proof of the following lemma: 
Lemma 15. Suppose that $f$ is as in Lemma 4. Let $\delta_{6}$ be a positive constant such that for such $f$, if $\left\|\nabla \times\left(\Psi \mathbf{e}_{\varphi}\right)\right\|_{0,0, \mathcal{N}_{f}^{+}} \leq \delta_{6}$, then

$$
\begin{aligned}
& \nabla \times\left(\left(\Phi_{0}^{+}+\Psi\right) \mathbf{e}_{\varphi}\right) \cdot \mathbf{e}_{r}>c^{*} \text { in } \overline{\mathcal{N}_{f}^{+}} \\
& \quad \text { and } \nabla \times\left(\left(\Phi_{0}^{+}+\Psi\right) \mathbf{e}_{\varphi}\right) \cdot \boldsymbol{v}_{f}>c^{*} \text { on } \overline{\Gamma_{f}}
\end{aligned}
$$

where $c^{*}$ is a positive constant depending on $\rho_{0}^{+}, u_{0}^{+}, r_{s}, r_{1}, \delta_{1}$ and $\delta_{6}$, and $\boldsymbol{v}_{f}$ is the unit normal vector on $\Gamma_{f}$ pointing toward $\mathcal{N}_{f}^{+}$. Suppose that $\Psi \mathbf{e}_{\varphi}: \mathcal{N}_{f}^{+} \rightarrow \mathbb{R}^{3}$ is an axisymmetric function in $C^{1, \alpha}\left(\overline{\mathcal{N}_{f}^{+}}\right)$satisfying

$$
\Psi=\frac{f\left(\theta_{1}\right) \Psi\left(f\left(\theta_{1}\right), \theta_{1}\right)}{r} \text { on } \overline{\Gamma_{w}^{+}}
$$

and

$$
\left\|\nabla \times\left(\Psi \mathbf{e}_{\varphi}\right)\right\|_{0, \alpha, \mathcal{N}_{f}^{+}} \leq \delta_{6}
$$

Suppose finally that $Q_{e n}: \Gamma_{f} \rightarrow \mathbb{R}$ is an axisymmetric function in $C_{\left(-\alpha, \partial \Gamma_{f}\right)}^{1, \alpha}\left(\Gamma_{f}\right)$. Then the problem (3.77), (3.78) has a unique axisymmetric $C^{0}\left(\overline{\mathcal{N}_{f}^{+}}\right) \cap C^{1}\left(\mathcal{N}_{f}^{+}\right)$ solution

$$
Q=Q_{\text {en }}(\mathcal{L}) \text { in } \mathcal{N}_{f}^{+},
$$

where $\mathcal{L}=k^{-1} \circ V$ with $V=2 \pi r \sin \theta\left(\Phi_{0}^{+}+\Psi\right)$ and $k(\theta)=V(f(\theta), \theta)$. Furthermore, this solution $Q$ satisfies

$$
\|Q\|_{1, \alpha, \mathcal{N}_{f}^{+}}^{\left(-\alpha, \Gamma_{+}^{+}\right)} \leq C\left\|Q_{e n}\right\|_{1, \alpha, \Gamma_{f}}^{\left(-\alpha, \partial \Gamma_{f}\right)}
$$

where $C$ is a positive constant depending on $\rho_{0}^{+}, u_{0}^{+}, r_{s}, r_{1}, \theta_{1}, \alpha, \delta_{1}$ and $\delta_{6}$.

Remark 8. The existence of $\delta_{6}$ is obtained from the facts that $\nabla \times\left(\Phi_{0}^{+} \mathbf{e}_{\varphi}\right) \cdot \mathbf{e}_{r}=$ $\rho_{0}^{+} u_{0}^{+}>0$ in $\overline{\mathcal{N}_{r_{s}-\delta_{1}}^{+}}$and $\boldsymbol{v}_{f} \cdot \mathbf{e}_{r}>0$ on $\overline{\Gamma_{f}}$ for $f$ given in Lemma 4.

Remark 9. If $\Psi \mathbf{e}_{\varphi}$ satisfies (3.79), then $V=2 \pi r \sin \theta\left(\Phi_{0}^{+}+\Psi\right)$ satisfies

$$
\partial_{\theta} V>0 \text { in } \mathcal{N}_{f}^{+} \backslash\{\theta=0\} \text { and } \partial_{\theta}(V(f(\theta), \theta))>0 \text { for } \theta \in\left(0, \theta_{1}\right) .
$$

This condition will be used to construct the stream surfaces of $\nabla \times\left(\left(\Phi_{0}^{+}+\Psi\right) \mathbf{e}_{\varphi}\right)$ in $\mathcal{N}_{f}^{+}$in the proof of Lemma 15.

Proof. 1. Construct the stream surfaces of $\nabla \times\left(\left(\Phi_{0}^{+}+\Psi\right) \mathbf{e}_{\varphi}\right)$ in $\overline{\mathcal{N}_{f}^{+}}$.

Let us define $V:=2 \pi r \sin \theta\left(\Phi_{0}^{+}+\Psi\right)$. By the fact that $\Psi \mathbf{e}_{\varphi}$ and $\Phi_{0}^{+} \mathbf{e}_{\varphi}$ are axisymmetric functions in $C^{1, \alpha}\left(\overline{\mathcal{N}_{f}^{+}}\right), V$ is an axisymmetric function in $C^{1, \alpha}\left(\overline{\mathcal{N}_{f}^{+}}\right)$ (see Lemma 1). By (3.79), $\partial_{\theta} V>0$ in $\mathcal{N}_{f}^{+} \backslash\{x=y=0\}$. Using these facts, we apply the implicit function theorem to $V$. Then we obtain that for any $\left(r^{\sharp}, \theta^{\sharp}, \varphi^{\sharp}\right) \in$ $\mathcal{N}_{f}^{+, *}$, there exists a unique $C^{1}$ surface $\theta=h_{\left(r^{\sharp}, \theta^{\sharp}, \varphi^{\sharp}\right)}(r, \varphi)$ defined near $\left(r^{\sharp}, \varphi^{\sharp}\right)$ 
such that $\theta^{\sharp}=h_{\left(r^{\sharp}, \theta^{\sharp}, \varphi^{\sharp}\right)}\left(r^{\sharp}, \varphi^{\sharp}\right)$ and $V\left(r, h_{\left(r^{\sharp}, \theta^{\sharp}, \varphi^{\sharp}\right)}(r, \varphi), \varphi\right)=V\left(r^{\sharp}, \theta^{\sharp}, \varphi^{\sharp}\right)$. Since $V$ is axisymmetric, this surface is axisymmetric. We denote $\theta=h_{\left(r^{\sharp}, \theta^{\sharp}, \varphi^{\sharp}\right)}(r, \varphi)$ by $\theta=h_{\left(r^{\sharp}, \theta^{\sharp}\right)}(r)$.

By $V_{0}^{+}=V_{0}^{+}\left(f\left(\theta_{1}\right), \theta_{1}\right)$ on $\overline{\Gamma_{w}^{+}}$and (3.80), $V=V\left(f\left(\theta_{1}\right), \theta_{1}\right)$ on $\overline{\Gamma_{w}^{+}}$. By this fact, $V=0$ on $\overline{\mathcal{N}_{f}^{+}} \cap\{x=y=0\}$ and $\partial_{\theta} V>0$ in $\mathcal{N}_{f}^{+} \backslash\{x=y=0\}, \theta=$ $h_{\left(r^{\sharp}, \theta^{\sharp}\right)}(r)$ is defined until it reaches $\Gamma_{f}$ or $\Gamma_{e x}$ not touching $\overline{\mathcal{N}_{f}^{+}} \cap\{x=y=0\}$ or $\overline{\Gamma_{w}^{+}}$. Note that by the facts that $\partial_{\theta}(V(f(\theta), \theta))>0$ for $\theta \in\left(0, \theta_{1}\right)$ and $\partial_{\theta} V\left(r_{1}, \theta\right)>$ 0 for $\theta \in\left(0, \theta_{1}\right)$ obtained from (3.79), the surface $\theta=h_{\left(r^{\sharp}, \theta^{\sharp}\right)}(r)$ intersects with $\Gamma_{f}$ and $\Gamma_{e x}$ once, respectively. Collect $\theta=h_{\left(r^{\sharp}, \theta^{\sharp}\right)}(r)$ for all $\left(r^{\sharp}, \theta^{\sharp}\right) \in\left(f\left(\theta^{\sharp}\right), r_{1}\right) \times$ $\left(0, \theta_{1}\right), \overline{\mathcal{N}_{f}^{+}} \cap\{x=y=0\}$ and $\overline{\Gamma_{w}^{+}}$. Then we have the entire level surfaces of $V$ in $\overline{\mathcal{N}_{f}^{+}}$. By $\partial_{\theta} V>0$ in $\mathcal{N}_{f}^{+} \backslash\{x=y=0\}$, the values of $V$ on distinct level surfaces of $V$ in $\overline{\mathcal{N}_{f}^{+}}$are different from each other.

2. Find a solution of (3.77), (3.78).

By (2.27), (3.77) can be written as

$$
\nabla^{\perp} V \cdot \nabla Q=0
$$

Using this form of (3.77), it can be checked that 1) if $Q$ is in $C^{1}\left(\mathcal{N}_{f}^{+}\right)$and $Q=$ constant on any curve on any level surface of $V$ in $\mathcal{N}_{f}^{+}$whose $\varphi$ argument is fixed (in the case when a level surface of $V$ is $\mathcal{N}_{f}^{+} \cap\{x=y=0\}, Q=$ constant on $\mathcal{N}_{f}^{+} \cap\{x=y=0\}$ ), then $Q$ is a solution of (3.77) and that 2) if $Q$ is a $C^{1}$ solution of (3.77), then $Q=$ constant on any curve on any level surface of $V$ in $\mathcal{N}_{f}^{+}$whose $\varphi$ argument is fixed. Denote the $\theta$-argument of the intersection points of $\Gamma_{f}$ and the level surface of $V$ in $\overline{\mathcal{N}_{f}^{+}}$passing through $\mathrm{x} \in \overline{\mathcal{N}_{f}^{+}}$by $\mathcal{L}(\mathrm{x})$. Since each level surface of $V$ in $\overline{\mathcal{N}_{f}^{+}}$intersects with $\overline{\Gamma_{f}}$ where the value of $V$ on $\overline{\Gamma_{f}}$ is equal to the value of $V$ on the level surface, $\mathcal{L}(\mathrm{x})$ is given by

$$
\mathcal{L}(\mathrm{x}):=k^{-1} \circ V(\mathrm{x}),
$$

where $k(\theta)=V(f(\theta), \theta)$.

By 2), a $C^{0}\left(\overline{\mathcal{N}_{f}^{+}}\right) \cap C^{1}\left(\mathcal{N}_{f}^{+}\right)$solution of (3.77), (3.78) has the form

$$
Q(r, \theta, \varphi)= \begin{cases}Q_{e n}(\mathcal{L}(r, \theta), \varphi) & \text { if }(r, \theta) \in\left[f(\theta), r_{1}\right] \times\left(0, \theta_{1}\right] \\ Q_{e n}(\mathcal{L}(r, 0), 0) & \text { if }(r, \theta) \in\left[f(0), r_{1}\right] \times\{0\}\end{cases}
$$

Since $Q_{e n}$ is axisymmetric, this can be written as

$$
Q=Q_{e n}(\mathcal{L})
$$

Let $Q=Q_{\text {en }}(\mathcal{L})$. One can see that $Q$ is a constant on any level surface of $V$ in $\overline{\mathcal{N}_{f}^{+}}$and satisfies (3.78). Thus, by 1), if $Q \in C^{0}\left(\overline{\mathcal{N}_{f}^{+}}\right) \cap C^{1}\left(\mathcal{N}_{f}^{+}\right)$, then $Q$ is a $C^{0}\left(\overline{\mathcal{N}_{f}^{+}}\right) \cap C^{1}\left(\mathcal{N}_{f}^{+}\right)$solution of (3.77), (3.78). 
3. Estimate $\|Q\|_{1, \alpha, \mathcal{N}_{f}^{+}}^{\left(-\alpha, \Gamma_{w}^{+}\right)}$.

Since $\mathcal{L} \in C^{0}\left(\overline{\mathcal{N}_{f}^{+}}\right)$and $Q_{e n} \in C^{0}\left(\overline{\Gamma_{f}}\right), Q$ is in $C^{0}\left(\overline{\mathcal{N}_{f}^{+}}\right)$. It directly follows from the definition of $Q$

$$
\|Q\|_{0,0, \mathcal{N}_{f}^{+}}=\left\|Q_{e n}\right\|_{0,0, \Gamma_{f}} .
$$

Thus, to estimate $\|Q\|_{1, \alpha, \mathcal{N}_{f}^{+}}^{\left(-\alpha, \Gamma^{+}\right)}$, it is enough to estimate $\|D Q\|_{\alpha, \mathcal{N}_{f}^{+}}^{\left(1-\alpha, \Gamma_{w}^{+}\right)}$. We estimate $\|D Q\|_{\alpha, \mathcal{N}_{f}^{+}}^{\left(1-\alpha, \Gamma_{w}^{+}\right)}$.

By direct computation,

$$
\begin{aligned}
D Q & =Q_{e n}^{\prime}(\mathcal{L}) D \mathcal{L} \\
& =Q_{e n}^{\prime}(\mathcal{L}) \frac{D V}{\left.\left(\partial_{r} V(f(\vartheta), \vartheta) f^{\prime}(\vartheta)+\partial_{\vartheta} V(f(\vartheta), \vartheta)\right)\right|_{\vartheta=\mathcal{L}}} .
\end{aligned}
$$

To estimate $\|D Q\|_{\alpha, \mathcal{N}_{f}^{+}}^{\left(1-\alpha, \Gamma_{w}^{+}\right)}$, we estimate $\|D \mathcal{L}\|_{0, \alpha, \mathcal{N}_{f}^{+}}$.

Write $D \mathcal{L}$ as

$$
\frac{\frac{D V}{2 \pi \sin \theta}}{\left.\left(\nabla^{\perp} V\right)(f(\vartheta), \vartheta) \cdot \boldsymbol{v}_{f}(\vartheta) f^{2}(\vartheta) \sqrt{1+\left(\frac{f^{\prime}(\vartheta)}{f(\vartheta)}\right)^{2}}\right|_{\vartheta=\mathcal{L}}} \cdot \frac{\sin \theta}{\sin \mathcal{L}}
$$

Here, we used the definition of $\nabla^{\perp} V$ and the spherical coordinate expression of $\boldsymbol{v}_{f}$. Using (3.3) and (3.79), it is easily seen that

$$
\sup _{\mathcal{N}_{f}^{+}}\left|\left(\nabla^{\perp} V\right)(f(\vartheta), \vartheta) \cdot \boldsymbol{v}_{f}(\vartheta) f^{2}(\vartheta) \sqrt{1+\left(\frac{f^{\prime}(\vartheta)}{f(\vartheta)}\right)^{2}}\right|_{\vartheta=\mathcal{L}} \mid>c^{* *},
$$

where $c^{* *}$ is a positive constant depending on $c^{*}, r_{s}$ and $\delta_{1}$. Using (3.3) and (3.81), it is also easily seen that

$$
\left\|\frac{D V}{2 \pi \sin \theta}\right\|_{0, \alpha, \mathcal{N}_{f}^{+}} \leq C
$$

and

$$
\begin{aligned}
& \left\|\left.\left(\nabla^{\perp} V\right)(f(\vartheta), \vartheta) \cdot v_{f}(\vartheta) f^{2}(\vartheta) \sqrt{1+\left(\frac{f^{\prime}(\vartheta)}{f(\vartheta)}\right)^{2}}\right|_{\vartheta=\mathcal{L}}\right\| \|_{0, \alpha, \mathcal{N}_{f}^{+}} \\
& \quad \leq C\|D \mathcal{L}\|_{0,0, \mathcal{N}_{f}^{+}}
\end{aligned}
$$

where $C s$ are positive constants depending on $\rho_{0}^{+}, u_{0}^{+}, r_{s}, \alpha, \delta_{1}$ and $\delta_{6}$. By these three estimates, one can see that to estimate $\|D \mathcal{L}\|_{0, \alpha, \mathcal{N}_{f}^{+}}$, it is enough to estimate $\left\|\frac{\theta}{\mathcal{L}}\right\|_{0, \alpha, \mathcal{N}_{f}^{+}}$. To estimate $\|D \mathcal{L}\|_{0, \alpha, \mathcal{N}_{f}^{+}}$, we prove the following claim: 
Claim. There exists a positive constant $C$ depending on $\rho_{0}^{+}, u_{0}^{+}, r_{s}, r_{1}, \theta_{1}, \alpha$, $\delta_{1}$ and $\delta_{6}$ such that

$$
\left\|\frac{\theta}{\mathcal{L}}\right\|_{0, \alpha, \mathcal{N}_{f}^{+}} \leq C
$$

Proof of Claim. To simplify our argument, we assume that $f=r_{s}$.

First, we estimate $\left\|\frac{\theta}{\mathcal{L}}\right\|_{0,0, \mathcal{N}_{r_{s}}^{+}}$. By the definition of $\mathcal{L}$,

$$
V\left(r_{s}, \mathcal{L}(r, \theta)\right)=V(r, \theta)
$$

for all $(r, \theta) \in\left[r_{s}, r_{1}\right] \times\left[0, \theta_{1}\right]$. Write this as

$$
\begin{aligned}
& \int_{0}^{\theta}\left(\rho_{0}^{+} u_{0}^{+}+\nabla \times\left(\Psi \mathbf{e}_{\varphi}\right) \cdot \mathbf{e}_{r}\right)(r, \xi) r^{2} \sin \xi \mathrm{d} \xi \\
& \quad=\int_{0}^{\mathcal{L}(r, \theta)}\left(\rho_{0}^{+} u_{0}^{+}+\nabla \times\left(\Psi \mathbf{e}_{\varphi}\right) \cdot \mathbf{e}_{r}\right)\left(r_{s}, \xi\right) r_{s}^{2} \sin \xi \mathrm{d} \xi .
\end{aligned}
$$

By (3.79), (3.81) and the first equation of (2.14), we have from this equation

$$
\int_{0}^{\theta} c^{*} r^{2} \sin \xi \mathrm{d} \xi \leq \int_{0}^{\mathcal{L}(r, \theta)}\left(m_{0}+\delta_{6} r_{s}^{2}\right) \sin \xi \mathrm{d} \xi .
$$

Using $\frac{\sin \theta_{1}}{\theta_{1}} \xi \leq \sin \xi \leq \xi$ for $\xi \in\left[0, \theta_{1}\right]$, change $\sin \xi$ in the integrands in the left and right hand side of the above inequalities to $\frac{\sin \theta_{1}}{\theta_{1}} \xi$ and $\xi$, respectively, and then integrate the resultant terms. Then we obtain

$$
\frac{\sin \theta_{1}}{\theta_{1}} c^{*} r^{2} \frac{\theta^{2}}{2} \leq\left(m_{0}+\delta_{6} r_{s}^{2}\right) \frac{\mathcal{L}^{2}(r, \theta)}{2} .
$$

From this inequality, we have

$$
\sqrt{\frac{\sin \theta_{1}}{\theta_{1}} \frac{c^{*} r_{s}^{2}}{m_{0}+\delta_{6} r_{s}^{2}}} \theta \leq \mathcal{L}(r, \theta) .
$$

This holds for all $(r, \theta) \in\left[r_{s}, r_{1}\right] \times\left[0, \theta_{1}\right]$. Hence,

$$
\left\|\frac{\theta}{\mathcal{L}}\right\|_{0,0, \mathcal{N}_{r_{s}}^{+}} \leq C
$$

where $C$ is a positive constant depending on $\rho_{0}^{+}, u_{0}^{+}, r_{s}, \theta_{1}, \delta_{6}$ and $c^{*}$.

Next, we estimate $\left[\frac{\theta}{\mathcal{L}}\right]_{0, \alpha, \mathcal{N}_{r_{s}}^{+}}$. By (3.88), we can obtain an estimate of $\left[\frac{\theta}{\mathcal{L}}\right]_{0, \alpha, \mathcal{N}_{r_{s}}^{+}}$ by obtaining

$$
\left|\frac{\theta}{\mathcal{L}(r, \theta)}-\frac{\theta^{\prime}}{\mathcal{L}\left(r^{\prime}, \theta^{\prime}\right)}\right| \leq C \varepsilon^{\alpha}
$$

for all $(r, \theta),\left(r^{\prime}, \theta^{\prime}\right) \in\left[r_{s}, r_{1}\right] \times\left[0, \theta_{1}\right]$ satisfying $\varepsilon \leq \varepsilon_{0}$ for a positive constant $\varepsilon_{0}$ and a positive constant $C$ where $\varepsilon:=\sqrt{\left|r^{\prime}-r\right|^{2}+\left|\theta^{\prime}-\theta\right|^{2}}$. We obtain this estimate. 
Hereafter, to simplify our notation, we denote $\mathcal{L}\left(r^{\prime}, \theta^{\prime}\right), \mathcal{L}(r, \theta)$ and $\Phi_{0}^{+}+\Psi$ by $\mathcal{L}^{\prime}, \mathcal{L}$ and $\Phi$, respectively.

By the definition of $\mathcal{L}$,

$$
V\left(r_{s}, \mathcal{L}^{\prime}\right)-V\left(r_{s}, \mathcal{L}\right)=V\left(r^{\prime}, \theta^{\prime}\right)-V(r, \theta)
$$

Write this as

$$
\begin{aligned}
\int_{\mathcal{L}}^{\mathcal{L}^{\prime}}\left(\nabla \times\left(\Phi \mathbf{e}_{\varphi}\right) \cdot \mathbf{e}_{r}\right)\left(r_{s}, \xi\right) r_{s}^{2} \sin \xi \mathrm{d} \xi \\
=\int_{\theta}^{\theta^{\prime}}\left(\nabla \times\left(\Phi \mathbf{e}_{\varphi}\right) \cdot \mathbf{e}_{r}\right)(r, \xi) r^{2} \sin \xi d \xi \\
\quad+\int_{r}^{r^{\prime}}\left(\nabla \times\left(\Phi \mathbf{e}_{\varphi}\right)\right)\left(t, \theta^{\prime}\right) \cdot \mathbf{e}_{\theta}\left(\theta^{\prime}\right) t \sin \theta^{\prime} d t
\end{aligned}
$$

Using (3.90), we find an upper bound of $\mathcal{L}^{\prime}$. Using (3.81), the fact that $\Phi_{0}^{+} \mathbf{e}_{\varphi} \in$ $C^{\infty}\left(\overline{\mathcal{N}_{r_{s}}^{+}}\right)$and $\frac{\sin \theta_{1}}{\theta_{1}} \xi \leq \sin \xi \leq \xi$ for $\xi \in\left[0, \theta_{1}\right]$, we have, for $\mathcal{L}^{\prime} \geq \mathcal{L}$,

$$
\begin{aligned}
\int_{\mathcal{L}}^{\mathcal{L}^{\prime}} & \left(\left(\nabla \times\left(\Phi \mathbf{e}_{\varphi}\right) \cdot \mathbf{e}_{r}\right)\left(r_{s}, \mathcal{L}\right)-C(\xi-\mathcal{L})^{\alpha}\right) r_{s}^{2} \frac{\sin \theta_{1}}{\theta_{1}} \xi \mathrm{d} \xi \\
\leq & \left(\nabla \times\left(\Phi \mathbf{e}_{\varphi}\right) \cdot \mathbf{e}_{r}\right)(r, \theta) r^{2} \frac{\theta^{\prime 2}-\theta^{2}}{2} \\
\quad+ & C r^{2}\left(\frac{1}{\alpha+1}\left|\theta^{\prime}-\theta\right|^{\alpha+1} \theta^{\prime}-\frac{1}{(\alpha+1)(\alpha+2)}\left|\theta^{\prime}-\theta\right|^{\alpha+2}\right) \\
& +\left(\nabla \times\left(\Phi \mathbf{e}_{\varphi}\right) \cdot \mathbf{e}_{\theta}\right)\left(r, \theta^{\prime}\right) \sin \theta^{\prime} \frac{r^{\prime 2}-r^{2}}{2} \\
& +C \sin \theta^{\prime}\left(\frac{1}{\alpha+1}\left|r^{\prime}-r\right|^{\alpha+1} r^{\prime}-\frac{1}{(\alpha+1)(\alpha+2)}\left|r^{\prime}-r\right|^{\alpha+2}\right)(=:(u)),
\end{aligned}
$$

where the right hand side is an upper bound of the right hand side of (3.90) and $C$ is a positive constant depending on $\rho_{0}^{+}, u_{0}^{+}, \mathcal{N}_{r_{s}}^{+}, \alpha$ and $\delta_{6}$. From this inequality, we get

$$
\begin{aligned}
& \left(\nabla \times\left(\Phi \mathbf{e}_{\varphi}\right) \cdot \mathbf{e}_{r}\right)\left(r_{s}, \mathcal{L}\right) \frac{\sin \theta_{1}}{\theta_{1}} r_{s}^{2} \frac{\mathcal{L}^{\prime 2}-\mathcal{L}^{2}}{2} \\
& -C r_{s}^{2} \frac{\sin \theta_{1}}{\theta_{1}}\left(\frac{1}{\alpha+1}\left(\mathcal{L}^{\prime}-\mathcal{L}\right)^{\alpha+1} \mathcal{L}^{\prime}-\frac{1}{(\alpha+1)(\alpha+2)}\left(\mathcal{L}^{\prime}-\mathcal{L}\right)^{\alpha+2}\right) \leq(u) .
\end{aligned}
$$

Using this inequality, we have that for each $(r, \theta) \in\left[r_{s}, r_{1}\right] \times\left[0, \theta_{1}\right]$, there exists positive constants $\varepsilon_{(r, \theta)}<1$ and $C_{(r, \theta)}$ such that for any $\left(r^{\prime}, \theta^{\prime}\right)$ satisfying $\varepsilon \leq \varepsilon_{(r, \theta)}$,

$$
\mathcal{L}^{\prime} \leq \theta^{\prime}\left(\frac{\mathcal{L}}{\theta}+C_{(r, \theta)} \varepsilon^{\alpha}\right)
$$


Note that when $(r, \theta) \in\left[r_{s}, r_{1}\right] \times\{0\}$, there exists a positive constant $\varepsilon_{(r, \theta)}<1$ such that for any $\left(r^{\prime}, \theta^{\prime}\right)$ satisfying $\varepsilon \leq \varepsilon_{(r, \theta)}$,

$$
\mathcal{L}^{\prime} \leq \theta^{\prime}\left(\sqrt{\frac{r^{2}\left(\nabla \times\left(\Phi \mathbf{e}_{\varphi}\right) \cdot \mathbf{e}_{r}\right)(r, 0)}{r_{s}^{2}\left(\nabla \times\left(\Phi \mathbf{e}_{\varphi}\right) \cdot \mathbf{e}_{r}\right)\left(r_{s}, 0\right)}}+C_{(r, \theta)} \varepsilon^{\alpha}\right) .
$$

Let $\bar{\varepsilon}_{0}=\inf _{(r, \theta) \in\left[r_{s}, r_{1}\right] \times\left[0, \theta_{1}\right]} \varepsilon_{(r, \theta)}$ and $C=\sup _{(r, \theta) \in\left[r_{s}, r_{1}\right] \times\left[0, \theta_{1}\right]} C_{(r, \theta)}$. Then by the above statement,

$$
\frac{\mathcal{L}^{\prime}}{\theta^{\prime}} \leq \frac{\mathcal{L}}{\theta}+C \varepsilon^{\alpha}
$$

for all $(r, \theta),\left(r^{\prime}, \theta^{\prime}\right)$ satisfying $\varepsilon \leq \bar{\varepsilon}_{0}$. This gives

$$
\frac{\theta}{\mathcal{L}}-\frac{\theta^{\prime}}{\mathcal{L}^{\prime}} \leq C \varepsilon^{\alpha}
$$

for all $(r, \theta),\left(r^{\prime}, \theta^{\prime}\right)$ satisfying $\varepsilon \leq \bar{\varepsilon}_{0}$ for a positive constant $C$.

Similarly, we can obtain

$$
-C \varepsilon^{\alpha} \leq \frac{\theta}{\mathcal{L}}-\frac{\theta^{\prime}}{\mathcal{L}^{\prime}}
$$

for all $(r, \theta),\left(r^{\prime}, \theta^{\prime}\right)$ satisfying $\varepsilon \leq \underline{\varepsilon}_{0}$ for a positive constant $C$ and a positive constant $\underline{\varepsilon}_{0}$. Let $\varepsilon_{0}=\min \left(\bar{\varepsilon}_{0}, \underline{\varepsilon}_{0}\right)$. Choose $\varepsilon_{(r, \theta)}$ so that it can depend on $\rho_{0}^{+}, u_{0}^{+}$, $\mathcal{N}_{r_{s}}^{+}, \alpha$ and $\delta_{6}$. Then $\bar{\varepsilon}_{0}$ depends on $\rho_{0}^{+}, u_{0}^{+}, \mathcal{N}_{r_{s}}^{+}, \alpha$ and $\delta_{6}$. In the same way, we have $\underline{\varepsilon}_{0}$ depends on $\rho_{0}^{+}, u_{0}^{+}, \mathcal{N}_{r_{s}}^{+}, \alpha$ and $\delta_{6}$. Thus, $\varepsilon_{0}$ depends on $\rho_{0}^{+}, u_{0}^{+}, \mathcal{N}_{r_{s}}^{+}, \alpha$ and $\delta_{6}$. This finishes the proof of Claim.

By (3.84)-(3.87), we have

$$
\|D \mathcal{L}\|_{0, \alpha, \mathcal{N}_{f}^{+}} \leq C
$$

for a positive constant $C$. It can be easily shown that there exists a positive constant $C$ such that

$$
\left|\theta-\theta_{1}\right| \leq C\left|\mathcal{L}(r, \theta)-\theta_{1}\right|
$$

for all $(r, \theta) \in\left[f(\theta), r_{1}\right] \times\left[0, \theta_{1}\right]$. Using these two estimates, estimate $\|D Q\|$ $\left(1-\alpha, \Gamma_{w}^{+}\right)$
$\alpha, \mathcal{N}_{f}^{+}$

$$
\|D Q\|_{\alpha, \mathcal{N}_{f}^{+}}^{\left(1-\alpha, \Gamma_{w}^{+}\right)} \leq C
$$

where $C$ is a positive constant depending on $\rho_{0}^{+}, u_{0}^{+}, r_{s}, r_{1}, \alpha, \delta_{1}$ and $\delta_{6}$.

4. By the result in Step 3, $Q$ is a $C^{0}\left(\overline{\mathcal{N}_{f}^{+}}\right) \cap C^{1}\left(\mathcal{N}_{f}^{+}\right)$solution of (3.77), (3.78). By 2), a $C^{0}\left(\overline{\mathcal{N}_{f}^{+}}\right) \cap C^{1}\left(\mathcal{N}_{f}^{+}\right)$solution of (3.77), (3.78) is unique. This finishes the proof. 


\subsection{Proof of Proposition 2.}

Using the results in $\S 3.2$ and $\S 3.3$, we prove Proposition 2.

Proof of Proposition 2 (Existence) Suppose that $\left(\rho_{-}, \mathbf{u}_{-}, p_{-}, p_{e x}, f_{s}^{\prime}\right)$ is as in Problem 3 for $\alpha \in\left(\frac{2}{3}, 1\right)$ and $\sigma \in\left(0, \bar{\sigma}_{3}\right]$ where $\bar{\sigma}_{3}$ is a positive constant to be determined later.

For the same $\alpha$ and $\sigma$ and $M_{1}>0$ to be determined later, define

$$
\begin{aligned}
\mathcal{P}\left(M_{1}\right)= & \left\{\left(f(0), \Psi \mathbf{e}_{\varphi}\right) \in \mathbb{R} \times C_{\left(-1-\alpha, \Gamma_{w, r_{s}+f_{s}}^{+}\right)}^{2, \alpha}\left(\mathcal{N}_{r_{s}+f_{s}}^{+}\right) \mid\right. \\
& \Psi \mathbf{e}_{\varphi}=\frac{f\left(\theta_{1}\right) \Psi\left(f\left(\theta_{1}\right), \theta_{1}\right)}{r} \mathbf{e}_{\varphi} \text { on } \Gamma_{w, r_{s}+f_{s}}^{+}, \\
& \left.\left|f(0)-r_{s}\right|+\left\|\Psi \mathbf{e}_{\varphi}\right\|_{2, \alpha, \mathcal{N}_{r_{s}+f_{s}}^{+}}^{\left(-1-\alpha, \Gamma_{w, r_{s}+f_{s}}^{+}\right)} \leq M_{1} \sigma\right\} .
\end{aligned}
$$

By the definition of $\mathcal{P}\left(M_{1}\right), \mathcal{P}\left(M_{1}\right)$ is a compact convex subset of $\mathbb{R} \times C_{\left(-1-\frac{\alpha}{2}, \Gamma_{w, r_{s}+f_{s}}^{+}\right)}^{2, \frac{\alpha}{2}}\left(\mathcal{N}_{r_{s}+f_{s}}^{+}\right)$. We will prove the existence part of Proposition 2 by constructing a continuous map of $\mathcal{P}\left(M_{1}\right)$ into itself as a map from $\mathbb{R} \times$ $C_{\left(-1-\frac{\alpha}{2}, \Gamma_{w, r_{s}+f_{s}}^{+}\right)}^{2, \frac{\alpha}{2}}\left(\mathcal{N}_{r_{s}+f_{s}}^{+}\right)$to $\mathbb{R} \times C_{\left(-1-\frac{\alpha}{2}, \Gamma_{w, r_{s}+f_{s}}^{+}\right)}^{2, \frac{\alpha}{2}}\left(\mathcal{N}_{r_{s}+f_{s}}^{+}\right)$and applying the Schauder fixed point theorem.

In this proof, $C$ s and $C_{i}$ for $i=1,2, \ldots$ denote positive constants depending on the whole or a part of the data, $\delta_{1}, \delta_{2}, \delta_{4}, \delta_{5}$ and $\delta_{6}$ unless otherwise specified. Each $C$ in different situations differs from each other.

1. For a fixed $\left(f(0), \Psi \mathbf{e}_{\varphi}\right)$, solve $\left(\mathrm{B}^{\prime}\right)$.

Take $\left(f(0)^{*}, \tilde{\Psi}^{*} \mathbf{e}_{\varphi}\right) \in \mathcal{P}\left(M_{1}\right)$. By the definition of $f_{s}$ and the assumption that $f_{s}^{\prime}$ satisfies (3.1),

$$
\left\|f_{S}\right\|_{2, \alpha, \Lambda}^{(-1-\alpha, \partial \Lambda)} \leq C_{1} \sigma
$$

Choose $\bar{\sigma}_{3}=\min \left(\frac{\delta_{1}}{2 M_{1}}, \frac{\delta_{1}}{2 C_{1}}\right)\left(=: \bar{\sigma}_{3}^{(1)}\right)$ so that

$$
\left|f(0)^{*}-r_{s}\right| \leq \frac{\delta_{1}}{2} \text { and }\left\|f_{s}\right\|_{2, \alpha, \Lambda}^{(-1-\alpha, \partial \Lambda)} \leq \frac{\delta_{1}}{2}
$$

Then, using $\Pi_{f(0)^{*}+f_{s} r_{s}+f_{s}}$, extend $\tilde{\Psi}^{*} \mathbf{e}_{\varphi}$ to a function in $\mathcal{N}_{f(0)^{*}+f_{s}}^{+}$:

$$
\Psi^{*} \mathbf{e}_{\varphi}:=\frac{W^{*}}{2 \pi r \sin \theta} \mathbf{e}_{\varphi},
$$

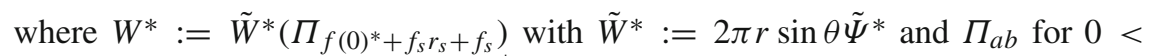
$a, b<r_{1}$ is a map from $\mathcal{N}_{a}^{+}$to $\mathcal{N}_{b}^{+}$defined in Step 1 in the proof of Lemma 10. By (3.91) and the fact that $\left\|\tilde{\Psi}^{*} \mathbf{e}_{\varphi}\right\|_{2, \alpha, \mathcal{N}_{r_{s}+f_{s}}^{+}}^{\left(-1-\alpha, \Gamma_{w, r_{s}+f_{s}}^{+}\right)} \leq M_{1} \sigma$,

$$
\left\|\nabla \times\left(\Psi^{*} \mathbf{e}_{\varphi}\right)\right\|_{1, \alpha, \mathcal{N}_{f(0)^{*}+f_{s}}^{+}}^{\left(-\alpha, \Gamma^{+}\right.}+C_{2} M_{1} \sigma .
$$


Choose $\bar{\sigma}_{3}=\min \left(\frac{\delta_{6}}{C_{2} M_{1}}, \bar{\sigma}_{3}^{(1)}\right)\left(=: \bar{\sigma}_{3}^{(2)}\right)$ where $\delta_{6}$ is a positive constant given in Lemma 15 so that $\Psi \mathbf{e}_{\varphi}=\Psi^{*} \mathbf{e}_{\varphi}$ satisfies (3.81) for $f=f(0)^{*}+f_{s}$.

For $\left(f(0), \Psi \mathbf{e}_{\varphi}\right)=\left(f(0)^{*}, \Psi^{*} \mathbf{e}_{\varphi}\right)$, solve $\left(\mathrm{B}^{\prime}\right)$ : solve

$$
\begin{aligned}
& \left\{\begin{array}{l}
\nabla \times\left(\left(\Phi_{0}^{+}+\Psi^{*}\right) \mathbf{e}_{\varphi}\right) \cdot \nabla A=0 \text { in } \mathcal{N}_{f(0)^{*}+f_{s}}^{+}, \\
A=A_{e n, f(0)^{*}+f_{s}} \text { on } \Gamma_{f(0)^{*}+f_{s},}
\end{array}\right. \\
& \left\{\begin{array}{l}
\nabla \times\left(\left(\Phi_{0}^{+}+\Psi^{*}\right) \mathbf{e}_{\varphi}\right) \cdot \nabla T=0 \text { in } \mathcal{N}_{f(0)^{*}+f_{s}}^{+}, \\
T=T_{e n, f(0)^{*}+f_{s}} \text { on } \Gamma_{f(0)^{*}+f_{s},}
\end{array}\right.
\end{aligned}
$$

where $A_{e n, f(0)^{*}+f_{s}}$ and $T_{e n, f(0)^{*}+f_{s}}$ are $A_{e n, f(0)+f_{s}}$ and $T_{e n, f(0)+f_{s}}$ given in (3.20) and (3.21), respectively, for $f(0)=f(0)^{*}$.

$$
\begin{aligned}
& \text { By }\left(\rho_{-}, \mathbf{u}_{-}, p_{-}, f_{s}^{\prime}\right) \in\left(C^{2, \alpha}(\overline{\mathcal{N}})\right)^{3} \times C_{\left(-\alpha,\left\{\theta=\theta_{1}\right\}\right), 0}^{1, \alpha}\left(\left(0, \theta_{1}\right)\right) \text {, } \\
& A_{e n, f(0)^{*}+f_{s}} \in C_{\left(-1-\alpha, \partial \Gamma_{f(0) *+f_{s}}\right)}^{2, \alpha}\left(\Gamma_{f(0)^{*}+f_{s}}\right) \\
& \text { and } T_{e n, f(0)^{*}+f_{s}} \in C_{\left(-\alpha, \partial \Gamma_{f(0)^{*}+f_{s}}^{1, \alpha}\right.}\left(\Gamma_{f(0)^{*}+f_{s}}\right) \text {. }
\end{aligned}
$$

Take $\bar{\sigma}_{3}=\min \left(\bar{\sigma}_{3}^{(2)}, \delta_{5}\right)\left(=: \bar{\sigma}_{3}^{(3)}\right)$ where $\delta_{5}$ is a positive constant given in Lemma 6 so that $\left(\rho_{-}, \mathbf{u}_{-}, p_{-}\right)$satisfies $(2.19)$ for $\sigma \leq \delta_{5}$. Then by Lemma $6,(3.1)$ and the fact that $f(0)^{*}$ satisfies $\left|f(0)^{*}-r_{s}\right| \leq M_{1} \sigma$,

$$
\left\|T_{e n, f^{*}}\right\|_{1, \alpha, \Gamma_{f^{*}}}^{\left(-\alpha, \partial \Gamma_{f^{*}}\right)} \leq C M_{1} \sigma+C \sigma
$$

where $f^{*}:=f^{*}(0)+f_{s}$. Apply Lemma 15 to (3.93), (3.94). Then we obtain that each (3.93) and (3.94) has a unique axisymmetric $C^{0}\left(\overline{\mathcal{N}_{f}^{+}}\right) \cap C^{1}\left(\mathcal{N}_{f}^{+}\right)$solution

$$
A^{*}=A_{e n, f(0)^{*}+f_{s}}\left(\mathcal{L}^{*}\right) \text { and } T^{*}=T_{e n, f(0)^{*}+f_{s}}\left(\mathcal{L}^{*}\right),
$$

respectively, where $\mathcal{L}^{*}$ is $\mathcal{L}$ defined in Lemma 15 for $V=2 \pi r \sin \theta\left(\Phi_{0}^{+}+\Psi^{*}\right)$ and $f=f(0)^{*}+f_{s}$. Furthermore, we have that $T^{*}$ satisfies

$$
\left\|T^{*}\right\|_{1, \alpha, \mathcal{N}_{f^{*}}^{+}}^{\left(-\alpha, \Gamma_{w, f^{*}}^{+}\right)} \leq C M_{1} \sigma+C \sigma,
$$

where we used (3.95). Using the solution expression of $A^{*}$, estimate $\frac{A^{*}}{2 \pi r \sin \theta} \mathbf{e}_{\varphi}$ in $C^{1, \alpha}\left(\overline{\mathcal{N}_{f^{*}}^{+}}\right)$. Using (3.20), write $\frac{A^{*}}{2 \pi r \sin \theta} \mathbf{e}_{\varphi}$ as

$$
\frac{2 \pi f^{*}\left(\mathcal{L}^{*}\right) \sin \left(\mathcal{L}^{*}\right) u_{-, \varphi}\left(\mathcal{L}^{*}\right)}{2 \pi r \sin \theta} \mathbf{e}_{\varphi},
$$

where $u_{-, \varphi}=\mathbf{u}_{-} \cdot \mathbf{e}_{\varphi}$. Using arguments similar to the ones in the proof of Claim in Lemma 15, we can obtain

$$
\left\|\frac{\mathcal{L}^{*}}{\theta}\right\|_{0, \alpha,\left(f^{*}(\theta), r_{1}\right) \times\left(0, \theta_{1}\right)} \leq C .
$$


With this estimate, $\left\|\mathcal{L}^{*}\right\|_{1, \alpha,\left(f^{*}(\theta), r_{1}\right) \times\left(0, \theta_{1}\right)} \leq C,(2.19)$ and (3.91), we estimate (3.97) in $C^{1, \alpha}\left(\overline{\mathcal{N}_{f^{*}}^{+}}\right)$. Then we have

$$
\left\|\frac{A^{*}}{2 \pi r \sin \theta} \mathbf{e}_{\varphi}\right\|_{1, \alpha, \mathcal{N}_{f^{*}}^{+}} \leq C \sigma
$$

2. By substituting an extension of $\left(\Psi^{*} \mathbf{e}_{\varphi}, A^{*}, T^{*}\right)$ into nonlinear parts of $\left(\mathrm{A}^{\prime}\right)$, obtain a linear problem having unknowns $\left(f(0), \Psi \mathbf{e}_{\varphi}\right)$.

Let $f(0)^{\sharp}$ be a point in $\left[r_{s}-\frac{\delta_{1}}{2}, r_{s}+\frac{\delta_{1}}{2}\right]$. By the choice of $\bar{\sigma}_{3}$,

$$
\left\|f^{\sharp}-r_{s}\right\|_{2, \alpha, \Lambda}^{(-1-\alpha, \partial \Lambda)} \leq \delta_{1},
$$

where $f^{\sharp}:=f(0)^{\sharp}+f_{s}$. Extend $\Psi^{*} \mathbf{e}_{\varphi} A^{*}$ and $T^{*}$ to functions in $\mathcal{N}_{f^{\sharp}}^{+}$:

$$
\Psi^{\sharp, *} \mathbf{e}_{\varphi}:=\frac{W^{\sharp, *}}{2 \pi r \sin \theta} \mathbf{e}_{\varphi}, \quad A^{\sharp, *}:=A^{*}\left(\Pi_{f^{\sharp} f^{*}}\right) \quad \text { and } \quad T^{\sharp, *}:=T^{*}\left(\Pi_{f^{\sharp} f^{*}}\right),
$$

where $W^{\sharp, *}=W^{*}\left(\Pi_{f^{\sharp} f^{*}}\right)$. By (3.92), (3.96) and (3.98),

$$
\begin{aligned}
\| \nabla & \times\left(\Psi^{\sharp, *} \mathbf{e}_{\varphi}\right)\left\|_{1, \alpha, \mathcal{N}_{f^{\sharp}}^{+}}^{\left(-\alpha, \Gamma_{w}^{\sharp}\right.}+\right\| \frac{A^{\sharp}, *}{2 \pi r \sin \theta} \mathbf{e}_{\varphi}\left\|_{1, \alpha, \mathcal{N}_{f^{\sharp}}^{+}}+\right\| T^{\sharp, *} \|_{1, \alpha, \mathcal{N}_{f^{\sharp}}^{+}}^{\left(-\alpha, \Gamma_{w}^{\sharp}\right)} \\
& \leq C_{3} M_{1} \sigma+C_{4} \sigma
\end{aligned}
$$

for all $f^{\sharp}(0) \in\left[r_{s}-\frac{\delta_{1}}{2}, r_{s}+\frac{\delta_{1}}{2}\right]$. Take $\bar{\sigma}_{3}=\min \left(\bar{\sigma}_{3}^{(3)}, \frac{\delta_{4}}{C_{3} M_{1}+C_{4}}\right)\left(=: \bar{\sigma}_{3}^{(4)}\right)$ where $\delta_{4}$ is a positive constant given in Lemma 5 so that $\varrho\left(\nabla \times\left(\left(\Phi_{0}^{+}+\Psi^{\sharp}, *\right) \mathbf{e}_{\varphi}\right)\right.$, $\left.\frac{A^{\sharp, *}}{2 \pi r \sin \theta} \mathbf{e}_{\varphi}, S_{0}^{+}+T^{\sharp, *}\right)$ is well-defined in $\mathcal{N}_{f^{\sharp}}^{+}$and $\varrho\left(\nabla \times\left(\left(\Phi_{0}^{+}+\Psi^{\sharp, *}\right) \mathbf{e}_{\varphi}\right)\right.$, $\left.\frac{A^{\sharp, *}}{2 \pi r \sin \theta} \mathbf{e}_{\varphi}, S_{0}^{+}+T^{\sharp, *}\right)$ and $S_{0}^{+}+T^{\sharp, *}$ are strictly positive in $\mathcal{N}_{f^{\sharp}}^{+}$for all $f^{\sharp}(0) \in$ $\left[r_{s}-\frac{\delta_{1}}{2}, r_{s}+\frac{\delta_{1}}{2}\right]$. By substituting $f(0)^{\sharp}$ and $\left(\Psi^{\sharp, *} \mathbf{e}_{\varphi}, A^{\sharp, *}, T^{\sharp, *}\right)$ into the place of $f(0)$ in $\left(\mathrm{A}^{\prime}\right)$ and $\left(\Psi \mathbf{e}_{\varphi}, A, T\right)$ in $\boldsymbol{F}_{1}\left(\Psi \mathbf{e}_{\varphi}, A, T\right), \mathfrak{f}_{0}\left(T, p_{e x}\right)$ and $\mathfrak{f}_{1}\left(\Psi \mathbf{e}_{\varphi}, A, T\right)$ in $\left(\mathrm{A}^{\prime}\right)$, we obtain

$$
\begin{aligned}
& \nabla \times\left(\frac{1}{\rho_{0}^{+}}\left(1+\frac{u_{0}^{+} \mathbf{e}_{r} \otimes u_{0}^{+} \mathbf{e}_{r}}{c_{0}^{+2}-u_{0}^{+2}}\right) \nabla \times\left(\Psi \mathbf{e}_{\varphi}\right)\right) \\
& =\frac{\rho_{0}^{+\gamma-1}}{(\gamma-1) u_{0}^{+}}\left(1+\frac{\gamma u_{0}^{+2}}{c_{0}^{+2}-u_{0}^{+2}}\right) \frac{\partial_{\theta} T}{r} \mathbf{e}_{\varphi} \\
& +\boldsymbol{F}_{1}\left(\Psi^{\sharp, *} \mathbf{e}_{\varphi}, A^{\sharp, *}, T^{\sharp, *}\right) \text { in } \mathcal{N}_{f^{\sharp}}^{+} \text {, } \\
& \Psi \mathbf{e}_{\varphi}=\left\{\begin{array}{l}
\left(\Phi_{-}-\Phi_{0}^{-}\right) \mathbf{e}_{\varphi} \text { on } \Gamma_{f^{\sharp}}, \\
\frac{r_{0}\left(\Phi_{-}-\Phi_{0}^{-}\right)\left(r_{0}, \theta_{1}\right)}{r} \mathbf{e}_{\varphi} \text { on } \Gamma_{w, f^{\sharp}}^{+}:=\Gamma_{w} \cap\left\{r>f^{\sharp}\right\}, \\
\left(\frac { 1 } { r _ { 1 } \operatorname { s i n } \theta } \int _ { 0 } ^ { \theta } \left(\mathfrak{f}_{0}\left(T^{\sharp, *}, p_{e x}\right)-\frac{\rho_{0}^{+}\left((\gamma-1) u_{0}^{+2}+c_{0}^{+2}\right)}{\gamma(\gamma-1) u_{0}^{+} S_{0}^{+}} T\right.\right. \\
\left.\left.+\mathfrak{f}_{1}\left(\Psi^{\sharp, *} \mathbf{e}_{\varphi}, A^{\sharp, *}, T^{\sharp, *}\right)\right) r_{1}^{2} \sin \xi \mathrm{d} \xi\right) \mathbf{e}_{\varphi} \text { on } \Gamma_{e x},
\end{array}\right.
\end{aligned}
$$




$$
\begin{aligned}
& \frac{1}{r_{1} \sin \theta_{1}} \int_{0}^{\theta_{1}}\left(\mathfrak{f}_{0}\left(T^{\sharp, *}, p_{e x}\right)-\frac{\rho_{0}^{+}\left((\gamma-1) u_{0}^{+2}+c_{0}^{+2}\right)}{\gamma(\gamma-1) u_{0}^{+} S_{0}^{+}} T\right. \\
& \left.+\mathfrak{f}_{1}\left(\Psi^{\sharp, *} \mathbf{e}_{\varphi}, A^{\sharp, *}, T^{\sharp, *}\right)\right)\left.\right|_{r=r_{1}} r_{1}^{2} \sin \xi \mathrm{d} \xi=\frac{r_{0}\left(\Phi_{-}-\Phi_{0}^{-}\right)\left(r_{0}, \theta_{1}\right)}{r_{1}} .
\end{aligned}
$$

We let $f(0)^{\sharp}$ be an unknown in this problem and let $T$ in (3.100)-(3.102) be a solution of

$$
\left\{\begin{array}{l}
\nabla \times\left(\left(\Phi_{0}^{+}+\Psi^{\sharp}, *\right) \mathbf{e}_{\varphi}\right) \cdot \nabla T=0 \text { in } \mathcal{N}_{f^{\sharp}}^{+}, \\
T=T_{e n, f^{\sharp}} \text { on } \quad \Gamma_{f^{\sharp}} .
\end{array}\right.
$$

Then since a solution $T$ of (3.103) is uniquely determined by $f(0)^{\sharp}$ (see Step 3), unknowns of (3.100)-(3.102) become $\left(f^{\sharp}(0), \Psi \mathbf{e}_{\varphi}\right)$. We denote an unknown $\Psi \mathbf{e}_{\varphi}$ of (3.100)-(3.102) by $\Psi^{\sharp} \mathbf{e}_{\varphi}$.

3. Find $f(0)^{\sharp}$ using (3.102).

By the definition of $\Psi^{\sharp, *} \mathbf{e}_{\varphi}$ and (2.27), the transport equation in (3.103) can be written as

$$
\nabla^{\perp}\left(V_{0}^{+}+W^{\sharp, *}\right) \cdot \nabla T=0 \text { in } \mathcal{N}_{f^{\sharp}}^{+} .
$$

From this form of the transport equation in (3.103), we see that the stream surface of the vector field $\nabla \times\left(\left(\Phi_{0}^{+}+\Psi^{\sharp, *}\right) \mathbf{e}_{\varphi}\right)$ in $\mathcal{N}_{f^{\sharp}}^{+}$is obtained by stretching or contracting the stream surface of the vector field $\nabla \times\left(\left(\Phi_{0}^{+}+\Psi^{*}\right) \mathbf{e}_{\varphi}\right)$ in $\mathcal{N}_{f^{*}}^{+}$in $r$-direction. Using this fact, we obtain that the solution of (3.103) is given by

$$
T=T_{e n, f}\left(\mathcal{L}^{\sharp}\right),
$$

where $\mathcal{L}^{\sharp}=\mathcal{L}^{*}\left(\Pi_{f^{\sharp} f^{*}}\right)$. We denote this solution by $T^{\sharp}$.

By (3.21), $T^{\sharp}$ is expressed as

$$
T^{\sharp}=\left(g\left(\left(\frac{\mathbf{u}_{-} \cdot \boldsymbol{v}_{f^{\sharp}}\left(\mathcal{L}^{\sharp}\right)}{c_{-}}\right)^{2}\right) S_{-}\right)\left(f(0)^{\sharp}+f_{s}\left(\mathcal{L}^{\sharp}\right), \mathcal{L}^{\sharp}\right)-\left(g\left(M_{0}^{-2}\right)\right)\left(r_{s}\right) S_{\text {in }} .
$$

Substituting this expression of $T^{\sharp}$ into the place of $T$ in (3.102) using the fact that $\Pi_{f^{\sharp} f^{*}}\left(r_{1}, \theta\right)=\left(r_{1}, \theta\right)$, we obtain

$$
\begin{aligned}
(L):= & \left.\frac{1}{r_{1} \sin \theta_{1}} \int_{0}^{\theta_{1}} \frac{\rho_{0}^{+}\left((\gamma-1) u_{0}^{+2}+c_{0}^{+2}\right)}{\gamma(\gamma-1) u_{0}^{+} S_{0}^{+}}\right|_{r=r_{1}}(a) r_{1} r_{1}^{2} \sin \xi \mathrm{d} \xi \\
= & -\frac{r_{0}\left(\Phi_{-}-\Phi_{0}^{-}\right)\left(r_{0}, \theta_{1}\right)}{r_{1}}+\frac{1}{r_{1} \sin \theta_{1}} \int_{0}^{\theta_{1}}\left(\mathfrak{f}_{0}\left(T^{*}, p_{e x}\right)\right. \\
& \left.-\frac{\rho_{0}^{+}\left((\gamma-1) u_{0}^{+^{2}}+c_{0}^{+^{2}}\right)}{\gamma(\gamma-1) u_{0}^{+} S_{0}^{+}}\left((a)_{2}+(a)_{3}\right)+\mathfrak{f}_{1}\left(\Psi^{*} \mathbf{e}_{\varphi}, A^{*}, T^{*}\right)\right)\left.\right|_{r=r_{1}} \\
& r_{1}^{2} \sin \xi \mathrm{d} \xi \\
= & :(R)
\end{aligned}
$$


where

$$
\begin{aligned}
(a)_{1}= & \left(g\left(M_{0}^{-2}\right)\right)\left(f(0)^{\sharp}+f_{s}\left(\mathcal{L}^{*}\left(r_{1}, \theta\right)\right)\right) S_{i n} \\
& -\left(g\left(M_{0}^{-2}\right)\right)\left(r_{s}+f_{s}\left(\mathcal{L}^{*}\left(r_{1}, \theta\right)\right)\right) S_{i n}, \\
(a)_{2}= & \left(g\left(\left(\frac{\mathbf{u}_{-} \cdot \boldsymbol{v}_{f^{\sharp}}\left(\mathcal{L}^{*}\left(r_{1}, \theta\right)\right)}{c_{-}}\right)^{2}\right) S_{-}\right)\left(f(0)^{\sharp}+f_{s}\left(\mathcal{L}^{*}\left(r_{1}, \theta\right)\right), \mathcal{L}^{*}\left(r_{1}, \theta\right)\right) \\
& -\left(g\left(M_{0}^{-2}\right)\right)\left(f(0)^{\sharp}+f_{s}\left(\mathcal{L}^{*}\left(r_{1}, \theta\right)\right)\right) S_{i n}, \\
(a)_{3}= & \left(g\left(M_{0}^{-2}\right)\right)\left(r_{s}+f_{s}\left(\mathcal{L}^{*}\left(r_{1}, \theta\right)\right)\right) S_{i n}-\left(g\left(M_{0}^{-2}\right)\right)\left(r_{s}\right) S_{i n} .
\end{aligned}
$$

We find $f(0)^{\sharp}$ satisfying (3.104). For this, we estimate $|(R)|$.

Estimate $|(R)|$ : have

With (2.19) and (3.1), we estimate $(a)_{2},(a)_{3}$ and $\frac{r_{0}\left(\Phi_{-}-\Phi_{0}^{-}\right)\left(r_{0}, \theta_{1}\right)}{r_{1}}$. Then we

$$
\begin{aligned}
\sup _{\theta \in\left(0, \theta_{1}\right)}\left|(a)_{2}\right| & \leq C \sigma\left|f(0)^{\sharp}-r_{s}\right|+C \sigma \\
& \leq C \frac{\delta_{1}}{2} \sigma+C \sigma
\end{aligned}
$$

for all $f^{\sharp}(0) \in\left[r_{s}-\frac{\delta_{1}}{2}, r_{s}+\frac{\delta_{1}}{2}\right]$,

$$
\sup _{\theta \in\left(0, \theta_{1}\right)}\left|(a)_{3}\right| \leq C \sigma
$$

and

$$
\left|\frac{r_{0}\left(\Phi_{-}-\Phi_{0}^{-}\right)\left(r_{0}, \theta_{1}\right)}{r_{1}}\right| \leq C \sigma .
$$

With (2.20), we estimate $\mathfrak{f}_{0}\left(T^{*}, p_{e x}\right)\left(r_{1}, \theta\right)$. Then we have

$$
\sup _{\theta \in\left(0, \theta_{1}\right)}\left|\mathfrak{f}_{0}\left(T^{*}, p_{e x}\right)\left(r_{1}, \theta\right)\right| \leq C \sigma .
$$

By Lemma 5 and using (3.92), (3.96) and (3.98), we estimate $\mathfrak{f}_{1}\left(\Psi^{*} \mathbf{e}_{\varphi}, A^{*}, T^{*}\right)$ $\left(r_{1}, \theta\right)$. Then we have

$$
\sup _{\theta \in\left(0, \theta_{1}\right)}\left|\mathfrak{f}_{1}\left(\Psi^{*} \mathbf{e}_{\varphi}, A^{*}, T^{*}\right)\left(r_{1}, \theta\right)\right| \leq C\left(M_{1}+1\right)^{2} \sigma^{2} .
$$

With (3.105)-(3.109), we estimate $|(R)|$. Then we obtain

$$
|(R)| \leq C_{5}\left(M_{1}+1\right)^{2} \sigma^{2}+C_{6} \sigma
$$

for all $f^{\sharp}(0) \in\left[r_{s}-\frac{\delta_{1}}{2}, r_{s}+\frac{\delta_{1}}{2}\right]$.

Then we find $f(0)^{\sharp}$. By Lemma 2 , there exists a positve constant $\lambda$ such that

$$
(L)^{\prime}\left(f(0)^{\sharp}\right) \geq \lambda
$$


for all $f^{\sharp}(0) \in\left[r_{s}-\frac{\delta_{1}}{2}, r_{s}+\frac{\delta_{1}}{2}\right]$. By this fact and $(L)\left(r_{s}\right)=0$,

$$
\text { (L) }\left(\frac{\delta_{1}}{2}\right) \geq \frac{\delta_{1} \lambda}{2} \text { and (L) }\left(-\frac{\delta_{1}}{2}\right) \leq-\frac{\delta_{1} \lambda}{2} \text {. }
$$

Choose $\bar{\sigma}_{3}=\min \left(\bar{\sigma}_{3}^{(4)}, \frac{C_{6}}{C_{5}\left(M_{1}+1\right)^{2}}, \frac{\delta_{1} \lambda}{8 C_{6}}\right)\left(=: \bar{\sigma}_{3}^{(5)}\right)$. Then since

$$
|(R)| \leq \frac{\delta_{1} \lambda}{4}
$$

for all $f(0)^{\sharp} \in\left[r_{s}-\frac{\delta_{1}}{2}, r_{s}+\frac{\delta_{1}}{2}\right]$ and (3.111) holds, by the intermediate value theorem, there exists $f(0)^{\sharp}$ satisfying (3.104) in $\left[r_{s}-\frac{\delta_{1}}{2}, r_{s}+\frac{\delta_{1}}{2}\right]$. Such $f(0)^{\sharp}$ is unique because $(L)$ is a monotone function of $f(0)^{\sharp}$. Since at $f(0)^{\sharp}$ where (3.104) holds it holds that

$$
\lambda\left|f(0)^{\sharp}-r_{s}\right| \leq\left|(R)\left(f(0)^{\sharp}\right)\right|,
$$

we have that

$$
\left|f(0)^{\sharp}-r_{s}\right| \leq C\left(M_{1}+1\right)^{2} \sigma^{2}+C \sigma .
$$

\section{Find $\Psi^{\sharp} \mathbf{e}_{\varphi}$.}

Fix $f(0)^{\sharp}$ obtained in Step 3. By (3.112), Lemma 6 and Lemma 15, $T^{\sharp}$ determined by $f(0)^{\sharp}$ satisfies

$$
\left\|T^{\sharp}\right\|_{1, \alpha, \mathcal{N}_{f^{\sharp}}^{+}}^{\left(-\alpha, \Gamma_{w}^{\sharp}\right)} \leq C\left(M_{1}+1\right)^{2} \sigma^{2}+C \sigma .
$$

Substitute this $T^{\sharp}$ into the place of $T$ in (3.100) and (3.101). Then we obtain

$$
\begin{aligned}
& \nabla \times\left(\frac{1}{\rho_{0}^{+}}\left(1+\frac{u_{0}^{+} \mathbf{e}_{r} \otimes u_{0}^{+} \mathbf{e}_{r}}{c_{0}^{+2}-u_{0}^{+2}}\right) \nabla \times\left(\Psi \mathbf{e}_{\varphi}\right)\right) \\
& =\frac{\rho_{0}^{+\gamma-1}}{(\gamma-1) u_{0}^{+}}\left(1+\frac{\gamma u_{0}^{+2}}{c_{0}^{+2}-u_{0}^{+2}}\right) \frac{\partial_{\theta} T^{\sharp}}{r} \mathbf{e}_{\varphi}+\boldsymbol{F}_{1}\left(\Psi^{\sharp, *} \mathbf{e}_{\varphi}, A^{\sharp, *}, T^{\sharp, *}\right) \text { in } \mathcal{N}_{f^{\sharp}}^{+} \text {, } \\
& \Psi \mathbf{e}_{\varphi}=\left\{\begin{array}{l}
\left(\Phi_{-}-\Phi_{0}^{-}\right) \mathbf{e}_{\varphi} \text { on } \Gamma_{f^{\sharp}}, \\
\frac{r_{0}\left(\Phi_{-}-\Phi_{0}^{-}\right)\left(r_{0}, \theta_{1}\right)}{r} \mathbf{e}_{\varphi} \text { on } \Gamma_{w, f^{\sharp}}^{+}, \\
\left(\frac { 1 } { r _ { 1 } \operatorname { s i n } \theta } \int _ { 0 } ^ { \theta } \left(\mathfrak{f}_{0}\left(T^{\sharp, *}, p_{e x}\right)-\frac{\rho_{0}^{+}\left((\gamma-1) u_{0}^{+2}+c_{0}^{+2}\right)}{\gamma(\gamma-1) u_{0}^{+} S_{0}^{+}} T^{\sharp}\right.\right. \\
\left.\left.\quad+\mathfrak{f}_{1}\left(\Psi^{\sharp, *} \mathbf{e}_{\varphi}, A^{\sharp, *}, T^{\sharp, *}\right)\right) r_{1}^{2} \sin \xi \mathrm{d} \xi\right) \mathbf{e}_{\varphi} \text { on } \Gamma_{e x} .
\end{array}\right.
\end{aligned}
$$

Since $f(0)^{\sharp}$ is chosen for $T=T^{\sharp}$ to satisfy (3.102), (3.115) is a continuous boundary condition. We apply Lemma 7 to (3.114), (3.115) with (2.19), (2.20), (3.1), 
(3.99) and (3.113). Then we obtain that (3.114), (3.115) has a unique $C_{\left(-1-\alpha, \Gamma_{f} \sharp\right)}^{2, \alpha}$ $\left(\mathcal{N}_{f^{\sharp}}^{+}\right)$solution $\Psi \sharp \mathbf{e}_{\varphi}$ and this solution satisfies the estimate

$$
\left\|\Psi^{\sharp} \mathbf{e}_{\varphi}\right\|_{2, \alpha, \mathcal{N}_{f^{\sharp}}^{+}}^{\left(-1-\alpha, \Gamma_{w, f^{\sharp}}^{+}\right)} \leq C\left(M_{1}+1\right)^{2} \sigma^{2}+C \sigma .
$$

5. Using $\Pi_{r_{s}+f_{s} f^{\sharp}}$, transform $\Psi^{\sharp} \mathbf{e}_{\varphi}$ into a function in $\mathcal{N}_{r_{s}+f_{s}}^{+}$:

$$
\tilde{\Psi}^{\sharp} \mathbf{e}_{\varphi}=\frac{\tilde{W}^{\sharp}}{2 \pi r \sin \theta} \mathbf{e}_{\varphi},
$$

where $\tilde{W}^{\sharp}=W^{\sharp}\left(\Pi_{r_{s}+f_{s} f^{\sharp}}\right)$ with $W^{\sharp}=2 \pi r \sin \theta \Psi^{\sharp}$. By (3.112) and (3.116),

$$
\left\|\tilde{\Psi}^{\sharp} \mathbf{e}_{\varphi}\right\|_{2, \alpha, \mathcal{N}_{r_{s}+f_{s}}^{+}}^{\left(-1-\alpha, \Gamma_{w, r_{s}+f_{s}}^{+}\right)} \leq C\left(M_{1}+1\right)^{2} \sigma^{2}+C \sigma .
$$

Combining this with (3.112), we have

$$
\left|f(0)^{\sharp}-r_{s}\right|+\left\|\tilde{\Psi}^{\sharp} \mathbf{e}_{\varphi}\right\|_{2, \alpha, \mathcal{N}_{r_{s}+f_{s}}^{+}}^{\left(-1-\alpha, \Gamma_{w, r_{s}+f_{s}}^{+}\right)} \leq C_{7}\left(M_{1}+1\right)^{2} \sigma^{2}+C_{8} \sigma .
$$

Take $M_{1}=2 C_{8}$ and $\bar{\sigma}_{3}=\min \left(\bar{\sigma}_{3}^{(5)}, \frac{C_{8}}{C_{7}\left(M_{1}+1\right)^{2}}\right)\left(=: \bar{\sigma}_{3}^{(6)}\right)$. And then define a $\operatorname{map} \mathcal{J}$ from $\mathbb{R} \times C_{\left(-1-\frac{\alpha}{2}, \Gamma_{w, r_{s}+f_{s}}^{+}\right)}^{2, \frac{\alpha}{2}}\left(\mathcal{N}_{r_{s}+f_{s}}^{+}\right)$to $\mathbb{R} \times C_{\left(-1-\frac{\alpha}{2}, \Gamma_{w, r_{s}+f_{s}}^{+}\right)}^{2, \frac{\alpha}{2}}\left(\mathcal{N}_{r_{s}+f_{s}}^{+}\right)$by

$$
\mathcal{J}\left(f(0)^{*}, \tilde{\Psi}^{*} \mathbf{e}_{\varphi}\right)=\left(f(0)^{\sharp}, \tilde{\Psi}^{\sharp} \mathbf{e}_{\varphi}\right) .
$$

Then by the choice of $M_{1}$ and $\bar{\sigma}_{3}, \mathcal{J}$ is a map of $\mathcal{P}\left(M_{1}\right)$ into itself. Using the standard argument, one can easily check that $\mathcal{J}$ is continuous. Thus, the Schauder fixed point theorem can be applied to $\mathcal{J}$. We apply the Schauder fixed point theorem to $\mathcal{J}$. Then we obtain that there exists a fixed point $\left(f^{b}(0), \tilde{\Psi}^{b} \mathbf{e}_{\varphi}\right) \in \mathcal{P}\left(M_{1}\right)$ of $\mathcal{J}$.

One can see that if $\left(f(0)^{*}, \tilde{\Psi}^{*} \mathbf{e}_{\varphi}\right)=\left(f(0)^{\sharp}, \tilde{\Psi}^{\sharp} \mathbf{e}_{\varphi}\right)$, then $\Psi^{*} \mathbf{e}_{\varphi}=\Psi^{\sharp}, \mathbf{e}_{\varphi}=$ $\Psi^{\sharp} \mathbf{e}_{\varphi}, A^{\sharp, *}=A^{*}$ and $T^{*}=T^{\sharp, *}=T^{\sharp}$. From this fact, we see that $\left(f(0), \Phi \mathbf{e}_{\varphi}, L\right.$, $S)=\left(f(0)^{b},\left(\Phi_{0}^{+}+\Psi^{b}\right) \mathbf{e}_{\varphi}, A^{b}, S_{0}^{+}+T^{b}\right)$, where

$$
\Psi^{b} \mathbf{e}_{\varphi}:=\frac{\left(2 \pi r \sin \theta \tilde{\Psi}^{b}\right)\left(\Pi_{f(0)^{b}+f_{s} r_{s}+f_{s}}\right)}{2 \pi r \sin \theta} \mathbf{e}_{\varphi}
$$

and $A^{b}$ and $T^{b}$ are solutions of (3.93) and (3.94) for given $\left(f(0)^{*}, \Psi^{*}\right)=\left(f(0)^{b}, \Psi^{b}\right)$, respectively, is a solution of (A), (B). By $\left(f(0)^{b}, \tilde{\Psi}^{b} \mathbf{e}_{\varphi}\right) \in \mathcal{P}\left(M_{1}\right)$, (3.96) and (3.98),

$$
\begin{aligned}
& \left|f(0)^{b}-r_{s}\right| \\
& +\left\|\nabla \times\left(\Psi^{b} \mathbf{e}_{\varphi}\right)\right\|_{1, \alpha, \mathcal{N}_{f^{b}}^{+}}^{\left(-\alpha, \Gamma_{w, f^{b}}^{+}\right)}+\left\|\frac{A^{b}}{2 \pi r \sin \theta}\right\|_{1, \alpha, \mathcal{N}_{f^{b}}^{+}} \\
& +\left\|T^{b}\right\|_{1, \alpha, \mathcal{N}_{f^{b}}^{+}}^{\left(-\alpha, \Gamma^{+}\right.}+C \sigma,
\end{aligned}
$$


where $f^{b}:=f(0)^{b}+f_{s}$. One can easily see that there exists a positive constant $\delta_{7}$ such that for any $\mathcal{N}_{f}^{+} \subset \mathcal{N}_{r_{s}-\delta_{1}}^{+}$, if $\left(\nabla \times\left(\Phi \mathbf{e}_{\varphi}\right), \frac{L}{2 \pi r \sin \theta} \mathbf{e}_{\varphi}, S, B_{0}\right) \in B_{\delta_{7}, \mathcal{N}_{f}^{+}}^{(1)}$ where $B_{\delta, \Omega}^{(1)}$ for $\delta>0$ and $\Omega \subset \mathbb{R}^{3}$ is a neighborhood of $\left(\nabla \times\left(\Phi_{0}^{+} \mathbf{e}_{\varphi}\right), 0, S_{0}^{+}, B_{0}\right)$ defined in Lemma 3, then (2.51) holds in $\mathcal{N}_{f}^{+}$. Let $\delta_{7}$ be one such constant. Take $\bar{\sigma}_{3}=$ $\min \left(\bar{\sigma}_{3}^{(6)}, \frac{\delta_{7}}{C}\right)$ where $C$ is $C$ in (3.117). Then $\left(f(0)^{b},\left(\Phi_{0}^{+}+\Psi^{b}\right) \mathbf{e}_{\varphi}, A^{b}, S_{0}^{+}+T^{b}\right)$ is a subsonic solution of (2.43)-(2.45) in $\mathcal{N}_{f^{b}}^{+}$. Choose $\delta_{1}, \delta_{2}, \delta_{4}, \delta_{5}, \delta_{6}$ and $\delta_{7}$ so that they can depend on the data. Then $M_{1}$ and $\bar{\sigma}_{3}$ depend on the data. This finishes the proof.

Proof of Proposition 2 (Uniqueness) Let $\alpha \in\left(\frac{2}{3}, 1\right.$ ). Let $\sigma_{3}$ be a positive constant $\leq \bar{\sigma}_{3}$ and to be determined later. Suppose that there exist two solutions $\left(f_{i}(0), \Phi_{i} \mathbf{e}_{\varphi}, L_{i}, S_{i}\right)$ for $i=1,2$ of Problem 3 for $\sigma \leq \sigma_{3}$ satisfying the estimate (3.2).

Let $\left(\Psi_{i}, A_{i}, T_{i}\right):=\left(\Phi_{i}-\Phi_{0}^{+}, L_{i}, S_{i}-S_{0}^{+}\right)$for $i=1,2$. We will prove that there exists a positive constant $\underline{\sigma}_{3}$ such that if $\sigma_{3}=\underline{\sigma}_{3}$, then

$$
\left(f_{1}(0), \Psi_{1} \mathbf{e}_{\varphi}, A_{1}, T_{1}\right)=\left(f_{2}(0), \Psi_{2} \mathbf{e}_{\varphi}, A_{2}, T_{2}\right),
$$

by constructing a contraction map in a low regularity space.

In this proof, $C$ s denote positive constants depending on the data unless otherwise specified. Each $C$ in different situations differs from each other.

1. By subtracting $\left(\mathrm{A}^{\prime}\right)$ satisfied by $\left(f_{1}(0), \Psi_{1} \mathbf{e}_{\varphi}, A_{1}, T_{1}\right)$ from $\left(\mathrm{A}^{\prime}\right)$ satisfied by $\left(f_{2}(0), \Psi_{2} \mathbf{e}_{\varphi}, A_{2}, T_{2}\right)$, obtain the equations that will give a contraction map.

Let $f_{i}:=f_{i}(0)+f_{s}$ for $i=1,2$. From

$$
\left(\nabla^{\perp} a\right)\left(\Pi_{f_{2} f_{1}}\right)=N \nabla^{\perp}\left(a\left(\Pi_{f_{2} f_{1}}\right)\right) \text { and } \nabla \times\left(\frac{a \mathbf{e}_{\varphi}}{2 \pi r \sin \theta}\right)=\nabla^{\perp} a
$$

for an axisymmetric scalar function $a$, we can obtain

$$
\begin{aligned}
& \left(\nabla \times\left(\Psi_{1} \mathbf{e}_{\varphi}\right)\right)\left(\Pi_{f_{2} f_{1}}\right)=N \nabla \times\left(\tilde{\Psi}_{1} \mathbf{e}_{\varphi}\right) \\
& \text { and }\left(\nabla \times\left(\frac{A_{1}}{2 \pi r \sin \theta} \mathbf{e}_{\varphi}\right)\right)\left(\Pi_{f_{2} f_{1}}\right)=N \nabla \times\left(\frac{\tilde{A}_{1}}{2 \pi r \sin \theta} \mathbf{e}_{\varphi}\right)
\end{aligned}
$$

where $\tilde{\Psi}_{1}:=\frac{\tilde{W}_{1}}{2 \pi r \sin \theta}$ with $\tilde{W}_{1}:=W_{1}\left(\Pi_{f_{2} f_{1}}\right)$ and $W_{1}:=2 \pi r \sin \theta \Psi_{1}, \tilde{A}_{1}:=$ $A_{1}\left(\Pi_{f_{2} f_{1}}\right)$ and

$$
\begin{aligned}
N= & \frac{r^{2}}{\left(\Pi_{f_{2} f_{1}}^{r}\right)^{2}} \mathbf{e}_{r} \otimes \mathbf{e}_{r}-\frac{\left(\partial_{\tilde{\theta}} \Pi_{f_{1} f_{2}}^{r}\right)\left(\Pi_{f_{2} f_{1}}\right)}{\left(\Pi_{f_{2} f_{1}}^{r}\right)^{2}} r \mathbf{e}_{r} \otimes \mathbf{e}_{\theta} \\
& +\frac{\left(\partial_{\tilde{r}} \Pi_{f_{1} f_{2}}^{r}\right)\left(\Pi_{f_{2} f_{1}}\right)}{\Pi_{f_{2} f_{1}}^{r}} r \mathbf{e}_{\theta} \otimes \mathbf{e}_{\theta},
\end{aligned}
$$

with $\Pi_{f_{2} f_{1}}^{r}$ and $\Pi_{f_{1} f_{2}}^{r}, r$-components of $\Pi_{f_{2} f_{1}}^{*}$ and $\Pi_{f_{1} f_{2}}^{*}$, respectively (see the definition of $\Pi_{a b}^{*}$ in $\left.(3.56)\right),(\tilde{r}, \tilde{\theta}),(r, \theta)$ coordinates for the Cartesian coordinate for $\mathcal{N}_{f_{1}(0)+f_{s}}^{+}$, and $(r, \theta)=\Pi_{f_{1} f_{2}}^{*}(\tilde{r}, \tilde{\theta})$. Using $\Pi_{f_{2} f_{1}}$ and (3.118), transform $\left(\mathrm{A}^{\prime}\right)$ 
satisfied by $\left(f_{1}(0), \Psi_{1} \mathbf{e}_{\varphi}, A_{1}, T_{1}\right)$. And then subtract the resultant equations from (A') satisfied by $\left(f_{2}(0), \Psi_{2} \mathbf{e}_{\varphi}, A_{2}, T_{2}\right)$. Then we obtain

$$
\begin{aligned}
& \nabla \times\left(\frac{1}{\rho_{0}^{+}}\left(1+\frac{u_{0}^{+} \mathbf{e}_{r} \otimes u_{0}^{+} \mathbf{e}_{r}}{c_{0}^{+2}-u_{0}^{+2}}\right) \nabla \times\left(\left(\Psi_{2}-\tilde{\Psi}_{1}\right) \mathbf{e}_{\varphi}\right)\right) \\
& =\frac{\rho_{0}^{+\gamma-1}}{(\gamma-1) u_{0}^{+}}\left(1+\frac{\gamma u_{0}^{+2}}{c_{0}^{+2}-u_{0}^{+2}}\right) \frac{\partial_{\theta}\left(T_{2}-\tilde{T}_{1}\right)}{r} \mathbf{e}_{\varphi}+\boldsymbol{F}_{3} \text { in } \mathcal{N}_{f_{2}(0)+f_{s}}^{+} \\
& \left(\Psi_{2}-\tilde{\Psi}_{1}\right) \mathbf{e}_{\varphi} \\
& \left(\left(\Phi_{-}-\Phi_{0}^{-}\right) \mathbf{e}_{\varphi}-\frac{\Pi_{f_{2} f_{1}}^{r}\left(\Phi_{-}-\Phi_{0}^{-}\right)\left(\Pi_{f_{2} f_{1}}\right)}{r} \mathbf{e}_{\varphi}\left(=: \overline{\boldsymbol{h}}_{1}\right) \text { on } \Gamma_{f_{2}(0)+f_{s}},\right. \\
& \mathbf{0} \text { on } \Gamma_{w, f_{2}(0)+f_{s}}^{+}:=\Gamma_{w} \cap\left\{r>f_{2}(0)+f_{s}\right\} \text {, } \\
& =\left\{\left(\frac { 1 } { r _ { 1 } \operatorname { s i n } \theta } \int _ { 0 } ^ { \theta } \left(\mathfrak{f}_{0}\left(T_{2}, p_{e x}\right)-\mathfrak{f}_{0}\left(\tilde{T}_{1}, p_{e x}\right)-\frac{\rho_{0}^{+}\left((\gamma-1) u_{0}^{+2}+c_{0}^{+2}\right)}{\gamma(\gamma-1) u_{0}^{+} S_{0}^{+}}\left(T_{2}-\tilde{T}_{1}\right)\right.\right.\right. \\
& \left.\left.+\mathfrak{f}_{1}\left(\Psi_{2} \mathbf{e}_{\varphi}, A_{2}, T_{2}\right)-\mathfrak{f}_{1}\left(\tilde{\Psi}_{1} \mathbf{e}_{\varphi}, \tilde{A}_{1}, \tilde{T}_{1}\right)\right) r_{1}^{2} \sin \xi \mathrm{d} \xi\right) \mathbf{e}_{\varphi}\left(=: \overline{\boldsymbol{h}}_{2}\right) \\
& \text { on } \Gamma_{e x} \text {, } \\
& 0=\frac{1}{r_{1} \sin \theta_{1}} \int_{0}^{\theta_{1}}\left(\mathfrak{f}_{0}\left(T_{2}, p_{e x}\right)-\mathfrak{f}_{0}\left(\tilde{T}_{1}, p_{e x}\right)-\frac{\rho_{0}^{+}\left((\gamma-1) u_{0}^{+2}+c_{0}^{+2}\right)}{\gamma(\gamma-1) u_{0}^{+} S_{0}^{+}}\right. \\
& \times\left(T_{2}-\tilde{T}_{1}\right) \\
& \left.+\mathfrak{f}_{1}\left(\Psi_{2} \mathbf{e}_{\varphi}, A_{2}, T_{2}\right)-\mathfrak{f}_{1}\left(\tilde{\Psi}_{1} \mathbf{e}_{\varphi}, \tilde{A}_{1}, \tilde{T}_{1}\right)\right)\left.\right|_{r=r_{1}} r_{1}^{2} \sin \xi \mathrm{d} \xi,
\end{aligned}
$$

where

$$
\begin{aligned}
\boldsymbol{F}_{3}= & -\nabla \times\left(\frac{1}{\rho_{0}^{+}}\left(1+\frac{u_{0}^{+} \mathbf{e}_{r} \otimes u_{0}^{+} \mathbf{e}_{r}}{c_{0}^{+2}-u_{0}^{+2}}\right) \nabla \times\left(\tilde{\Psi}_{1} \mathbf{e}_{\varphi}\right)\right) \\
& +M \nabla \times\left(\left(\frac{1}{\rho_{0}^{+}}\left(1+\frac{u_{0}^{+} \mathbf{e}_{r} \otimes u_{0}^{+} \mathbf{e}_{r}}{c_{0}^{+^{2}}-u_{0}^{+2}}\right)\right)\left(\Pi_{f_{2} f_{1}}\right) N \nabla \times\left(\tilde{\Psi}_{1} \mathbf{e}_{\varphi}\right)\right) \\
& +\frac{\rho_{0}^{+\gamma-1}}{(\gamma-1) u_{0}^{+}}\left(1+\frac{\gamma u_{0}^{+2}}{c_{0}^{+2}-u_{0}^{+2}}\right) \frac{1}{r} \partial_{\theta} \tilde{T}_{1} \mathbf{e}_{\varphi} \\
& -\left(\frac{\rho_{0}^{+\gamma-1}}{(\gamma-1) u_{0}^{+}}\left(1+\frac{\gamma u_{0}^{+2}}{c_{0}^{+2}-u_{0}^{+2}}\right) \frac{1}{r}\right)\left(\Pi_{f_{2} f_{1}}\right)\left(\frac{\partial \Pi_{f_{1} f_{2}}^{r}}{\partial \tilde{\theta}}\left(\Pi_{f_{2} f_{1}}\right) \partial_{r}+\partial_{\theta}\right) \tilde{T}_{1} \mathbf{e}_{\varphi} \\
& +\boldsymbol{F}_{1}\left(\Psi_{2} \mathbf{e}_{\varphi}, A_{2}, T_{2}\right)-\tilde{\boldsymbol{F}}_{1}\left(\tilde{\Psi}_{1} \mathbf{e}_{\varphi}, \tilde{A}_{1}, \tilde{T}_{1}\right), \\
\tilde{T}_{1}= & T_{1}\left(\Pi_{f_{2} f_{1}}\right),
\end{aligned}
$$




$$
\begin{aligned}
M= & \left(\partial_{\tilde{r}} \Pi_{f_{1} f_{2}}^{r}\right)\left(\Pi_{f_{2} f_{1}}\right) \mathbf{e}_{r} \otimes \mathbf{e}_{r}+\frac{\left(\partial_{\tilde{\theta}} \Pi_{f_{1} f_{2}}^{r}\right)\left(\Pi_{f_{2} f_{1}}\right)}{\Pi_{f_{2} f_{1}}^{r}} \mathbf{e}_{\theta} \otimes \mathbf{e}_{r} \\
& +\frac{r}{\Pi_{f_{2} f_{1}}^{r}} \mathbf{e}_{\theta} \otimes \mathbf{e}_{\theta}+\frac{r}{\Pi_{f_{2} f_{1}}^{r}} \mathbf{e}_{\varphi} \otimes \mathbf{e}_{\varphi},
\end{aligned}
$$

and $\tilde{\boldsymbol{F}}_{1}$ is $\boldsymbol{F}_{1}$ changed by using the transformation $\Pi_{f_{2} f_{1}}$. We will construct a contraction map using (3.119)-(3.121). For this, we estimate $\left\|T_{2}-\tilde{T}_{1}\right\|_{0, \beta, \mathcal{N}_{f_{2}}^{+}}$for $\beta=1-\frac{3}{q} \in(0, \alpha)$ where $q \in\left(3, \frac{1}{1-\alpha}\right)$ and $\left\|\left(\frac{A_{2}}{2 \pi r \sin \theta}-\frac{\tilde{A}_{1}}{2 \pi r \sin \theta}\right) \mathbf{e}_{\varphi}\right\|_{1,0, \mathcal{N}_{f_{2}}^{+}}$.

2. Estimate $\left\|T_{2}-\tilde{T}_{1}\right\|_{0, \beta, \mathcal{N}_{f_{2}}^{+}}$.

Since $T_{i}$ for $i=1,2$ are solutions of $\nabla \times\left(\left(\Phi_{0}^{+}+\Psi_{i}\right) \mathbf{e}_{\varphi}\right) \cdot \nabla T=0$ in $\mathcal{N}_{f_{i}(0)+f_{s}}^{+}, \quad T=T_{e n, f_{i}(0)+f_{s}}$ on $\Gamma_{f_{i}(0)+f_{s}}$ for $i=1,2$, respectively, where $T_{e n, f_{i}(0)+f_{s}}$ is $T_{e n, f(0)+f_{s}}$ given in (3.21) for $f(0)=f_{i}(0)$, by Lemma 15 ,

$$
T_{i}=T_{e n, f_{i}(0)+f_{s}}\left(\mathcal{L}_{i}\right)
$$

for $i=1,2$ where $\mathcal{L}_{i}$ are $\mathcal{L}$ given in Lemma 15 for $V=V_{0}^{+}+W_{i}\left(=: V_{i}\right)$ and $f=f_{i}(0)+f_{s}$ with $W_{i}:=2 \pi r \sin \theta \Psi_{i}$.

By (3.21), (3.122) and the definition of $\tilde{T}_{1}, T_{2}-\tilde{T}_{1}$ can be written as

$$
\begin{aligned}
& \left(g\left(\left(\frac{\mathbf{u}_{-} \cdot \boldsymbol{v}_{f_{2}}\left(\mathcal{L}_{2}\right)}{c_{-}}\right)^{2}\right) S_{-}\right)\left(f_{2}\left(\mathcal{L}_{2}\right), \mathcal{L}_{2}\right) \\
& \quad-\left(g\left(\left(\frac{\mathbf{u}_{-} \cdot \boldsymbol{v}_{f_{1}}\left(\tilde{\mathcal{L}}_{1}\right)}{c_{-}}\right)^{2}\right) S_{-}\right)\left(f_{1}\left(\tilde{\mathcal{L}}_{1}\right), \tilde{\mathcal{L}}_{1}\right)
\end{aligned}
$$

where $\boldsymbol{v}_{f_{i}}$ for $i=1,2$ are the unit normal vectors on $\Gamma_{f_{i}}$ pointing toward $\mathcal{N}_{f_{i}}^{+}$, respectively, and $\tilde{\mathcal{L}}_{1}:=\mathcal{L}_{1}\left(\Pi_{f_{2} f_{1}}\right)$. This can be decomposed into $(a)+(b)$ where

$$
\begin{aligned}
(a):= & \left(g\left(\left(\frac{\mathbf{u}_{-} \cdot \boldsymbol{v}_{f_{2}}\left(\mathcal{L}_{2}\right)}{c_{-}}\right)^{2}\right) S_{-}\right)\left(f_{2}\left(\mathcal{L}_{2}\right), \mathcal{L}_{2}\right) \\
& -\left(g\left(\left(\frac{\mathbf{u}_{-} \cdot \boldsymbol{v}_{f_{1}}\left(\tilde{\mathcal{L}}_{1}\right)}{c_{-}}\right)^{2}\right) S_{-}\right)\left(f_{2}\left(\mathcal{L}_{2}\right), \mathcal{L}_{2}\right)
\end{aligned}
$$

and

$$
\begin{aligned}
(b):= & \left(g\left(\left(\frac{\mathbf{u}_{-} \cdot \boldsymbol{v}_{f_{1}}\left(\tilde{\mathcal{L}}_{1}\right)}{c_{-}}\right)^{2}\right) S_{-}\right)\left(f_{2}\left(\mathcal{L}_{2}\right), \mathcal{L}_{2}\right) \\
& -\left(g\left(\left(\frac{\mathbf{u}_{-} \cdot \boldsymbol{v}_{f_{1}}\left(\tilde{\mathcal{L}}_{1}\right)}{c_{-}}\right)^{2}\right) S_{-}\right)\left(f_{1}\left(\tilde{\mathcal{L}}_{1}\right), \tilde{\mathcal{L}}_{1}\right) .
\end{aligned}
$$


To estimate $T_{2}-\tilde{T}_{1}$ in $C^{\beta}\left(\overline{\mathcal{N}_{f_{2}}^{+}}\right)$, we estimate $(a)$ and $(b)$ in $C^{\beta}\left(\overline{\mathcal{N}_{f_{2}}^{+}}\right)$, respectively. To obtain an estimate of $(a)$ in $C^{\beta}\left(\overline{\mathcal{N}_{f_{2}}^{+}}\right)$and later to obtain an estimate of

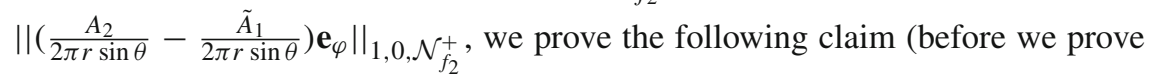
the Claim, we take $\sigma_{3}=\min \left(\bar{\sigma}_{3}, \frac{\delta_{6}}{C}\right)\left(=: \sigma_{3}^{(1)}\right)$ where $C$ is $C$ in (3.2) so that $\Psi \mathbf{e}_{\varphi}=\Psi_{i} \mathbf{e}_{\varphi}$ for $i=1,2$ satisfy (3.79) for $\left.f=f_{i}(0)+f_{s}\right)$ :

Claim. Let $h:\left[0, \theta_{1}\right] \rightarrow \mathbb{R}$ be a function in $C_{\left(-\alpha,\left\{\theta=\theta_{1}\right\}\right)}^{1, \alpha}\left(\left(0, \theta_{1}\right)\right)$. It holds that

$$
\begin{aligned}
& \left\|\int_{0}^{1} h^{\prime}\left(t \mathcal{L}_{2}+(1-t) \tilde{\mathcal{L}}_{1}\right) \mathrm{d} t\left(\mathcal{L}_{2}-\tilde{\mathcal{L}}_{1}\right)\right\|_{0, \beta, \mathcal{N}_{f_{2}}^{+}} \\
& \quad \leq C\|h\|_{1, \beta,\left(0, \theta_{1}\right)}^{\left(-\alpha,\left\{\theta=\theta_{1}\right\}\right)}\left\|\left(\Psi_{2}-\tilde{\Psi}_{1}\right) \mathbf{e}_{\varphi}\right\|_{1, \beta, \mathcal{N}_{f_{2}}^{+}} .
\end{aligned}
$$

Proof of Claim. By the definitions of $\mathcal{L}_{2}$ and $\tilde{\mathcal{L}}_{1}$,

$$
\mathcal{L}_{2}=k_{2}^{-1} \circ V_{2} \text { and } \tilde{\mathcal{L}}_{1}=\left(k_{1}^{-1} \circ V_{1}\right)\left(\Pi_{f_{2} f_{1}}\right)
$$

where $k_{i}(\theta)=V_{i}\left(f_{i}(\theta), \theta\right)$ for $i=1,2$. By $V_{0}^{+}\left(\Pi_{f_{2} f_{1}}\right)=V_{0}^{+}$and $\tilde{W}_{1}\left(f_{2}(\theta), \theta\right)=$ $W_{1}\left(f_{1}(\theta), \theta\right)$ where $\tilde{W}_{1}$ is defined below (3.118), $\tilde{\mathcal{L}}_{1}$ can be written as

$$
\tilde{\mathcal{L}}_{1}=\tilde{k}_{1}^{-1} \circ \tilde{V}_{1}
$$

where $\tilde{V}_{1}=V_{0}^{+}+\tilde{W}_{1}$ and $\tilde{k}_{1}=\tilde{V}_{1}\left(f_{2}(\theta), \theta\right)$. Since $\tilde{V}_{1}(r, \theta) \in\left[0, V_{-}\left(r_{0}, \theta_{1}\right)\right]$ for $(r, \theta) \in\left[f_{2}(\theta), r_{1}\right] \times\left[0, \theta_{1}\right]$ and $V_{2}\left(f_{2}(\theta), \theta\right) \in\left[0, V_{-}\left(r_{0}, \theta_{1}\right)\right]$ for $\theta \in\left[0, \theta_{1}\right]$ where $V_{-}=2 \pi r \sin \theta \Phi_{-}, k_{2}^{-1} \circ \tilde{V}_{1}$ is well-defined in $\overline{\mathcal{N}_{f_{2}}^{+}}$. With this fact, we write $\mathcal{L}_{2}-\tilde{\mathcal{L}}_{1}$ as

$$
\begin{aligned}
\mathcal{L}_{2}-\tilde{\mathcal{L}}_{1}= & \left(k_{2}^{-1} \circ V_{2}-k_{2}^{-1} \circ \tilde{V}_{1}\right)+\left(k_{2}^{-1} \circ \tilde{V}_{1}-\tilde{k}_{1}^{-1} \circ \tilde{V}_{1}\right) \\
= & \int_{0}^{1} \frac{1}{k_{2}^{\prime}\left(k_{2}^{-1} \circ\left(t V_{2}+(1-t) \tilde{V}_{1}\right)\right)} \mathrm{d} t\left(V_{2}-\tilde{V}_{1}\right) \\
& +\int_{0}^{1} \frac{1}{\tilde{k}_{1}^{\prime}\left(\tilde{k}_{1}^{-1} \circ\left(t \tilde{k}_{1}(\vartheta)+(1-t) k_{2}(\vartheta)\right)\right)} \mathrm{d} t\left(\tilde{k}_{1}(\vartheta)-k_{2}(\vartheta)\right),
\end{aligned}
$$

where $\vartheta=k_{2}^{-1} \circ \tilde{V}_{1}$ and we used the fact that $k_{2}^{-1} \circ \tilde{V}_{1}-\tilde{k}_{1}^{-1} \circ \tilde{V}_{1}=\tilde{k}_{1}^{-1} \circ \tilde{k}_{1}(\vartheta)-$ $\tilde{k}_{1}^{-1} \circ k_{2}(\vartheta)$.

Substitute the above expression of $\mathcal{L}_{2}-\tilde{\mathcal{L}}_{1}$ into the place of $\mathcal{L}_{2}-\tilde{\mathcal{L}}_{1}$ in $\int_{0}^{1} h^{\prime}\left(t \mathcal{L}_{2}+(1-t) \tilde{\mathcal{L}}_{1}\right) \mathrm{d} t\left(\mathcal{L}_{2}-\tilde{\mathcal{L}}_{1}\right)$. Then we have

$$
\begin{aligned}
\int_{0}^{1} & h^{\prime}\left(t \mathcal{L}_{2}+(1-t) \tilde{\mathcal{L}}_{1}\right) \mathrm{d} t\left(\mathcal{L}_{2}-\tilde{\mathcal{L}}_{1}\right) \\
= & \int_{0}^{1} h^{\prime}\left(t \mathcal{L}_{2}+(1-t) \tilde{\mathcal{L}}_{1}\right) \mathrm{d} t \int_{0}^{1} \frac{1}{k_{2}^{\prime}\left(k_{2}^{-1} \circ\left(t V_{2}+(1-t) \tilde{V}_{1}\right)\right)} \mathrm{d} t\left(V_{2}-\tilde{V}_{1}\right) \\
& +\int_{0}^{1} h^{\prime}\left(t \mathcal{L}_{2}+(1-t) \tilde{\mathcal{L}}_{1}\right) \mathrm{d} t \int_{0}^{1} \frac{1}{\tilde{k}_{1}^{\prime}\left(\tilde{k}_{1}^{-1} \circ\left(t \tilde{k}_{1}(\vartheta)+(1-t) k_{2}(\vartheta)\right)\right)} \mathrm{d} t \\
& \left(\tilde{k}_{1}(\vartheta)-k_{2}(\vartheta)\right)=:(c)+(d) .
\end{aligned}
$$


To estimate $\left\|\int_{0}^{1} h^{\prime}\left(t \mathcal{L}_{2}+(1-t) \tilde{\mathcal{L}}_{1}\right) \mathrm{d} t\left(\mathcal{L}_{2}-\tilde{\mathcal{L}}_{1}\right)\right\|_{0, \beta, \mathcal{N}_{f_{2}}^{+}}$, we estimate $(c)$ and $(d)$ in $C^{\beta}\left(\overline{\mathcal{N}_{f_{2}}^{+}}\right)$, respectively.

Estimate $(c)$ in $C^{\beta}\left(\overline{\mathcal{N}_{f_{2}}^{+}}\right)$:

With the definitions of $k_{2}, \Psi_{2}$ and $\tilde{\Psi}_{1}$, write $(c)$ as

$$
\begin{aligned}
(c)= & \int_{0}^{1}\left(\theta-\theta_{1}\right) h^{\prime}\left(t \mathcal{L}_{2}+(1-t) \tilde{\mathcal{L}}_{1}\right) \mathrm{d} t \\
& \int_{0}^{1} \frac{r \sin \theta}{J \sin \left(k_{2}^{-1} \circ\left(t V_{2}+(1-t) \tilde{V}_{1}\right)\right)} \mathrm{d} t \int_{0}^{1} \partial_{\theta} \\
& \left(\Psi_{2}-\tilde{\Psi}_{1}\right)\left(r, t \theta+(1-t) \theta_{1}\right) \mathrm{d} t,
\end{aligned}
$$

where

$$
\begin{aligned}
J:= & \left(f_{2}^{2} \sqrt{1+\left(\frac{f_{2}^{\prime}}{f_{2}}\right)^{2}}\right)(\cdot) \\
& \left.\left(\nabla \times\left(\left(\Phi_{0}^{+}+\Psi_{2}\right) \mathbf{e}_{\varphi}\right)\right)(f(\cdot), \cdot) \cdot \boldsymbol{v}_{f_{2}}(\cdot)\right|_{.=k_{2}^{-1} \circ\left(t V_{2}+(1-t) \tilde{V}_{1}\right)} .
\end{aligned}
$$

By the choice of $\sigma_{3}, \Psi \mathbf{e}_{\varphi}=\Psi_{i} \mathbf{e}_{\varphi}$ for $i=1,2$ satisfy (3.79) for $f=f_{i}(0)+f_{s}$. By this fact and $\left\|f_{2}-r_{s}\right\|_{2, \alpha, \Lambda}^{(-1-\alpha, \partial \Lambda)} \leq \delta_{1}$ and the fact that $k_{2}^{-1} \circ\left(t V_{2}+(1-t) \tilde{V}_{1}\right)$ $\operatorname{maps} \overline{\mathcal{N}_{f_{2}}^{+}}$to $\left[0, \theta_{1}\right]$,

$$
J>\tilde{c}^{*} \text { in } \mathcal{N}_{f_{2}}^{+}
$$

for all $t \in[0,1]$ for some positive constant $\tilde{c}^{*}$. Using arguments similar to the ones in the proof of Claim in Lemam 15, we can obtain

$$
\left\|\frac{\theta-\theta_{1}}{\left(t \mathcal{L}_{2}+(1-t) \tilde{\mathcal{L}}_{1}\right)-\theta_{1}}\right\|_{0, \beta,\left(f_{2}(\theta), r_{1}\right) \times\left(0, \theta_{1}\right)} \leq C
$$

and

$$
\left\|\frac{\theta}{k_{2}^{-1} \circ\left(t V_{2}+(1-t) \tilde{V}_{1}\right)}\right\|_{0, \beta,\left(f_{2}(\theta), r_{1}\right) \times\left(0, \theta_{1}\right)} \leq C
$$

for any $t \in[0,1]$. With (3.124)-(3.126), $\|a b\|_{0, \beta, \Omega} \leq\|a\|_{0, \beta, \Omega}\|b\|_{0,0, \Omega}+$ $\|a\|_{0,0, \Omega}\|b\|_{0, \beta, \Omega},(3.2)$ satisfied by $\left(f_{i}(0), \Phi_{i} \mathbf{e}_{\varphi}, L_{i}, S_{i}\right)$ for $i=1,2$,

$$
\begin{aligned}
h^{\prime} \in C_{\left(1-\alpha,\left\{\theta=\theta_{1}\right\}\right)}^{\alpha}\left(\left(0, \theta_{1}\right)\right), & \\
& \quad\left\|\mathcal{L}_{2}\right\|_{1,0,\left(f_{2}(\theta), r_{1}\right) \times\left(0, \theta_{1}\right)} \leq C,\left\|\tilde{\mathcal{L}}_{1}\right\|_{1,0,\left(f_{2}(\theta), r_{1}\right) \times\left(0, \theta_{1}\right)} \leq C
\end{aligned}
$$

and $\left\|k_{2}^{-1} \circ\left(t V_{2}+(1-t) \tilde{V}_{1}\right)\right\|_{1,0,\left(f_{2}(\theta), r_{1}\right) \times\left(0, \theta_{1}\right)} \leq C$, we estimate the right hand side of (3.123) in $C^{\beta}\left(\overline{\mathcal{N}_{f_{2}}^{+}}\right)$. Then we obtain

$$
\left.\|(c)\|\right|_{0, \beta, \mathcal{N}_{f_{2}}^{+}} \leq C\|h\|_{1, \beta,\left(0, \theta_{1}\right)}^{\left(-\alpha,\left\{\theta=\theta_{1}\right\}\right)}\left\|\left(\Psi_{2}-\tilde{\Psi}_{1}\right) \mathbf{e}_{\varphi}\right\|_{1, \beta, \mathcal{N}_{f_{2}}^{+}} .
$$


(d) can be estimated in $C^{\beta}\left(\overline{\mathcal{N}_{f_{2}}^{+}}\right)$in a similar way. As we do this, $\tilde{k}_{1}$ and $k_{2}$ play the role of $V_{2}$ and $\tilde{V}_{1}$ in the estimate of $(e)$ in $C^{\beta}\left(\overline{\mathcal{N}_{f_{2}}^{+}}\right)$and $\vartheta$ is regarded as the argument of $\tilde{k}_{1}$ and $k_{2}$.

We have

$$
\|(d)\|_{0, \beta, \mathcal{N}_{f_{2}}^{+}} \leq C\|h\|_{1, \beta,\left(0, \theta_{1}\right)}^{\left(-\alpha,\left\{\theta=\theta_{1}\right\}\right)}\left\|\left(\Psi_{2}-\tilde{\Psi}_{1}\right) \mathbf{e}_{\varphi}\right\|_{1, \beta, \mathcal{N}_{f_{2}}^{+}} .
$$

Combining (3.128) and (3.129), we obtain the desired result.

Note that if we change $\mathcal{L}_{2}-\tilde{\mathcal{L}}_{1}$ in the way that we changed $\mathcal{L}_{2}-\tilde{\mathcal{L}}_{1}$ in the estimate of $\int_{0}^{1} h^{\prime}\left(t \mathcal{L}_{2}+(1-t) \tilde{\mathcal{L}}_{1}\right) \mathrm{d} t\left(\mathcal{L}_{2}-\tilde{\mathcal{L}}_{1}\right)$ in the proof of Claim and estimate the resultant terms in $C^{\beta}\left(\overline{\mathcal{N}_{f_{2}}^{+}}\right)$without changing $V_{2}-\tilde{V}_{1}$ and $\tilde{k}_{1}(\vartheta)-k_{2}(\vartheta)$ into $2 \pi r \sin \theta\left(\theta-\theta_{1}\right) \int_{0}^{1} \partial_{\theta}\left(\Psi_{2}-\tilde{\Psi}_{1}\right)\left(r, t \theta+(1-t) \theta_{1}\right) \mathrm{d} t$ and $2 \pi f_{2}(\vartheta) \sin \vartheta(\vartheta-$ $\left.\theta_{1}\right)\left.\int_{0}^{1} \partial_{\theta}\left(\left(\tilde{\Psi}_{1}-\Psi_{2}\right)\left(f_{2}(\theta), \theta\right)\right)\right|_{\theta=t \vartheta+(1-t) \theta_{1}} \mathrm{~d} t$, then we obtain

$$
\left\|\mathcal{L}_{2}-\tilde{\mathcal{L}}_{1}\right\|_{0, \beta, \mathcal{N}_{f_{2}}^{+}} \leq C\left\|\left(\Psi_{2}-\tilde{\Psi}_{1}\right) \mathbf{e}_{\varphi}\right\|_{0, \beta, \mathcal{N}_{f_{2}}^{+}}
$$

With the above Claim, we estimate $(a)$ in $C^{\beta}\left(\overline{\mathcal{N}_{f_{2}}^{+}}\right)$. Write $(a)$ as

$$
\begin{aligned}
\int_{0}^{1} & \left(g^{\prime}\left(\left(\frac{\mathbf{u}_{-}}{c_{-}} \cdot\left(t \boldsymbol{v}_{f_{2}}\left(\mathcal{L}_{2}\right)+(1-t) \boldsymbol{v}_{f_{1}}\left(\tilde{\mathcal{L}}_{1}\right)\right)\right)^{2}\right)\right. \\
2 & \left.\left(\frac{\mathbf{u}_{-}}{c_{-}} \cdot\left(t \boldsymbol{v}_{f_{2}}\left(\mathcal{L}_{2}\right)+(1-t) \boldsymbol{v}_{f_{1}}\left(\tilde{\mathcal{L}}_{1}\right)\right)\right) \frac{\mathbf{u}_{-}}{c_{-}} S_{-}\right)\left(f_{2}\left(\mathcal{L}_{2}\right), \mathcal{L}_{2}\right) \mathrm{d} t \\
& \cdot\left(\boldsymbol{v}_{f_{2}}\left(\mathcal{L}_{2}\right)-\boldsymbol{v}_{f_{1}}\left(\tilde{\mathcal{L}}_{1}\right)\right) .
\end{aligned}
$$

By $\boldsymbol{v}_{f_{i}}=\frac{\mathbf{e}_{r}-\frac{f_{s}^{\prime}}{f_{i}(0)+f_{s}} \mathbf{e}_{\theta}}{\sqrt{1+\left(\frac{f_{s}^{\prime}}{f_{i}(0)+f_{s}}\right)^{2}}}$ for $i=1,2, \boldsymbol{v}_{f_{2}}\left(\mathcal{L}_{2}\right)-\boldsymbol{v}_{f_{1}}\left(\tilde{\mathcal{L}}_{1}\right)$ can written as

$$
t_{1} \mathbf{e}_{r}+t_{2} \mathbf{e}_{\theta}
$$

where

$$
\begin{aligned}
t_{1}= & \left.\int_{0}^{1} \nabla_{\left(f(0), f_{s}, f_{s}^{\prime}\right)}\left(\frac{1}{\sqrt{1+\left(\frac{f_{s}^{\prime}}{f(0)+f_{s}}\right)^{2}}}\right)\right|_{\left(f(0), f_{s}, f_{s}^{\prime}\right)=\chi} \mathrm{d} t \\
& \cdot\left(f_{2}(0)-f_{1}(0), f_{s}\left(\mathcal{L}_{2}\right)-f_{s}\left(\tilde{\mathcal{L}}_{1}\right), f_{s}^{\prime}\left(\mathcal{L}_{2}\right)-f_{s}^{\prime}\left(\tilde{\mathcal{L}}_{1}\right)\right)
\end{aligned}
$$

and

$$
\begin{aligned}
t_{2}= & \left.\int_{0}^{1} \nabla_{\left(f(0), f_{s}, f_{s}^{\prime}\right)}\left(\frac{-\frac{f_{s}^{\prime}}{f(0)+f_{s}}}{\sqrt{1+\left(\frac{f_{s}^{\prime}}{f(0)+f_{s}}\right)^{2}}}\right)\right|_{\left(f(0), f_{s}, f_{s}^{\prime}\right)=\chi} \mathrm{d} t \\
& \cdot\left(f_{2}(0)-f_{1}(0), f_{s}\left(\mathcal{L}_{2}\right)-f_{s}\left(\tilde{\mathcal{L}}_{1}\right), f_{s}^{\prime}\left(\mathcal{L}_{2}\right)-f_{s}^{\prime}\left(\tilde{\mathcal{L}}_{1}\right)\right)
\end{aligned}
$$


with $\chi=\left(t f_{2}(0)+(1-t) f_{1}(0), t f_{s}\left(\mathcal{L}_{2}\right)+(1-t) f_{s}\left(\tilde{\mathcal{L}}_{1}\right), t f_{s}^{\prime}\left(\mathcal{L}_{2}\right)+(1-t) f_{s}^{\prime}\left(\tilde{\mathcal{L}}_{1}\right)\right)$. To estimate $(3.131)$ in $C^{\beta}\left(\overline{\mathcal{N}_{f_{2}}^{+}}\right)$, we estimate $f_{s}\left(\mathcal{L}_{2}\right)-f_{s}\left(\tilde{\mathcal{L}}_{1}\right)$ and $f_{s}^{\prime}\left(\mathcal{L}_{2}\right)-f_{s}^{\prime}\left(\tilde{\mathcal{L}}_{1}\right)$ in $C^{\beta}\left(\overline{\mathcal{N}_{f_{2}}^{+}}\right)$, respectively.

Express $f_{s}\left(\mathcal{L}_{2}\right)-f_{s}\left(\tilde{\mathcal{L}}_{1}\right)$ and $f_{s}^{\prime}\left(\mathcal{L}_{2}\right)-f_{s}^{\prime}\left(\tilde{\mathcal{L}}_{1}\right)$ as

$$
\int_{0}^{1} f_{s}^{\prime}\left(t \mathcal{L}_{2}+(1-t) \mathcal{L}_{1}\right) \mathrm{d} t\left(\mathcal{L}_{2}-\tilde{\mathcal{L}}_{1}\right)
$$

and

$$
\int_{0}^{1} f_{s}^{\prime \prime}\left(t \mathcal{L}_{2}+(1-t) \tilde{\mathcal{L}}_{1}\right) \mathrm{d} t\left(\mathcal{L}_{2}-\tilde{\mathcal{L}}_{1}\right),
$$

respectively. Since $f_{s}^{\prime} \in C^{\alpha}\left(\left[0, \theta_{1}\right]\right),(3.133)$ can be estimated in $C^{\beta}\left(\overline{\mathcal{N}_{f_{2}}^{+}}\right)$directly. With (3.1), (3.127) and (3.130), we estimate (3.133) in $C^{\beta}\left(\overline{\mathcal{N}_{f_{2}}^{+}}\right)$directly. Then we obtain

$$
\left\|f_{s}\left(\mathcal{L}_{2}\right)-f_{s}\left(\tilde{\mathcal{L}}_{1}\right)\right\|_{0, \beta, \mathcal{N}_{f_{2}}^{+}} \leq C \sigma\left\|\left(\Psi_{2}-\tilde{\Psi}_{1}\right) \mathbf{e}_{\varphi}\right\|_{0, \beta, \mathcal{N}_{f_{2}}^{+}}
$$

Since $f_{s}^{\prime \prime} \in C_{\left(1-\alpha,\left\{\theta=\theta_{1}\right\}\right)}^{\alpha}\left(\left(0, \theta_{1}\right)\right)$, we cannot estimate (3.134) in $C^{\beta}\left(\overline{\mathcal{N}_{f_{2}}^{+}}\right)$directly. With Claim and (3.1), we estimate (3.134) in $C^{\beta}\left(\overline{\mathcal{N}_{f_{2}}^{+}}\right)$. Then we obtain

$$
\left\|f_{s}^{\prime}\left(\mathcal{L}_{2}\right)-f_{s}^{\prime}\left(\tilde{\mathcal{L}}_{1}\right)\right\|_{0, \beta, \mathcal{N}_{f_{2}}^{+}} \leq C \sigma\left\|\left(\Psi_{2}-\tilde{\Psi}_{1}\right) \mathbf{e}_{\varphi}\right\|_{1, \beta, \mathcal{N}_{f_{2}}^{+}}
$$

With (2.19), (3.1), (3.127), the expression of $\boldsymbol{v}_{f_{2}}\left(\mathcal{L}_{2}\right)-\boldsymbol{v}_{f_{1}}\left(\tilde{\mathcal{L}}_{1}\right)$ given in (3.132), (3.135) and (3.136), we estimate (3.131) in $C^{\beta}\left(\overline{\mathcal{N}_{f_{2}}^{+}}\right)$. Then we have

$$
\|(a)\|_{0, \beta, \mathcal{N}_{f_{2}}^{+}} \leq C \sigma\left|f_{2}(0)-f_{1}(0)\right|+C \sigma\left\|\left(\Psi_{2}-\tilde{\Psi}_{1}\right) \mathbf{e}_{\varphi}\right\|_{1, \beta, \mathcal{N}_{f_{2}}^{+}}
$$

Next, we estimate $(b)$ in $C^{\beta}\left(\overline{\mathcal{N}_{f_{2}}^{+}}\right)$. Divide $(b)$ into two parts:

$$
\begin{aligned}
& \left(g\left(\left(\frac{\mathbf{u}_{-} \cdot \boldsymbol{v}_{f_{1}}\left(\tilde{\mathcal{L}}_{1}\right)}{c_{-}}\right)^{2}\right) S_{-}\right)\left(f_{2}\left(\mathcal{L}_{2}\right), \mathcal{L}_{2}\right) \\
& \quad-\left(g\left(\left(\frac{\mathbf{u}_{-} \cdot \boldsymbol{v}_{f_{1}}\left(\tilde{\mathcal{L}}_{1}\right)}{c_{-}}\right)^{2}\right) S_{-}\right)\left(f_{2}\left(\tilde{\mathcal{L}}_{1}\right), \tilde{\mathcal{L}}_{1}\right)=:(b)_{1}
\end{aligned}
$$

and

$$
\begin{aligned}
& \left(g\left(\left(\frac{\mathbf{u}_{-} \cdot \boldsymbol{v}_{f_{1}}\left(\tilde{\mathcal{L}}_{1}\right)}{c_{-}}\right)^{2}\right) S_{-}\right)\left(f_{2}\left(\tilde{\mathcal{L}}_{1}\right), \tilde{\mathcal{L}}_{1}\right) \\
& \quad-\left(g\left(\left(\frac{\mathbf{u}_{-} \cdot \boldsymbol{v}_{f_{1}}\left(\tilde{\mathcal{L}}_{1}\right)}{c_{-}}\right)^{2}\right) S_{-}\right)\left(f_{1}\left(\tilde{\mathcal{L}}_{1}\right), \tilde{\mathcal{L}}_{1}\right)=:(b)_{2} .
\end{aligned}
$$


Write $(b)_{1}$ and $(b)_{2}$ as

$$
\left.\int_{0}^{1} \partial_{\theta}\left(g\left(\left(\frac{\mathbf{u}_{-} \cdot \boldsymbol{v}_{f_{1}}\left(\tilde{\mathcal{L}}_{1}\right)}{c_{-}}\right)^{2}\right) S_{-}\right)\left(f_{2}(\theta), \theta\right)\right|_{\theta=t \mathcal{L}_{2}+(1-t) \tilde{\mathcal{L}}_{1}} \mathrm{~d} t\left(\mathcal{L}_{2}-\tilde{\mathcal{L}}_{1}\right)
$$

and

$$
\begin{aligned}
& \left.\int_{0}^{1} \partial_{r}\left(g\left(\left(\frac{\mathbf{u}_{-} \cdot \boldsymbol{v}_{f_{1}}\left(\tilde{\mathcal{L}}_{1}\right)}{c_{-}}\right)^{2}\right) S_{-}\right)\left(r, \tilde{\mathcal{L}}_{1}\right)\right|_{r=t f_{2}\left(\tilde{\mathcal{L}}_{1}\right)+(1-t) f_{1}\left(\tilde{\mathcal{L}}_{1}\right)} \mathrm{d} t \\
& \quad\left(f_{2}(0)-f_{1}(0)\right) .
\end{aligned}
$$

Integrands in both the expressions are in $C^{\beta}\left(\overline{\mathcal{N}_{f_{2}}^{+}}\right)$. Thus, $(b)_{1}$ and $(b)_{2}$ can be estimated in $C^{\beta}\left(\overline{\mathcal{N}_{f_{2}}^{+}}\right)$directly. With (2.19), (3.1), (3.127) and (3.130), we estiamte $(b)_{1}$ and $(b)_{2}$ in $C^{\beta}\left(\overline{\mathcal{N}_{f_{2}}^{+}}\right)$, respectively. Then we obtain

$$
\left\|(b)_{1}\right\|_{0, \beta, \mathcal{N}_{f_{2}}^{+}} \leq C \sigma\left\|\left(\Psi_{2}-\tilde{\Psi}_{1}\right) \mathbf{e}_{\varphi}\right\|_{0, \beta, \mathcal{N}_{f_{2}}^{+}}
$$

and

$$
\left\|(b)_{2}\right\|_{0, \beta, \mathcal{N}_{f_{2}}^{+}} \leq C\left|f_{2}(0)-f_{1}(0)\right| .
$$

Combining (3.138) and (3.139), we have

$$
\|(b)\|_{0, \beta, \mathcal{N}_{f_{2}}^{+}} \leq C\left|f_{2}(0)-f_{1}(0)\right|+C \sigma\left\|\left(\Psi_{2}-\tilde{\Psi}_{1}\right) \mathbf{e}_{\varphi}\right\|_{0, \beta, \mathcal{N}_{f_{2}}^{+}}
$$

Finally, combine (3.137) and (3.140). Then we have

$$
\left\|T_{2}-\tilde{T}_{1}\right\|_{0, \beta, \mathcal{N}_{f_{2}}^{+}} \leq C\left|f_{2}(0)-f_{1}(0)\right|+C \sigma\left\|\left(\Psi_{2}-\tilde{\Psi}_{1}\right) \mathbf{e}_{\varphi}\right\|_{1, \beta, \mathcal{N}_{f_{2}}^{+}}
$$

3. Estimate $\left\|\left(\frac{A_{2}}{2 \pi r \sin \theta}-\frac{\tilde{A}_{1}}{2 \pi r \sin \theta}\right) \mathbf{e}_{\varphi}\right\|_{1,0, \mathcal{N}_{f_{2}}^{+}}$.

Since $A_{i}$ for $i=1,2$ are solutions of

$$
\nabla \times\left(\left(\Phi_{0}^{+}+\Psi_{i}\right) \mathbf{e}_{\varphi}\right) \cdot \nabla A=0 \text { in } \mathcal{N}_{f_{i}(0)+f_{s}}^{+}, A=A_{e n, f_{i}(0)+f_{s}} \text { on } \Gamma_{f_{i}(0)+f_{s}}
$$

for $i=1,2$, respectively, where $A_{e n, f_{i}}$ is $A_{e n, f(0)+f_{s}}$ given in (3.20) for $f(0)=$ $f_{i}(0)$, by Lemma 15 ,

$$
A_{i}=A_{e n, f_{i}}\left(\mathcal{L}_{i}\right)
$$

for $i=1,2$. With these solution expressions, express $\left(\frac{A_{2}}{2 \pi r \sin \theta}-\frac{\tilde{A}_{1}}{2 \pi r \sin \theta}\right) \mathbf{e}_{\varphi}$ as

$$
\frac{f_{2}\left(\mathcal{L}_{2}\right) \sin \left(\mathcal{L}_{2}\right) u_{-, \varphi}\left(f_{2}\left(\mathcal{L}_{2}\right), \mathcal{L}_{2}\right)-f_{1}\left(\tilde{\mathcal{L}}_{1}\right) \sin \left(\tilde{\mathcal{L}}_{1}\right) u_{-, \varphi}\left(f_{1}\left(\tilde{\mathcal{L}}_{1}\right), \tilde{\mathcal{L}}_{1}\right)}{r \sin \theta} \mathbf{e}_{\varphi},
$$




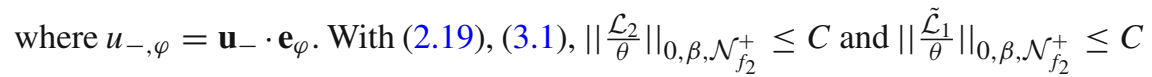
obtained using arguments similar to the ones in the proof of Claim in Lemma 15, $\left\|\mathcal{L}_{2}\right\|_{1, \beta,\left(f_{2}(\theta), r_{1}\right) \times\left(0, \theta_{1}\right)} \leq C,\left\|\tilde{\mathcal{L}}_{1}\right\|_{1, \beta,\left(f_{2}(\theta), r_{1}\right) \times\left(0, \theta_{1}\right)} \leq C$ and Claim, we estimate (3.142) in $C^{\beta}\left(\overline{\mathcal{N}_{f_{2}}^{+}}\right)$. Then we obtain

$$
\begin{aligned}
& \left\|\left(\frac{A_{2}}{2 \pi r \sin \theta}-\frac{\tilde{A}_{1}}{2 \pi r \sin \theta}\right) \mathbf{e}_{\varphi}\right\|_{1,0, \mathcal{N}_{f_{2}}^{+}} \\
& \quad \leq C \sigma\left|f_{2}(0)-f_{1}(0)\right|+C \sigma\left\|\left(\Psi_{2}-\tilde{\Psi}_{1}\right) \mathbf{e}_{\varphi}\right\|_{1, \beta, \mathcal{N}_{f_{2}}^{+}}
\end{aligned}
$$

4. Estimate $\left|f_{2}(0)-f_{1}(0)\right|$.

Substitute $T_{2}-\tilde{T}_{1}=(a)+(b)_{1}+(b)_{2}$ into (3.121). Then we have

$$
\begin{aligned}
& \left.\frac{1}{r_{1} \sin \theta_{1}} \int_{0}^{\theta_{1}} \frac{\rho_{0}^{+}\left((\gamma-1) u_{0}^{+2}+c_{0}^{+2}\right)}{\gamma(\gamma-1) u_{0}^{+} S_{0}^{+}}(b)_{2}\right|_{r=r_{1}} r_{1}^{2} \sin \xi \mathrm{d} \xi \\
& =\frac{1}{r_{1} \sin \theta_{1}} \int_{0}^{\theta_{1}}\left(\mathfrak{f}_{0}\left(T_{2}, p_{e x}\right)-\mathfrak{f}_{0}\left(\tilde{T}_{1}, p_{e x}\right)-\frac{\rho_{0}^{+}\left((\gamma-1) u_{0}^{+2}+c_{0}^{+2}\right)}{\gamma(\gamma-1) u_{0}^{+} S_{0}^{+}}\right. \\
& \left.\left((a)+(b)_{1}\right)+\mathfrak{f}_{1}\left(\Psi_{2} \mathbf{e}_{\varphi}, A_{2}, T_{2}\right)-\mathfrak{f}_{1}\left(\tilde{\Psi}_{1} \mathbf{e}_{\varphi}, \tilde{A}_{1}, \tilde{T}_{1}\right)\right)\left.\right|_{r=r_{1}} r_{1}^{2} \sin \xi \mathrm{d} \xi .
\end{aligned}
$$

Using (3.2) satisfied by ( $\left.f_{i}(0), \Phi_{i} \mathbf{e}_{\varphi}, L_{i}, S_{i}\right)$ for $i=1$, 2, we estimate $\tilde{f}_{1}\left(\Psi_{2} \mathbf{e}_{\varphi}, A_{2}\right.$, $\left.T_{2}\right)-\mathfrak{f}_{1}\left(\tilde{\Psi}_{1} \mathbf{e}_{\varphi}, \tilde{A}_{1}, \tilde{T}_{1}\right)$ in $C^{\beta}\left(\overline{\Gamma_{e x}}\right)$. Then we obtain

$$
\begin{aligned}
& \left\|\mathfrak{f}_{1}\left(\Psi_{2} \mathbf{e}_{\varphi}, A_{2}, T_{2}\right)-\mathfrak{f}_{1}\left(\tilde{\Psi}_{1} \mathbf{e}_{\varphi}, \tilde{A}_{1}, \tilde{T}_{1}\right)\right\|_{0, \beta, \Gamma_{e x}} \leq C \sigma\left(\left\|\left(\Psi_{2}-\tilde{\Psi}_{1}\right) \mathbf{e}_{\varphi}\right\|_{1, \beta, \Gamma_{e x}}\right. \\
& \left.\quad+\left\|\left(\frac{A_{2}}{2 \pi r \sin \theta}-\frac{\tilde{A}_{1}}{2 \pi r \sin \theta}\right) \mathbf{e}_{\varphi}\right\|_{0, \beta, \Gamma_{e x}}+\left\|T_{2}-\tilde{T}_{1}\right\|_{0, \beta, \Gamma_{e x}}\right) .
\end{aligned}
$$

With this estimate, (2.19), (2.20), (3.1) and (3.2) satisfied by $\left(f_{i}(0), \Phi_{i} \mathbf{e}_{\varphi}, L_{i}, S_{i}\right)$ for $i=1,2,(3.137),(3.138),(3.141),(3.143)$ and the fact that $\left(g\left(M_{0}^{-2}\right)\right)^{\prime} S_{i n}$ is strictly positive in $\left[r_{0}, r_{1}\right]$ (see Lemma 2 ), we estimate $f_{2}(0)-f_{1}(0)$ in (3.144). Then we get

$$
\left|f_{2}(0)-f_{1}(0)\right| \leq C_{1} \sigma\left|f_{2}(0)-f_{1}(0)\right|+C \sigma\left\|\left(\Psi_{2}-\tilde{\Psi}_{1}\right) \mathbf{e}_{\varphi}\right\|_{1, \beta, \mathcal{N}_{f_{2}}^{+}}
$$

where $C_{1}$ is a positive constant depending on the data. Take $\sigma_{3}=\min \left(\frac{1}{2 C_{1}}, \sigma_{3}^{(1)}\right)(=$ : $\left.\sigma_{3}^{(2)}\right)$. Then we have

$$
\left|f_{2}(0)-f_{1}(0)\right| \leq C \sigma\left\|\left(\Psi_{2}-\tilde{\Psi}_{1}\right) \mathbf{e}_{\varphi}\right\|_{1, \beta, \mathcal{N}_{f_{2}}^{+}}
$$

5. With the fact that $\Psi_{2} \mathbf{e}_{\varphi}$ and $\tilde{\Psi}_{1} \mathbf{e}_{\varphi}$ are in $C_{\left(-1-\alpha, \Gamma_{w, f_{2}}\right)}^{2, \alpha}\left(\mathcal{N}_{f_{2}}^{+}\right)$, transform (3.119) into an elliptic system form (see (3.35)). Then transform the resultant equation with (3.120) into the following $\mathbf{0}$ boundary value problem 


$$
\begin{aligned}
\operatorname{div}\left(\boldsymbol{A} D\left(\left(\Psi_{2}-\tilde{\Psi}_{1}\right) \mathbf{e}_{\varphi}-\overline{\boldsymbol{h}}\right)\right)-d\left(\left(\Psi_{2}-\tilde{\Psi}_{1}\right) \mathbf{e}_{\varphi}-\overline{\boldsymbol{h}}\right) \\
=\underbrace{-\frac{\rho_{0}^{+\gamma-1}}{(\gamma-1) u_{0}^{+}}\left(1+\frac{\gamma u_{0}^{+2}}{c_{0}^{+2}-u_{0}^{+2}}\right) \frac{\partial_{\theta}\left(T_{2}-\tilde{T}_{1}\right)}{r} \mathbf{e}_{\varphi}-\boldsymbol{F}_{3}}_{\overline{\boldsymbol{F}}} \\
\quad-\operatorname{div}(\boldsymbol{A} D \overline{\boldsymbol{h}})+d \overline{\boldsymbol{h}} \text { in } \mathcal{N}_{f_{2}(0)+f_{s}}^{+} \\
\left(\Psi_{2}-\tilde{\Psi}_{1}\right) \mathbf{e}_{\varphi}-\overline{\boldsymbol{h}}=\mathbf{0} \text { on } \partial \mathcal{N}_{f_{2}(0)+f_{s}}^{+},
\end{aligned}
$$

where $\boldsymbol{A}$ and $d$ are a $(2,2)$ tensor and scalar function defined below (3.41) and

$$
\overline{\boldsymbol{h}}:=\frac{(r-f(\theta)) \frac{r_{1}}{r} \overline{\boldsymbol{h}}_{2}+\left(r_{1}-r\right) \frac{f(\theta)}{r} \overline{\boldsymbol{h}}_{1}}{r_{1}-f(\theta)} .
$$

Here, $\overline{\boldsymbol{F}}$ is of the form

$$
\begin{aligned}
& \sum_{i}\left(A_{2}^{i}-A_{1}^{i}\right) \partial_{r} B_{2}^{i} \mathbf{e}_{\varphi}+\sum_{i} A_{1}^{i} \partial_{r}\left(B_{2}^{i}-B_{1}^{i}\right) \mathbf{e}_{\varphi}+\sum_{i} C_{1}^{i} \partial_{\theta}\left(D_{2}^{i}-D_{1}^{i}\right) \mathbf{e}_{\varphi} \\
& \quad+\left(E_{2}-E_{1}\right) \frac{\partial_{\theta}\left(F_{2} \sin \theta\right)}{\sin \theta} \mathbf{e}_{\varphi}+E_{1} \frac{\partial_{\theta}\left(\left(F_{2}-F_{1}\right) \sin \theta\right)}{\sin \theta} \mathbf{e}_{\varphi}
\end{aligned}
$$

where

$$
\begin{aligned}
& A_{j}^{i} \in C_{\left(-\alpha, \Gamma_{w}^{+}\right)}^{1, \alpha}\left(\mathcal{N}_{f}^{+}\right), B_{j}^{i} \mathbf{e}_{\theta} \in C_{\left(-\alpha, \Gamma_{w}^{+}\right)}^{1, \alpha}\left(\mathcal{N}_{f}^{+}\right), C_{j}^{i} \in C_{\left(-\alpha, \Gamma_{w}^{+}\right)}^{1, \alpha}\left(\mathcal{N}_{f}^{+}\right), \\
& D_{j}^{i} \in C_{\left(-\alpha, \Gamma_{w}^{+}\right)}^{1, \alpha}\left(\mathcal{N}_{f}^{+}\right), E_{j} \mathbf{e}_{\varphi} \in C_{\left(-1-\alpha, \Gamma_{w}^{+}\right)}^{2, \alpha}\left(\mathcal{N}_{f}^{+}\right) \text {and } F_{j} \mathbf{e}_{\varphi} \in C_{\left(-1-\alpha, \Gamma_{w}^{+}\right)}^{2, \alpha}\left(\mathcal{N}_{f}^{+}\right)
\end{aligned}
$$

for $j=1,2$. With arguments similar to the ones in the proof of Lemma 11 using (2.19), (2.20), (3.1), (3.2) satisfied by $\left(f_{i}(0), \Phi_{i} \mathbf{e}_{\varphi}, L_{i}, S_{i}\right)$ for $i=1,2$, (3.145) and

$$
\left\|T_{2}-\tilde{T}_{1}\right\|_{0, \beta, \mathcal{N}_{f_{2}}^{+}} \leq C \sigma\left\|\left(\Psi_{2}-\tilde{\Psi}_{1}\right) \mathbf{e}_{\varphi}\right\|_{1, \beta, \mathcal{N}_{f_{2}}^{+}}
$$

and

$$
\left\|\left(\frac{A_{2}}{2 \pi r \sin \theta}-\frac{\tilde{A}_{1}}{2 \pi r \sin \theta}\right) \mathbf{e}_{\varphi}\right\|_{1,0, \mathcal{N}_{f_{2}}^{+}} \leq C \sigma\left\|\left(\Psi_{2}-\tilde{\Psi}_{1}\right) \mathbf{e}_{\varphi}\right\|_{1, \beta, \mathcal{N}_{f_{2}^{+}}}
$$

obtained from (3.141) and (3.143) using (3.145), we estimate $\left(\Psi_{2}-\tilde{\Psi}_{1}\right) \mathbf{e}_{\varphi}$ in (3.146), (3.147) in $C^{1, \beta}\left(\overline{\mathcal{N}_{f_{2}}^{+}}\right)$(in this argument, we only change $\int_{\mathcal{N}_{f_{2}}^{+}} \sum_{i} A_{1}^{i} \partial_{r}\left(B_{2}^{i}-\right.$ $\left.B_{1}^{i}\right) \mathbf{e}_{\varphi} \boldsymbol{\xi}, \int_{\mathcal{N}_{f_{2}}^{+}} \sum_{i} C_{1}^{i} \partial_{\theta}\left(D_{2}^{i}-D_{1}^{i}\right) \mathbf{e}_{\varphi} \boldsymbol{\xi}$ and $\int_{\mathcal{N}_{f_{2}}^{+}} \operatorname{div}(\boldsymbol{A} D \overline{\boldsymbol{h}}) \boldsymbol{\xi}$ using integration by parts). Then we obtain

$$
\left\|\left(\Psi_{2}-\tilde{\Psi}_{1}\right) \mathbf{e}_{\varphi}\right\|_{1, \beta, \mathcal{N}_{f_{2}}^{+}} \leq C \sigma\left\|\left(\Psi_{2}-\tilde{\Psi}_{1}\right) \mathbf{e}_{\varphi}\right\|_{1, \beta, \mathcal{N}_{f_{2}}^{+}}
$$

Take $\sigma_{3}=\min \left(\frac{1}{2 C}, \sigma_{3}^{(2)}\right)\left(=: \underline{\sigma}_{3}\right)$. Then we have $\Psi_{2} \mathbf{e}_{\varphi}=\tilde{\Psi}_{1} \mathbf{e}_{\varphi}$. Using this fact, we obtain from (3.145), (3.148) and (3.149) $f_{2}(0)=f_{1}(0), T_{2}=\tilde{T}_{1}$ and $A_{2}=\tilde{A}_{1}$. One can see that $\underline{\sigma}_{3}$ depends on the data. This finishes the proof. 


\section{Determination of a Shape of a Shock Location}

In the previous section, for given $\left(\rho_{-}, \mathbf{u}_{-}, p_{-}, p_{e x}, f_{s}^{\prime}\right)$ in a small perturbation of $\left(\rho_{0}^{-}, u_{0}^{-} \mathbf{e}_{r}, p_{0}^{-}, p_{c}, 0\right)$, we found $\left(f(0), \Phi \mathbf{e}_{\varphi}, L, S\right)$ satisfying all the conditions in Problem 2 except (2.47). In this section, to finish the proof of Theorem 1 , for given $\left(\rho_{-}, \mathbf{u}_{-}, p_{-}, p_{e x}\right)$ in a small perturbation of $\left(\rho_{0}^{-}, u_{0}^{-} \mathbf{e}_{r}, p_{0}^{-}, p_{c}\right)$ as in the previous section or in a much small perturbation of $\left(\rho_{0}^{-}, u_{0}^{-} \mathbf{e}_{r}, p_{0}^{-}, p_{c}\right)$ if necessary, we find $f_{s}^{\prime}$ in a small perturbation of 0 as in the previous section such that a solution of Problem 3 for given $\left(\rho_{-}, \mathbf{u}_{-}, p_{-}, p_{e x}, f_{s}^{\prime}\right)=\left(\rho_{0}^{-}, u_{0}^{-} \mathbf{e}_{r}, p_{0}^{-}, p_{c}, f_{s}^{\prime}\right)$ satisfies (2.47).

\subsection{Proof of Theorem 1 (Existence)}

For a constant $\sigma>0$, we define

$$
\begin{aligned}
\mathcal{B}_{\sigma}^{(1)}: & =\left\{\left(\rho_{-}, \mathbf{u}_{-}, p_{-}\right) \in\left(C^{2, \alpha}(\overline{\mathcal{N}})\right)^{3} \mid\right. \\
& \left.\left\|\rho_{-}-\rho_{0}^{-}\right\|_{2, \alpha, \mathcal{N}}+\left\|\mathbf{u}_{-}-u_{0}^{-} \mathbf{e}_{r}\right\|_{2, \alpha, \mathcal{N}}+\left\|p_{-}-p_{0}^{-}\right\|_{2, \alpha, \mathcal{N}} \leq \sigma\right\} \\
\mathcal{B}_{\sigma}^{(2)}: & =\left\{p_{e x} \in C_{\left(-\alpha, \partial \Gamma_{e x}\right)}^{1, \alpha}\left(\Gamma_{e x}\right) \mid\left\|p_{e x}-p_{c}\right\|_{1, \alpha, \Gamma_{e x}}^{\left(-\alpha, \partial \Gamma_{e x}\right)} \leq \sigma\right\}, \\
\mathcal{B}_{\sigma}^{(3)}: & :\left\{h \in C_{\left(-\alpha,\left\{\theta=\theta_{1}\right\}\right), 0}^{1, \alpha}\left(\left(0, \theta_{1}\right)\right) \mid\|h\|_{1, \alpha,\left(0, \theta_{1}\right)}^{\left(-\alpha,\left\{\theta=\theta_{1}\right\}\right)} \leq \sigma\right\}, \\
\mathcal{B}_{\sigma}^{(4)}: & :=\mathcal{B}_{\sigma}^{(1)} \times \mathcal{B}_{\sigma}^{(2)} \times \mathcal{B}_{\sigma}^{(3)} .
\end{aligned}
$$

For given $\left(\rho_{-}, \mathbf{u}_{-}, p_{-}, p_{e x}, f_{s}^{\prime}\right) \in \mathcal{B}_{\sigma}^{(4)}$ for $\sigma \leq \sigma_{3}$, let $\left(f(0), \Phi \mathbf{e}_{\varphi}, L, S\right)$ be a solution of Problem 3 satisfying (3.2) given in Proposition 2. We define

$$
\begin{aligned}
\mathcal{A}\left(\rho_{-}, \mathbf{u}_{-}, p_{-}, p_{e x}, f_{s}^{\prime}\right) & \\
:= & \left.\left(\frac{1}{\varrho\left(\nabla \times\left(\Phi \mathbf{e}_{\varphi}\right), \frac{L}{2 \pi r \sin \theta} \mathbf{e}_{\varphi}, S\right)} \nabla \times\left(\Phi \mathbf{e}_{\varphi}\right) \cdot \boldsymbol{\tau}_{f(0)+f_{s}}-\mathbf{u}_{-} \cdot \boldsymbol{\tau}_{f(0)+f_{s}}\right)\right|_{\Gamma_{f(0)+f_{s}}} \\
& \circ \prod_{r_{s} f(0)+f_{s}}
\end{aligned}
$$

where $\boldsymbol{\tau}_{f(0)+f_{s}}$ is the unit tangent vector on $\Gamma_{f(0)+f_{s}}$ perpendicular to $\mathbf{e}_{\varphi}$ and satisfying $\left(\boldsymbol{\tau}_{f(0)+f_{s}} \times \mathbf{e}_{\varphi}\right) \cdot \boldsymbol{v}_{f(0)+f_{s}}>0$ and $\boldsymbol{v}_{f(0)+f_{s}}$ is the unit normal vector field on $\Gamma_{f(0)+f_{s}}$. Then $\mathcal{A}$ satisfies $\mathcal{A}\left(\rho_{0}^{-}, u_{0}^{-} \mathbf{e}_{r}, p_{0}^{-}, p_{c}, 0\right)=0$ and $\mathcal{A}$ is a map from $\mathcal{B}_{\sigma}^{(4)}$ to $\mathcal{B}_{C \sigma}^{(3)}$ where $C$ is a positive constant depending on the data. If for given $\left(\rho_{-}, \mathbf{u}_{-}, p_{-}, p_{e x}\right) \in \mathcal{B}_{\sigma}^{(1)} \times \mathcal{B}_{\sigma}^{(2)}$ for $\sigma \leq \sigma_{3}$, we find $f_{s}^{\prime} \in \mathcal{B}_{C \sigma}^{(3)}$ for $C \sigma \leq \sigma_{3}$ such that $\mathcal{A}\left(\rho_{-}, \mathbf{u}_{-}, p_{-}, p_{e x}, f_{s}^{\prime}\right)=0$, then $\left(f(0)+f_{s}, \Phi \mathbf{e}_{\varphi}, L, S\right)$ satisfies all the conditions in Problem 2 and thus the existence part of Theorem 1 is proved. We will find such $f_{s}^{\prime}$ using the weak implicit function theorem introduced in [3]. To apply the weak implicit function theorem, we need to prove that $\mathcal{A}$ is continuous, $\mathcal{A}$ is Fréchet differentiable at $\left(\rho_{0}^{-}, u_{0}^{-} \mathbf{e}_{r}, p_{0}^{-}, p_{c}, 0\right)\left(=: \zeta_{0}\right)$ and the partial Fréchet derivative of $\mathcal{A}$ with respect to $f_{s}^{\prime}$ at $\zeta_{0}$ is invertible. We will prove these in the following lemmas.

We first prove that $\mathcal{A}$ is continuous. 
Lemma 16. $\mathcal{A}$ is continuous in $\mathcal{B}_{\sigma_{3}^{*}}^{(4)}$ for a positive constant $\sigma_{3}^{*} \leq \sigma_{3}$ in the sense that if $\zeta^{(k)}:=\left(\rho_{-}^{(k)}, \mathbf{u}_{-}^{(k)}, p_{-}^{(k)}, p_{\text {ex }}^{(k)},\left(f_{S}^{\prime}\right)^{(k)}\right) \in \mathcal{B}_{\sigma_{3}^{*}}^{(4)}$ converges to $\zeta^{(\infty)}:=\left(\rho_{-}^{(\infty)}, \mathbf{u}_{-}^{(\infty)}\right.$, $\left.p_{-}^{(\infty)}, p_{\text {ex }}^{(\infty)},\left(f_{s}^{\prime}\right)^{(\infty)}\right) \in \mathcal{B}_{\sigma_{3}^{*}}^{(4)}$ in $\left(C^{2, \frac{\alpha}{2}}(\overline{\mathcal{N}})\right)^{3} \times C_{\left(-\frac{\alpha}{2}, \partial \Gamma_{e x}\right)}^{1, \frac{\alpha}{2}}\left(\Gamma_{e x}\right) \times C_{\left(-\frac{\alpha}{2},\left\{\theta=\theta_{1}\right\}\right), 0}^{1, \frac{\alpha}{2}}$ $\left(\left(0, \theta_{1}\right)\right)$, then $\mathcal{A}\left(\zeta^{(k)}\right)$ converges to $\mathcal{A}\left(\zeta^{(\infty)}\right)$ in $C_{\left(-\frac{\alpha}{2},\left\{\theta=\theta_{1}\right\}\right), 0}^{1, \frac{\alpha}{2}}\left(\left(0, \theta_{1}\right)\right)$.

Proof. The result is obtained by using the standard argument.

Let $\sigma_{3}^{*}$ be a positive constant $\leq \sigma_{3}$ and to be determined later. Let $\zeta^{(k)}$ for $k=1,2, \ldots$ be a sequence in $\mathcal{B}_{\sigma_{3}^{*}}^{(4)}$ that converges to $\zeta^{(\infty)} \in \mathcal{B}_{\sigma_{3}^{*}}^{(4)}$ in $\left(C^{2, \frac{\alpha}{2}}(\overline{\mathcal{N}})\right)^{3} \times$ $C_{\left(-\frac{\alpha}{2}, \partial \Gamma_{e x}\right)}^{1, \frac{\alpha}{2}}\left(\Gamma_{e x}\right) \times C_{\left(-\frac{\alpha}{2},\left\{\theta=\theta_{1}\right\}\right), 0}^{1, \frac{\alpha}{2}}\left(\left(0, \theta_{1}\right)\right)$. By Proposition 2 , for each given $\left(\rho_{-}\right.$, $\left.\mathbf{u}_{-}, p_{-}, p_{e x}, f_{s}^{\prime}\right)=\zeta^{(k)}$ for $k=1,2, \ldots$ and $\infty$, there exists a unique $\mathcal{U}_{k}:=$ $\left(f(0)^{(k)}, \Phi^{(k)} \mathbf{e}_{\varphi}, L^{(k)}, S^{(k)}\right)$ satisfying (A), (B) and the estimate (3.2) with $\sigma$ replaced by $\sigma_{3}^{*}$. Let $\left(\tilde{\Phi}^{(k)}, \tilde{L}^{(k)}, \tilde{S}^{(k)}\right):=\left(\Phi^{(k)}, L^{(k)}, S^{(k)}\right)\left(\Pi_{r_{s} f(0)^{(k)}+f_{s}^{(k)}}\right)$ for $k=$ $1,2, \ldots$ and $\infty$ and $\tilde{\mathcal{U}}_{k}:=\left(f(0)^{(k)}, \tilde{\Phi}^{(k)} \mathbf{e}_{\varphi}, \tilde{L}^{(k)}, \tilde{S}^{(k)}\right)$ for $k=1,2, \ldots$ and $\infty$ To prove that $\mathcal{A}\left(\zeta^{(k)}\right)$ converges to $\mathcal{A}\left(\zeta^{(\infty)}\right)$ in $C_{\left(-\frac{\alpha}{2},\left\{\theta=\theta_{1}\right\}\right), 0}^{1, \frac{\alpha}{2}}\left(\left(0, \theta_{1}\right)\right)$, we show that $\tilde{\mathcal{U}}_{k}$ converges to $\tilde{\mathcal{U}}_{\infty}$ in $\mathbb{R} \times C_{\left(-1-\frac{\alpha}{2}, \Gamma_{w, r_{s}}^{+}\right)}^{2, \frac{\alpha}{2}}\left(\mathcal{N}_{r_{s}}^{+}\right) \times\left(C_{\left(-\frac{\alpha}{2}, \Gamma_{w, r_{s}}^{+}\right)}^{1, \frac{\alpha}{2}}\left(\mathcal{N}_{r_{s}}^{+}\right)\right)^{2}$.

To do this, we show that if a subsequence of $\tilde{\mathcal{U}}_{k}$ converges in $\mathbb{R} \times C_{\left(-1-\frac{\alpha}{2}, \Gamma_{w, r_{s}}^{+}\right)}^{2, \frac{\alpha}{2}}$ $\left(\mathcal{N}_{r_{s}}^{+}\right) \times\left(C_{\left(-\frac{\alpha}{2}, \Gamma_{w, r_{s}}^{+}\right)}^{1, \frac{\alpha}{2}}\left(\mathcal{N}_{r_{s}}^{+}\right)\right)^{2}$, then it converges to $\tilde{\mathcal{U}}_{\infty}$. Assume that a subsequence $\tilde{\mathcal{U}}_{k_{j}}$ of $\tilde{\mathcal{U}}_{k}$ converges to a function $\tilde{\mathcal{U}}^{*}=\left(f(0)^{*}, \tilde{\Phi}^{*} \mathbf{e}_{\varphi}, \tilde{L}^{*}, \tilde{S}^{*}\right)$ in $\mathbb{R} \times C_{\left(-1-\frac{\alpha}{2}, \Gamma_{w, r_{s}}^{+}\right)}^{2, \frac{\alpha}{2}}$ $\left(\mathcal{N}_{r_{s}}^{+}\right) \times\left(C_{\left(-\frac{\alpha}{2}, \Gamma_{w, r_{s}}^{+}\right)}^{1, \frac{\alpha}{2}}\left(\mathcal{N}_{r_{s}}^{+}\right)\right)^{2}$. Let $(\mathrm{A})^{(k)}$ and $(\mathrm{B})^{(k)}$ be (A) and (B) satisfied by $\mathcal{U}_{k}$, respectively, and let $(\tilde{\mathrm{A}})^{(k)}$ and $(\tilde{\mathrm{B}})^{(k)}$ be $(\mathrm{A})^{(k)}$ and $(\mathrm{B})^{(k)}$ transformed by using $\Pi_{r_{s} f(0)}{ }^{(k)}+f_{s}^{(k)}$, respectively. Since $\mathcal{U}_{k_{j}}$ satisfies $(\mathrm{A})^{\left(k_{j}\right)},(\mathrm{B})^{\left(k_{j}\right)}, \tilde{\mathcal{U}}_{k_{j}}$ satisfies $(\tilde{\mathrm{A}})^{\left(k_{j}\right)},(\tilde{\mathrm{B}})^{\left(k_{j}\right)}$. Take $j \rightarrow \infty$ to $(\tilde{\mathrm{A}})^{\left(k_{j}\right)},(\tilde{\mathrm{B}})^{\left(k_{j}\right)}$. Then since $\zeta^{(k)} \rightarrow \zeta^{(\infty)}$ in $\left(C^{2, \frac{\alpha}{2}}(\overline{\mathcal{N}})\right)^{3} \times C_{\left(-\frac{\alpha}{2}, \partial \Gamma_{e x}\right)}^{1, \frac{\alpha}{2}}\left(\Gamma_{e x}\right) \times C_{\left(-\frac{\alpha}{2},\left\{\theta=\theta_{1}\right\}\right), 0}^{1, \frac{\alpha}{2}}\left(\left(0, \theta_{1}\right)\right)$ and $\tilde{\mathcal{U}}_{k_{j}} \rightarrow \tilde{\mathcal{U}}^{*}$ in $\mathbb{R} \times$ $C_{\left(-1-\frac{\alpha}{2}, \Gamma_{w, r_{s}}^{+}\right)}^{2, \frac{\alpha}{2}}\left(\mathcal{N}_{r_{s}}^{+}\right) \times\left(C_{\left(-\frac{\alpha}{2}, \Gamma_{w, r_{s}}^{+}\right)}^{1, \frac{\alpha}{2}}\left(\mathcal{N}_{r_{s}}^{+}\right)\right)^{2}$, we obtain 1) $(\tilde{\mathrm{A}})^{(\infty)},(\tilde{\mathrm{B}})^{(\infty)}$ with $\tilde{\mathcal{U}}_{\infty}$ replaced by $\tilde{\mathcal{U}}^{*}$. By the facts that $\mathcal{U}_{k}$ for $k=1,2, \ldots$ satisfy (3.2) with $\sigma$ replaced by $\sigma_{3}^{*}$ and $\left(f_{s}^{\prime}\right)^{(k)}$ for $k=1,2, \ldots$ are in $\mathcal{B}_{\sigma_{3}^{*}}^{(3)}$,

$$
\begin{aligned}
& \left.\left|f(0)^{(k)}-r_{s}\right|+\left\|\nabla \times\left(\left(\tilde{\Phi}^{(k)}-\tilde{\Phi}_{0}^{+,(k)}\right) \mathbf{e}_{\varphi}\right)\right\|_{1, \alpha, \mathcal{N}_{r_{s}}^{+}}^{\left(-\alpha, \Gamma_{w}^{+}\right.}\right) \\
& \quad+\left\|\frac{\tilde{L}^{(k)}}{2 \pi \Pi_{r_{s} f(0)^{(k)}+f_{s}^{(k)}}^{r} \sin \theta} \mathbf{e}_{\varphi}\right\|_{1, \alpha, \mathcal{N}_{r_{s}}^{+}}^{\left(-\alpha, \Gamma_{s}^{+}\right)}+\left\|\tilde{S}^{(k)}-\tilde{S}_{0}^{+,(k)}\right\|_{1, \alpha, \mathcal{N}_{r_{s}}^{+}}^{\left(-\alpha, \Gamma_{s}^{+}\right)} \leq C \sigma_{3}^{*},
\end{aligned}
$$

where $\left(\tilde{\Phi}_{0}^{+,(k)}, \tilde{S}_{0}^{+,(k)}\right):=\left(\Phi_{0}^{+}, S_{0}^{+}\right)\left(\Pi_{r_{s} f(0)^{(k)}+f_{s}^{(k)}}\right)$ for $k=1,2, \ldots$ and $C$ is a positive constant depending on the data and independent of $k$. Using the facts that $\left(f_{s}^{\prime}\right)^{(k)} \rightarrow\left(f_{s}^{\prime}\right)^{(\infty)}$ in $C_{\left(-\frac{\alpha}{2},\left\{\theta=\theta_{1}\right\}\right), 0}^{1, \frac{\alpha}{2}}\left(\left(0, \theta_{1}\right)\right)$ and $\tilde{\mathcal{U}}_{k_{j}} \rightarrow \tilde{\mathcal{U}}^{*}$ in $\mathbb{R} \times$ 
$C_{\left(-1-\frac{\alpha}{2}, \Gamma_{w, r_{s}}^{+}\right)}^{2, \frac{\alpha}{2}}\left(\mathcal{N}_{r_{s}}^{+}\right) \times\left(C_{\left(-\frac{\alpha}{2}, \Gamma_{w, r_{s}}^{+}\right)}^{1, \frac{\alpha}{2}}\left(\mathcal{N}_{r_{s}}^{+}\right)\right)^{2}$, we obtain from this inequality

$$
\begin{aligned}
& \left|f(0)^{*}-r_{s}\right|+\left\|\nabla \times\left(\left(\tilde{\Phi}^{*}-\tilde{\Phi}_{0}^{+,(\infty)}\right) \mathbf{e}_{\varphi}\right)\right\|_{1, \alpha, \mathcal{N}_{r_{s}}^{+}}^{\left(-\alpha, \Gamma_{s}^{+}\right)} \\
& \quad+\left\|\frac{\tilde{L}^{*}}{2 \pi \Pi_{r_{s} f(0)^{(\infty)}+f_{s}^{(\infty)}}^{r} \sin \theta} \mathbf{e}_{\varphi}\right\|_{1, \alpha, \mathcal{N}_{r_{s}}^{+}}^{\left(-\alpha, \Gamma_{s}^{+}\right)}+\left\|\tilde{S}^{*}-\tilde{S}_{0}^{+,(\infty)}\right\|_{1, \alpha, \mathcal{N}_{r_{s}}^{+}}^{\left(-\alpha, \Gamma_{s}^{+}\right)} \leq C \sigma_{3}^{*},
\end{aligned}
$$

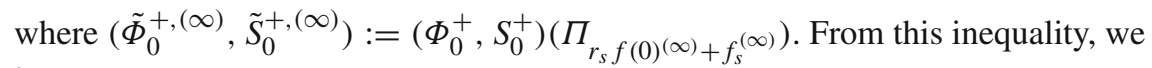
have

$$
\begin{aligned}
& \left|f(0)^{*}-r_{s}\right|+\left\|\nabla \times\left(\left(\Phi^{*}-\Phi_{0}^{+}\right) \mathbf{e}_{\varphi}\right)\right\|_{1, \alpha, \mathcal{N}_{f(0) *+f_{s}}^{+(\infty)}}^{\left(-\alpha, \Gamma^{+}\right.}
\end{aligned}
$$

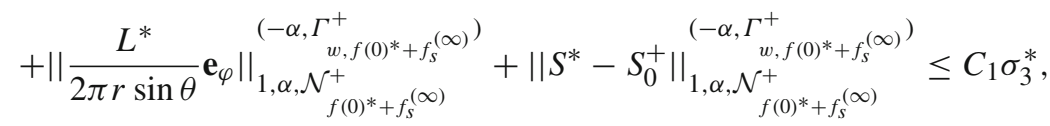

where $\left(\Phi^{*}, L^{*}, S^{*}\right):=\left(\tilde{\Phi}^{*}, \tilde{L}^{*}, \tilde{S}^{*}\right)\left(\Pi_{f(0)^{*}+f_{s}^{(\infty)} r_{s}}\right)$ and $C_{1}$ is a positive constant depending on the data. Take $\sigma_{3}^{*}=\min \left(\frac{C \sigma_{3}}{C_{1}}, \sigma_{3}\right)$ where $C$ is $C$ in (3.2). Then we obtain that 2) $\left(f(0)^{*}, \Phi^{*} \mathbf{e}_{\varphi}, L^{*}, S^{*}\right)$ satisfies (3.2) with $\sigma$ replaced by $\sigma_{3}$. By 1$)$ and 2$),\left(f(0)^{*}, \Phi^{*} \mathbf{e}_{\varphi}, L^{*}, S^{*}\right)$ satisfies (A), (B) for $\left(\rho_{-}, \mathbf{u}_{-}, p_{-}, p_{e x}, f_{s}^{\prime}\right)=\zeta^{(\infty)}$ and (3.2) with $\sigma$ replaced by $\sigma_{3}$. Then since by Proposition $2\left(f(0), \Phi \mathbf{e}_{\varphi}, L, S\right)$ satisfying (A), (B) for $\left(\rho_{-}, \mathbf{u}_{-}, p_{-}, p_{e x}, f_{s}^{\prime}\right)=\zeta^{(\infty)}$ and (3.2) with $\sigma$ replaced by $\sigma_{3}$ is unique, $\left(f(0)^{*}, \Phi^{*} \mathbf{e}_{\varphi}, L^{*}, S^{*}\right)=\left(f(0)^{(\infty)}, \Phi^{(\infty)} \mathbf{e}_{\varphi}, L^{(\infty)}, S^{(\infty)}\right)$. Therefore, $\tilde{\mathcal{U}}^{*}=\tilde{\mathcal{U}}_{\infty}$.

Using the fact that we showed above, we prove that $\tilde{\mathcal{U}}_{k}$ converges to $\tilde{\mathcal{U}}_{\infty}$ in $\mathbb{R} \times C_{\left(-1-\frac{\alpha}{2}, \Gamma_{w, r_{s}}^{+}\right)}^{2, \frac{\alpha}{2}}\left(\mathcal{N}_{r_{s}}^{+}\right) \times\left(C_{\left(-\frac{\alpha}{2}, \Gamma_{w, r_{s}}^{+}\right)}^{1, \frac{\alpha}{2}}\left(\mathcal{N}_{r_{s}}^{+}\right)\right)^{2} . \mathrm{By} \mathbb{R} \times C_{\left(-1-\alpha, \Gamma_{w, r_{s}}^{+}\right)}^{2, \alpha}\left(\mathcal{N}_{r_{s}}^{+}\right) \times$ $\left(C_{\left(-\alpha, \Gamma_{w, r_{s}}^{+}\right)}^{1, \alpha}\left(\mathcal{N}_{r_{s}}^{+}\right)\right)^{2} \Subset \mathbb{R} \times C_{\left(-1-\frac{\alpha}{2}, \Gamma_{w, r_{s}}^{+}\right)}^{2, \frac{\alpha}{2}}\left(\mathcal{N}_{r_{s}}^{+}\right) \times\left(C_{\left(-\frac{\alpha}{2}, \Gamma_{w, r_{s}}^{+}\right)}^{1, \frac{\alpha}{2}}\left(\mathcal{N}_{r_{s}}^{+}\right)\right)^{2}$, every subsequence of $\tilde{\mathcal{U}}_{k}$ has a convergent subsequence in $\mathbb{R} \times C_{\left(-1-\frac{\alpha}{2}, \Gamma_{w, r_{s}}^{+}\right)}^{2, \frac{\alpha}{2}}\left(\mathcal{N}_{r_{s}}^{+}\right) \times$ $\left(C_{\left(-\frac{\alpha}{2}, \Gamma_{w, r_{s}}^{+}\right)}^{1, \frac{\alpha}{2}}\left(\mathcal{N}_{r_{s}}^{+}\right)\right)^{2}$. By the fact that we showed above, this convergent subsequence must converge to $\tilde{\mathcal{U}}_{\infty}$. From this fact, we have $\tilde{\mathcal{U}}_{k} \rightarrow \tilde{\mathcal{U}}_{\infty}$ in $\mathbb{R} \times C_{\left(-1-\frac{\alpha}{2}, \Gamma_{w, r_{s}}\right)}^{2 \frac{\alpha}{2}}$ $\left(\mathcal{N}_{r_{s}}^{+}\right) \times\left(C_{\left(-\frac{\alpha}{2}, \Gamma_{w, r_{s}}^{+}\right)}^{1, \frac{\alpha}{2}}\left(\mathcal{N}_{r_{s}}^{+}\right)\right)^{2}$.

Using this fact, we can conclude that $\mathcal{A}\left(\zeta^{(k)}\right) \rightarrow \mathcal{A}\left(\zeta^{(\infty)}\right)$ in $C_{\left(-\frac{\alpha}{2},\left\{\theta=\theta_{1}\right\}\right), 0}^{1, \frac{\alpha}{2}}$ $\left(\left(0, \theta_{1}\right)\right)$ as $\zeta^{(k)} \in \mathcal{B}_{\sigma_{3}^{*}}^{(4)}$ converges to $\zeta^{(\infty)} \in \mathcal{B}_{\sigma_{3}^{*}}^{(4)}$ in $\left(C^{2, \frac{\alpha}{2}}(\overline{\mathcal{N}})\right)^{3} \times C_{\left(-\frac{\alpha}{2}, \partial \Gamma_{e x}\right)}^{1, \frac{\alpha}{2}}$ $\left(\Gamma_{e x}\right) \times C_{\left(-\frac{\alpha}{2},\left\{\theta=\theta_{1}\right\}\right), 0}^{1, \frac{\alpha}{2}}\left(\left(0, \theta_{1}\right)\right)$. This finishes the proof.

Next, we prove that $\mathcal{A}$ is Fréchet differentiable.

Lemma 17. (i) The mapping $\mathcal{A}$ defined in (4.1) is Fréchet differentiable at $\zeta_{0}:=$ $\left(\rho_{0}^{-}, \mathbf{u}_{0}^{-}, p_{0}^{-}, p_{c}, 0\right)$ as a map from $\left(C_{\left(-\alpha, \Gamma_{w}\right)}^{1, \alpha}(\mathcal{N})\right)^{3} \times C_{\left(-\alpha, \partial \Gamma_{e x}\right)}^{1, \alpha}\left(\Gamma_{e x}\right) \times$ $C_{\left(-\alpha,\left\{\theta=\theta_{1}\right\}\right), 0}^{1, \alpha}\left(\left(0, \theta_{1}\right)\right)$ to $C_{\left(-\alpha,\left\{\theta=\theta_{1}\right\}\right), 0}^{1, \alpha}\left(\left(0, \theta_{1}\right)\right)$. 
(ii) The partial Fréchet derivative of $\mathcal{A}$ with respect to $f_{s}^{\prime}$ at $\zeta_{0}$ is given by

$$
D_{f_{s}^{\prime}} \mathcal{A}\left(\zeta_{0}\right) \tilde{f}_{s}^{\prime}=\frac{1}{\rho_{0}^{+}} \nabla \times\left.\left(\tilde{\Psi}^{\left(\tilde{f}_{s}^{\prime}\right)} \mathbf{e}_{\varphi}\right) \cdot \mathbf{e}_{\theta}\right|_{r=r_{s}}-\left(u_{0}^{-}-u_{0}^{+}\right)\left(r_{s}\right) \frac{\tilde{f}_{s}^{\prime}}{r_{s}}
$$

for $\tilde{f}_{s}^{\prime} \in C_{\left(-\alpha,\left\{\theta=\theta_{1}\right\}\right), 0}^{1, \alpha}\left(\left(0, \theta_{1}\right)\right)$ where $\tilde{\Psi}^{\left(\tilde{f}_{s}^{\prime}\right)} \mathbf{e}_{\varphi}$ is a solution of (4.17), (4.18) for given $\tilde{f}_{s}^{\prime}$.

Proof. In this proof, we prove that $\mathcal{A}$ is Fréchet differentiable as a function of $f_{s}^{\prime}$ at 0 with the other variables fixed at $\left(\rho_{0}^{-}, u_{0}^{-} \mathbf{e}_{r}, p_{0}^{-}, p_{c}\right)$. The Fréchet differentiability of $\mathcal{A}$ as a function of $\left(\rho_{-}, \mathbf{u}_{-}, p_{-}, p_{e x}, f_{s}^{\prime}\right)$ at $\zeta_{0}$ can be proved in a similar way.

In this proof, $C$ s denote positive constants depending on the data. Each $C$ in different inequalities differs from each other.

1. Let $\left(f(0), \Phi \mathbf{e}_{\varphi}, L, S\right)$ be a solution of Problem 3 for $\sigma \in\left(0, \sigma_{3}\right]$ satisfying (3.2).

Let $(\Psi, A, T)=\left(\Phi-\Phi_{0}^{+}, L, S-S_{0}^{+}\right)$and $(\tilde{\Psi}, \tilde{A}, \tilde{T}):=(\Psi, A, T)\left(\Pi_{r_{s} f(0)+f_{s}}\right.$ ). Find the partial Fréchet derivatives of $f(0), \tilde{\Psi} \mathbf{e}_{\varphi}, \tilde{A}$ and $\tilde{T}$ with respect to $f_{s}^{\prime}$ at $\zeta_{0}$.

Let $\tilde{f}_{s}^{\prime}$ be a function in $C_{\left(-\alpha,\left\{\theta=\theta_{1}\right\}\right), 0}^{1, \alpha}\left(\left(0, \theta_{1}\right)\right)$ satisfying $\left\|\tilde{f}_{s}^{\prime}\right\|_{1, \alpha,\left(0, \theta_{1}\right)}^{\left(-\alpha,\left\{\theta=\theta_{1}\right\}\right)}=1$. By Proposition 2 , for each $\left(\rho_{-}, \mathbf{u}_{-}, p_{-}, p_{e x}, f_{s}^{\prime}\right)=\left(\rho_{0}^{-}, u_{0}^{-} \mathbf{e}_{r}, p_{0}^{-}, p_{c}, \varepsilon \tilde{f}_{s}^{\prime}\right)$ for $\varepsilon \in\left[0, \sigma_{3}\right]$, there exists a unique $\left(f_{\varepsilon}(0), \Phi_{\varepsilon} \mathbf{e}_{\varphi}, L_{\varepsilon}, S_{\varepsilon}\right)$ satisfying (A), (B) and the estimate (3.2). Define $\left(\Psi_{\varepsilon}, A_{\varepsilon}, T_{\varepsilon}\right):=\left(\Phi_{\varepsilon}-\Phi_{0}^{+}, L_{\varepsilon}, S_{\varepsilon}-S_{0}^{+}\right)$. Since each $\left(f_{\varepsilon}(0), \Phi_{\varepsilon} \mathbf{e}_{\varphi}, L_{\varepsilon}, S_{\varepsilon}\right)$ satisfies (A), (B) for $\left(\rho_{-}, \mathbf{u}_{-}, p_{-}, p_{e x}, f_{s}^{\prime}\right)=\left(\rho_{0}^{-}, u_{0}^{-} \mathbf{e}_{r}, p_{0}^{-}\right.$, $\left.p_{c}, \varepsilon \tilde{f}_{s}^{\prime}\right)$, each $\left(f_{\varepsilon}(0), \Psi_{\varepsilon} \mathbf{e}_{\varphi}, A_{\varepsilon}, T_{\varepsilon}\right)$ satisfies $\left(\mathrm{A}^{\prime}\right),\left(\mathrm{B}^{\prime}\right)$ for $\left(\rho_{-}, \mathbf{u}_{-}, p_{-}, p_{e x}, f_{s}^{\prime}\right)=$ $\left(\rho_{0}^{-}, u_{0}^{-} \mathbf{e}_{r}, p_{0}^{-}, p_{c}, \varepsilon \tilde{f}_{s}^{\prime}\right)$. Let

$$
\begin{aligned}
& \tilde{f}(0)^{\left(\tilde{f}_{s}^{\prime}\right)}:=\lim _{\varepsilon \rightarrow 0^{+}} \frac{f_{\varepsilon}(0)-f_{0}(0)}{\varepsilon}, \quad \tilde{\Psi}^{\left(\tilde{f}_{s}^{\prime}\right)} \mathbf{e}_{\varphi}=\lim _{\varepsilon \rightarrow 0^{+}} \frac{\tilde{\Psi}_{\varepsilon} \mathbf{e}_{\varphi}-\Psi_{0} \mathbf{e}_{\varphi}}{\varepsilon}, \\
& \tilde{A}^{\left(\tilde{f}_{s}^{\prime}\right)}:=\lim _{\varepsilon \rightarrow 0^{+}} \frac{\tilde{A}_{\varepsilon}-A_{0}}{\varepsilon} \text { and } \tilde{T}^{\left(\tilde{s}_{s}^{\prime}\right)}:=\lim _{\varepsilon \rightarrow 0^{+}} \frac{\tilde{T}_{\varepsilon}-T_{0}}{\varepsilon},
\end{aligned}
$$

where $\tilde{f}(0)^{\left(\tilde{f}_{s}^{\prime}\right)}, \tilde{\Psi}^{\left(\tilde{f}_{s}^{\prime}\right)} \mathbf{e}_{\varphi}, \tilde{A}^{\left(\tilde{f}_{s}^{\prime}\right)}$ and $\tilde{T}^{\left(\tilde{f}_{s}^{\prime}\right)}$ represent the Gâteaux derivatives of $f(0)$, $\tilde{\Psi} \mathbf{e}_{\varphi}, \tilde{A}$ and $\tilde{T}$ in the direction of $\tilde{f}_{s}^{\prime}$ at $\zeta_{0}$. To find the Fréchet derivative of $\mathcal{A}$ with respect to $f_{s}^{\prime}$ at $\zeta_{0}$, we find $\tilde{f}(0)^{\left(\tilde{f}_{s}^{\prime}\right)}, \tilde{\Psi}^{\left(\tilde{f}_{s}^{\prime}\right)} \mathbf{e}_{\varphi}, \tilde{A}^{\left(\tilde{f}_{s}^{\prime}\right)}$ and $\tilde{T}^{\left(\tilde{f}_{s}^{\prime}\right)}$.

Transform $\left(\mathrm{A}^{\prime}\right),\left(\mathrm{B}^{\prime}\right)$ satisfied by $\left(f_{\varepsilon}(0), \Psi_{\varepsilon} \mathbf{e}_{\varphi}, A_{\varepsilon}, T_{\varepsilon}\right)$ into the equations in $\mathcal{N}_{r_{s}}^{+}$or on a part of $\partial \mathcal{N}_{r_{s}}^{+}$by using $\Pi_{r_{s} f_{\varepsilon}}$ where $f_{\varepsilon}:=f_{\varepsilon}(0)+\varepsilon \tilde{f}_{s}$ with $\tilde{f}_{s}=\int_{0}^{\theta} \tilde{f}_{s}^{\prime}$. And then subtract the resultant equations from the same equations satisfied by $\left(f_{0}(0), \Psi_{0} \mathbf{e}_{\varphi}, A_{0}, T_{0}\right)$. Then we obtain

$$
\begin{aligned}
\nabla \times & \left(\frac{1}{\rho_{0}^{+}}\left(1+\frac{u_{0}^{+} \mathbf{e}_{r} \otimes u_{0}^{+} \mathbf{e}_{r}}{c_{0}^{+^{2}}-u_{0}^{+2}}\right) \nabla \times\left(\left(\tilde{\Psi}_{\varepsilon}-\Psi_{0}\right) \mathbf{e}_{\varphi}\right)\right) \\
= & \frac{\rho_{0}^{+\gamma-1}}{(\gamma-1) u_{0}^{+}}\left(1+\frac{\gamma u_{0}^{+2}}{c_{0}^{+2}-u_{0}^{+2}}\right) \frac{\partial_{\theta}\left(\tilde{T}_{\varepsilon}-T_{0}\right)}{r} \mathbf{e}_{\varphi}+F_{0} \\
& \quad+\tilde{\boldsymbol{F}}_{1}\left(\tilde{\Psi}_{\varepsilon} \mathbf{e}_{\varphi}, \tilde{A}_{\varepsilon}, \tilde{T}_{\varepsilon}\right) \text { in } \mathcal{N}_{r_{s}}^{+},
\end{aligned}
$$




$$
\begin{aligned}
& \left(\tilde{\Psi}_{\varepsilon}-\Psi_{0}\right) \mathbf{e}_{\varphi}=\left\{\begin{aligned}
& \mathbf{0} \text { on } \Gamma_{r_{s}}, \Gamma_{w, r_{s}}^{+}, \\
& \frac{1}{r_{1} \sin \theta} \int_{0}^{\theta}\left(-\frac{\rho_{0}^{+}\left((\gamma-1) u_{0}^{+2}+c_{0}^{+2}\right)}{\gamma(\gamma-1) u_{0}^{+} S_{0}^{+}}\left(\tilde{T}_{\varepsilon}-T_{0}\right)\right. \\
&\left.\quad+\mathfrak{f}_{1}\left(\tilde{\Psi}_{\varepsilon} \mathbf{e}_{\varphi}, \tilde{A}_{\varepsilon}, \tilde{T}_{\varepsilon}\right)\right) r_{1}^{2} \sin \xi \mathrm{d} \xi \mathbf{e}_{\varphi} \text { on } \Gamma_{e x},
\end{aligned}\right. \\
& \frac{1}{r_{1} \sin \theta_{1}} \int_{0}^{\theta_{1}}\left(-\frac{\rho_{0}^{+}\left((\gamma-1) u_{0}^{+2}+c_{0}^{+2}\right)}{\gamma(\gamma-1) u_{0}^{+} S_{0}^{+}}\left(\tilde{T}_{\varepsilon}-T_{0}\right)\right. \\
& \left.+\mathfrak{f}_{1}\left(\tilde{\Psi}_{\varepsilon} \mathbf{e}_{\varphi}, \tilde{A}_{\varepsilon}, \tilde{T}_{\varepsilon}\right)\right)\left.\right|_{r=r_{1}} r_{1}^{2} \sin \xi \mathrm{d} \xi \mathbf{e}_{\varphi}=\mathbf{0},
\end{aligned}
$$

$$
\begin{aligned}
& \left\{\begin{array}{c}
\left(M_{\varepsilon}^{T} M_{\varepsilon} \nabla \times\left(\left(\tilde{\Phi}_{0}^{+}+\tilde{\Psi}_{\varepsilon}\right) \mathbf{e}_{\varphi}\right)-\nabla \times\left(\Phi_{0}^{+} \mathbf{e}_{\varphi}\right)\right) \cdot \nabla \tilde{A}_{\varepsilon} \\
\quad+\nabla \times\left(\Phi_{0}^{+} \mathbf{e}_{\varphi}\right) \cdot \nabla\left(\tilde{A}_{\varepsilon}-A_{0}\right)=0 \text { in } \mathcal{N}_{r_{s}}^{+}, \\
\tilde{A}_{\varepsilon}-A_{0}=0 \text { on } \Gamma_{r_{s}}
\end{array}\right. \\
& \left\{\begin{array}{c}
\left(M_{\varepsilon}^{T} M_{\varepsilon} \nabla \times\left(\left(\tilde{\Phi}_{0}^{+}+\tilde{\Psi}_{\varepsilon}\right) \mathbf{e}_{\varphi}\right)-\nabla \times\left(\Phi_{0}^{+} \mathbf{e}_{\varphi}\right)\right) \cdot \nabla \tilde{T}_{\varepsilon} \\
\quad+\nabla \times\left(\Phi_{0}^{+} \mathbf{e}_{\varphi}\right) \cdot \nabla\left(\tilde{T}_{\varepsilon}-T_{0}\right)=0 \text { in } \mathcal{N}_{r_{s}}^{+}, \\
\tilde{T}_{\varepsilon}-T_{0} \\
\quad=\left.g\left(\left(M_{0}^{-} \mathbf{e}_{r} \cdot v_{f_{\varepsilon}}\right)^{2}\right)\right|_{\Gamma_{f_{\varepsilon}}} S_{i n} \circ \Pi_{r_{s} f_{\varepsilon}}-\left(g\left(M_{0}^{-2}\right)\right)\left(r_{s}\right) S_{i n} \text { on } \Gamma_{r_{s}},
\end{array}\right.
\end{aligned}
$$

where

$$
\begin{aligned}
F_{0}= & \nabla \times\left(\frac{1}{\rho_{0}^{+}}\left(1+\frac{u_{0}^{+} \mathbf{e}_{r} \otimes u_{0}^{+} \mathbf{e}_{r}}{c_{0}^{+2}-u_{0}^{+2}}\right) \nabla \times\left(\tilde{\Psi}_{\varepsilon} \mathbf{e}_{\varphi}\right)\right) \\
& -M_{\varepsilon} \nabla \times\left(\left(\frac{1}{\rho_{0}^{+}}\left(1+\frac{u_{0}^{+} \mathbf{e}_{r} \otimes u_{0}^{+} \mathbf{e}_{r}}{c_{0}^{+^{2}}-u_{0}^{+2}}\right)\right)\left(\Pi_{r_{s} f_{\varepsilon}}\right) M_{\varepsilon} \nabla \times\left(\tilde{\Psi}_{\varepsilon} \mathbf{e}_{\varphi}\right)\right) \\
& -\frac{\rho_{0}^{+\gamma-1}}{(\gamma-1) u_{0}^{+}}\left(1+\frac{\gamma u_{0}^{+2}}{c_{0}^{+2}-u_{0}^{+2}}\right) \frac{\partial_{\theta} \tilde{T}_{\varepsilon}}{r} \mathbf{e}_{\varphi} \\
& +\left(\frac{\rho_{0}^{+\gamma-1}}{(\gamma-1) u_{0}^{+}}\left(1+\frac{\gamma u_{0}^{+2}}{c_{0}^{+2}-u_{0}^{+2}}\right) \frac{1}{r}\right)\left(\Pi_{r_{s} f_{\varepsilon}}\right) \\
& \left(\partial_{\tilde{\theta}} \Pi_{f_{\varepsilon} r_{s}}^{r}\left(\Pi_{r_{s} f_{\varepsilon}}\right) \partial_{r} \tilde{T}_{\varepsilon}+\partial_{\theta} \tilde{T}_{\varepsilon}\right) \mathbf{e}_{\varphi},
\end{aligned}
$$

$\left(\tilde{\Psi}_{\varepsilon}, \tilde{A}_{\varepsilon}, \tilde{T}_{\varepsilon}\right):=\left(\Psi_{\varepsilon}, A_{\varepsilon}, T_{\varepsilon}\right) \circ \Pi_{r_{s} f_{\varepsilon}}, \tilde{\Phi}_{0}^{+}=\Phi_{0}^{+} \circ \Pi_{r_{s} f_{\varepsilon}}, M_{\varepsilon}=\left(\frac{\partial \Pi_{f_{\varepsilon} r_{s}}}{\partial \mathrm{y}}\right)\left(\Pi_{r_{s} f_{\varepsilon}}\right)$, $\mathrm{y}$ is the Cartesian coordinate system representing $\mathcal{N}_{f_{\varepsilon}}^{+}, \tilde{\theta}$ is $\theta$ coordinate for $\mathrm{y},(r, \theta)$ is $(r, \theta)$ coordinates for $\mathcal{N}_{r_{s}}^{+}$, and $\Pi_{f_{\varepsilon} r_{s}}^{r}$ is the $r$-component of $\Pi_{f_{\varepsilon} r_{s}}^{*}, \boldsymbol{v}_{f_{\varepsilon}}$ is the unit normal vector field on $\Gamma_{f_{\varepsilon}}$ pointing toward $\mathcal{N}_{f_{\varepsilon}}^{+}$and $\tilde{\boldsymbol{F}}_{1}$ is $\boldsymbol{F}_{1}$ changed by using the transformation $\Pi_{f_{\varepsilon} r_{s}}$. Divide the above equations by $\varepsilon$ and formally take $\varepsilon \rightarrow 0^{+}$ using 


$$
\begin{aligned}
& \left(f_{\varepsilon}(0), \tilde{\Psi}_{\varepsilon}, \tilde{A}_{\varepsilon}, \tilde{T}_{\varepsilon}\right) \rightarrow\left(r_{s}, \Psi_{0}, A_{0}, T_{0}\right)=\left(r_{s}, 0,0,0\right) \text { as } \varepsilon \rightarrow 0^{+}, \\
& \varepsilon \tilde{f}_{s}^{\prime} \rightarrow 0 \text { as } \varepsilon \rightarrow 0^{+}
\end{aligned}
$$

and (4.3). Then we have

$$
\begin{aligned}
& \nabla \times\left(\frac{1}{\rho_{0}^{+}}\left(1+\frac{u_{0}^{+} \mathbf{e}_{r} \otimes u_{0}^{+} \mathbf{e}_{r}}{c_{0}^{+2}-u_{0}^{+2}}\right) \nabla \times\left(\tilde{\Psi}^{\left(\tilde{f}_{s}^{\prime}\right)} \mathbf{e}_{\varphi}\right)\right) \\
& =\frac{\rho_{0}^{+\gamma-1}}{(\gamma-1) u_{0}^{+}}\left(1+\frac{\gamma u_{0}^{+2}}{c_{0}^{+2}-u_{0}^{+2}}\right) \frac{\partial_{\theta} \tilde{T}^{\left(\tilde{f}_{s}^{\prime}\right)}}{r} \mathbf{e}_{\varphi} \text { in } \mathcal{N}_{r_{s}}^{+}, \\
& \tilde{\Psi}^{\left(\tilde{f}_{s}^{\prime}\right)} \mathbf{e}_{\varphi}=\left\{\begin{array}{l}
\mathbf{0} \quad \text { on } \quad \Gamma_{r_{s}}, \Gamma_{w, r_{s}}^{+}, \\
-\frac{1}{r_{1} \sin \theta} \int_{0}^{\theta} \frac{\rho_{0}^{+}\left((\gamma-1) u_{0}^{+2}+c_{0}^{+2}\right)}{\gamma(\gamma-1) u_{0}^{+} S_{0}^{+}} \tilde{T}^{\left(\tilde{f}_{s}^{\prime}\right)} r_{1}^{2} \sin \xi \mathrm{d} \xi \mathbf{e}_{\varphi} \quad \text { on } \Gamma_{e x},
\end{array}\right. \\
& -\left.\frac{1}{r_{1} \sin \theta_{1}} \int_{0}^{\theta_{1}} \frac{\rho_{0}^{+}\left((\gamma-1) u_{0}^{+2}+c_{0}^{+2}\right)}{\gamma(\gamma-1) u_{0}^{+} S_{0}^{+}} \tilde{T}^{\left(\tilde{f}_{s}^{\prime}\right)}\right|_{r=r_{1}} r_{1}^{2} \sin \xi \mathrm{d} \xi \mathbf{e}_{\varphi}=\mathbf{0}, \\
& \left\{\begin{array}{l}
\partial_{r} \tilde{A}^{\left(\tilde{f}_{s}^{\prime}\right)}=0 \text { in } \mathcal{N}_{r_{s}^{+}}^{+}, \\
\tilde{A}^{\left(\tilde{f}_{s}^{\prime}\right)}=0 \text { on } \Gamma_{r_{s}},
\end{array}\right. \\
& \left\{\begin{array}{l}
\partial_{r} \tilde{T}^{\left(\tilde{f}_{s}^{\prime}\right)}=0 \text { in } \mathcal{N}_{r_{s}}^{+}, \\
\tilde{T}^{\left(\tilde{f}_{s}^{\prime}\right)}=\left(g\left(M_{0}^{-2}\right)\right)^{\prime}\left(r_{s}\right) S_{i n}\left(\tilde{f}(0)^{\left(\tilde{f}_{s}^{\prime}\right)}+\tilde{f}_{s}\right) \text { on } \Gamma_{r_{s}} .
\end{array}\right.
\end{aligned}
$$

We solve this system for given $\tilde{f}_{s}^{\prime} \in C_{\left(-\alpha,\left\{\theta=\theta_{1}\right\}\right), 0}^{1, \alpha}\left(\left(0, \theta_{1}\right)\right)$.

By (4.11) and (4.12),

$$
\tilde{A}^{\left(\tilde{f}_{s}^{\prime}\right)}=0 \text { in } \mathcal{N}_{r_{s}}^{+}
$$

and

$$
\tilde{T}^{\left(\tilde{f}_{s}^{\prime}\right)}=\left(g\left(M_{0}^{-2}\right)\right)^{\prime}\left(r_{s}\right) S_{i n}\left(\tilde{f}(0)^{\left(\tilde{f}_{s}^{\prime}\right)}+\tilde{f}_{s}\right) \text { in } \mathcal{N}_{r_{s}}^{+} \text {. }
$$

Substitute (4.14) into (4.10). And then find $\tilde{f}(0)^{\left(\tilde{f}_{s}^{\prime}\right)}$ in the resultant equation. Then we obtain

$$
\tilde{f}(0)^{\left(\tilde{f}_{s}^{\prime}\right)}=\frac{-\int_{0}^{\theta_{1}} \tilde{f}_{s} \sin \zeta d \zeta}{\int_{0}^{\theta_{1}} \sin \zeta d \zeta} .
$$

Substitute this into (4.14) again. Then we have

$$
\tilde{T}^{\left(\tilde{f}_{s}^{\prime}\right)}=\left(g\left(M_{0}^{-2}\right)\right)^{\prime}\left(r_{s}\right) S_{i n}\left(-\frac{\int_{0}^{\theta_{1}} \tilde{f}_{s} \sin \zeta d \zeta}{\int_{0}^{\theta_{1}} \sin \zeta d \zeta}+\tilde{f}_{s}\right) \text { in } \mathcal{N}_{r_{s}}^{+} .
$$

Substituting $\tilde{T}^{\left(\tilde{f}_{s}^{\prime}\right)}$ given in (4.16) into (4.8) and (4.9), we get

$$
\nabla \times\left(\frac{1}{\rho_{0}^{+}}\left(1+\frac{u_{0}^{+} \mathbf{e}_{r} \otimes u_{0}^{+} \mathbf{e}_{r}}{c_{0}^{+2}-u_{0}^{+2}}\right) \nabla \times\left(\tilde{\Psi}^{\left(\tilde{f}_{s}^{\prime}\right)} \mathbf{e}_{\varphi}\right)\right)
$$




$$
\begin{gathered}
=\frac{\rho_{0}^{+\gamma-1}}{(\gamma-1) u_{0}^{+}}\left(1+\frac{\gamma u_{0}^{+2}}{c_{0}^{+2}-u_{0}^{+2}}\right)\left(g\left(M_{0}^{-2}\right)\right)^{\prime}\left(r_{s}\right) S_{i n} \frac{\tilde{f}_{s}^{\prime}}{r} \mathbf{e}_{\varphi} \text { in } \mathcal{N}_{r_{s}}^{+}, \\
\tilde{\Psi}^{\left(\tilde{f}_{s}^{\prime}\right)} \mathbf{e}_{\varphi}=\left\{\begin{array}{r}
\mathbf{0} \text { on } \quad \Gamma_{r_{s}}, \Gamma_{w, r_{s}}^{+}, \\
-\frac{1}{r_{1} \sin \theta} \int_{0}^{\theta} \frac{\rho_{0}^{+}\left((\gamma-1) u_{0}^{+2}+c_{0}^{+2}\right)}{\gamma(\gamma-1) u_{0}^{+} S_{0}^{+}}\left(g\left(M_{0}^{-2}\right)\right)^{\prime}\left(r_{s}\right) S_{i n} \\
\left(-\frac{\int_{0}^{\theta_{1}} \tilde{f}_{s} \sin \zeta d \zeta}{\int_{0}^{\theta_{1}} \sin \zeta d \zeta}+\tilde{f}_{s}\right) r_{1}^{2} \sin \xi \mathrm{d} \xi \mathbf{e}_{\varphi} \text { on } \Gamma_{e x} .
\end{array}\right.
\end{gathered}
$$

Note that since (4.18) is a $C^{0}$ boundary condition, Lemma 7 can be applied to (4.17), (4.18). Using Lemma 7 and the arguments used to prove Lemma 13 (here we can obtain more higher regularity of solutions of (4.17), (4.18) than that of solutions of (3.28), (3.29) in Lemma 7 because $\left.\tilde{f}_{s}^{\prime} \in C_{\left(-\alpha,\left\{\theta=\theta_{1}\right\}\right)}^{1, \alpha}\left(\left(0, \theta_{1}\right)\right)\right)$, we obtain that there exists a unique $C_{\left(-1-\alpha, \Gamma_{w, r_{s}}^{+}\right)}^{3, \alpha}\left(\mathcal{N}_{r_{s}}^{+}\right)$solution $\tilde{\Psi}^{\left(\tilde{f}_{s}^{\prime}\right)} \mathbf{e}_{\varphi}$ of (4.17), (4.18) and this solution satisfies

$$
\left\|\tilde{\Psi}^{\left(\tilde{f}_{s}^{\prime}\right)} \mathbf{e}_{\varphi}\right\|_{3, \alpha, \mathcal{N}_{r_{s}}^{+}}^{\left(-1-\alpha, \Gamma_{w, r_{s}}^{+}\right)} \leq C
$$

for a positive constant $C$ independent of $\tilde{f}_{S}^{\prime}$.

Using (3.2) satisfied by $\left(f_{\varepsilon}(0), \Phi_{\varepsilon} \mathbf{e}_{\varphi}, L_{\varepsilon}, S_{\varepsilon}\right)$, (4.4)-(4.6), (4.8)-(4.10), (4.13), (4.15), (4.16), the solution expressions of $\tilde{A}_{\varepsilon}$ and $\tilde{T}_{\varepsilon}$ obtained by solving $\left(\mathrm{B}^{\prime}\right)$ for $(f(0), \Psi)=\left(f_{\varepsilon}(0), \Psi_{\varepsilon}\right)$ and $\left(\rho_{-}, \mathbf{u}_{-}, p_{-}, p_{e x}, f_{s}^{\prime}\right)=\left(\rho_{0}^{-}, u_{0}^{-} \mathbf{e}_{r}, p_{0}^{-}, p_{c}, \varepsilon \tilde{f}_{s}^{\prime}\right)$ using Lemma 15, and Lemma 7, we can obtain

$$
\begin{aligned}
& \left|f_{\varepsilon}(0)-f_{0}(0)-\varepsilon \tilde{f}(0)^{\left(\tilde{f}_{s}^{\prime}\right)}\right| \leq C \varepsilon^{2}, \\
& \left\|\tilde{\Psi}_{\varepsilon}-\Psi_{0}-\varepsilon \tilde{\Psi}^{\left(\tilde{f}_{s}^{\prime}\right)}\right\|_{2, \alpha, \mathcal{N}_{r_{s}}^{+}}^{\left(-1-\alpha, \Gamma_{w, r_{s}}^{+}\right)} \leq C \varepsilon^{2}, \\
& \left\|\tilde{A}_{\varepsilon}-A_{0}-\varepsilon \tilde{A}^{\left(\tilde{f}_{s}^{\prime}\right)}\right\|_{1, \alpha, \mathcal{N}_{r_{s}}^{+}}^{\left(-\alpha, \Gamma_{s}^{+}\right)}=0, \\
& \left\|\tilde{T}_{\varepsilon}-T_{0}-\varepsilon \tilde{T}^{\left(\tilde{f}_{s}^{\prime}\right)}\right\|_{1, \alpha, \mathcal{N}_{r_{s}}^{+}}^{\left(-\alpha, \Gamma_{s}^{+}\right)} \leq C \varepsilon^{2}
\end{aligned}
$$

for all $\tilde{f}_{s}^{\prime} \in C_{\left(-\alpha,\left\{\theta=\theta_{1}\right\}\right), 0}^{1, \alpha}\left(\left(0, \theta_{1}\right)\right)$ satisfying $\left\|\tilde{f}_{s}^{\prime}\right\|_{1, \alpha,\left(0, \theta_{1}\right)}^{\left(-\alpha,\left\{\theta=\theta_{1}\right\}\right)}=1$ and $\varepsilon \in\left[0, \sigma_{3}\right]$. By (4.13) and (4.15)-(4.18), $\tilde{f}(0)^{\left(\tilde{f}_{s}^{\prime}\right)}, \tilde{\Psi}^{\left(\tilde{f}_{s}^{\prime}\right)} \mathbf{e}_{\varphi}, \tilde{A}^{\left(\tilde{f}_{s}^{\prime}\right)}$ and $\tilde{T}^{\left(\tilde{f}_{s}^{\prime}\right)}$ are bounded linear maps from $C_{\left(-\alpha,\left\{\theta=\theta_{1}\right\}\right), 0}^{1, \alpha}\left(\left(0, \theta_{1}\right)\right)$ to $\mathbb{R}, C_{\left(-1-\alpha, \Gamma_{w, r_{s}}^{+}\right)}^{2, \alpha}\left(\mathcal{N}_{r_{s}}^{+}\right), C_{\left(-\alpha, \Gamma_{w, r_{s}}^{+}\right)}^{1, \alpha}\left(\mathcal{N}_{r_{s}}^{+}\right)$and $C_{\left(-\alpha, \Gamma_{w, r_{s}}^{+}\right)}^{1, \alpha}\left(\mathcal{N}_{r_{s}}^{+}\right)$, respectively. From the above inequalities and this fact, we have that $f(0), \tilde{\Psi} \mathbf{e}_{\varphi}, \tilde{A}$ and $\tilde{T}$ are Fréchet differentiable as a function of $f_{s}^{\prime}$ at 0 with the other variables fixed at $\left(\rho_{0}^{-}, u_{0}^{-} \mathbf{e}_{r}, p_{0}^{-}, p_{c}\right)$ and $\tilde{f}(0)^{\left(\tilde{f}_{s}^{\prime}\right)}, \tilde{\Psi}^{\left(\tilde{f}_{s}^{\prime}\right)} \mathbf{e}_{\varphi}, \tilde{A}^{\left(\tilde{f}_{s}^{\prime}\right)}$ and $\tilde{T}^{\left(\tilde{f}_{s}^{\prime}\right)}$ are the partial Fréchet derivatives of $f(0), \tilde{\Psi} \mathbf{e}_{\varphi}, \tilde{A}$ and $\tilde{T}$ with respect to $f_{s}^{\prime}$ at $\zeta_{0}$, respectively.

2. Show that $\mathcal{A}$ is Fréchet differentiable as a function of $f_{s}^{\prime}$ at 0 with the other variables fixed at $\left(\rho_{0}^{-}, u_{0}^{-} \mathbf{e}_{r}, p_{0}^{-}, p_{c}\right)$. 
By definition, the Gâteaux derivative of $\mathcal{A}$ in the direction $\left(0,0,0,0, \tilde{f}_{s}^{\prime}\right)$ at $\zeta_{0}$ is given by

$$
\begin{aligned}
\lim _{\varepsilon \rightarrow 0^{+}} & \frac{\mathcal{A}\left(\rho_{0}^{-}, u_{0}^{-} \mathbf{e}_{r}, p_{0}^{-}, p_{c}, \varepsilon \tilde{f}_{s}^{\prime}\right)-\mathcal{A}\left(\rho_{0}^{-}, u_{0}^{-} \mathbf{e}_{r}, p_{0}^{-}, p_{c}, 0\right)}{\varepsilon} \\
= & \lim _{\varepsilon \rightarrow 0^{+}} \frac{1}{\varepsilon}\left(\frac{1}{\varrho\left(\nabla \times\left(\left(\Phi_{0}^{+}+\Psi_{\varepsilon}\right) \mathbf{e}_{\varphi}\right), \frac{A_{\varepsilon}}{2 \pi r \sin \theta} \mathbf{e}_{\varphi}, S_{0}^{+}+T_{\varepsilon}\right)}\right. \\
& \nabla \times\left.\left(\left(\Phi_{0}^{+}+\Psi_{\varepsilon}\right) \mathbf{e}_{\varphi}\right) \cdot \boldsymbol{\tau}_{f_{\varepsilon}}\right|_{\Gamma_{f_{\varepsilon}}} \circ \Pi_{r_{s} f_{\varepsilon}}-\left.u_{0}^{-} \mathbf{e}_{r} \cdot \boldsymbol{\tau}_{f_{\varepsilon}}\right|_{\Gamma_{f_{\varepsilon}}} \circ \Pi_{r_{s} f_{\varepsilon}} \\
& \left.-\frac{1}{\varrho\left(\nabla \times\left(\Phi_{0}^{+} \mathbf{e}_{\varphi}\right), 0, S_{0}^{+}\right)} \nabla \times\left.\left(\Phi_{0}^{+} \mathbf{e}_{\varphi}\right) \cdot \mathbf{e}_{\theta}\right|_{\Gamma_{r_{s}}}+\left.u_{0}^{-} \mathbf{e}_{r} \cdot \mathbf{e}_{\theta}\right|_{\Gamma_{r_{s}}}\right)
\end{aligned}
$$

where $\boldsymbol{\tau}_{f_{\varepsilon}}$ is the unit tangent vector on $\Gamma_{f_{\varepsilon}}$ perpendicular to $\mathbf{e}_{\varphi}$ and satisfying $\left(\boldsymbol{\tau}_{f_{\varepsilon}} \times \mathbf{e}_{\varphi}\right) \cdot \boldsymbol{v}_{f_{\varepsilon}}>0$ and $\boldsymbol{v}_{f_{\varepsilon}}$ is the unit normal vector field on $\Gamma_{f_{\varepsilon}}$. As we did in Step 1, formally take $\varepsilon \rightarrow 0^{+}$using (4.3) and (4.7) to the right hand side of the above equation. Then we obtain

$$
\frac{1}{\rho_{0}^{+}} \nabla \times\left.\left(\tilde{\Psi}^{\left(\tilde{f}_{s}^{\prime}\right)} \mathbf{e}_{\varphi}\right) \cdot \mathbf{e}_{\theta}\right|_{r=r_{s}}-\left(u_{0}^{-}-u_{0}^{+}\right)\left(r_{s}\right) \frac{\tilde{f}_{s}^{\prime}}{r_{s}} .
$$

Define a map $L$ by

$$
L \tilde{f}_{s}^{\prime}:=\frac{1}{\rho_{0}^{+}} \nabla \times\left.\left(\tilde{\Psi}^{\left(\tilde{f}_{s}^{\prime}\right)} \mathbf{e}_{\varphi}\right) \cdot \mathbf{e}_{\theta}\right|_{r=r_{s}}-\left(u_{0}^{-}-u_{0}^{+}\right)\left(r_{s}\right) \frac{\tilde{f}_{s}^{\prime}}{r_{s}}
$$

for $\tilde{f}_{s}^{\prime} \in C_{\left(-\alpha,\left\{\theta=\theta_{1}\right\}\right), 0}^{1, \alpha}\left(\left(0, \theta_{1}\right)\right)$ satisfying $\left\|\tilde{f}_{s}^{\prime}\right\|_{1, \alpha,\left(0, \theta_{1}\right)}^{\left(-\alpha,\left\{\theta=\theta_{1}\right\}\right)}=1$ where $\tilde{\Psi}^{\left(\tilde{f}_{s}^{\prime}\right)} \mathbf{e}_{\varphi}$ is a solution of (4.17), (4.18) for given $\tilde{f}_{s}^{\prime} \in C_{\left(-\alpha,\left\{\theta=\theta_{1}\right\}\right), 0}^{1, \alpha}\left(\left(0, \theta_{1}\right)\right)$. Then $L$ is a bounded linear map from $C_{\left(-\alpha,\left\{\theta=\theta_{1}\right\}\right), 0}^{1, \alpha}\left(\left(0, \theta_{1}\right)\right)$ to $C_{\left(-\alpha,\left\{\theta=\theta_{1}\right\}\right), 0}^{1, \alpha}\left(\left(0, \theta_{1}\right)\right)$. Using (3.2) satisfied by $\left(f(0), \Phi_{\varepsilon} \mathbf{e}_{\varphi}, L_{\varepsilon}, S_{\varepsilon}\right)$ and (4.20), one can check that

$$
\left\|\mathcal{A}\left(\rho_{0}^{-}, u_{0}^{-} \mathbf{e}_{r}, p_{0}^{-}, p_{c}, \varepsilon \tilde{f}_{s}^{\prime}\right)-\mathcal{A}\left(\rho_{0}^{-}, u_{0}^{-} \mathbf{e}_{r}, p_{0}^{-}, p_{c}, 0\right)-\varepsilon L \tilde{f}_{s}^{\prime}\right\|_{1, \alpha,\left(0, \theta_{1}\right)}^{\left(-\alpha,\left\{\theta=\theta_{1}\right\}\right)} \leq C \varepsilon^{2}
$$

for any $\tilde{f}_{s}^{\prime} \in C_{\left(-\alpha,\left\{\theta=\theta_{1}\right\}\right), 0}^{1, \alpha}\left(\left(0, \theta_{1}\right)\right)$ satisfying $\left\|\tilde{f}_{s}^{\prime}\right\|_{1, \alpha,\left(0, \theta_{1}\right)}^{\left(-\alpha,\left\{\theta=\theta_{1}\right\}\right)}=1$ and $\varepsilon \in\left[0, \sigma_{3}\right]$. Therefore, $\mathcal{A}$ is Fréchet differentiable as a function of $f_{s}^{\prime}$ at 0 with the other variables fixed at $\left(\rho_{0}^{-}, u_{0}^{-} \mathbf{e}_{r}, p_{0}^{-}, p_{c}\right)$ and $L$ is the partial Fréchet derivative of $\mathcal{A}$ with respect to $f_{s}^{\prime}$ at $\zeta_{0}$. This finishes the proof.

Finally, we prove that the partial Fréchet derivative of $\mathcal{A}$ with respect to $f_{s}^{\prime}$ at $\zeta_{0}$ is invertible. When we prove the invertibility of the partial Fréchet derivative of $\mathcal{A}$ with respect to $f_{s}^{\prime}$ at $\zeta_{0}$, we use eigenfunction expansions of $\tilde{f}_{s}^{\prime}$ and $\tilde{\Psi}^{\left(\tilde{f}_{s}^{\prime}\right)}$. The eigenfunctions used to express $\tilde{f}_{s}^{\prime}$ and $\tilde{\Psi}^{\left(\tilde{f}_{s}^{\prime}\right)}$ are eigenfunctions of the eigenvalue problem

$$
\left\{\begin{array}{l}
\frac{1}{\sin \theta} \partial_{\theta}\left(\sin \theta \partial_{\theta} q\right)-\frac{q}{\sin ^{2} \theta}=-\lambda q \text { in } \theta \in\left(0, \theta_{1}\right) \\
q=0 \text { on } \theta=0, \theta_{1}
\end{array}\right.
$$


which arises from $\theta$-part of the spherical coordinate representation of (4.17), (4.18) (note that this is the associated Legendre equation of type $m=1$ with a general domain that is a singular Sturm-Liouville problem). To express $\tilde{f}_{s}^{\prime}$ and $\tilde{\Psi}^{\left(\tilde{f}_{s}^{\prime}\right)}$ using eigenfunctions of (4.22), we need to prove the orthogonal completeness of the set of eigenfunctions of (4.22). We prove this in the following lemma:

Lemma 18. The eigenvalue problem (4.22) has infinitely countable eigenvalues $\lambda_{j}$ for $j=1,2, \ldots$ satisfying $\lambda_{j} \rightarrow \infty$ as $j \rightarrow \infty$ and $\lambda_{j}>0$. A set of eigenfunctions of (4.22) forms a complete orthorgonal set in $L^{2}\left(\left(0, \theta_{1}\right), \sin \theta d \theta\right)$.

Proof. 1. For given $f \in L^{2}\left(\left(0, \theta_{1}\right), \sin \theta \mathrm{d} \theta\right)$, we consider

$$
\left\{\begin{array}{l}
\frac{1}{\sin \theta} \partial_{\theta}\left(\sin \theta \partial_{\theta} q\right)-\frac{q}{\sin ^{2} \theta}=-f \text { in } \theta \in\left(0, \theta_{1}\right), \\
q=0 \text { on } \theta=0, \theta_{1} .
\end{array}\right.
$$

Write this equation in the form

$$
\int_{0}^{\theta_{1}}\left(\partial_{\theta} q \partial_{\theta} \xi+\frac{q \xi}{\sin ^{2} \theta}\right) \sin \theta d \theta=\int_{0}^{\theta_{1}} f \xi \sin \theta \mathrm{d} \theta
$$

for all $\xi \in H_{0}^{1}\left(\left(0, \theta_{1}\right), \sin \theta \mathrm{d} \theta\right)$. Assume for a moment that there exists a unique $q \in H_{0}^{1}\left(\left(0, \theta_{1}\right), \sin \theta \mathrm{d} \theta\right)$ satisfying (4.24) for all $\xi \in H_{0}^{1}\left(\left(0, \theta_{1}\right), \sin \theta \mathrm{d} \theta\right)$ and this $q$ satisfies

$$
\|q\|_{H_{0}^{1}\left(\left(0, \theta_{1}\right), \sin \theta \mathrm{d} \theta\right)} \leq C\|f\|_{L^{2}\left(\left(0, \theta_{1}\right), \sin \theta \mathrm{d} \theta\right)}
$$

for some positive constant $C$. Using this $q$, we define a map $\mathcal{S}: L^{2}\left(\left(0, \theta_{1}\right)\right.$, $\sin \theta \mathrm{d} \theta) \rightarrow L^{2}\left(\left(0, \theta_{1}\right), \sin \theta \mathrm{d} \theta\right)$ by

$$
\mathcal{S} f=q .
$$

Then $\mathcal{S}$ is a self-adjoint and compact linear operator. Hence, by the spectral theorem for compact self-adjoint operators, $\mathcal{S}$ has contable infinite eigenvalues $\mu_{j}$ satisfying $\mu_{j} \rightarrow 0$ as $j \rightarrow \infty$ and the set of eigenfunctions $q_{j}$ of $\mathcal{S}$ corresponding to $\mu_{j}$ forms a complete orthorgonal set in $L^{2}\left(\left(0, \theta_{1}\right), \sin \theta \mathrm{d} \theta\right)$. From this fact, we obtain that (4.22) has infinitely countable eigenvalues $\lambda_{j} \rightarrow \infty$ as $j \rightarrow \infty$ and the set of eigenfunctions of (4.22) forms a complete orthorgonal set in $L^{2}\left(\left(0, \theta_{1}\right), \sin \theta \mathrm{d} \theta\right)$.

2. Show that there exists a unique $q \in H_{0}^{1}\left(\left(0, \theta_{1}\right), \sin \theta \mathrm{d} \theta\right)$ satisfying (4.24) for all $\xi \in H_{0}^{1}\left(\left(0, \theta_{1}\right), \sin \theta \mathrm{d} \theta\right)$.

For given $f \in L^{2}\left(\left(0, \theta_{1}\right), \sin \theta \mathrm{d} \theta\right)$, we consider

$$
\int_{\mathcal{D}} \delta \boldsymbol{U} \delta \xi=\int_{\mathcal{D}} f(\theta) \mathbf{e}_{\varphi} \boldsymbol{\xi}
$$

for all $\boldsymbol{\xi} \in H_{0}^{1}(\mathcal{D})$ where $\boldsymbol{U}: \mathcal{D} \rightarrow \mathbb{R}^{3}, \boldsymbol{\xi}: \mathcal{D} \rightarrow \mathbb{R}^{3}, \mathcal{D}:=\{(x, y, z) \in$ $\left.S^{2}: \frac{z}{\sqrt{x^{2}+y^{2}+z^{2}}} \geq \cos \theta_{1}\right\}$ and $\delta$ is the tangential gradient operator on $S^{2}$. By the Lax-Milgram theorem, there exists a unique $\boldsymbol{U} \in H_{0}^{1}(\mathcal{D})$ satisfying (4.26) for all $\xi \in H_{0}^{1}(\mathcal{D})$ and this $\boldsymbol{U}$ satisfies

$$
\|\boldsymbol{U}\|_{H_{0}^{1}(\mathcal{D})} \leq C\left\|f \mathbf{e}_{\varphi}\right\|_{L^{2}(\mathcal{D})}
$$


for some positive constant $C$ depending on $\mathcal{D}$. Let $\boldsymbol{U}$ be a function in $H_{0}^{1}(\mathcal{D})$ satisfying (4.26) for all $\xi \in H_{0}^{1}(\mathcal{D})$. Using the standard argument, it can be shown that $\boldsymbol{U}$ satisfies

$$
\|\boldsymbol{U}\|_{H^{2}(\mathcal{D})} \leq C\left\|f \mathbf{e}_{\varphi}\right\|_{L^{2}(\mathcal{D})} .
$$

From this fact, we see that $\boldsymbol{U}$ satisfies

$$
\int_{\mathcal{D}} \Delta_{S^{2}} \boldsymbol{U} \boldsymbol{\xi}=-\int_{\mathcal{D}} f(\theta) \mathbf{e}_{\varphi} \boldsymbol{\xi}
$$

for all $\xi \in H_{0}^{1}(\mathcal{D})$. Using this fact and the fact that the coefficients of $\Delta_{S^{2}} \boldsymbol{U}$ in the spherical coordinate system are independent of $\varphi$, we apply arguments similar to the ones in the proof of Lemma 14 to $\boldsymbol{U}$ (here, we use the facts that a bounded sequence in $H^{2}(\mathcal{D})$ contains a weakly convergent subsequence and a $H_{0}^{1}(\mathcal{D})$ function satisfying (4.26) for all $\boldsymbol{\xi} \in H_{0}^{1}(\mathcal{D})$ is unique). Then we have that $\boldsymbol{U}$ only has the form $u_{\varphi}(\theta) \mathbf{e}_{\varphi}$.

One can see that if $\boldsymbol{U}=u_{\varphi}(\theta) \mathbf{e}_{\varphi} \in H_{0}^{1}(\mathcal{D})$ satisfies (4.26) for all $\boldsymbol{\xi} \in H_{0}^{1}(\mathcal{D})$, then $u_{\varphi} \in H_{0}^{1}\left(\left(0, \theta_{1}\right), \sin \theta \mathrm{d} \theta\right)$ satisfies (4.24) for all $\xi \in H_{0}^{1}\left(\left(0, \theta_{1}\right), \sin \theta \mathrm{d} \theta\right)$ and that if $u_{\varphi} \in H_{0}^{1}\left(\left(0, \theta_{1}\right), \sin \theta \mathrm{d} \theta\right)$ satisfies (4.24) for all $\xi \in H_{0}^{1}\left(\left(0, \theta_{1}\right), \sin \theta \mathrm{d} \theta\right)$, then $\boldsymbol{U}=u_{\varphi}(\theta) \mathbf{e}_{\varphi} \in H_{0}^{1}(\mathcal{D})$ satisfies (4.26) for all $\boldsymbol{\xi} \in H_{0}^{1}(\mathcal{D})$ having the form $\xi(\theta) \mathbf{e}_{\varphi}$. Using this fact, we can deduce that there exists a unique $q \in H_{0}^{1}\left(\left(0, \theta_{1}\right)\right.$, $\sin \theta \mathrm{d} \theta)$ satisfying (4.24) for all $\xi \in H_{0}^{1}\left(\left(0, \theta_{1}\right), \sin \theta \mathrm{d} \theta\right)$. By (4.27), this solution satisfies (4.25).

3. Show that eigenvalues $\lambda_{j}$ of (4.22) are positive.

If $q$ is an eigenfunction of $\mathcal{S}$ corresponding to an eigenvalue $\mu=\frac{1}{\lambda}$, then it holds that

$$
\int_{\mathcal{D}} \delta\left(q \mathbf{e}_{\varphi}\right) \delta \xi=\int_{\mathcal{D}} \lambda q \mathbf{e}_{\varphi} \boldsymbol{\xi}
$$

for all $\xi \in H_{0}^{1}(\mathcal{D})$ having the form $\xi(\theta) \mathbf{e}_{\varphi}$. Using the weak maximum principle, we can have that for $q \mathbf{e}_{\varphi} \in H_{0}^{1}(\mathcal{D})$ to be a nonzero function satisfying (4.28) for all $\xi \in H_{0}^{1}(\mathcal{D})$ having the form $\xi(\theta) \mathbf{e}_{\varphi}, \lambda$ must be positive. Hence, eigenvalues of (4.22) are positive. This finishes the proof.

Then we prove the invertibility of the partial Fréchet derivative of $\mathcal{A}$ with respect to $f_{s}^{\prime}$ at $\zeta_{0}$.

Lemma 19. The partial Fréchet derivative of $\mathcal{A}$ with respect to $f_{s}^{\prime}$ at $\zeta_{0}$ given by (4.2) is an invertible map from $C_{\left(-\alpha,\left\{\theta=\theta_{1}\right\}\right)}^{1, \alpha}\left(\left(0, \theta_{1}\right)\right)$ to $C_{\left(-\alpha,\left\{\theta=\theta_{1}\right\}\right)}^{1, \alpha}\left(\left(0, \theta_{1}\right)\right)$.

Proof. By (4.19) and $C_{\left(-1-\alpha,\left\{\theta=\theta_{1}\right\}\right)}^{2, \alpha}\left(\left(0, \theta_{1}\right)\right) \Subset C_{\left(-\alpha,\left\{\theta=\theta_{1}\right\}\right)}^{1, \alpha}\left(\left(0, \theta_{1}\right)\right), D_{f_{s}^{\prime}} \mathcal{A}\left(\zeta_{0}\right)$ given by (4.2) is of the form $c I-K$ where $c$ is a constant and $K$ is a compact linear map from $C_{\left(-\alpha,\left\{\theta=\theta_{1}\right\}\right), 0}^{1, \alpha}\left(\left(0, \theta_{1}\right)\right)$ to $C_{\left(-\alpha,\left\{\theta=\theta_{1}\right\}\right), 0}^{1, \alpha}\left(\left(0, \theta_{1}\right)\right)$. By the Fredholm alternative, this implies that if $\operatorname{ker} D_{f_{s}^{\prime}} \mathcal{A}\left(\zeta_{0}\right)=\{0\}$, then $D_{f_{s}^{\prime}} \mathcal{A}\left(\zeta_{0}\right)$ is invertible. In this proof, we show that $\operatorname{ker} D_{f_{s}^{\prime}} \mathcal{A}\left(\zeta_{0}\right)=\{0\}$. Since it is obvious that $D_{f_{s}^{\prime}} \mathcal{A}\left(\zeta_{0}\right) 0=$ 0 , we show that $D_{f_{s}^{\prime}} \mathcal{A}\left(\zeta_{0}\right) \tilde{f}_{s}^{\prime}=0$ only if $\tilde{f}_{s}^{\prime}=0$. 
1. Assume that for a nonzero $\tilde{f}_{s}^{\prime} \in C_{\left(-\alpha,\left\{\theta=\theta_{1}\right\}\right), 0}^{1, \alpha}\left(\left(0, \theta_{1}\right)\right), D_{f_{s}^{\prime}} \mathcal{A}\left(\zeta_{0}\right) \tilde{f}_{s}^{\prime}=0$. Then it holds that

$$
\frac{1}{\rho_{0}^{+}} \nabla \times\left.\left(\Psi^{\left(\tilde{f}_{s}^{\prime}\right)} \mathbf{e}_{\varphi}\right) \cdot \mathbf{e}_{\theta}\right|_{r=r_{s}}-\left(u_{0}^{-}-u_{0}^{+}\right)\left(r_{s}\right) \frac{\tilde{f}_{s}^{\prime}}{r_{s}}=0,
$$

where $\tilde{\Psi}^{\left(\tilde{f}_{s}^{\prime}\right)} \mathbf{e}_{\varphi}$ is the $C_{\left(-1-\alpha, \Gamma_{w, r_{s}}^{+}\right)}^{3, \alpha}\left(\mathcal{N}_{r_{s}}^{+}\right)$solution of

$$
\begin{aligned}
& -\frac{1}{\rho_{0}^{+} r^{2}} \partial_{r}\left(r^{2} \partial_{r} \tilde{\Psi}^{\left(\tilde{f}_{s}^{\prime}\right)}\right)+\frac{\partial_{r} \rho_{0}^{+}}{\rho_{0}^{+2} r} \partial_{r}\left(r \tilde{\Psi}^{\left(\tilde{f}_{s}^{\prime}\right)}\right) \\
& -\frac{1}{\rho_{0}^{+} r^{2}}\left(\frac{c_{0}^{+2}}{c_{0}^{+2}-u_{0}^{+2}}\right)\left(\frac{1}{\sin \theta} \partial_{\theta}\left(\sin \theta \partial_{\theta} \tilde{\Psi}^{\left(\tilde{f}_{s}^{\prime}\right)}\right)-\frac{\tilde{\Psi}^{\left(\tilde{f}_{s}^{\prime}\right)}}{\sin ^{2} \theta}\right) \\
& =\frac{\rho_{0}^{+\gamma-1}}{(\gamma-1) u_{0}^{+}}\left(1+\frac{\gamma u_{0}^{+2}}{c_{0}^{+2}-u_{0}^{+2}}\right) \frac{\left(g\left(M_{0}^{-2}\right)\right)^{\prime}\left(r_{s}\right) S_{i n}}{r} \tilde{f}_{s}^{\prime} \text { in } \mathcal{N}_{r_{s}}^{+, *}, \\
& \tilde{\Psi}^{\left(\tilde{f}_{s}^{\prime}\right)}=\left\{\begin{array}{c}
0 \quad \Gamma_{r_{s}}^{*}, \Gamma_{w, r_{s}}^{+*}, \\
-\frac{1}{r_{1} \sin \theta} \int_{0}^{\theta} \frac{\rho_{0}^{+}\left((\gamma-1) u_{0}^{+2}+c_{0}^{+2}\right)}{\gamma(\gamma-1) u_{0}^{+} S_{0}^{+}}\left(g\left(M_{0}^{-2}\right)\right)^{\prime}\left(r_{s}\right) S_{i n} \\
\left(-\frac{\int_{0}^{\theta_{1}} \tilde{f}_{s} \sin \zeta d \zeta}{\int_{0}^{\theta_{1}} \sin \zeta d \zeta}+\tilde{f}_{s}\right) r_{1}^{2} \sin \xi \mathrm{d} \xi \text { on } \Gamma_{e x}^{*},
\end{array}\right.
\end{aligned}
$$

which is the spherical coordinate representation of (4.17), (4.18), for given $\tilde{f}_{s}^{\prime}$. Using $C_{\left(-\alpha,\left\{\theta=\theta_{1}\right\}\right)}^{1, \alpha}\left(\left(0, \theta_{1}\right)\right) \subset L^{2}\left(\left(0, \theta_{1}\right), \sin \theta \mathrm{d} \theta\right)$ and Lemma 18 , we express $\tilde{f}_{s}^{\prime}$ as

$$
\tilde{f}_{s}^{\prime}=\sum_{j=1}^{\infty} c_{j} q_{j}
$$

where $c_{j}$ are constants and $q_{j}$ are eigenfunctions of (4.22) corresponding to eigenvalues $\lambda_{j}$ of (4.22). Define

$$
\tilde{f}_{s, m}^{\prime}:=\sum_{j=1}^{m} c_{j} q_{j}
$$

and $\tilde{f}_{s, m}:=\int_{0}^{\theta} \tilde{f}_{s, m}^{\prime}$. We consider (4.30), (4.31) for given $\tilde{f}_{s}^{\prime}=\tilde{f}_{s, m}^{\prime}$ and $\tilde{f}_{s}=\tilde{f}_{s, m}$.

Using the fact that $q_{j} \in C^{\infty}\left(\left[0, \theta_{1}\right]\right)$ and $\frac{1}{\sin \theta} \partial_{\theta}\left(\sin \theta \partial_{\theta} q\right)-\frac{q}{\sin ^{2} \theta}=\partial_{\theta}$ $\left(\frac{\partial_{\theta}(q \sin \theta)}{\sin \theta}\right)$, we can have

$$
\tilde{f}_{s, m}=\sum_{j=1}^{m}-\frac{c_{j}}{\lambda_{j}}\left(\frac{\partial_{\theta}\left(q_{j} \sin \theta\right)}{\sin \theta}-\left.\frac{\partial_{\theta}\left(q_{j} \sin \theta\right)}{\sin \theta}\right|_{\theta=0}\right) .
$$

Here, $\left.\frac{\partial_{\theta}\left(q_{j} \sin \theta\right)}{\sin \theta}\right|_{\theta=0}$ is bounded because $q_{j}=0$ on $\theta=0$ and $q_{j} \in C^{\infty}\left(\left[0, \theta_{1}\right]\right)$. 
Substitute $\tilde{\Psi}_{m}=\sum_{j=1}^{m} p_{j}(r) q_{j}(\theta), \tilde{f}_{s, m}^{\prime}$ given in (4.33) and $\tilde{f}_{s, m}$ given in (4.34) into the places of $\tilde{\Psi}^{\left(\tilde{f}_{s}^{\prime}\right)}, \tilde{f}_{s}^{\prime}$ and $\tilde{f}_{s}$ in (4.30), (4.31), respectively. Then we obtain

$$
\begin{aligned}
\left(L_{1}:=\right)-\frac{1}{r^{2}}\left(\frac{1}{\rho_{0}^{+}} r^{2} p_{j}^{\prime}\right)^{\prime}+\left(\frac{1}{\rho_{0}^{+} r^{2}}\left(1+\frac{u_{0}^{+2}}{c_{0}^{+2}-u_{0}^{+2}}\right) \lambda_{j}+\frac{\partial_{r} \rho_{0}^{+}}{\rho_{0}^{+2} r}\right) p_{j} \\
=\frac{\rho_{0}^{+\gamma-1}}{(\gamma-1) u_{0}^{+}}\left(1+\frac{\gamma u_{0}^{+2}}{c_{0}^{+2}-u_{0}^{+2}}\right) \frac{\left(g\left(M_{0}^{-}\right)^{2}\right)^{\prime}\left(r_{s}\right) S_{i n}}{r} c_{j} \text { in }\left(r_{s}, r_{1}\right), \\
p_{j}=\left\{\begin{array}{l}
0 \text { on } r=r_{s}, \\
\frac{\rho_{0}^{+}\left((\gamma-1) u_{0}^{+2}+c_{0}^{+2}\right)\left(g\left(M_{0}^{-2}\right)\right)^{\prime}\left(r_{s}\right) S_{i n} r_{1}}{\gamma(\gamma-1) u_{0}^{+} s_{0}^{+} \lambda_{j}} c_{j} \text { on } r=r_{1}
\end{array}\right.
\end{aligned}
$$

for $j=1, \ldots, m$. One can see that $L_{1}$ is of the form $L_{0}-c_{0} I$ where $L_{0}(=$ $\left.\frac{1}{r^{2}}\left(\frac{1}{\rho_{0}^{+}} r^{2} p_{j}^{\prime}\right)^{\prime}\right)$ is an invertible operator and $c_{0}$ is a positive constant. Using the Fredholm alternative and the maximum principle, we can obtain that (4.35), (4.36) for each $j$ has a unique $C^{\infty}\left(\left[r_{s}, r_{1}\right]\right)$ solution. Let $p_{j}$ be the solution of (4.35), (4.36). Then by the fact that $q_{j} \mathbf{e}_{\varphi} \in C^{\infty}(\overline{\mathcal{D}})$ where $\mathcal{D}$ is a domain defined in the proof of Lemma 18, we have that $\tilde{\Psi}_{m} \mathbf{e}_{\varphi}=\sum_{j=1}^{m} p_{j} q_{j} \mathbf{e}_{\varphi}$ is a $C^{\infty}\left(\overline{\mathcal{N}_{r_{s}}^{+}}\right)$solution of (4.17), (4.18) for given $\tilde{f}_{s}^{\prime}=\tilde{f}_{s, m}^{\prime}$ and $\tilde{f}_{s}=\tilde{f}_{s, m}$.

2. Show that there exists a subsequence $\tilde{\Psi}_{m_{l}} \mathbf{e}_{\varphi}$ of $\tilde{\Psi}_{m} \mathbf{e}_{\varphi}$ such that $\tilde{\Psi}_{m_{l}} \mathbf{e}_{\varphi}$ and $D\left(\tilde{\Psi}_{m_{l}} \mathbf{e}_{\varphi}\right)$ weakly converge to $\tilde{\Psi}^{\left(\tilde{f}_{s}^{\prime}\right)} \mathbf{e}_{\varphi}$ and $D\left(\tilde{\Psi}^{\left(\tilde{f}_{s}^{\prime}\right)} \mathbf{e}_{\varphi}\right)$ in $L^{2}\left(\mathcal{N}_{r_{s}}^{+}\right)$, respectively.

Since $\tilde{\Psi}_{m} \mathbf{e}_{\varphi}$ is a $C^{\infty}\left(\overline{\mathcal{N}_{r_{s}}^{+}}\right)$solution of (4.17), (4.18) for given $\tilde{f}_{s}^{\prime}=\tilde{f}_{s, m}^{\prime}$ and $\tilde{f}_{s}=\tilde{f}_{s, m},(4.17),(4.18)$ satisfied by $\tilde{\Psi}_{m} \mathbf{e}_{\varphi}$ can be transformed into the boundary value problem for an elliptic system

$$
\begin{aligned}
\operatorname{div} & \left(\boldsymbol{A} D\left(\tilde{\Psi}_{m} \mathbf{e}_{\varphi}\right)\right)-d\left(\tilde{\Psi}_{m} \mathbf{e}_{\varphi}\right) \\
= & -\frac{\rho_{0}^{+\gamma-1}}{(\gamma-1) u_{0}^{+}}\left(1+\frac{\gamma u_{0}^{+2}}{c_{0}^{+2}-u_{0}^{+2}}\right) \\
& \left(g\left(M_{0}^{-2}\right)\right)^{\prime}\left(r_{s}\right) S_{i n} \frac{\tilde{f}_{s, m}^{\prime}}{r} \mathbf{e}_{\varphi} \text { in } \mathcal{N}_{r_{s}}^{+},
\end{aligned}
$$

where $\boldsymbol{A}$ and $d$ are a $(2,2)$-tensor and scalar function defined below (3.41),

$$
\tilde{\Psi}_{m} \mathbf{e}_{\varphi}=\left\{\begin{aligned}
\mathbf{0} & \text { on } \quad \Gamma_{r_{s}}, \Gamma_{w, r_{s}}^{+}, \\
-\frac{1}{r_{1} \sin \theta} \int_{0}^{\theta} \frac{\rho_{0}^{+}\left((\gamma-1) u_{0}^{+2}+c_{0}^{+2}\right)}{\gamma(\gamma-1) u_{0}^{+} S_{0}^{+}}\left(g\left(M_{0}^{-2}\right)\right)^{\prime}\left(r_{s}\right) S_{i n} & \quad\left(-\frac{\int_{0}^{\theta_{1}} \tilde{f}_{s, m} \sin \zeta d \zeta}{\int_{0}^{\theta_{1}} \sin \zeta d \zeta}+\tilde{f}_{s, m}\right) r_{1}^{2} \sin \xi \mathrm{d} \xi \mathbf{e}_{\varphi}\left(=: h_{m} \mathbf{e}_{\varphi}\right) \text { on } \Gamma_{e x} .
\end{aligned}\right.
$$

Set $\boldsymbol{h}_{m}:=\frac{\left(r-r_{s}\right) r_{1}}{\left(r_{1}-r_{s}\right) r} h_{m} \mathbf{e}_{\varphi}$. Transform (4.37), (4.38) into a $\mathbf{0}$ boundary value problem by substituting $\tilde{\Psi}_{m}^{*} \mathbf{e}_{\varphi}+\boldsymbol{h}_{m}$ into the place of $\tilde{\Psi}_{m} \mathbf{e}_{\varphi}$ in (4.37), (4.38). Write the resultant problem in the following form: 


$$
\begin{aligned}
& \int_{\mathcal{N}_{r_{s}}^{+}} \boldsymbol{A} D\left(\tilde{\Psi}_{m}^{*} \mathbf{e}_{\varphi}\right) D \boldsymbol{\xi}+d \tilde{\Psi}_{m}^{*} \mathbf{e}_{\varphi} \boldsymbol{\xi} \\
& =\int_{\mathcal{N}_{r_{s}}^{+}} \frac{\rho_{0}^{+\gamma-1}}{(\gamma-1) u_{0}^{+}}\left(1+\frac{\gamma u_{0}^{+2}}{c_{0}^{+2}-u_{0}^{+2}}\right)\left(g\left(M_{0}^{-2}\right)\right)^{\prime}\left(r_{s}\right) S_{i n} \frac{\tilde{f}_{s, m}^{\prime}}{r} \mathbf{e}_{\varphi} \boldsymbol{\xi} \\
& \quad-\boldsymbol{A} D \boldsymbol{h}_{m} D \boldsymbol{\xi}-d \boldsymbol{h}_{m} \boldsymbol{\xi}
\end{aligned}
$$

for all $\boldsymbol{\xi} \in H_{0}^{1}\left(\mathcal{N}_{r_{s}}^{+}\right)$where $\tilde{\Psi}_{m}^{*} \mathbf{e}_{\varphi}:=\tilde{\Psi}_{m} \mathbf{e}_{\varphi}-\boldsymbol{h}_{m}$. Using Lemma 9, we obtain from (4.39)

$$
\left\|\tilde{\Psi}_{m}^{*} \mathbf{e}_{\varphi}\right\|_{H_{0}^{1}\left(\mathcal{N}_{r_{s}}^{+}\right)} \leq C\left\|\tilde{f}_{s, m}^{\prime} \mathbf{e}_{\varphi}\right\|_{L^{2}\left(\mathcal{N}_{r_{s}}^{+}\right)}
$$

Since $\left\|\tilde{f}_{s, m}^{\prime} \mathbf{e}_{\varphi}\right\|_{L^{2}\left(\mathcal{N}_{r_{s}}^{+}\right)} \leq\left\|\tilde{f}_{s}^{\prime} \mathbf{e}_{\varphi}\right\|_{L^{2}\left(\mathcal{N}_{r_{s}}^{+}\right)}, \tilde{\Psi}_{m}^{*} \mathbf{e}_{\varphi}$ is a bounded sequence in $H_{0}^{1}$ $\left(\mathcal{N}_{r_{s}}^{+}\right)$. Hence, there exists a subsequence $\tilde{\Psi}_{m_{l}}^{*} \mathbf{e}_{\varphi}$ of $\tilde{\Psi}_{m}^{*} \mathbf{e}_{\varphi}$ and some function $\tilde{\Psi}^{*} \mathbf{e}_{\varphi} \in$ $H_{0}^{1}\left(\mathcal{N}_{r_{s}}^{+}\right)$such that $\tilde{\Psi}_{m_{l}}^{*} \mathbf{e}_{\varphi}$ and $D\left(\tilde{\Psi}_{m_{l}}^{*} \mathbf{e}_{\varphi}\right)$ weakly converge to $\tilde{\Psi}^{*} \mathbf{e}_{\varphi}$ and $D\left(\tilde{\Psi}^{*} \mathbf{e}_{\varphi}\right)$ in $L^{2}\left(\mathcal{N}_{r_{s}}^{+}\right)$, respectively. Take $l \rightarrow \infty$ to (4.39) for $m=m_{l}$. Then by $\tilde{\Psi}_{m_{l}}^{*} \mathbf{e}_{\varphi} \rightarrow$ $\tilde{\Psi}^{*} \mathbf{e}_{\varphi}$ in $L^{2}\left(\left(0, \theta_{1}\right), \sin \theta \mathrm{d} \theta\right), D\left(\tilde{\Psi}_{m_{l}}^{*} \mathbf{e}_{\varphi}\right) \rightarrow D\left(\tilde{\Psi}^{*} \mathbf{e}_{\varphi}\right)$ in $L^{2}\left(\left(0, \theta_{1}\right), \sin \theta \mathrm{d} \theta\right)$ and $\tilde{f}_{s, m}^{\prime} \rightarrow \tilde{f}_{s}^{\prime}$ in $L^{2}\left(\left(0, \theta_{1}\right), \sin \theta \mathrm{d} \theta\right)$, one has (4.39) with $\tilde{f}_{s, m}^{\prime}, \tilde{f}_{s, m}$ and $\tilde{\Psi}_{m}^{*}$ replaced by $\tilde{f}_{s}^{\prime}, \tilde{f}_{s}$ and $\tilde{\Psi}^{*} \mathbf{e}_{\varphi}$, respectively. Thus, $\tilde{\Psi}^{*} \mathbf{e}_{\varphi}$ is a $H_{0}^{1}\left(\mathcal{N}_{r_{s}}^{+}\right)$function satisfying (4.39) with $\tilde{f}_{s, m}^{\prime}$ and $\tilde{f}_{s, m}$ replaced by $\tilde{f}_{s}^{\prime}$ and $\tilde{f}_{s}$, respectively, for all $\xi \in H_{0}^{1}\left(\mathcal{N}_{r_{s}}^{+}\right)$. Let $\boldsymbol{h}$ be $\boldsymbol{h}_{m}$ with $\tilde{f}_{s, m}^{\prime}$ and $\tilde{f}_{s, m}$ replaced by $\tilde{f}_{s}^{\prime}$ and $\tilde{f}_{s}$, respectively. One can see that $\tilde{\Psi}^{\left(\tilde{f}_{s}^{\prime}\right)} \mathbf{e}_{\varphi}-\boldsymbol{h}$ is a $H_{0}^{1}\left(\mathcal{N}_{r_{s}}^{+}\right)$function satisfying (4.39) with $\tilde{f}_{s, m}^{\prime}$ and $\tilde{f}_{s, m}$ replaced by $\tilde{f}_{s}^{\prime}$ and $\tilde{f}_{s}$, respectively, for all $\xi \in H_{0}^{1}\left(\mathcal{N}_{r_{s}}^{+}\right)$. By Lemma 9 , a $H_{0}^{1}\left(\mathcal{N}_{r_{s}}^{+}\right)$function satisfying (4.39) with $\tilde{f}_{s, m}^{\prime}$ and $\tilde{f}_{s, m}$ replaced by $\tilde{f}_{s}^{\prime}$ and $\tilde{f}_{s}$, respectively, for all $\boldsymbol{\xi} \in H_{0}^{1}\left(\mathcal{N}_{r_{s}}^{+}\right)$is unique. Hence, $\tilde{\Psi}^{*} \mathbf{e}_{\varphi}=\tilde{\Psi}^{\left(\tilde{f}_{s}^{\prime}\right)} \mathbf{e}_{\varphi}-\boldsymbol{h}$. From this, we can conclude that $\tilde{\Psi}_{m_{l}} \mathbf{e}_{\varphi}$ and $D\left(\tilde{\Psi}_{m_{l}} \mathbf{e}_{\varphi}\right)$ weakly converge to $\tilde{\Psi}^{\left(\tilde{f}_{s}^{\prime}\right)} \mathbf{e}_{\varphi}$ and $D\left(\tilde{\Psi}^{\left(\tilde{f}_{s}^{\prime}\right)} \mathbf{e}_{\varphi}\right)$ in $L^{2}\left(\mathcal{N}_{r_{s}}^{+}\right)$, respectively.

3. Show that $D_{f_{s}^{\prime}} \mathcal{A}\left(\zeta_{0}\right) \tilde{f}_{s}^{\prime}=0$ only if $\tilde{f}_{s}^{\prime}=0$.

Since $\tilde{f}_{s}^{\prime} \neq 0$ by the assumption, there exists $k \in \mathbb{N}$ such that $c_{k}>0$ or $c_{k}<0$ in the expression of $\tilde{f}_{s}^{\prime}$ in (4.32). Without loss of generality, assume that $c_{k}>0$ for some $k \in \mathbb{N}$. Then since $\lambda_{k}>0$ by Lemma 18, $p_{k}$, that is, the solution of (4.35), (4.36) for $j=k$, satisfies $p_{k}\left(r_{1}\right)>0$. Using this fact, $p_{k}\left(r_{s}\right)=0$ and the form of (4.35), we can deduce that $p_{k} \geq 0$ in $\left[r_{s}, r_{1}\right]$. Thus, $p_{k}^{\prime}\left(r_{s}\right) \geq 0$.

Write (4.29) in the form

$$
\int_{0}^{\theta_{1}}\left(-\left.\frac{1}{\rho_{0}^{+}} \frac{1}{r} \partial_{r}\left(r \tilde{\Psi}^{\left(\tilde{f}_{s}^{\prime}\right)}\right)\right|_{r=r_{s}}-\left(u_{0}^{-}-u_{0}^{+}\right)\left(r_{s}\right) \frac{\tilde{f}_{s}^{\prime}}{r_{s}}\right) \xi \sin \theta d \theta=0
$$

for all $\xi \in L^{2}\left(\left(0, \theta_{1}\right), \sin \theta \mathrm{d} \theta\right)$. 
Rewrite this as

$$
\begin{aligned}
& \int_{0}^{\theta_{1}}\left(-\frac{1}{\rho_{0}^{+}} \sum_{j=1}^{m_{l}} \frac{\partial_{r}\left(r p_{j}\right)}{r} q_{j}-\left(u_{0}^{-}-u_{0}^{+}\right)\left(r_{s}\right) \sum_{j=1}^{m_{l}} \frac{c_{j} q_{j}}{r_{s}}\right. \\
& \quad-\left.\frac{1}{\rho_{0}^{+}} \frac{1}{r} \partial_{r}\left(r \tilde{\Psi}^{\left(\tilde{f}_{s}^{\prime}\right)}\right)\right|_{r=r_{s}}-\left(u_{0}^{-}-u_{0}^{+}\right)\left(r_{s}\right) \frac{\tilde{f}_{s}^{\prime}}{r_{s}} \\
& \left.\quad-\left(-\frac{1}{\rho_{0}^{+}} \sum_{j=1}^{m_{l}} \frac{\partial_{r}\left(r p_{j}\right)}{r} q_{j}-\left(u_{0}^{-}-u_{0}^{+}\right)\left(r_{s}\right) \sum_{j=1}^{m_{l}} \frac{c_{j} q_{j}}{r_{s}}\right)\right) \xi \sin \theta d \theta=0
\end{aligned}
$$

for all $\xi \in L^{2}\left(\left(0, \theta_{1}\right), \sin \theta \mathrm{d} \theta\right)$. Since $p_{k}^{\prime}\left(r_{s}\right) \geq 0, p_{k}\left(r_{s}\right)=0,\left(u_{0}^{-}-u_{0}^{+}\right)\left(r_{s}\right)>0$, $\tilde{\Psi}_{m_{l}} \mathbf{e}_{\varphi} \rightarrow \tilde{\Psi}^{\left(\tilde{f}_{s}^{\prime}\right)} \mathbf{e}_{\varphi}$ in $L^{2}\left(\mathcal{N}_{r_{s}}^{+}\right)$and $D\left(\tilde{\Psi}_{m_{l}} \mathbf{e}_{\varphi}\right) \rightarrow D\left(\tilde{\Psi}^{\left(\tilde{f}_{s}^{\prime}\right)} \mathbf{e}_{\varphi}\right)$ in $L^{2}\left(\mathcal{N}_{r_{s}}^{+}\right)$, for a sufficiently large $l$ such that $m_{l} \geq k$, if we take $\xi=q_{k}$, then the left hand side of (4.40) becomes a negative number (here we used the trace theorem). This contradicts to the assumption that $D_{f_{s}^{\prime}} \mathcal{A}\left(\zeta_{0}\right) \tilde{f}_{s}^{\prime}=0$. This finishes our proof.

Applying the weak implicit function theorem introduced in [3] with the results in Lemmas 16, 17 and 19, we obtain the result of the existence part of Theorem 1.

\subsection{Proof of Theorem 1 (Uniqueness)}

Finally, we prove the uniqueness part of Theorem 1.

Proof of Theorem 1 (Uniqueness) Let $\bar{\sigma}_{1}$ be a positive constant $\leq \sigma_{3}$ obtained in the previous subsection such that if $\sigma \leq \bar{\sigma}_{1}$, then Problem 2 has a solution satisfying

$$
\begin{aligned}
& \left|f(0)-r_{s}\right|+\left\|f_{s}^{\prime}\right\|_{1, \alpha,\left(0, \theta_{1}\right)}^{\left(-\alpha,\left\{\theta=\theta_{1}\right\}\right)}+\left\|\nabla \times\left(\left(\Phi-\Phi_{0}^{+}\right) \mathbf{e}_{\varphi}\right)\right\|_{1, \alpha, \mathcal{N}_{f}^{+}}^{\left(-\alpha, \Gamma_{w}^{+}\right)} \\
& \quad+\left\|\frac{L}{2 \pi r \sin \theta} \mathbf{e}_{\varphi}\right\|_{1, \alpha, \mathcal{N}_{f}^{+}}^{\left(-\alpha, \Gamma_{w}^{+}\right)}+\left\|S-S_{0}^{+}\right\|_{1, \alpha, \mathcal{N}_{f}^{+}}^{\left(-\alpha, \Gamma_{w}^{+}\right)} \leq C \sigma
\end{aligned}
$$

where $C$ is a positive constant depending on the data. Let $\sigma_{1}$ be a positive constant $\leq \bar{\sigma}_{1}$ and to be determined later. Suppose that there exist two solutions $\left(f_{i}, \Phi_{i} \mathbf{e}_{\varphi}, L_{i}, S_{i}\right)$ for $i=1,2$ of Problem 2 for $\sigma \leq \sigma_{1}$ satisfing (4.41).

We will prove that there exists a positive constant $\underline{\sigma_{1}} \leq \bar{\sigma}_{1}$ such that if $\sigma_{1}=\underline{\sigma}_{1}$, then

$$
\left(f_{1}, \Phi_{1} \mathbf{e}_{\varphi}, L_{1}, S_{1}\right)=\left(f_{2}, \Phi_{2} \mathbf{e}_{\varphi}, L_{2}, S_{2}\right) .
$$

In this proof, $C$ s and $C_{i}$ for $i=1,2, \ldots$ denote positive constants depending on the data. Each $C$ in different inequalities differs from each other. In this proof, when we estimate quantities, we will use all or a part of the conditions $\left(\rho_{-}, \mathbf{u}_{-}, p_{-}\right) \in \mathcal{B}_{\sigma}^{(1)}$, $p_{e x} \in \mathcal{B}_{\sigma}^{(3)},(4.41)$ satisfied by $\left(f_{i}, \Phi_{i} \mathbf{e}_{\varphi}, L_{i}, S_{i}\right)$ for $i=1,2$ for $\sigma \leq \sigma_{3}$ without mentioning that we use these conditions.

Case 1. $f_{s, 1}=f_{s, 2}$ where $f_{s, i}:=f_{i}-f_{i}(0)$ for $i=1,2$. 
By (4.41) satisfied by $\left(f_{i}, \Phi_{i} \mathbf{e}_{\varphi}, L_{i}, S_{i}\right)$,

$$
\left\|f_{s, i}^{\prime}\right\|_{1, \alpha,\left(0, \theta_{1}\right)}^{\left(-\alpha,\left\{\theta=\theta_{1}\right\}\right)} \leq C_{1} \sigma
$$

for $i=1,2$. Choose $\sigma_{1}=\min \left(\bar{\sigma}_{1}, \frac{C_{3} \sigma_{3}}{C_{2}}, \frac{\sigma_{3}}{C_{1}}\right)\left(=: \sigma_{1}^{(1)}\right)$ where $C_{2}$ and $C_{3}$ are $C$ in (4.41) and (3.2), respectively. Then $\left(\rho_{-}, \mathbf{u}_{-}, p_{-}, p_{e x}, f_{s}^{\prime}\right) \in \mathcal{B}_{\sigma}^{(4)}$ for $\sigma \in\left(0, \sigma_{3}\right]$ and $\left(f_{i}(0), \Phi_{i} \mathbf{e}_{\varphi}, L_{i}, S_{i}\right)$ satisfies (3.2) for $f_{s}=f_{s, i}$, so the hypothesis in Proposition 2 is satisfied. If $f_{s, 1}=f_{s, 2}$, then by Proposition $2,\left(f_{1}(0), \Phi_{1} \mathbf{e}_{\varphi}, L_{1}, S_{1}\right)=$ $\left(f_{2}(0), \Phi_{2} \mathbf{e}_{\varphi}, L_{2}, S_{2}\right)$. From this, we have (4.42).

Case 2. General case.

1. Let $\left(\Psi_{i}, A_{i}, T_{i}\right):=\left(\Phi_{i}-\Phi_{0}^{+}, L_{i}, S_{i}-S_{0}^{+}\right)$and $\left(\tilde{\Psi}_{i}, \tilde{A}_{i}, \tilde{T}_{i}\right):=\left(\frac{\tilde{W}_{i}}{2 \pi r \sin \theta}\right.$, $\left.A_{i}\left(\Pi_{r_{s} f_{i}}\right), T_{i}\left(\Pi_{r_{s}} f_{i}\right)\right)$ for $i=1,2$ with $\tilde{W}_{i}:=W_{i}\left(\Pi_{r_{s}} f_{i}\right)$ and $W_{i}:=2 \pi r \sin \theta \Psi_{i}$. Show that

$$
\begin{aligned}
& \left|f_{2}(0)-f_{1}(0)\right|+\left\|\left(\tilde{\Psi}_{2}-\tilde{\Psi}_{1}\right) \mathbf{e}_{\varphi}\right\|_{1, \beta, \mathcal{N}_{r_{s}}^{+}} \\
& \quad+\left\|\frac{\tilde{A}_{2}}{2 \pi r \sin \theta}-\frac{\tilde{A}_{1}}{2 \pi r \sin \theta}\right\|_{0, \beta, \mathcal{N}_{r_{s}}^{+}}+\left\|\tilde{T}_{2}-\tilde{T}_{1}\right\|_{0, \beta, \mathcal{N}_{r_{s}}^{+}} \\
& \quad \leq C\left\|f_{s, 2}^{\prime}-f_{s, 1}^{\prime}\right\|_{0, \beta,\left(0, \theta_{1}\right)},
\end{aligned}
$$

where $\beta$ is a positive constant given in the proof of the uniqueness part of Proposition 2 .

By the assumption, $\left(f(0), f_{s}, \Psi \mathbf{e}_{\varphi}, L, T\right)=\left(f_{i}(0), f_{s, i}, \Psi_{i} \mathbf{e}_{\varphi}, A_{i}, T_{i}\right)$ for $i=$ 1,2 satisfy $\left(\mathrm{A}^{\prime}\right)$. Transform $\left(\mathrm{A}^{\prime}\right)$ satisfied by $\left(f_{i}(0), f_{s, i}, \Psi_{i} \mathbf{e}_{\varphi}, A_{i}, T_{i}\right)$ for $i=1,2$ into equations in $\mathcal{N}_{r_{s}}^{+}$or on a part of $\partial \mathcal{N}_{r_{s}}^{+}$by using $\Pi_{r_{s} f_{i}}$ in the way that we transformed ( $\left.\mathrm{A}^{\prime}\right)$ satisfied by $\left(f_{1}(0), \Psi_{1} \mathbf{e}_{\varphi}, A_{1}, T_{1}\right)$ in the proof of the uniqueness part of Proposition 2. Then we obtain

$$
\begin{aligned}
& M_{i} \nabla \times\left(\frac{1}{\rho_{0}^{+}}\left(1+\frac{u_{0}^{+} \mathbf{e}_{r} \otimes u_{0}^{+} \mathbf{e}_{r}}{c_{0}^{+2}-u_{0}^{+2}}\right)\left(\Pi_{r_{s} f_{i}}\right) N_{i} \nabla \times\left(\tilde{\Psi}_{i} \mathbf{e}_{\varphi}\right)\right) \\
& =\left(\frac{\rho_{0}^{+\gamma-1}}{(\gamma-1) u_{0}^{+}}\left(1+\frac{\gamma u_{0}^{+2}}{c_{0}^{+2}-u_{0}^{+2}}\right)\right)\left(\Pi_{r_{s} f_{i}}\right) \frac{\left(\partial_{\tilde{\theta}_{i}} \Pi_{f_{i} r_{s}}^{*, r}\right)\left(\Pi_{r_{s} f_{i}}\right) \partial_{r} \tilde{T}_{i}+\partial_{\theta} \tilde{T}_{i}}{\Pi_{r_{s} f_{i}}^{*, r}} \mathbf{e}_{\varphi} \\
& +\tilde{\boldsymbol{F}}_{1 i}\left(\tilde{\Psi}_{i} \mathbf{e}_{\varphi}, \tilde{A}_{i}, \tilde{T}_{i}\right) \text { in } \mathcal{N}_{r_{s}}^{+}, \\
& \tilde{\Psi}_{i} \mathbf{e}_{\varphi}=\left\{\begin{array}{l}
\frac{\Pi_{r_{s} f_{i}}^{*, r}\left(\Phi_{-}-\Phi_{0}^{-}\right)\left(\Pi_{r_{s} f_{i}}\right)}{r} \mathbf{e}_{\varphi} \text { on } \Gamma_{r_{s}}, \\
\frac{r_{0}\left(\Phi_{-}-\Phi_{0}^{-}\right)\left(r_{0}, \theta_{1}\right)}{r} \mathbf{e}_{\varphi} \text { on } \Gamma_{w, r_{s}}^{+}:=\Gamma_{w} \cap\left\{r>r_{s}\right\}, \\
\left(\frac { 1 } { r \operatorname { s i n } \theta } \int _ { 0 } ^ { \theta } \left(\mathfrak{f}_{0}\left(\tilde{T}_{i}, p_{e x}\right)-\frac{\rho_{0}^{+}\left((\gamma-1) u_{0}^{+2}+c_{0}^{+2}\right)}{\gamma(\gamma-1) u_{0}^{+} S_{0}^{+}} \tilde{T}_{i}\right.\right. \\
\left.\left.\quad+\mathfrak{f}_{1}\left(\tilde{\Psi}_{i} \mathbf{e}_{\varphi}, \tilde{A}_{i}, \tilde{T}_{i}\right)\right) r^{2} \sin \xi \mathrm{d} \xi\right) \mathbf{e}_{\varphi} \text { on } \Gamma_{e x},
\end{array}\right. \\
& \frac{1}{r \sin \theta} \int_{0}^{\theta}\left(\mathfrak{f}_{0}\left(\tilde{T}_{i}, p_{e x}\right)-\frac{\rho_{0}^{+}\left((\gamma-1) u_{0}^{+2}+c_{0}^{+2}\right)}{\gamma(\gamma-1) u_{0}^{+} S_{0}^{+}} \tilde{T}_{i}\right. \\
& \left.+\mathfrak{f}_{1}\left(\tilde{\Psi}_{i} \mathbf{e}_{\varphi}, \tilde{A}_{i}, \tilde{T}_{i}\right)\right) r^{2} \sin \xi \mathrm{d} \xi=\frac{r_{0}\left(\Phi_{-}-\Phi_{0}^{-}\right)\left(r_{0}, \theta_{1}\right)}{r_{1}}
\end{aligned}
$$


for $i=1,2$ where $M_{i}=\left(\frac{\partial \Pi_{f_{i} r_{s}}}{\partial \mathrm{y}_{i}}\right)\left(\Pi_{r_{s} f_{i}}\right)$ for $i=1,2, N_{i}=\frac{r^{2}}{\left(\Pi_{r_{s} f_{i}}\right)^{2}} \mathbf{e}_{r} \otimes \mathbf{e}_{r}-$ $\frac{\left(\partial_{\tilde{\theta}_{i}} \Pi_{f_{i} s_{s}}^{*, r}\right)\left(\Pi_{r_{s} f_{i}}\right)}{\left(\Pi_{r_{s} f_{i}}^{*, r}\right)^{2}} r \mathbf{e}_{r} \otimes \mathbf{e}_{\theta}+\frac{\left(\partial_{\tilde{r}_{i}} \Pi_{f_{i} r_{s}}^{*, r}\right)\left(\Pi_{r_{s} f_{i}}\right)}{\Pi_{r_{s} f_{i}}^{*, r}} r \mathbf{e}_{\theta} \otimes \mathbf{e}_{\theta}, \mathrm{y} i$ for $i=1,2$ are the Cartesian coordinate systems representing $\mathcal{N}_{f_{i}}^{+}$, respectively, $\left(\tilde{r}_{i}, \tilde{\theta}_{i}\right)$ are $(r, \theta)$ coordinates for $\mathrm{y}_{i}$, respectively, $(r, \theta)=\Pi_{f_{i} r_{s}}^{*}\left(\tilde{r}_{i}, \tilde{\theta}_{i}\right), \Pi_{r_{s} f_{i}}^{*, r}$ and $\Pi_{f_{i} r_{s}}^{*, r}$ are the $r$-components of $\Pi_{r_{s} f_{i}}^{*}$ and $\Pi_{f_{i} r_{s}}^{*}$, respectively, and $\tilde{\boldsymbol{F}}_{1 i}$ for $i=1,2$ are $\boldsymbol{F}_{1}$ changed by using the transformation $\Pi_{r_{s} f_{i}}$ for $i=1,2$ using the relations $\left(\nabla \times\left(\Psi_{i} \mathbf{e}_{\varphi}\right)\right)\left(\Pi_{r_{s} f_{i}}\right)=$ $N_{i} \nabla \times\left(\tilde{\Psi}_{i} \mathbf{e}_{\varphi}\right)$ and $\left(\nabla \times\left(\frac{A}{2 \pi r \sin \theta} \mathbf{e}_{\varphi}\right)\right)\left(\Pi_{r_{s} f_{i}}\right)=N_{i} \nabla \times\left(\frac{\tilde{A}_{i}}{2 \pi r \sin \theta} \mathbf{e}_{\varphi}\right)$. Subtract (4.44)-(4.46) for $i=1$ from the same equations for $i=2$. Then we have

$$
\begin{aligned}
& \nabla \times\left(\frac{1}{\rho_{0}^{+}}\left(1+\frac{u_{0}^{+} \mathbf{e}_{r} \otimes u_{0}^{+} \mathbf{e}_{r}}{c_{0}^{+2}-u_{0}^{+2}}\right) \nabla \times\left(\left(\tilde{\Psi}_{2}-\tilde{\Psi}_{1}\right) \mathbf{e}_{\varphi}\right)\right) \\
& =\frac{\rho_{0}^{+\gamma-1}}{(\gamma-1) u_{0}^{+}}\left(1+\frac{\gamma u_{0}^{+2}}{c_{0}^{+2}-u_{0}^{+2}}\right) \frac{\partial_{\theta}\left(\tilde{T}_{2}-\tilde{T}_{1}\right)}{r} \mathbf{e}_{\varphi}+A_{2}-A_{1}+B_{2}-B_{1} \\
& +\tilde{\boldsymbol{F}}_{12}\left(\tilde{\Psi}_{2} \mathbf{e}_{\varphi}, \tilde{A}_{2}, \tilde{T}_{2}\right)-\tilde{\boldsymbol{F}}_{11}\left(\tilde{\Psi}_{1} \mathbf{e}_{\varphi}, \tilde{A}_{1}, \tilde{T}_{1}\right)(=: \tilde{\boldsymbol{F}}) \text { in } \mathcal{N}_{r_{s}}^{+}, \\
& \left(\tilde{\Psi}_{2}-\tilde{\Psi}_{1}\right) \mathbf{e}_{\varphi} \\
& =\left\{\begin{array}{l}
\left(\frac{\Pi_{r_{s} f_{2}}^{*, r}\left(\Phi_{-}-\Phi_{0}^{-}\right)\left(\Pi_{r_{s} f_{2}}\right)-\Pi_{r_{s} f_{1}}^{*, r}\left(\Phi_{-}-\Phi_{0}^{-}\right)\left(\Pi_{r_{s} f_{1}}\right)}{r}\right) \mathbf{e}_{\varphi}\left(=: \tilde{\boldsymbol{h}}_{1}\right) \text { on } \Gamma_{r_{s}}, \\
\mathbf{0} \text { on } \Gamma_{w, r_{s}}^{+}, \\
\left(\frac { 1 } { r \operatorname { s i n } \theta } \int _ { 0 } ^ { \theta } \left(\mathfrak{f}_{0}\left(\tilde{T}_{2}, p_{e x}\right)-\mathfrak{f}_{0}\left(\tilde{T}_{1}, p_{e x}\right)-\frac{\rho_{0}^{+}\left((\gamma-1) u_{0}^{+2}+c_{0}^{+2}\right)}{\gamma(\gamma-1) u_{0}^{+} S_{0}^{+}}\left(\tilde{T}_{2}-\tilde{T}_{1}\right)\right.\right. \\
\left.\left.+\mathfrak{f}_{1}\left(\tilde{\Psi}_{2} \mathbf{e}_{\varphi}, \tilde{A}_{2}, \tilde{T}_{2}\right)-\mathfrak{f}_{1}\left(\tilde{\Psi}_{1} \mathbf{e}_{\varphi}, \tilde{A}_{1}, \tilde{T}_{1}\right)\right) r^{2} \sin \xi \mathrm{d} \xi\right) \mathbf{e}_{\varphi}\left(=: \tilde{\boldsymbol{h}}_{2}\right) \text { on } \Gamma_{e x},
\end{array}\right. \\
& \frac{1}{r \sin \theta} \int_{0}^{\theta}\left(\mathfrak{f}_{0}\left(\tilde{T}_{2}, p_{e x}\right)-\mathfrak{f}_{0}\left(\tilde{T}_{1}, p_{e x}\right)-\frac{\rho_{0}^{+}\left((\gamma-1) u_{0}^{+2}+c_{0}^{+2}\right)}{\gamma(\gamma-1) u_{0}^{+} S_{0}^{+}}\left(\tilde{T}_{2}-\tilde{T}_{1}\right)\right. \\
& \left.+\mathfrak{f}_{1}\left(\tilde{\Psi}_{2} \mathbf{e}_{\varphi}, \tilde{A}_{2}, \tilde{T}_{2}\right)-\mathfrak{f}_{1}\left(\tilde{\Psi}_{1} \mathbf{e}_{\varphi}, \tilde{A}_{1}, \tilde{T}_{1}\right)\right) r^{2} \sin \xi \mathrm{d} \xi=0,
\end{aligned}
$$

where

$$
\begin{aligned}
A_{i}=\nabla \times & \left(\frac{1}{\rho_{0}^{+}}\left(1+\frac{u_{0}^{+} \mathbf{e}_{r} \otimes u_{0}^{+} \mathbf{e}_{r}}{c_{0}^{+^{2}}-u_{0}^{+2}}\right) \nabla \times\left(\tilde{\Psi}_{i} \mathbf{e}_{\varphi}\right)\right) \\
& -M_{i} \nabla \times\left(\frac{1}{\rho_{0}^{+}}\left(1+\frac{u_{0}^{+} \mathbf{e}_{r} \otimes u_{0}^{+} \mathbf{e}_{r}}{c_{0}^{+2}-u_{0}^{+2}}\right)\left(\Pi_{r_{s} f_{i}}\right) N_{i} \nabla \times\left(\tilde{\Psi}_{i} \mathbf{e}_{\varphi}\right)\right)
\end{aligned}
$$


and

$$
\begin{aligned}
B_{i}= & -\frac{\rho_{0}^{+\gamma-1}}{(\gamma-1) u_{0}^{+}}\left(1+\frac{\gamma u_{0}^{+2}}{c_{0}^{+2}-u_{0}^{+2}}\right) \frac{\partial_{\theta} \tilde{T}_{i}}{r} \mathbf{e}_{\varphi} \\
& +\left(\frac{\rho_{0}^{+\gamma-1}}{(\gamma-1) u_{0}^{+}}\left(1+\frac{\gamma u_{0}^{+2}}{c_{0}^{+2}-u_{0}^{+2}}\right)\right)\left(\Pi_{r_{s} f_{i}}\right) \frac{\left(\partial_{\tilde{\theta}_{i}} \Pi_{f_{i} r_{s}}^{*, r}\right)\left(\Pi_{r_{s} f_{i}}\right) \partial_{r} \tilde{T}_{i}+\partial_{\theta} \tilde{T}_{i}}{\Pi_{r_{s} f_{i}}^{r}} \mathbf{e}_{\varphi}
\end{aligned}
$$

for $i=1,2$. We will estimate $\left\|\left(\tilde{\Psi}_{2}-\tilde{\Psi}_{1}\right) \mathbf{e}_{\varphi}\right\|_{1, \beta, \mathcal{N}_{r_{s}}^{+}}$using (4.47)-(4.49). For this, we estimate $\left\|\frac{\tilde{A}_{2}}{2 \pi r \sin \theta}-\frac{\tilde{A}_{1}}{2 \pi r \sin \theta}\right\|_{1,0, \mathcal{N}_{r_{s}}^{+}}$and $\left\|\tilde{T}_{2}-\tilde{T}_{1}\right\|_{0, \beta, \mathcal{N}_{r_{s}}^{+}}$.

Estimate $\left\|\frac{\tilde{A}_{2}}{2 \pi r \sin \theta}-\frac{\tilde{A}_{1}}{2 \pi r \sin \theta}\right\|_{0, \beta, \mathcal{N}_{r_{s}}^{+}}$and $\left\|\tilde{T}_{2}-\tilde{T}_{1}\right\|_{0, \beta, \mathcal{N}_{r_{s}}^{+}}$:

Since $A_{i}$ and $T_{i}$ for $i=1,2$ are solutions of $\left(\mathrm{B}^{\prime}\right)$ for $\left(\Psi, f(0), f_{s}\right)=\left(\Psi_{i}\right.$, $\left.f_{i}(0), f_{s, i}\right)$, by Lemma $15, A_{i}$ and $T_{i}$ are represented as

$$
A_{i}=2 \pi f_{i}\left(\mathcal{L}_{i}\right) \sin \mathcal{L}_{i} u_{-, \varphi}\left(f_{i}\left(\mathcal{L}_{i}\right), \mathcal{L}_{i}\right)
$$

and

$$
T_{i}=\left(g\left(\left(\frac{\mathbf{u}_{-} \cdot \mathbf{v}_{f_{i}}\left(\mathcal{L}_{i}\right)}{c_{-}}\right)^{2}\right) S_{-}\right)\left(f_{i}\left(\mathcal{L}_{i}\right), \mathcal{L}_{i}\right)-\left(g\left(M_{0}^{-2}\right)\right)\left(r_{s}\right) S_{i n}
$$

where $u_{-, \varphi}=\mathbf{u}_{-} \cdot \mathbf{e}_{\varphi}, \mathcal{L}_{i}$ for $i=1,2$ are $\mathcal{L}$ given in Lemma 15 for $V=$ $2 \pi r \sin \theta\left(\Phi_{0}^{+}+\Psi_{i}\right)$ and $f=f_{i}$, respectively, and $\boldsymbol{v}_{f_{i}}$ for $i=1,2$ are the unit normal vectors on $\Gamma_{f_{i}}$ pointing toward $\mathcal{N}_{f_{i}}^{+}$, respectively. Using these solution expressions, express $\left(\frac{\tilde{A}_{2}}{2 \pi r \sin \theta}-\frac{\tilde{A}_{1}}{2 \pi r \sin \theta}\right) \mathbf{e}_{\varphi}$ and $\tilde{T}_{2}-\tilde{T}_{1}$ as

$$
\left(\frac{2 \pi f_{2}\left(\tilde{\mathcal{L}}_{2}\right) \sin \tilde{\mathcal{L}}_{2} u_{\varphi,-}\left(f_{2}\left(\tilde{\mathcal{L}}_{2}\right), \tilde{\mathcal{L}}_{2}\right)}{2 \pi r \sin \theta}-\frac{2 \pi f_{1}\left(\tilde{\mathcal{L}}_{1}\right) \sin \tilde{\mathcal{L}}_{1} u_{\varphi,-}\left(f_{1}\left(\tilde{\mathcal{L}}_{1}\right), \tilde{\mathcal{L}}_{1}\right)}{2 \pi r \sin \theta}\right) \mathbf{e}_{\varphi}
$$

and

$$
\begin{aligned}
& \left(g\left(\left(\frac{\mathbf{u}_{-} \cdot \boldsymbol{v}_{f_{2}}\left(\tilde{\mathcal{L}}_{2}\right)}{c_{-}}\right)^{2}\right) S_{-}\right)\left(f_{2}\left(\tilde{\mathcal{L}}_{2}\right), \tilde{\mathcal{L}}_{2}\right) \\
& -\left(g\left(\left(\frac{\mathbf{u}_{-} \cdot \boldsymbol{v}_{f_{1}}\left(\tilde{\mathcal{L}}_{1}\right)}{c_{-}}\right)^{2}\right) S_{-}\right)\left(f_{1}\left(\tilde{\mathcal{L}}_{1}\right), \tilde{\mathcal{L}}_{1}\right),
\end{aligned}
$$

respectively, where $\tilde{\mathcal{L}}_{i}:=\mathcal{L}_{i}\left(\Pi_{r_{s}} f_{i}\right)$ for $i=1,2$. Using arguments similar to the ones used to estimate $\left\|\left(\frac{A_{2}}{2 \pi r \sin \theta}-\frac{\tilde{A}_{1}}{2 \pi r \sin \theta}\right) \mathbf{e}_{\varphi}\right\|_{1,0, \mathcal{N}_{f_{2}}^{+}}$and $\left\|T_{2}-\tilde{T}_{1}\right\|_{0, \beta, \mathcal{N}_{f_{2}}^{+}}$in the proof of the uniqueness part of Proposition 2, we estimate (4.50) and (4.51) in $C^{\beta}\left(\overline{\mathcal{N}_{r_{s}}^{+}}\right)$. Then we obtain 


$$
\begin{aligned}
& \left\|\left(\frac{\tilde{A}_{2}}{2 \pi r \sin \theta}-\frac{\tilde{A}_{1}}{2 \pi r \sin \theta}\right) \mathbf{e}_{\varphi}\right\|_{1,0, \mathcal{N}_{r_{s}}^{+}} \\
& \leq C \sigma\left(\left\|f_{2}-f_{1}\right\|_{1, \beta, \Lambda}+\left\|\left(\tilde{\Psi}_{2}-\tilde{\Psi}_{1}\right) \mathbf{e}_{\varphi}\right\|_{1, \beta, \mathcal{N}_{r}^{+}}\right)
\end{aligned}
$$

and

$$
\left\|\tilde{T}_{2}-\tilde{T}_{1}\right\|_{0, \beta, \mathcal{N}_{r s}^{+}} \leq C\left\|f_{2}-f_{1}\right\|_{1, \beta, \Lambda}+C \sigma\left\|\left(\tilde{\Psi}_{2}-\tilde{\Psi}_{1}\right) \mathbf{e}_{\varphi}\right\|_{1, \beta, \mathcal{N}_{r s}^{+}} .
$$

Using these estimates, we estimate $\left\|\left(\tilde{\Psi}_{2}-\tilde{\Psi}_{1}\right) \mathbf{e}_{\varphi}\right\|_{1, \beta, \mathcal{N}_{r_{s}}^{+}}$Substitute (4.51) into (4.49). And then using (4.52) and (4.53), estimate $f_{2}(0)-f_{1}(0)$ in the resultant equation (see Step 4 in the proof of the uniqueness part of Proposition 2). Then we obtain

$$
\begin{aligned}
& \left|f_{2}(0)-f_{1}(0)\right| \\
& \quad \leq C_{4} \sigma\left|f_{2}(0)-f_{1}(0)\right|+C|| f_{s, 2}^{\prime}-f_{s, 1}^{\prime}\left\|_{0, \beta,\left(0, \theta_{1}\right)}+C \sigma\right\|\left(\tilde{\Psi}_{2}-\tilde{\Psi}_{1}\right) \mathbf{e}_{\varphi} \|_{1, \beta, \mathcal{N}_{r s}^{+}} .
\end{aligned}
$$

Take $\sigma_{1}=\min \left(\sigma_{1}^{(1)}, \frac{1}{2 C_{4}}\right)\left(=: \sigma_{1}^{(2)}\right)$. Then we have

$$
\left|f_{2}(0)-f_{1}(0)\right| \leq C|| f_{s, 2}^{\prime}-f_{s, 1}^{\prime}\left\|_{0, \beta,\left(0, \theta_{1}\right)}+C \sigma\right\|\left(\tilde{\Psi}_{2}-\tilde{\Psi}_{1}\right) \mathbf{e}_{\varphi} \|_{1, \beta, \mathcal{N}_{r_{s}}^{+}} .
$$

Using (4.54), we obtain from (4.52) and (4.53) that

$$
\begin{aligned}
& \left\|\frac{\tilde{A}_{2}}{2 \pi r \sin \theta}-\frac{\tilde{A}_{1}}{2 \pi r \sin \theta}\right\|_{0, \beta, \mathcal{N}_{r_{s}}^{+}} \\
& \quad \leq C \sigma\left(\left\|f_{s, 2}^{\prime}-f_{s, 1}^{\prime}\right\|_{0, \beta,\left(0, \theta_{1}\right)}+\left\|\left(\tilde{\Psi}_{2}-\tilde{\Psi}_{1}\right) \mathbf{e}_{\varphi}\right\|_{0, \beta, \mathcal{N}_{r s}^{+}}\right)
\end{aligned}
$$

and

$$
\left\|\tilde{T}_{2}-\tilde{T}_{1}\right\|_{0, \beta, \mathcal{N}_{r_{s}}^{+}} \leq C|| f_{s, 2}^{\prime}-f_{s, 1}^{\prime}\left\|_{0, \beta,\left(0, \theta_{1}\right)}+C \sigma\right\|\left(\tilde{\Psi}_{2}-\tilde{\Psi}_{1}\right) \mathbf{e}_{\varphi} \|_{1, \beta, \mathcal{N}_{r_{s}}^{+}} .
$$

Using these two estimates, (4.54) and arguments similar to the ones in Step 5 in the proof of the uniqueness part of Proposition 2, we estimate $\left(\tilde{\Psi}_{2}-\tilde{\Psi}_{1}\right) \mathbf{e}_{\varphi}$ in (4.47), (4.48) in $C^{1, \beta}\left(\overline{\mathcal{N}_{r_{s}}^{+}}\right)$. Then we obtain

$$
\left\|\left(\tilde{\Psi}_{2}-\tilde{\Psi}_{1}\right) \mathbf{e}_{\varphi}\right\|_{1, \beta, \mathcal{N}_{r_{s}}^{+}} \leq C_{5} \sigma\left\|\left(\tilde{\Psi}_{2}-\tilde{\Psi}_{1}\right) \mathbf{e}_{\varphi}\right\|_{1, \beta, \mathcal{N}_{r_{s}}^{+}}+C\left\|f_{s, 2}^{\prime}-f_{s, 1}^{\prime}\right\|_{0, \beta,\left(0, \theta_{1}\right)} .
$$

Take $\sigma_{1}=\min \left(\sigma_{1}^{(2)}, \frac{1}{2 C_{5}}\right)\left(=: \sigma_{1}^{(3)}\right)$. Then we have

$$
\left\|\left(\tilde{\Psi}_{2}-\tilde{\Psi}_{1}\right) \mathbf{e}_{\varphi}\right\|_{1, \beta, \mathcal{N}_{r_{s}}^{+}} \leq C|| f_{s, 2}^{\prime}-f_{s, 1}^{\prime} \|_{0, \beta,\left(0, \theta_{1}\right)}
$$

Combining (4.54), (4.55), (4.56) and (4.57), we obtain (4.43).

2. Using the arguments in Step 1 in the proof of Lemma 17, we can see that the system (4.8)-(4.12) has a unique solution for given $\tilde{f}_{s}^{\prime}=f_{s, 2}^{\prime}-f_{s, 1}^{\prime} \in$ $C_{0}^{\beta}\left(\left[0, \theta_{1}\right]\right):=\left\{f \in C^{\beta}\left(\left[0, \theta_{1}\right]\right) \mid f^{\prime}(0)=f^{\prime}\left(\theta_{1}\right)=0\right\}:$

$$
f(0)^{\left(f_{s, 2}^{\prime}-f_{s, 1}^{\prime}\right)}=\frac{-\int_{0}^{\theta_{1}}\left(f_{s, 2}-f_{s, 1}\right) \sin \zeta d \zeta}{\int_{0}^{\theta_{1}} \sin \zeta d \zeta},
$$




$$
\begin{aligned}
& \tilde{A}^{\left(f_{s, 2}^{\prime}-f_{s, 1}^{\prime}\right)}=0 \\
& \tilde{T}^{\left(f_{s, 2}^{\prime}-f_{s, 1}^{\prime}\right)}=\left(g\left(M_{0}^{-2}\right)\right)^{\prime}\left(r_{s}\right) S_{i n}\left(\frac{-\int_{0}^{\theta_{1}}\left(f_{s, 2}-f_{s, 1}\right) \sin \zeta d \zeta}{\int_{0}^{\theta_{1}} \sin \zeta d \zeta}+f_{s, 2}-f_{s, 1}\right)
\end{aligned}
$$

and $\tilde{\Psi}^{\left(f_{s, 2}^{\prime}-f_{s, 1}^{\prime}\right)} \mathbf{e}_{\varphi}$, the unique $C_{\left(-1-\alpha, \Gamma_{w, r_{s}}^{+}\right)}^{2, \beta}\left(\mathcal{N}_{r_{s}}^{+}\right)$solution of (4.17), (4.18) for given $\tilde{f}_{s}^{\prime}=f_{s, 2}^{\prime}-f_{s, 1}^{\prime} \in C_{0}^{\beta}\left(\left[0, \theta_{1}\right]\right)$ (here we had $\tilde{\Psi}^{\left(f_{s, 2}^{\prime}-f_{s, 1}^{\prime}\right)} \mathbf{e}_{\varphi} \in C_{\left(-1-\alpha, \Gamma_{w, r_{s}}^{+}\right)}^{2, \beta}$ $\left(\mathcal{N}_{r_{s}}^{+}\right)$because $\left.f_{s, 2}^{\prime}-f_{s, 1}^{\prime} \in C_{0}^{\beta}\left(\left[0, \theta_{1}\right]\right)\right)$. Subtract (4.8)-(4.10) for given $\tilde{f}_{s}^{\prime}=$ $f_{s, 2}^{\prime}-f_{s, 1}^{\prime} \in C_{0}^{\beta}\left(\left[0, \theta_{1}\right]\right)$ from (4.47)-(4.49). Then we obtain

$$
\begin{aligned}
& \nabla \times\left(\frac{1}{\rho_{0}^{+}}\left(1+\frac{u_{0}^{+} \mathbf{e}_{r} \otimes u_{0}^{+} \mathbf{e}_{r}}{c_{0}^{+2}-u_{0}^{+2}}\right) \nabla \times\left(\left(\tilde{\Psi}_{2}-\tilde{\Psi}_{1}-\tilde{\Psi}^{\left(f_{s, 2}^{\prime}-f_{s, 1}^{\prime}\right)}\right) \mathbf{e}_{\varphi}\right)\right) \\
& =\frac{\rho_{0}^{+\gamma-1}}{(\gamma-1) u_{0}^{+}}\left(1+\frac{\gamma u_{0}^{+2}}{c_{0}^{+2}-u_{0}^{+2}}\right) \frac{\partial_{\theta}\left(\tilde{T}_{2}-\tilde{T}_{1}-\tilde{T}^{\left(f_{s, 2}^{\prime}-f_{s, 1}^{\prime}\right)}\right)}{r} \mathbf{e}_{\varphi} \\
& +A_{2}-A_{1}+B_{2}-B_{1}+\tilde{\boldsymbol{F}}_{12}\left(\tilde{\Psi}_{2} \mathbf{e}_{\varphi}, \tilde{A}_{2}, \tilde{T}_{2}\right) \\
& -\tilde{\boldsymbol{F}}_{11}\left(\tilde{\Psi}_{1} \mathbf{e}_{\varphi}, \tilde{A}_{1}, \tilde{T}_{1}\right) \text { in } \mathcal{N}_{r_{s}}^{+}, \\
& \left(\tilde{\Psi}_{2}-\tilde{\Psi}_{1}-\tilde{\Psi}^{\left(f_{s, 2}^{\prime}-f_{s, 1}^{\prime}\right)}\right) \mathbf{e}_{\varphi} \\
& =\left\{\begin{array}{l}
\left(\frac{\Pi_{r_{s} f_{2}}^{*, r}\left(\Phi_{-}-\Phi_{0}^{-}\right)\left(\Pi_{r_{s} f_{2}}\right)-\Pi_{r_{s} f_{1}}^{*, r}\left(\Phi_{-}-\Phi_{0}^{-}\right)\left(\Pi_{r_{s} f_{1}}\right)}{r}\right) \mathbf{e}_{\varphi} \text { on } \Gamma_{r_{s}}, \\
\mathbf{0} \text { on } \Gamma_{w, r_{s}}^{+}, \\
\left(\frac { 1 } { r \operatorname { s i n } \theta } \int _ { 0 } ^ { \theta } \left(\mathfrak{f}_{0}\left(\tilde{T}_{2}, p_{e x}\right)-\mathfrak{f}_{0}\left(\tilde{T}_{1}, p_{e x}\right)\right.\right. \\
\quad-\frac{\rho_{0}^{+}\left((\gamma-1) u_{0}^{+2}+c_{0}^{+2}\right)}{\gamma(\gamma-1) u_{0}^{+} S_{0}^{+}}\left(\tilde{T}_{2}-\tilde{T}_{1}-\tilde{T}^{\left(f_{s, 2}^{\prime}-f_{s, 1}^{\prime}\right)}\right) \\
\left.\left.+\tilde{f}_{1}\left(\tilde{\Psi}_{2} \mathbf{e}_{\varphi}, \tilde{A}_{2}, \tilde{T}_{2}\right)-\mathfrak{f}_{1}\left(\tilde{\Psi}_{1} \mathbf{e}_{\varphi}, \tilde{A}_{1}, \tilde{T}_{1}\right)\right) r^{2} \sin \xi \mathrm{d} \xi\right) \mathbf{e}_{\varphi} \text { on } \Gamma_{e x},
\end{array}\right. \\
& \frac{1}{r \sin \theta} \int_{0}^{\theta}\left(\mathfrak{f}_{0}\left(\tilde{T}_{2}, p_{e x}\right)-\mathfrak{f}_{0}\left(\tilde{T}_{1}, p_{e x}\right)\right. \\
& -\frac{\rho_{0}^{+}\left((\gamma-1) u_{0}^{+2}+c_{0}^{+2}\right)}{\gamma(\gamma-1) u_{0}^{+} S_{0}^{+}}\left(\tilde{T}_{2}-\tilde{T}_{1}-\tilde{T}^{\left(f_{s, 2}^{\prime}-f_{s, 1}^{\prime}\right)}\right) \\
& \left.+\mathfrak{f}_{1}\left(\tilde{\Psi}_{2} \mathbf{e}_{\varphi}, \tilde{A}_{2}, \tilde{T}_{2}\right)-\mathfrak{f}_{1}\left(\tilde{\Psi}_{1} \mathbf{e}_{\varphi}, \tilde{A}_{1}, \tilde{T}_{1}\right)\right) r^{2} \sin \xi \mathrm{d} \xi=0 .
\end{aligned}
$$

Estimate $\left\|\tilde{\Psi}_{2}-\tilde{\Psi}_{1}-\tilde{\Psi}^{\left(f_{s, 2}^{\prime}-f_{s, 1}^{\prime}\right)}\right\|_{1, \beta, \mathcal{N}_{r_{s}}^{+}} \operatorname{using}(4.61)-(4.63)$.

Write $\tilde{T}^{\left(f_{s, 2}^{\prime}-f_{s, 1}^{\prime}\right)}$ as

$$
\left(g\left(M_{0}^{-2}\right)\right)^{\prime}\left(r_{s}\right) S_{i n}\left(f(0)^{\left(f_{s, 2}^{\prime}-f_{s, 1}^{\prime}\right)}+f_{s, 2}-f_{s, 1}\right)
$$


Substitute this $\tilde{T}^{\left(f_{s, 2}^{\prime}-f_{s, 1}^{\prime}\right)}$ and (4.51) into the places of $\tilde{T}^{\left(f_{s, 2}^{\prime}-f_{s, 1}^{\prime}\right)}$ and $\tilde{T}_{2}-\tilde{T}_{1}$ in (4.63), respectively. And then using (4.43), estimate $f_{2}(0)-f_{1}(0)-f(0)^{\left(f_{s, 2}^{\prime}-f_{s, 1}^{\prime}\right)}$ in the resultant equation. Then we obtain

$$
\left|f_{2}(0)-f_{1}(0)-f(0)^{\left(f_{s, 2}^{\prime}-f_{s, 1}^{\prime}\right)}\right| \leq C \sigma\left\|f_{s, 2}^{\prime}-f_{s, 1}^{\prime}\right\|_{0, \beta,\left(0, \theta_{1}\right)} .
$$

Using this estimate and (4.43), we estimate $\tilde{T}_{2}-\tilde{T}_{1}-\tilde{T}^{\left(f_{s, 2}^{\prime}-f_{s, 1}^{\prime}\right)}$ given by (4.51) and (4.64) in $C^{\beta}\left(\overline{\mathcal{N}_{r_{s}}^{+}}\right)$. Then we obtain

$$
\left\|\tilde{T}_{2}-\tilde{T}_{1}-\tilde{T}^{\left(f_{s, 2}^{\prime}-f_{s, 1}^{\prime}\right)}\right\|_{0, \beta, \mathcal{N}_{r_{s}}^{+}} \leq C \sigma\left\|f_{s, 2}^{\prime}-f_{s, 1}^{\prime}\right\|_{0, \beta,\left(0, \theta_{1}\right)} .
$$

Using arguments similar to the ones in Step 5 in the proof of the uniqueness part of Proposition 2 with (4.43) and (4.65), we estimate $\tilde{\Psi}_{2}-\tilde{\Psi}_{1}-\tilde{\Psi}^{\left(f_{s, 2}^{\prime}-f_{s, 1}^{\prime}\right)}$ in (4.61), (4.62) in $C^{1, \beta}\left(\overline{\mathcal{N}_{r_{s}}^{+}}\right)$. Then we have

$$
\left\|\tilde{\Psi}_{2}-\tilde{\Psi}_{1}-\tilde{\Psi}^{\left(f_{s, 2}^{\prime}-f_{s, 1}^{\prime}\right)}\right\|_{1, \beta, \mathcal{N}_{r_{s}}^{+}} \leq C \sigma\left\|f_{s, 2}^{\prime}-f_{s, 1}^{\prime}\right\|_{0, \beta,\left(0, \theta_{1}\right)} .
$$

3. By the assumption, $\left(f_{i}, \Phi_{i} \mathbf{e}_{\varphi}, L_{i}, S_{i}\right)$ for $i=1,2$ satisfy (2.47). This implies that $\mathcal{A}\left(\rho_{-}, \mathbf{u}_{-}, p_{-}, p_{e x}, f_{s, i}^{\prime}\right)=0$ for $i=1,2$ where $\mathcal{A}$ is a map defined in (4.1). Subtract these two equations. Then we have

$$
\mathcal{A}\left(\rho_{-}, \mathbf{u}_{-}, p_{-}, p_{\text {ex }}, f_{s, 2}^{\prime}\right)-\mathcal{A}\left(\rho_{-}, \mathbf{u}_{-}, p_{-}, p_{\text {ex }}, f_{s, 1}^{\prime}\right)=0 .
$$

Write this as

$$
0=D_{f_{s}^{\prime}} \mathcal{A}\left(\zeta_{0}\right)\left(f_{s, 2}^{\prime}-f_{s, 1}^{\prime}\right)+R
$$

where

$$
\begin{aligned}
R= & \mathcal{A}\left(\rho_{-}, \mathbf{u}_{-}, p_{-}, p_{e x}, f_{s, 2}^{\prime}\right)-\mathcal{A}\left(\rho_{-}, \mathbf{u}_{-}, p_{-}, p_{e x}, f_{s, 1}^{\prime}\right) \\
& -D_{f_{s}^{\prime}} \mathcal{A}\left(\zeta_{0}\right)\left(f_{s, 2}^{\prime}-f_{s, 1}^{\prime}\right),
\end{aligned}
$$

and $D_{f_{s}^{\prime}} \mathcal{A}\left(\zeta_{0}\right)$ is a map given in (4.2) as a map from $C_{0}^{\beta}\left(\left[0, \theta_{1}\right]\right)$ to $C_{0}^{\beta}\left(\left[0, \theta_{1}\right]\right)$ (here $D_{f_{s}^{\prime}} \mathcal{A}\left(\zeta_{0}\right)$ takes $C_{0}^{\beta}\left(\left[0, \theta_{1}\right]\right)$ functions and $\tilde{\Psi}^{\left(f_{s, 2}^{\prime}-f_{s, 1}^{\prime}\right)} \mathbf{e}_{\varphi}$ in $D_{f_{s}^{\prime}} \mathcal{A}\left(\zeta_{0}\right)\left(f_{s, 2}^{\prime}-f_{s, 1}^{\prime}\right)$ is the $C_{\left(-1-\alpha, \Gamma_{w, r_{s}}^{+}\right)}^{2, \beta}\left(\mathcal{N}_{r_{s}}^{+}\right)$solution of (4.17), (4.18) for given $\tilde{f}_{s}^{\prime}=f_{s, 2}^{\prime}-f_{s, 1}^{\prime} \in$ $\left.C_{0}^{\beta}\left(\left[0, \theta_{1}\right]\right)\right)$.

Using (4.43) and (4.66), we can obtain

$$
\|R\|_{0, \beta,\left(0, \theta_{1}\right)} \leq C \sigma\left\|f_{s, 2}^{\prime}-f_{s, 1}^{\prime}\right\|_{0, \beta,\left(0, \theta_{1}\right)} .
$$

In the way that we proved the invertiblity of $D_{f_{s}^{\prime}} \mathcal{A}\left(\zeta_{0}\right)$ as a map from $C_{\left(-\alpha,\left\{\theta=\theta_{1}\right\}\right)}^{1, \alpha}$ $((0, \theta))$ to $C_{\left(-\alpha,\left\{\theta=\theta_{1}\right\}\right)}^{1, \alpha}((0, \theta))$ in the the proof of Lemma 19, we can prove that $D_{f_{s}^{\prime}} \mathcal{A}\left(\zeta_{0}\right)$ is an invertible map as a map from $C_{0}^{\beta}\left(\left[0, \theta_{1}\right]\right)$ to $C_{0}^{\beta}\left(\left[0, \theta_{1}\right]\right)$. Using this fact and (4.68), we obtain, from (4.67),

$$
\left\|f_{s, 2}^{\prime}-f_{s, 1}^{\prime}\right\|_{0, \beta,\left(0, \theta_{1}\right)} \leq C_{6} \sigma\left\|f_{s, 2}^{\prime}-f_{s, 1}^{\prime}\right\|_{0, \beta,\left(0, \theta_{1}\right)} .
$$

Take $\sigma_{1}=\min \left(\sigma_{1}^{(3)}, \frac{1}{2 C_{6}}\right)\left(=: \underline{\sigma}_{1}\right)$. Then we have $f_{s, 2}^{\prime}=f_{s, 1}^{\prime}$. One can see that $\underline{\sigma}_{1}$ depends on the data. This finishes the proof. 
Acknowledgements. The author would like to thank Myoungjean Bae for her advice in doing this work. The research of Yong Park was supported in part by Samsung Science and Technology Foundation under Project Number SSTF-BA1502-02.

Open Access This article is licensed under a Creative Commons Attribution 4.0 International License, which permits use, sharing, adaptation, distribution and reproduction in any medium or format, as long as you give appropriate credit to the original author(s) and the source, provide a link to the Creative Commons licence, and indicate if changes were made. The images or other third party material in this article are included in the article's Creative Commons licence, unless indicated otherwise in a credit line to the material. If material is not included in the article's Creative Commons licence and your intended use is not permitted by statutory regulation or exceeds the permitted use, you will need to obtain permission directly from the copyright holder. To view a copy of this licence, visit http://creativecommons.org/ licenses/by/4.0/.

Publisher's Note Springer Nature remains neutral with regard to jurisdictional claims in published maps and institutional affiliations.

\section{Appendix}

In this section, we present some computations done by using the tensor notation given in $\S 3.2$, and make up some fact needed for the well-definedness of $V$ defined in (2.23). We explain how we transformed an elliptic system on the Cartesian coordinate system into a system on the spherical coordinate system in the proof of Lemma 10 and Lemma 11, and show that (3.35) is equivalent to (3.28) and that $V$ defined in (2.23) is independent of the choice of $S_{\mathrm{x}}$.

Let $\left(q_{1}, q_{2}, q_{3}\right)$ be an orthogonal coordinate system in $\mathbb{R}^{3}$. The unit vectors in this coordinate system in the direction of $q_{i}$ for $i=1,2,3$ are given as $\frac{1}{h_{i}} \frac{\partial \boldsymbol{x}}{\partial q_{i}}\left(=: \mathbf{e}_{q_{i}}\right)$ for $i=1,2,3$ where $\boldsymbol{x}=x \mathbf{e}_{1}+y \mathbf{e}_{2}+z \mathbf{e}_{3}$ and $h_{i}:=\left|\frac{\partial \boldsymbol{x}}{\partial q_{i}}\right|$. By $\nabla=\sum_{i=1}^{3} \frac{\mathbf{e}_{q_{i}}}{h_{i}} \partial_{q_{i}}, \nabla \boldsymbol{U}$ where $\boldsymbol{U}: \mathbb{R}^{3} \rightarrow \mathbb{R}^{3}$ can be written as

$$
\nabla \boldsymbol{U}=\sum_{i=1}^{3} \frac{\partial_{q_{i}} \boldsymbol{U}}{h_{i}} \otimes \mathbf{e}_{q_{i}}
$$

Here, $\boldsymbol{a} \otimes \boldsymbol{b}$ for $\boldsymbol{a}, \boldsymbol{b} \in \mathbb{R}^{3}$ denotes $\boldsymbol{a} \boldsymbol{b}^{T}$. By (3.34), the multiplication of the tensor notation defined in $\S 3.2$ is defined by

$$
(a \otimes b \otimes c \otimes d)(e \otimes f \otimes g \otimes h)=(d \cdot e)(c \cdot f)(a \otimes b \otimes g \otimes h),
$$

where $\boldsymbol{a}, \boldsymbol{b}, \boldsymbol{c}, \boldsymbol{d}, \boldsymbol{e}, \boldsymbol{f}, \boldsymbol{g}, \boldsymbol{h} \in \mathbb{R}^{3}$. By (5.1) and (5.2), $M$ in

$$
\nabla \boldsymbol{U}=M \nabla_{\left(q_{1}, q_{2}, q_{3}\right)} \boldsymbol{U}
$$

where $\nabla_{\left(q_{1}, q_{2}, q_{3}\right)} \boldsymbol{U}=\sum_{i=1}^{3} \partial_{q_{i}} \boldsymbol{U} \otimes \mathbf{e}_{i}$ and $M=\frac{\partial\left(q_{1}, q_{2}, q_{3}\right)}{\partial(x, y, z)}$, can be expressed as

$$
M=\sum_{i=1}^{3} \frac{1}{h_{i}} \mathcal{I} \otimes \mathbf{e}_{q_{i}} \otimes \mathbf{e}_{i} \otimes \mathcal{I} .
$$

Here, $\mathcal{I} \otimes \boldsymbol{a} \otimes \boldsymbol{b} \otimes \mathcal{I}$ for $\boldsymbol{a}, \boldsymbol{b} \in R^{3}$ is a linear map from $\mathbb{R}^{3 \times 3}$ to $\mathbb{R}^{3 \times 3}$ defined by

$$
\mathcal{I} \otimes \boldsymbol{a} \otimes \boldsymbol{b} \otimes \mathcal{I}:=\sum_{i=1}^{3} \mathbf{e}_{i} \otimes \boldsymbol{a} \otimes \boldsymbol{b} \otimes \mathbf{e}_{i} .
$$


(Note that $\mathcal{I} \otimes \boldsymbol{a} \otimes \boldsymbol{b} \otimes \mathcal{I}$ satisfies

$$
(\mathcal{I} \otimes \boldsymbol{a} \otimes \boldsymbol{b} \otimes \mathcal{I})(\boldsymbol{c} \otimes \boldsymbol{d})=(\boldsymbol{b} \cdot \boldsymbol{d}) \boldsymbol{c} \otimes \boldsymbol{a}
$$

for $\boldsymbol{c}, \boldsymbol{d} \in \mathbb{R}^{3}$.) Using these facts, we can transform an elliptic system on the Cartesian coordinate system into a system on another coordinate system. In the standard way, we transform $\operatorname{div}(\boldsymbol{B} \nabla \boldsymbol{U})=0$ on the Cartesian coordinate system where $\boldsymbol{B}$ is a linear map from $\mathbb{R}^{3 \times 3}$ to $\mathbb{R}^{3 \times 3}$ into an equation on $\left(q_{1}, q_{2}, q_{3}\right)$-coordinate system. Then we obtain

$$
\operatorname{div}_{\left(q_{1}, q_{2}, q_{3}\right)}\left(\frac{1}{\operatorname{det} M} M^{T} \boldsymbol{B} \circ \chi^{-1} M \nabla_{\left(q_{1}, q_{2}, q_{3}\right)}\left(\boldsymbol{U} \circ \chi^{-1}\right)\right)=0,
$$

where $\operatorname{div}_{\left(q_{1}, q_{2}, q_{3}\right)}:=\nabla_{\left(q_{1}, q_{2}, q_{3}\right)}, \nabla_{\left(q_{1}, q_{2}, q_{3}\right)}$ and $M$ are as above, $\chi$ is the map from $(x, y, z)$ to $\left(q_{1}, q_{2}, q_{3}\right)$ and det $M=\frac{1}{h_{1} h_{2} h_{3}}$. Using (5.2) and (5.3), we compute $M^{T} \boldsymbol{B} \circ$ $\chi^{-1} M$. Then we obtain the explicit form of (5.6). In this way, we can obtain the explicit form of the spherical coordinate representation of (3.57).

Next, we show that (3.35) is equivalent to (3.28).

By (5.1),

$$
\nabla\left(\Psi \mathbf{e}_{\varphi}\right)=\partial_{r} \Psi \mathbf{e}_{\varphi} \otimes \mathbf{e}_{r}+\frac{\partial_{\theta} \Psi}{r} \mathbf{e}_{\varphi} \otimes \mathbf{e}_{\theta}-\frac{\Psi}{r} \mathbf{e}_{r} \otimes \mathbf{e}_{\varphi}-\frac{\cos \theta}{r \sin \theta} \Psi \mathbf{e}_{\theta} \otimes \mathbf{e}_{\varphi}
$$

Using (5.5) and (5.7), we have

$$
\begin{aligned}
& \frac{c_{0}^{+2}}{\rho_{0}^{+}\left(c_{0}^{+^{2}}-u_{0}^{+^{2}}\right)}\left(\boldsymbol{I}-\frac{u_{0}^{+2}}{c_{0}^{+^{2}}}\left(\mathcal{I} \otimes \mathbf{e}_{r} \otimes \mathbf{e}_{r} \otimes \mathcal{I}\right)\right) \nabla\left(\Psi \mathbf{e}_{\varphi}\right) \\
& =\frac{c_{0}^{+2}}{\rho_{0}^{+}\left(c_{0}^{+2}-u_{0}^{+2}\right)} \nabla\left(\Psi \mathbf{e}_{\varphi}\right)-\frac{u_{0}^{+^{2}}}{\rho_{0}^{+}\left(c_{0}^{+^{2}}-u_{0}^{+2}\right)} \partial_{r} \Psi \mathbf{e}_{\varphi} \otimes \mathbf{e}_{r} .
\end{aligned}
$$

Using this relation and the relation $\operatorname{div}(\boldsymbol{a} \otimes \boldsymbol{b})=\nabla \boldsymbol{a} \boldsymbol{b}+\boldsymbol{a} \operatorname{div} \boldsymbol{b}$, we compute the left hand side of (3.35). Then we get

$$
\begin{gathered}
a(r) \Delta\left(\Psi \mathbf{e}_{\varphi}\right)+\nabla\left(\Psi \mathbf{e}_{\varphi}\right) \nabla a(r)-\nabla \mathbf{e}_{\varphi} b(r) \partial_{r} \Psi \mathbf{e}_{r} \\
-\mathbf{e}_{\varphi} \operatorname{div}\left(b(r) \partial_{r} \Psi \mathbf{e}_{r}\right)-\frac{\partial_{r} \rho_{0}^{+}}{\rho_{0}^{+2} r} \Psi \mathbf{e}_{\varphi}
\end{gathered}
$$

where $a(r):=\frac{c_{0}^{+2}}{\rho_{0}^{+}\left(c_{0}^{+2}-u_{0}^{+2}\right)}$ and $b(r):=\frac{u_{0}^{+2}}{\rho_{0}^{+}\left(c_{0}^{+2}-u_{0}^{+2}\right)}$. By direct computation, one can check that $\nabla \mathbf{e}_{\varphi} b(r) \partial_{r} \Psi \mathbf{e}_{r}=0$. Using this fact and (5.7), we compute (5.8). Then we have

$$
\begin{aligned}
& \left(a(r)\left(\Delta \Psi-\frac{\Psi}{r^{2} \sin ^{2} \theta}\right)+a^{\prime}(r) \partial_{r} \Psi-b(r)\left(\frac{1}{r^{2}} \partial_{r}\left(r^{2} \partial_{r} \Psi\right)\right)-b^{\prime}(r) \partial_{r} \Psi\right) \mathbf{e}_{\varphi} \\
& -\frac{\partial_{r} \rho_{0}^{+}}{\rho_{0}^{+2} r} \Psi \mathbf{e}_{\varphi} .
\end{aligned}
$$

By $a-b=\frac{1}{\rho_{0}^{+}},(a-b)^{\prime}=-\frac{\partial_{r} \rho_{0}^{+}}{\rho_{0}^{+2}}$. Substitute this relation into (5.9) and then express the resultant equation in the spherical coordinate system. Then we obtain 


$$
\begin{aligned}
& \left(\frac{1}{\rho_{0}^{+} r^{2}} \partial_{r}\left(r^{2} \partial_{r} \Psi\right)-\frac{\partial_{r} \rho_{0}^{+}}{\rho_{0}^{+2} r} \partial_{r}(r \Psi)\right. \\
& \left.+\frac{c_{0}^{+2}}{\rho_{0}^{+}\left(c_{0}^{+2}-u_{0}^{+2}\right) r^{2}}\left(\frac{1}{\sin \theta} \partial_{\theta}\left(\sin \theta \partial_{\theta} \Psi\right)-\frac{\Psi}{\sin ^{2} \theta}\right)\right) \mathbf{e}_{\varphi} .
\end{aligned}
$$

This after multiplied by $(-1)$ is equal to the left hand side of (3.30). By this fact, (3.35) is equivalent to (3.28).

Finally, for the well-definedness of $V$ defined in (2.23), we show that $V$ defined in (2.23) is independent of the choice of $S_{\mathrm{X}}$.

Lemma 20. Let $\Omega$ be an open simply connected axisymmetric set in $\mathbb{R}^{3}$. Let $S_{\mathrm{x}}^{1}$ and $S_{\mathrm{x}}^{2}$ be two different simply connected open bounded $C^{1}$ surfaces in $\Omega$ having the same boundary. Then

$$
\int_{S_{\mathrm{x}}^{1}} \rho \mathbf{u} \cdot v_{1} d S=\int_{S_{\mathrm{x}}^{2}} \rho \mathbf{u} \cdot \boldsymbol{v}_{2} d S,
$$

where $\boldsymbol{v}_{i}$ for $i=1,2$ are the unit normal vector on $S_{\mathrm{x}}^{i}$ pointing outward direction with respect to the cone-like domain made by connecting $\partial S_{\mathrm{x}}^{i}$ and the origin by straight lines, respectively.

Proof. The result can be obtained by using the divergence theorem.

Suppose that there exists a simply connected open bounded $C^{1}$ surface in $\Omega$ whose boundary is equal to the boundary of $S_{\mathrm{x}}^{1}$ or $S_{\mathrm{x}}^{2}$, and not intersecting with $S_{\mathrm{x}}^{1}$ or $S_{\mathrm{x}}^{2}$ except the boundary of the surface. Let us denote this surface by $S^{*}$. Then by the first equation of (2.1) and the divergence theorem, we have that

$$
\int_{S_{\mathrm{x}}^{i}} \rho \mathbf{u} \cdot \boldsymbol{v}_{i} d S=\int_{S^{*}} \rho \mathbf{u} \cdot \boldsymbol{v}^{*} d S
$$

for $i=1,2$, where $v^{*}$ is the unit normal vector on $S^{*}$ pointing outward direction with respect to the cone-like domain made by connecting $\partial S^{*}$ and the origin by straight lines. From this, we obtain (5.10). The existence of $S^{*}$ can be obtained by using the fact that $\Omega$ is open. This finishes the proof.

\section{References}

1. Ambrosio, L., Carlotto, A., Massaccesi, A.: Lectures on elliptic partial differential equations, Appunti. Scuola Normale Superiore di Pisa (Nuova Serie) [Lecture Notes. Scuola Normale Superiore di Pisa (New Series)]], vol. 18. Edizioni della Normale, Pisa 2018. https://doi.org/10.1007/978-88-7642-443-4

2. Bae, M., Duan, B., XIE, C.: Subsonic flow for the multidimensional Euler-Poisson system. Arch. Ration. Mech. Anal. 220(1), 155-191, 2016. https://doi.org/10.1007/s00205015-0930-6

3. Bae, M., Feldman, M.: Transonic shocks in multidimensional divergent nozzles. Arch. Ration. Mech. Anal. 201(3), 777-840, 2011. https://doi.org/10.1007/s00205-011-04240

4. Bae, M., Weng, S.: 3-D axisymmetric subsonic flows with nonzero swirl for the compressible Euler-Poisson system. Ann. Inst. H. Poincaré Anal. Non Linéaire 35(1), 161186, 2018. https://doi.org/10.1016/j.anihpc.2017.03.004 
5. Chen, G.Q., Chen, J., Feldman, M.: Transonic shocks and free boundary problems for the full Euler equations in infinite nozzles. J. Math. Pures Appl. (9) 88(2), 191-218, 2007. https://doi.org/10.1016/j.matpur.2007.04.008

6. Chen, G.Q., Chen, J., Song, K.: Transonic nozzle flows and free boundary problems for the full Euler equations. J. Differ. Equ. 229(1), 92-120, 2006. https://doi.org/10. 1016/j.jde.2006.04.015

7. Chen, G.Q., Feldman, M.: Multidimensional transonic shocks and free boundary problems for nonlinear equations of mixed type. J. Am. Math. Soc. 16(3), 461-494, 2003. https://doi.org/10.1090/S0894-0347-03-00422-3

8. Chen, G.Q., Feldman, M.: Steady transonic shocks and free boundary problems for the Euler equations in infinite cylinders. Commun. Pure Appl. Math. 57(3), 310-356, 2004. https://doi.org/10.1002/cpa.3042

9. Chen, G.Q., Feldman, M.: Existence and stability of multidimensional transonic flows through an infinite nozzle of arbitrary cross-sections. Arch. Ration. Mech. Anal. 184(2), 185-242, 2007. https://doi.org/10.1007/s00205-006-0025-5

10. Chen, G.Q.G., YUAN, H.: Local uniqueness of steady spherical transonic shock-fronts for the three-dimensional full Euler equations. Commun. Pure Appl. Anal. 12(6), 25152542, 2013. https://doi.org/10.3934/cpaa.2013.12.2515

11. Chen, S.: Stability of transonic shock fronts in two-dimensional Euler systems. Trans. Am. Math. Soc. 357(1), 287-308, 2005. https://doi.org/10.1090/S0002-994704-03698-0

12. Chen, S.: Transonic shocks in 3-D compressible flow passing a duct with a general section for Euler systems. Trans. Am. Math. Soc. 360(10), 5265-5289, 2008. https:// doi.org/10.1090/S0002-9947-08-04493-0

13. Chen, S., Yuan, H.: Transonic shocks in compressible flow passing a duct for threedimensional Euler systems. Arch. Ration. Mech. Anal. 187(3), 523-556, 2008. https:// doi.org/10.1007/s00205-007-0079-z

14. Courant, R., Friedrichs, K.O.: Supersonic flow and shock waves. SpringerVerlag, New York-Heidelberg 1976. https://mathscinet.ams.org/mathscinet-getitem? $\mathrm{mr}=0421279$. Reprinting of the 1948 original, Applied Mathematical Sciences, Vol. 21

15. Giaquinta, M.: Multiple integrals in the calculus of variations and nonlinear elliptic systems, Annals of Mathematics Studies, vol. 105. Princeton University Press, Princeton, NJ 1983. https://mathscinet.ams.org/mathscinet-getitem?mr=717034

16. Giaquinta, M., Martinazzi, L.: An introduction to the regularity theory for elliptic systems, harmonic maps and minimal graphs, Appunti. Scuola Normale Superiore di Pisa (Nuova Serie) [Lecture Notes. Scuola Normale Superiore di Pisa (New Series)], vol. 11, second edn. Edizioni della Normale, Pisa 2012. https://doi.org/10.1007/97888-7642-443-4. https://mathscinet.ams.org/mathscinet-getitem?mr=3099262

17. Han, Q., Lin, F.: Elliptic partial differential equations, Courant Lecture Notes in Mathematics, vol. 1, second edn. Courant Institute of Mathematical Sciences, New York; American Mathematical Society, Providence, RI 2011. https://mathscinet.ams. org/mathscinet-getitem?mr=2777537

18. Li, J., XIN, Z., YIN, H.: A free boundary value problem for the full Euler system and 2-D transonic shock in a large variable nozzle. Math. Res. Lett. 16(5), 777-796, 2009. https://doi.org/10.4310/MRL.2009.v16.n5.a3

19. Li, J., XIN, Z., Yin, H.: On transonic shocks in a nozzle with variable end pressures. Commun. Math. Phys. 291(1), 111-150, 2009. https://doi.org/10.1007/s00220-009-0870-9

20. LI, J., XIN, Z., YIN, H.: On transonic shocks in a conic divergent nozzle with axisymmetric exit pressures. J. Differ. Equ. 248(3), 423-469, 2010. https://doi.org/10. 1016/j.jde.2009.09.017

21. LI, J., XIN, Z., YIN, H.: Transonic shocks for the full compressible Euler system in a general two-dimensional de Laval nozzle. Arch. Ration. Mech. Anal. 207(2), 533-581, 2013. https://doi.org/10.1007/s00205-012-0580-x 
22. LiU, J.G., WANG, W.C.: Characterization and regularity for axisymmetric solenoidal vector fields with application to Navier-Stokes equation. SIAM J. Math. Anal. 41(5), 1825-1850, 2009. https://doi.org/10.1137/080739744

23. Liu, L., Xu, G., YuAN, H.: Stability of spherically symmetric subsonic flows and transonic shocks under multidimensional perturbations. Adv. Math. 291, 696-757, 2016. https://doi.org/10.1016/j.aim.2016.01.002

24. LiU, L., YuAn, H.: Stability of cylindrical transonic shocks for the two-dimensional steady compressible Euler system. J. Hyperbolic Differ. Equ. 5(2), 347-379, 2008. https://doi.org/10.1142/S0219891608001519

25. Xin, Z., YAN, W., Yin, H.: Transonic shock problem for the Euler system in a nozzle. Arch. Ration. Mech. Anal. 194(1), 1-47, 2009. https://doi.org/10.1007/s00205-0090251-8

26. XIN, Z., YIn, H.: Transonic shock in a nozzle. I. Two-dimensional case. Commun. Pure Appl. Math. 58(8), 999-1050, 2005. https://doi.org/10.1002/cpa.20025

27. XIN, Z., YIn, H.: Three-dimensional transonic shocks in a nozzle. Pac. J. Math. 236(1), 139-193, 2008. https://doi.org/10.2140/pjm.2008.236.139

28. XIN, Z., YIN, H.: The transonic shock in a nozzle, 2-D and 3-D complete Euler systems. J. Differ. Equ. 245(4), 1014-1085, 2008. https://doi.org/10.1016/j.jde.2008.04.010

29. YuAn, H.: On transonic shocks in two-dimensional variable-area ducts for steady Euler system. SIAM J. Math. Anal. 38(4), 1343-1370, 2006. https://doi.org/10.1137/ 050642447

30. Yuan, H.: Transonic shocks for steady Euler flows with cylindrical symmetry. Nonlinear Anal. 66(8), 1853-1878, 2007. https://doi.org/10.1016/j.na.2006.02.045

31. YUAN, H.: A remark on determination of transonic shocks in divergent nozzles for steady compressible Euler flows. Nonlinear Anal. Real World Appl. 9(2), 316-325, 2008. https://doi.org/10.1016/j.nonrwa.2006.10.006

32. Yuan, H., Zhao, Q.: Stabilization effect of frictions for transonic shocks in steady compressible euler flows passing three-dimensional ducts 2018

\author{
Y. Park \\ POSTECH, \\ San 31, Hyojadong, Namgu, \\ Pohang \\ Gyungbuk \\ 37673 Republic of Korea. \\ e-mail: pipablue@postech.ac.kr
}

(Received August 16, 2019 / Accepted February 2, 2021)

Published online February 21, 2021

(C) The Author(s) (2021) 